\title{
$(4$
}

UNIVERSIDAD PERUANA DE CIENCIAS APLICADAS

FACULTAD DE INGENIERÍA

PROGRAMA ACADÉMICO DE INGENIERÍA DE SISTEMAS

\section{PROPUESTA DE UNA ARQUITECTURA \\ EMPRESARIAL PARA UNA CONSULTORA DE SOFTWARE}

\author{
TESIS \\ Para optar por el título profesional de Ingeniero de Sistemas \\ AUTOR \\ ROJAS ARROYO, RAAL PABLO (0000-0002-6756-3188) \\ DÍAZ CHÁVEZ, PATRICIA MILAGROS (0000-0003-1768-3234)
}

ASESOR DE TESIS

ARMAS AGUIRRE, JIMMY ALEXANDER (0000-0002-1176-8969)

Lima, 23 de Junio de 2018 
(1)

DEDICATORIA 


\section{(}

El presente proyecto está dedicado a nuestras familias quienes fueron testigo de todo el esfuerzo y perseverancia que tuvimos para cumplir esta meta en nuestra formación profesional. 


\section{(1)}

\section{AGRADECIMIENTOS}

Agradecemos a nuestras familias por todo su apoyo brindado a lo largo de nuestra etapa universitaria. De igual manera a nuestros profesores, por toda su orientación, valores, herramientas y primordialmente por el conocimiento inculcado durante este largo camino, el cual nos ha convertido en mejores personas y profesionales. 


\section{(1)}

\section{RESUMEN}

En el presente proyecto profesional se desarrolla la propuesta de implementación de una Arquitectura Empresarial para una consultora de software, para la cual se ha tenido que realizar el análisis de la situación actual (AS IS) y futura (TO BE) del proceso de Gestión de Proyectos. El objetivo general es elaborar una propuesta de arquitectura empresarial enfocada en la mejora y optimización del proceso de Gestión de Proyectos de la empresa para un mejor desempeño de la organización y asegurar su crecimiento en el mercado tecnológico.

En el primer capítulo, se presenta a la empresa que es el objeto de estudio, los objetivos y beneficios del proyecto.

En el segundo capítulo, se explica sobre la fundamentación del marco teórico en donde se detallan los conceptos principales que ayudan a comprender las diferentes herramientas a utilizar.

En el tercer capítulo, se desarrolla la Arquitectura Empresarial bajo el marco de referencia TOGAF (The Open Group Architecture Framework), se definen las limitaciones, problemas y principios de arquitectura, se desarrolla una arquitectura de línea base (AS IS) y se propone una arquitectura destino (TO BE), que como resultado se obtiene el análisis de brechas; y finalmente se desarrolla un cuadro resumen del plan de migración. Además aborda las metodologías ágiles de desarrollo, en el cual identifican los grupos de trabajo, dinámicas propuestas y definición de herramienta a utilizar.

El cuarto capítulo define la propuesta del proyecto, la cual integre los capítulos mencionados anteriormente.

En último lugar, para finalizar el documento, se detalla la presentación de las conclusiones, recomendaciones, glosario de términos, siglario, bibliografía y anexos.

Palabras clave: Arquitectura Empresarial, marco de referencia TOGAF, marco teórico, metodologías ágiles de desarrollo, proceso de Gestión de Proyectos 


\section{(1)}

\section{SUMMARY}

This professional project develops the proposal for the implementation of an Enterprise Architecture for a software consulting firm, for which the analysis of the current (AS IS) and future (TO BE) situation of the Project Management process has been carried out. The overall objective is to develop an enterprise architecture proposal focused on the enhancement and optimization of the Project Management process of the company in order to get a better performance of the organization and ensure its growth in the technology market.

The first chapter presents the company, which is the object of study, as well as the objectives and benefits of the project.

The second chapter explains the grounds for the theoretical framework, detailing the main concepts that help understand the different tools to be used.

The third chapter develops the Business Architecture under the TOGAF(The Open Group Architecture Framework) reference framework; defines the architecture limitations, issues and principles; develops the baseline architecture(AS IS) and proposes a target architecture (TO BE), resulting in a gap analysis; and finally develops a summary table of the migration plan. In addition, it addresses agile development methodologies, in which workgroups, proposed dynamics and tool definition to be used are identified.

The fourth chapter defines the project proposal, which integrates the chapters mentioned above.

Finally, the presentation of conclusions, recommendations, glossary of terms, glossary of acronyms, bibliography and annexes is detailed.

Keywords: Enterprise Architecture, reference framework TOGAF, theoretical framework, agile development methodologies, Project Management process. 


\section{() \\ ÍNDICE}

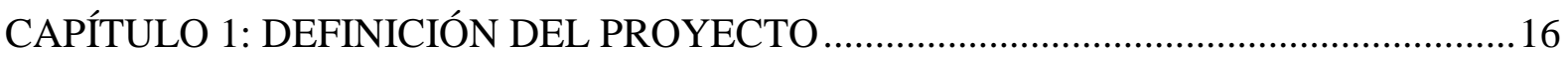

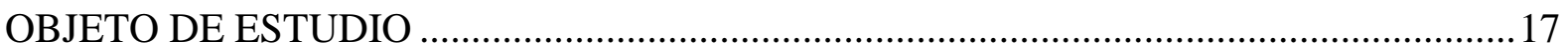

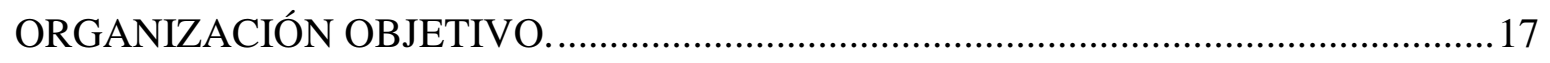

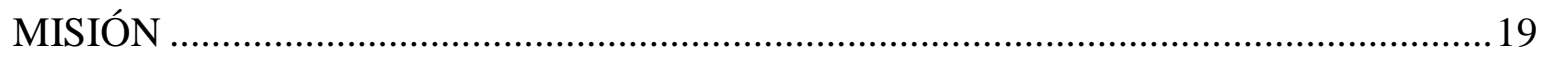

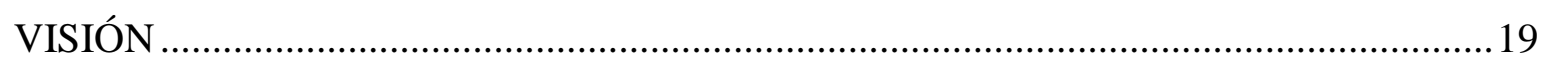

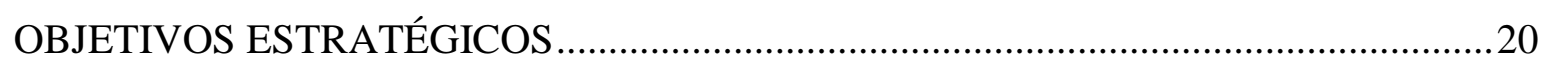

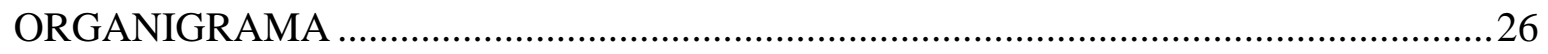

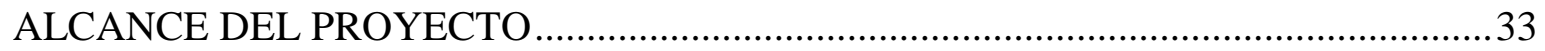

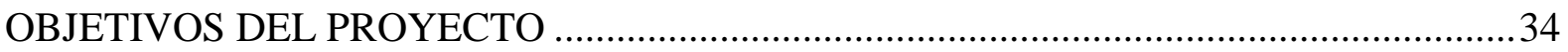

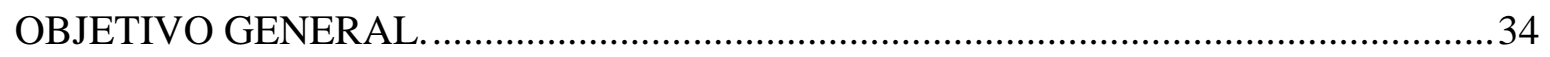

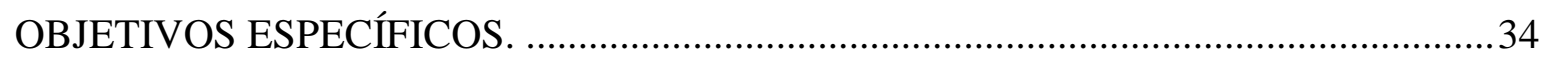

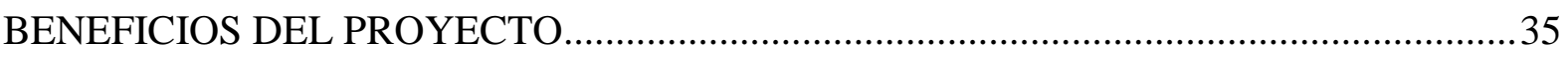

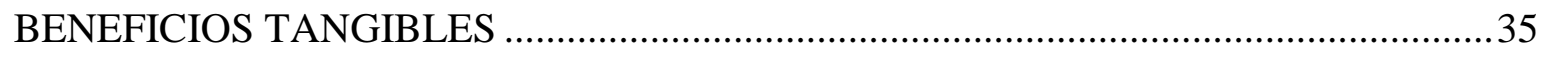

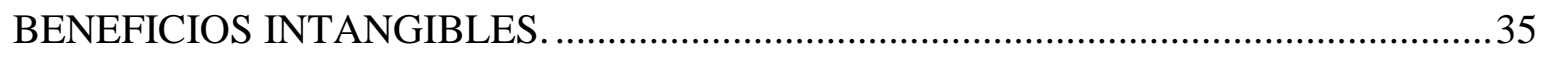

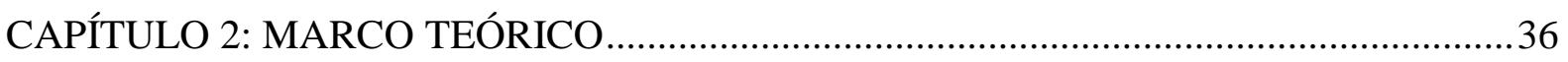

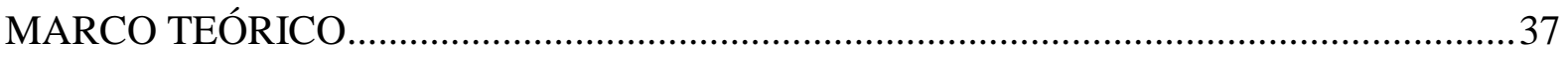

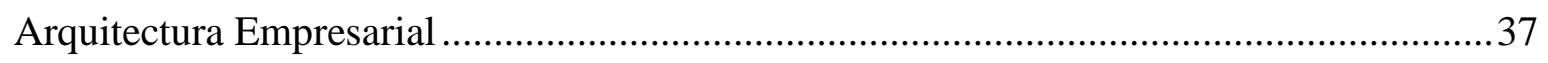

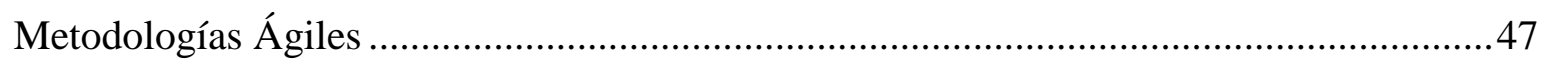

JIRA Software

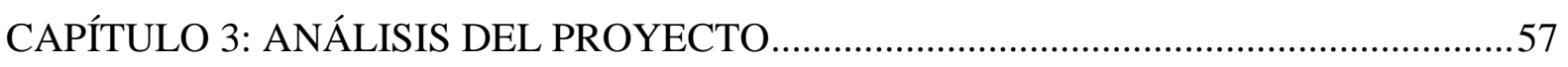

ARQUITECTURA EMPRESARIAL ..............................................................................5

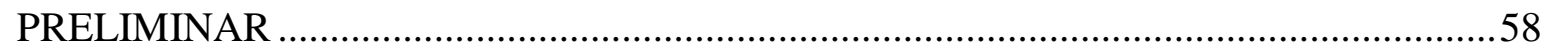

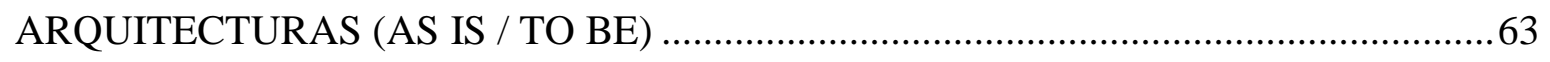

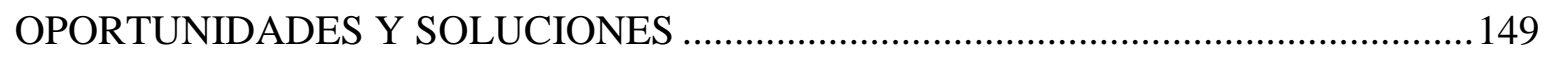

MÉTODOS ÁGILES PARA EL DESARROLLO DE SOFTWARE .....................................154

IDENTIFICACIÓN DE FORTALEZAS Y DEBILIDADES ………………………….....154

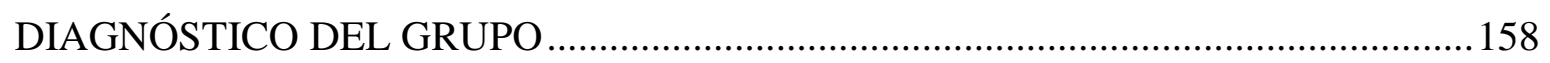

IDENTIFICACIÓN DE LAS DINÁMICAS PROPUESTAS............................................. 160

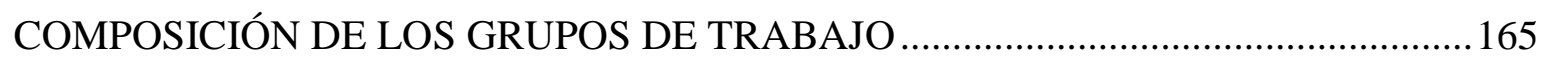




\section{(9)}

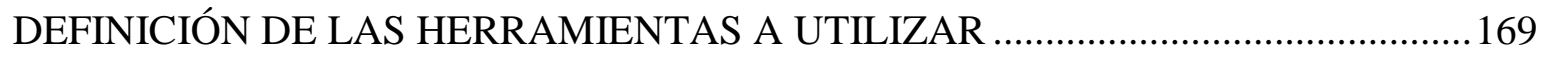

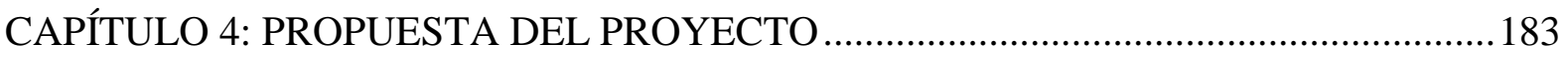

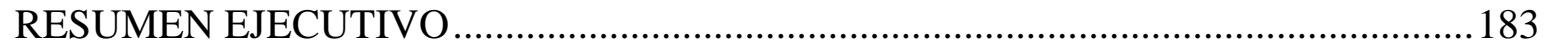

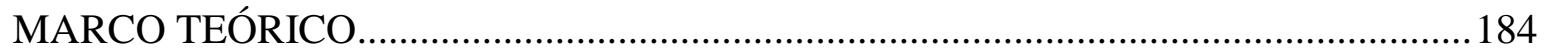

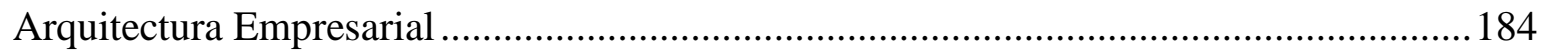

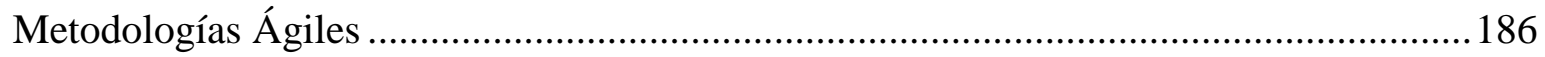

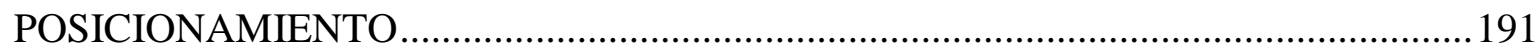

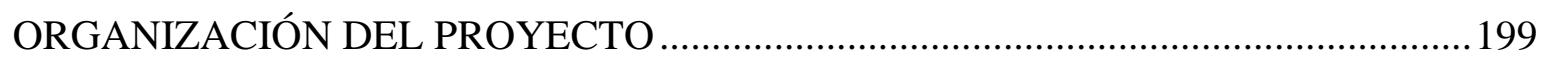

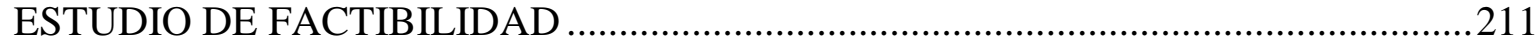

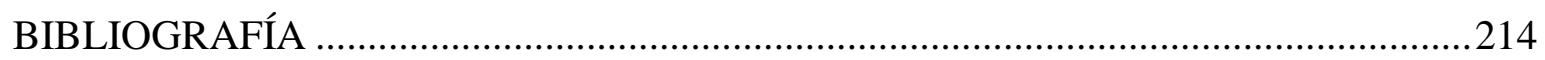

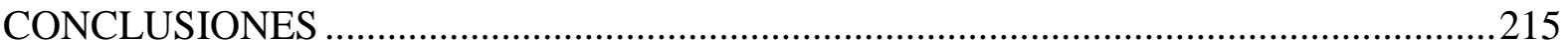

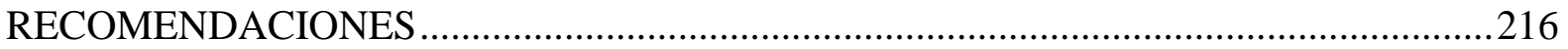

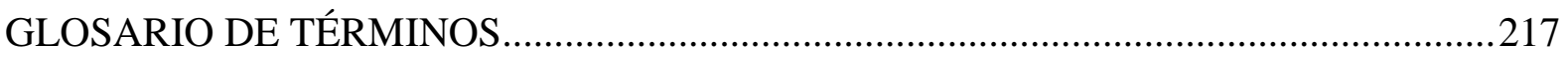

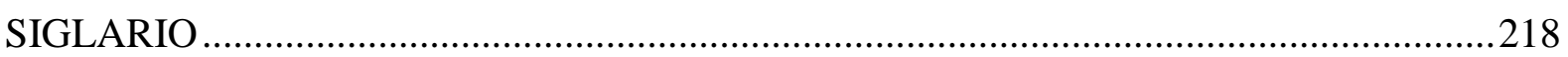

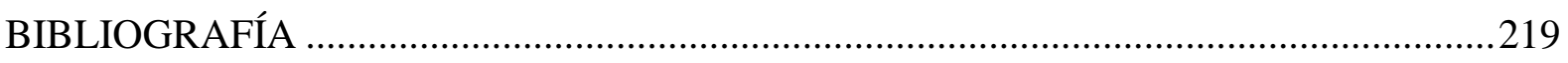

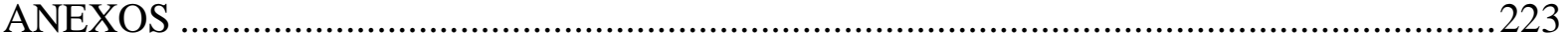




\section{(1)}

\section{ÍNDICE DE FIGURAS}

Figura 1. Mapa de procesos de la empresa (ASIS). Adaptado de "Manual de calidad", por El Objeto de Estudio, 2017.

Figura 2. Organigrama de la empresa. Adaptado de "Manual de funciones y perfiles de puestos", por El Objeto de Estudio, 2017.

Figura 3. Entorno y enfoques de Arquitectura Empresarial y de TI. Adaptado de "Enfoque de arquitectura de solución, mecanismo para reducir la brecha entre la arquitectura empresarial y la implementación de soluciones tecnológicas”, por Revista de Ingenería Dyna, 2015. 38

Figura 4. Metodologías y frameworks de arquitectura empresarial. Adaptado de "¿Qué es Arquitectura Empresarial?”, por Adriana Molano, 2015

Figura 5. Ciclo de desarrollo de arquitectura. Adaptado de "Part II - Architecture

Development Method", por The Open Group, 1999 - 2018. .45

Figura 6. Metodologías tradicionales vs metodologías ágiles. Adaptado de "Revisión de metodologías ágiles para el desarrollo de software”, por Prospectiva, 2013. .48

Figura 7. Planificación ágil vs tradicional. Adaptado de "Planificación ágil vs planificación tradicional", por Proyectosagiles.org, 2010.

Figura 8. Equipo Scrum. Adaptado de "Scrum roles y responsabilidades", por Kenneth Rubin, 2018. .51

Figura 9. Fases de un Sprint. Adaptado de "Metodología Scrum: Fases de un Sprint", por Andrés Navarro, Juan Fernández \& Juan Morales, 2013. .52

Figura 10. Las reglas del SCRUM. Adaptado de "Scrum Manager I: Las reglas de scrum", por Juan Palacio, 2015. .54

Figura 11. Organigrama de la empresa - Proceso Seleccionado (ASIS). Adaptado de "Manual de funciones y perfiles de puestos", por El Objeto de Estudio, 2017. .68

Figura 12. Mapa de procesos de la empresa (ASIS). Adaptado de "Manual de calidad", por El Objeto de Estudio, 2017. .71 


\section{$(1)$}

Figura 13. Diagrama de Actividades del Subproceso de Control y Seguimiento de Proyectos (ASIS). Adaptado de "Procedimiento de Gestión de Proyecto", por El Objeto de Estudio, 2017 .77

Figura 14. Diagrama de Actividades del Subproceso de Gestión de Proyectos (ASIS). Adaptado de "Procedimiento de Gestión de Proyecto", por El Objeto de Estudio, 2017.

Figura 15. Modelo de Datos (ASIS). Adaptado de "Manual de Configuración", por El Objeto de Estudio, 2017.

Figura 16. Diagrama de Aplicaciones del Proceso de Gestión de Proyectos (ASIS). Adaptado de "Manual de Configuración", por El Objeto de Estudio, 2017. 100

Figura 17. Diagrama de Componentes del Proceso de Gestión de Proyectos (ASIS). Adaptado de "Manual de Configuración”, por El Objeto de Estudio, 2017. 101

Figura 18. Diagrama de Distribución Física de Equipos de Red (ASIS). Adaptado de "Manual de Configuración”, por El Objeto de Estudio, 2017. 106

Figura 19. Organigrama de la empresa - Proceso Seleccionado (TOBE). Adaptado de "Manual de funciones y perfiles de puestos", por El Objeto de Estudio, 2017. 109

Figura 20. Mapa de procesos de la empresa (TOBE). Adaptado de "Manual de calidad", por

El Objeto de Estudio, 2017.

Figura 21. Diagrama de Actividades del Subproceso de Control y Seguimiento de Proyectos (TOBE). Adaptado de "Procedimiento de Gestión de Proyecto", por El Objeto de Estudio, 2017. 118

Figura 22. Diagrama de Actividades del Subproceso de Gestión de Proyectos (TOBE). Adaptado de "Procedimiento de Gestión de Proyecto", por El Objeto de Estudio, 2017.

Figura 23. Modelo de Datos (TOBE). Elaboración propia.

Figura 24. Diagrama de Aplicaciones del Proceso de Gestión de Proyectos (TOBE). Elaboración propia.

Figura 25. Diagrama de Componentes del Proceso de Gestión de Proyectos (TOBE). Elaboración propia.

Figura 26. Diagrama de Distribución Física de Equipos de Red (TOBE). Adaptado de “Manual de Operación”, por El Objeto de Estudio, 2017. 138 


\section{(1)}

Figura 27. Estructura de desglose de trabajo. Adaptado de "Welcome to the TOGAF® Standard, Version 9.2, a standard of The Open Group”, por The Open Group, 1999 2018

Figura 28. Prototipo del Tablero Scrum para el equipo de desarrollo. Elaboración Propia. 180

Figura 29. Prototipo del Tablero Scrum para el equipo de calidad. Elaboración Propia ...... 180

Figura 30. Lista de Personalización Proyecto Base. Adaptado de "Procedimiento de

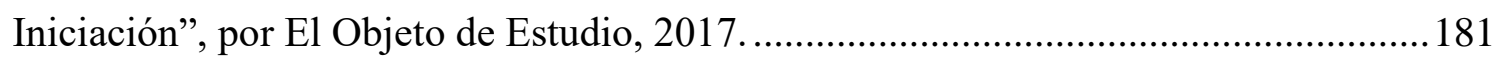

Figura 31. Lista de Personalización Proyecto Scrum. Elaboración propia........................... 182

Figura 32. Formula del VAN. Adaptado de "Las Decisiones de Presupuesto de Capital:

Criterios Financieros Fundamentales”, por Alarcòn, Ullua y Carretero, 2014...... 189

Figura 33. Variables del VAN. Adaptado de "Las Decisiones de Presupuesto de Capital: Criterios Financieros Fundamentales”, por Alarcòn, Ullua y Carretero, 2014. 189

Figura 34. Formula del TIR. Adaptado de "Las Decisiones de Presupuesto de Capital: Criterios Financieros Fundamentales”, por Alarcòn, Ullua \& Carretero, 2014. 190

Figura 35. Variables del TIR. Adaptado de "Las Decisiones de Presupuesto de Capital: Criterios Financieros Fundamentales”, por Alarcòn, Ullua \& Carretero, 2014. 190

Figura 36. Mapa de procesos de la empresa. Adaptado de "Manual de calidad", por El Objeto de Estudio, 2017. 191

Figura 37. Costos del proyecto. Elaboración Propia ............................................................ 198

Figura 38. Cuadro del total ahorrado por recurso. Elaboración Propia................................211

Figura 39. Cuadro de Flujo de Inversión. Elaboración Propia.....

Figura 40. Carta de Aprobación Objeto de Estudio. Adaptado de "Carta de aprobación de tema de tesis", por UPC, 2018.

Figura 41. Reducción de la actualización y procesamiento de cronogramas. Elaboración propia. .224

Figura 42. Reducción de no conformidades. Elaboración propia. .224 


\section{(1)}

\section{ÍNDICE DE TABLAS}

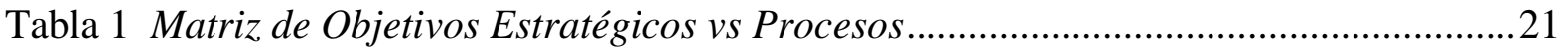

Tabla 2 Objetivos de los Procesos de Negocios ......................................................................23

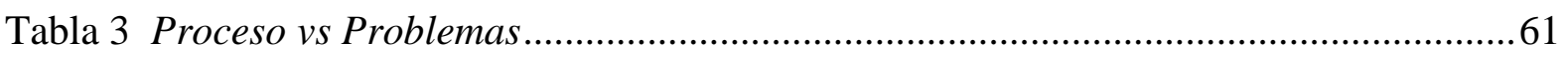

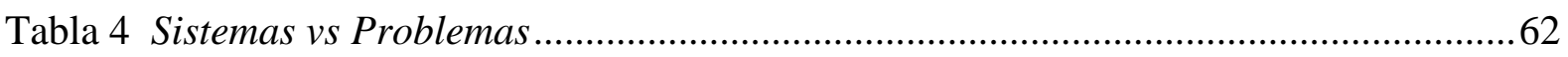

Tabla 5 Principio de Negocio - Satisfacción del cliente ....................................................... 64

Tabla 6 Principio de Negocio - Creación y actualización de productos ................................. 64

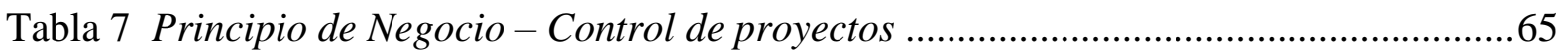

Tabla 8 Principio de Datos - Información de procesos compartido ........................................65

Tabla 9 Principio de Datos - Bitácora de seguimiento de proyectos.......................................66

Tabla 10 Principio de Datos - Copia de seguridad de información .......................................66

Tabla 11 Principio de Aplicaciones - Facilidad de uso .........................................................66

Tabla 12 Principio de Tecnología - Estándares y mejores prácticas ....................................67

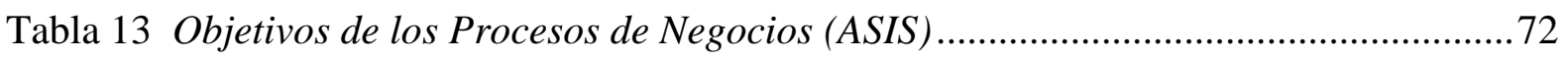

Tabla 14 Matriz de Objetivos Estratégicos vs Procesos (ASIS) .............................................76

Tabla 15 Actividades del Subprocesos de Control y Seguimiento de Proyectos (ASIS)......... 79

Tabla 16 Actividades del Subprocesos de Gestión de Proyectos (ASIS) ..................................8 80

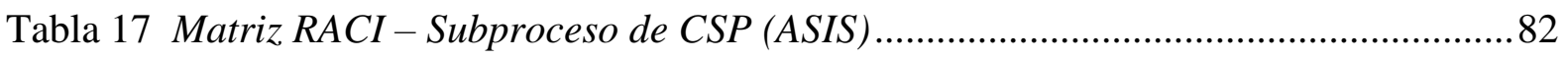

Tabla 18 Matriz RACI - Subproceso de Gestión de Proyectos (ASIS) .....................................82

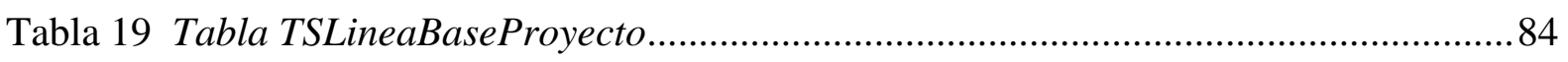

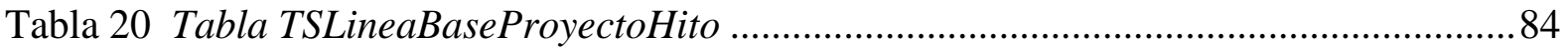

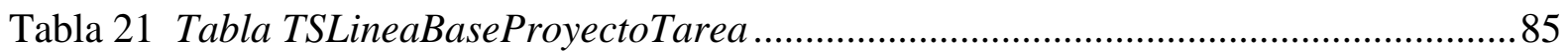

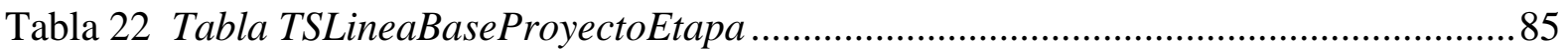

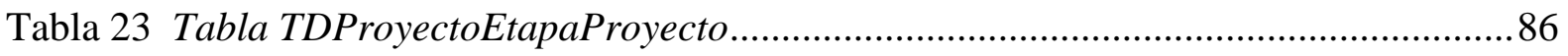

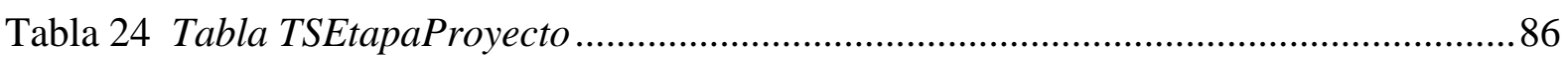

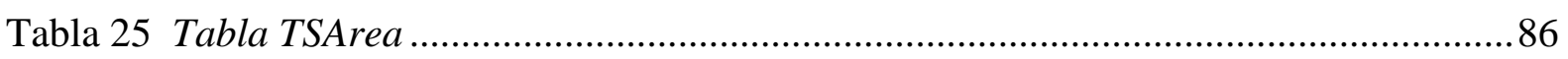

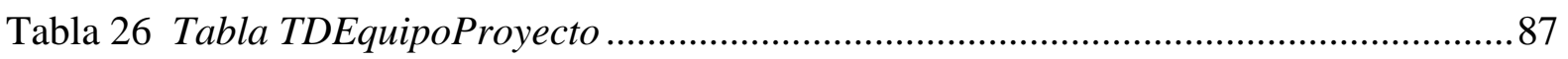

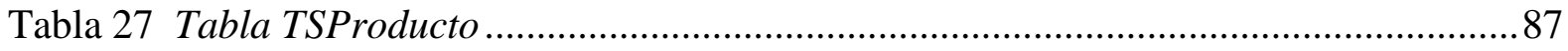




\section{(1)}

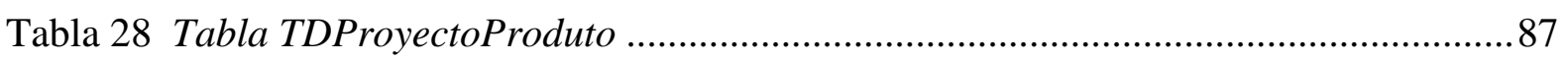

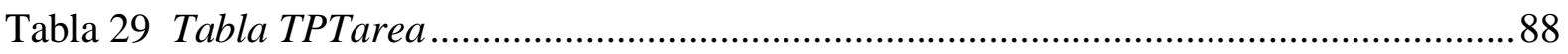

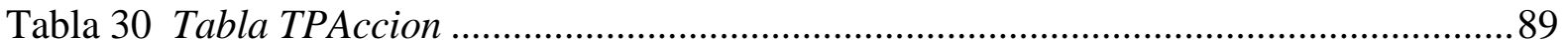

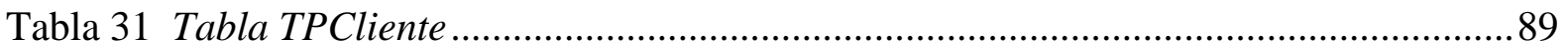

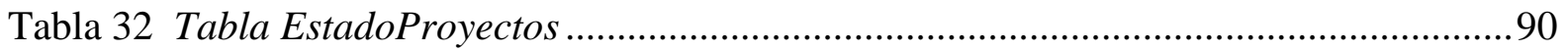

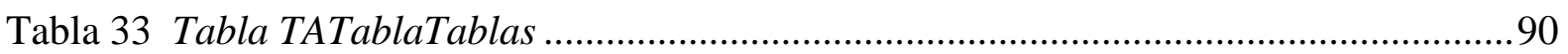

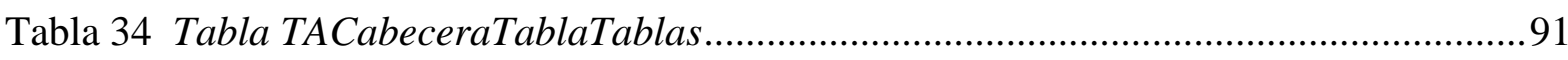

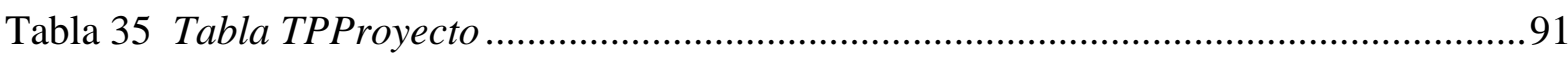

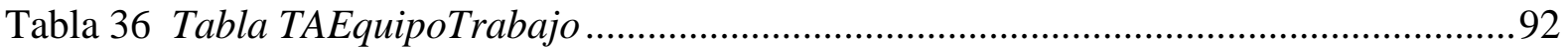

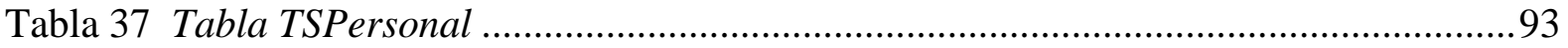

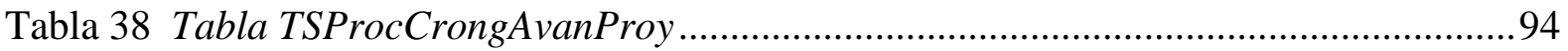

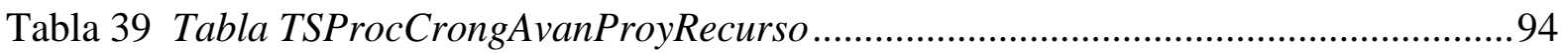

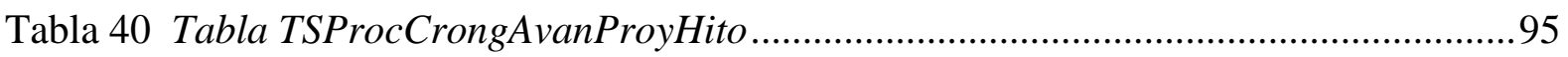

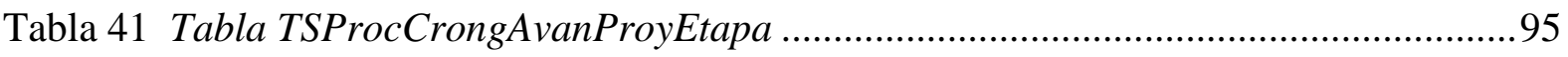

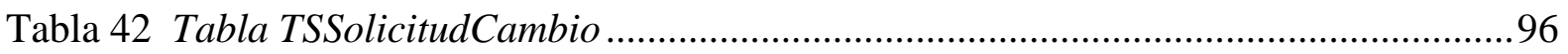

Tabla 43 Matriz de Entidades de Datos vs Procesos del Negocio (ASIS) .............................. 98

Tabla 44 Detalle de Aplicaciones del Proceso Gestión de Proyectos (ASIS)........................ 100

Tabla 45 Detalle de Componentes del Proceso Gestión de Proyectos (ASIS) ..................... 101

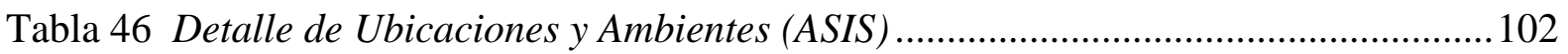

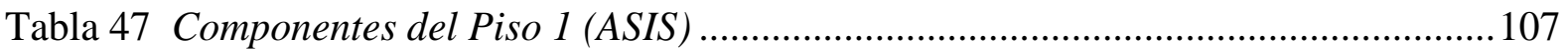

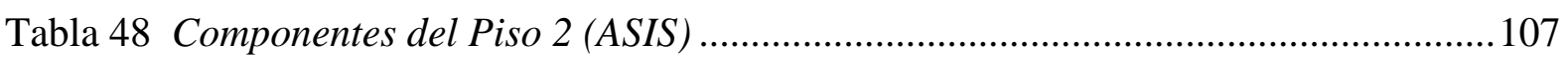

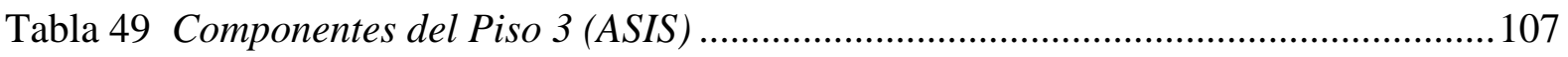

Tabla 50 Objetivos de los Procesos de Negocios (TOBE) ................................................ 113

Tabla 51 Matriz de Objetivos Estratégicos vs Procesos (TOBE) …................................... 117

Tabla 52 Actividades del Subprocesos de Control y Seguimiento de Proyectos (TOBE) ....120

Tabla 53 Actividades del Subprocesos de Gestión de Proyectos (TOBE) .............................121

Tabla 54 Matriz RACI - Subproceso de CSP (TOBE) .......................................................... 123

Tabla 55 Matriz RACI - Subproceso de Gestión de Proyectos (TOBE)................................ 123

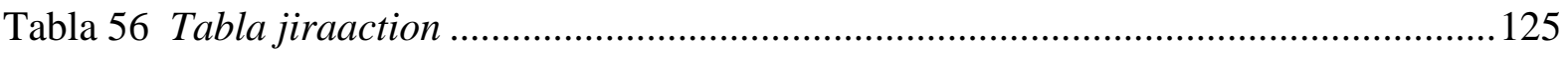

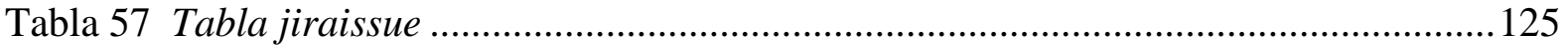

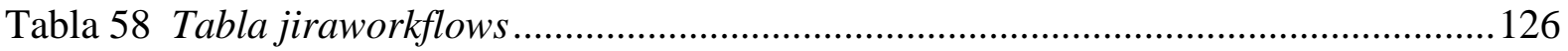

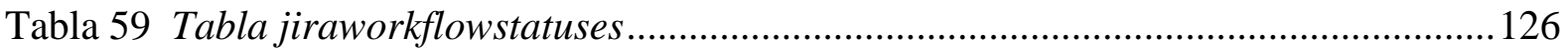




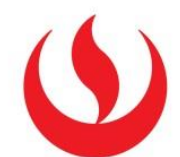

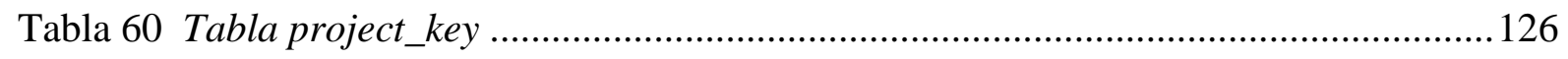

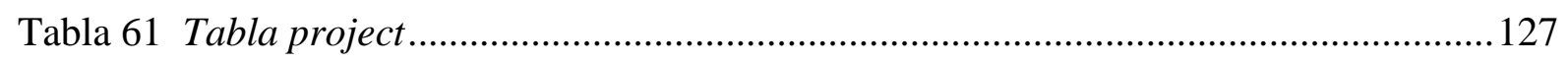

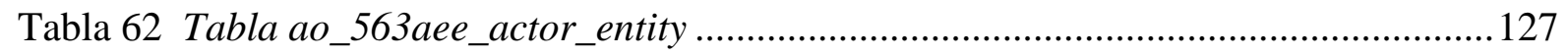

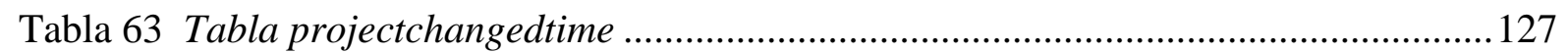

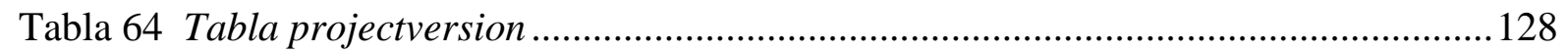

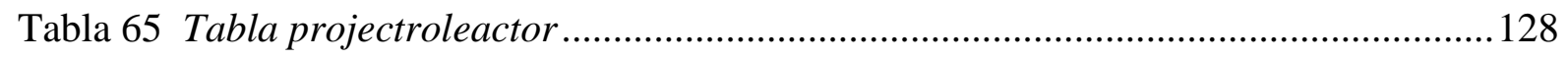

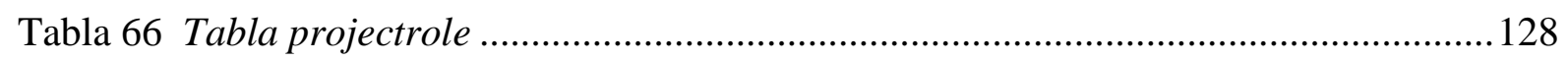

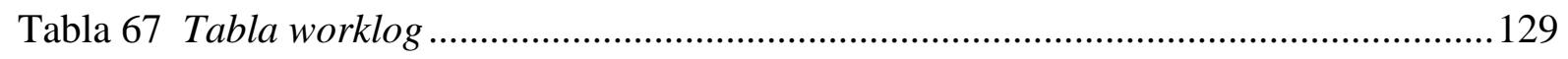

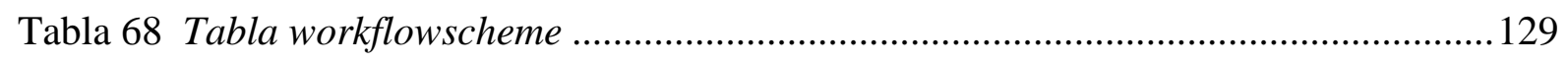

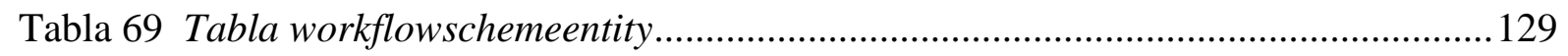

Tabla 70 Matriz de Entidades de Datos vs Procesos del Negocio (TOBE)...........................130

Tabla 71 Detalle de Aplicaciones del Proceso Gestión de Proyectos (TOBE) ...................... 132

Tabla 72 Detalle de Componentes del Proceso Gestión de Proyectos (TOBE) .....................133

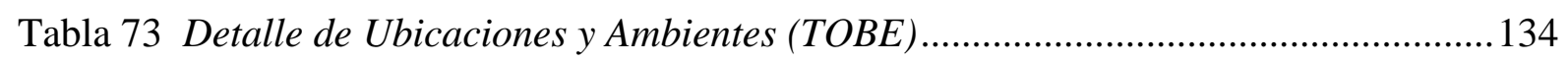

Tabla 74 Análisis de brechas - Arquitectura de Negocio del Primer Subproceso ................ 139

Tabla 75 Análisis de brechas - Arquitectura de Negocio del Segundo Subproceso .............. 140

Tabla 76 Análisis de brechas de la Arquitectura de Aplicación ............................................ 142

Tabla 77 Análisis de brechas de la Arquitectura Tecnológica .............................................. 143

Tabla 78 Análisis de brechas de la Arquitectura de Datos .................................................. 144

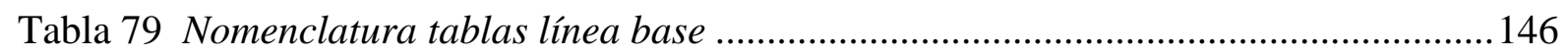

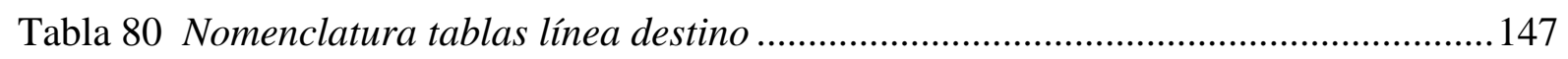

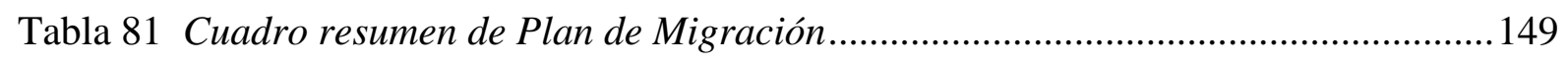

Tabla 82 Cuadro del Enfoque de secuencia de Implementación .........................................152

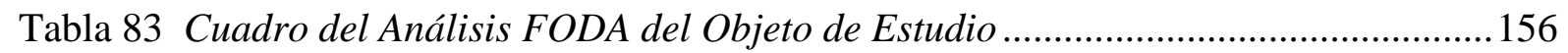

Tabla 84 Cuadro del Análisis de Fortalezas y Debilidades del grupo de trabajo ................ 157

Tabla 85 Cuadro del Análisis de Debilidades y Posibles Problemas..................................... 159

Tabla 86 Cuadro de Ejecución y beneficios del Sprint Planning ......................................... 160

Tabla 87 Cuadro de Ejecución y beneficios del Daily Scrum .............................................. 161

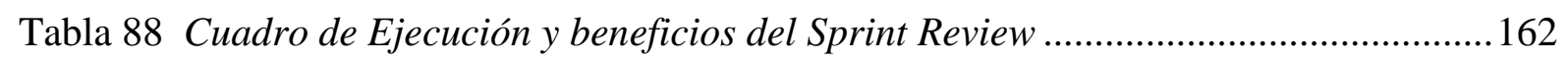

Tabla 89 Cuadro de Ejecución y beneficios del Sprint Retrospective ................................. 162

Tabla 90 Cuadro de Ejecución y beneficios del Planning Poker .......................................... 163

Tabla 91 Cuadro de Ejecución y beneficios de las Capacitaciones funcionales internas.... 164 
Tabla 92 Cuadro de habilidades del Product Owner (Objeto de Estudio). 165

Tabla 93 Cuadro de habilidades del Scrum Master (Proveedor) ........................................... 166

Tabla 94 Cuadro de habilidades del Scrum Master (Objeto de Estudio)............................. 167

Tabla 95 Cuadro de habilidades del Development Team (Proveedor) ...............................167

Tabla 96 Cuadro de habilidades del Quality Team (Objeto de Estudio) ..............................168

Tabla 97 Cuadro de Costo Total del Equipo ......................................................................... 169

Tabla 98 Cuadro de Criterios para el Equipo de Desarrollo.............................................. 170

Tabla 99 Cuadro de Criterios para el Equipo de Calidad .................................................. 170

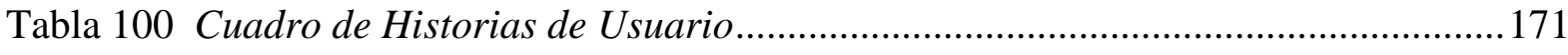

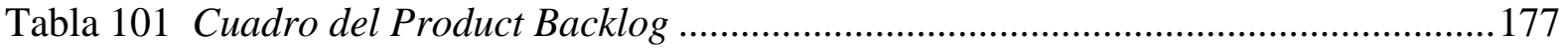

Tabla 102 Cuadro del Sprint Backlog del Sprint 1 ............................................................. 179

Tabla 103 Cuadro de Beneficios del Marco de Trabajo SCRUM .........................................188

Tabla 104 Objetivo del Proceso de Gestión de Proyectos...................................................... 192

Tabla 105 Cuadro de problemas del proceso de Gestión de Proyectos .................................192

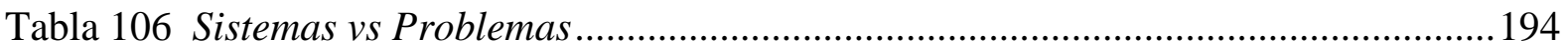

Tabla 107 Cuadro de Indicadores de éxito del proyecto .................................................... 195

Tabla 108 Cuadro del Equipo del Proyecto ....................................................................... 199

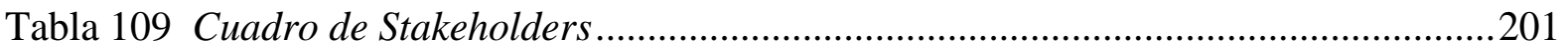

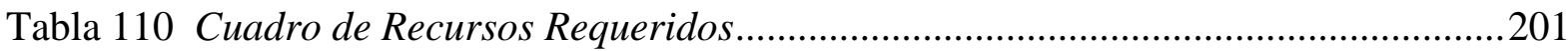

Tabla 111 Cuadro de Fases e hitos del proyecto de Mejora del procedimiento de Gestión de

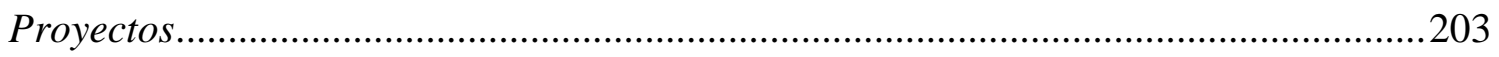

Tabla 112 Cuadro de Fases e hitos del proyecto de Implementación Software JIRA ..........206

Tabla 113 Cuadro de Riesgos y Mitigación ............................................................................210 


\section{(1)}

\section{INTRODUCCIÓN}

Debido a la evolución constante de tecnologías, cambios normativos, certificaciones, servicios, e infraestructura debemos estar preparados a los cambios e ir a la vanguardia con las últimas novedades con las que este rubro se enfrenta a nivel nacional como mundial. Por ello, las organizaciones deben otorgar mayor esfuerzo hacia la automatización y mejoras de sus procesos mediante el uso de estándares, y mejores prácticas con el fin de cumplir con los objetivos estratégicos.

El presente proyecto profesional tiene como objetivo presentar la propuesta de arquitectura empresarial para una consultora de software enfocada en la mejora y optimización del proceso de Gestión de Proyectos. Los problemas han sido identificados y se plantea una solución integral que va de la mano con actualización del procedimiento y renovación tecnológica de los aplicativos. Para dar soporte a esta problemática se decidió utilizar el marco de trabajo TOGAF $^{1}$ como framework de Arquitectura Empresarial.

Después de haber aplicado TOGAF y de haber identificado las brechas y sus posibles soluciones, se optó por aplicar el framework de Scrum para el proyecto de desarrollo de software. En esta sección se contemplará los roles, eventos, artefactos propios de éste, su modo del cómo se va aplicar y los beneficios que brindará al proyecto.

Por último, el desarrollo de esta propuesta nos ha permitido ampliar nuestros conocimientos sobre frameworks, aplicaciones, metodologías y sobre cómo desarrollar una solución de arquitectura empresarial.

\footnotetext{
${ }^{1}$ Para ver el significado de "TOGAF" ir a la sección SIGLARIO del documento.
} 
CAPÍTULO 1: DEFINICIÓN DEL PROYECTO 


\section{(1)}

\section{OBJETO DE ESTUDIO}

\section{ORGANIZACIÓN OBJETIVO.}

La consultora de software está dedicada a proveer servicios de desarrollo de aplicaciones, de integración de sistemas, de gerencia de proyectos y de consultoría.

Las soluciones que provee generalmente se enmarcan en el desarrollo de aplicaciones bajo arquitectura Cliente/Servidor, de Sistemas Distribuidos y de Internet (Web), utilizando tecnología de bases de datos, de seguridad, de comunicaciones y de ambiente visual. El conocimiento y experiencia de su personal así como la permanente labor de investigación y desarrollo que realiza, le ha permitido brindar soluciones de teleacceso ${ }^{2}$ de clientes, de integración de ambientes multiplataforma así como la implementación de sistemas institucionales, en los sectores de telecomunicaciones, comercial, financiero y gubernamental. A través de los productos que ha desarrollado, se ha posicionado como el líder en el mercado de integración de sistemas y transferencia electrónica de fondos. Al respecto, el Objeto de Estudio (2017) señala que entre los principales productos se encuentran:

- Teleacceso de Clientes (SIX/TCL)

El SIX/TCL ${ }^{3}$ tiene la funcionalidad de operar en un ambiente cliente/servidor y administra una serie de funciones que permiten que el programa aplicativo cliente pueda solicitar una determinada serie de servicios al SIX/TCL server. Los servicios a ser brindados en el sistema son atendidos por programas servidores que pueden convivir con el SIX/TCL o residir en el host de la institución o en otros computadores, actuando el SIX/TCL como un gateway ${ }^{4}$ La arquitectura

\footnotetext{
${ }^{2}$ Para ver la definición de "teleacceso" ir a la sección GLOSARIO del documento.

${ }^{3}$ Para ver el significado de "SIX/TCL" ir a la sección SIGLARIO del documento.

${ }^{4}$ Para ver la definición de "gateway" ir a la sección GLOSARIO del documento.
} 


\section{(1)}

del SIX/TCL admite realizar intercambio de archivos, envío de jobs ${ }^{5}$ remotos, transacciones en línea e incluso la operación del sistema en modo off-host ${ }^{6}$. El SIX/TCL cubre una gran variedad de aplicaciones en el ámbito empresarial, entre las cuales podemos destacar:

$\checkmark \quad$ Servicios de acceso remoto a su información.

$\checkmark$ Integración de operaciones entre clientes y proveedores en un comercio electrónico interempresarial.

$\checkmark \quad$ Establecer en la forma de mensajes, datos o imágenes el intercambio de información a través de redes privadas.

\section{- Transferencia Electrónica de Fondos Switch (SIX/EFT Switch)}

Es una plataforma de servicios transaccionales financieros basada en el estándar ISO ${ }^{7}$ 8583, denominada SIX/EFT Switch ${ }^{8}$. El cual provee una infraestructura que permite desplegar en forma modular sus aplicaciones de misión crítica. SIX/EFT Switch establece un grupo de funciones que son requeridas para brindar los servicios de Cajeros Automáticos, Internet Banking, otros y asimismo se pueden integran otras aplicaciones propietarias. Aparte de ello, soporta una gran variedad de aplicaciones de servicios de pago y banca electrónica, así como de intercambio. Entre sus múltiples usos podemos destacar los siguientes:

$\checkmark$ Empresas que quieran servicios de banca electrónica, tales como terminales de consulta y de ventanilla, cajeros automáticos, puntos de venta, entre otros.

Empresas que quieran implementar una red de intercambio de transacciones financieras, procedente de múltiples dispositivos electrónicos.

\footnotetext{
${ }^{5}$ Para ver la definición de "job” ir a la sección GLOSARIO del documento.

${ }^{6}$ Para ver la definición de "off-host" ir a la sección GLOSARIO del documento.

${ }^{7}$ Para ver el significado de "ISO" ir a la sección SIGLARIO del documento.

${ }^{8}$ Para ver el significado de "SIX/EFT Switch" ir a la sección SIGLARIO del documento.
} 


\section{(1)}

$\checkmark$ Empresas que quieran conectarse en forma rápida y segura a una red de intercambio de transacciones.

$\checkmark$ Empresas que quieran ampliar su alcance de servicio y volumen de transacciones implementado una red de intercambio de transacciones.

Los principales beneficios que nos brinda el SIX/EFT Switch son los siguientes:

$\checkmark$ Contar con soporte local

$\checkmark$ Costos mínimos de operación y autonomía en la implantación de sus servicios.

$\checkmark$ Las redes de intercambio a nivel nacional e internacional se integran rápidamente.

$\checkmark$ Confiabilidad e Integridad de las operaciones

$\checkmark \quad$ Establece una arquitectura integral para brindar sus servicios de Pagos y de Banca Electrónica

$\checkmark$ Definición de transacciones en forma paramétrica (pp. 4-6).

\section{MISIÓN}

"Brindar soluciones y servicios transaccionales innovadores que transformen el mundo en que vivimos" (Objeto de Estudio, 2017, p.7).

\section{VISIÓN}

"Ser la empresa de TI especializada en soluciones y servicios transaccionales, líder en Latinoamérica.

Rentable y Eficiente

Profesionales especializados y de experiencia

Estándares y mejores prácticas de la industria

Promotor del desarrollo tecnológico del país” (Objeto de Estudio, 2017, p.8). 


\section{(}

\section{OBJETIVOS ESTRATÉGICOS}

Los objetivos estratégicos son los objetivos planteados por una organización para así lograr determinadas metas. Al respecto, el Objeto de Estudio (2017) señala que:

1. Objetivo Estratégico 1: OE1: Contar con un índice de Satisfacción Global por Cliente en un 82\%. (Servicio integral: Soporte, Actividades, Proyectos).

2. Objetivo Estratégico 2: OE2: Lograr que el 90\% de personal del área de Operaciones tenga como mínimo 25 horas de capacitación orientadas al logro del incremento de la productividad.

3. Objetivo Estratégico 3: OE3: Crear por lo menos 2 nuevos productos competitivos durante el año, y actualizar 2 de los actuales.

4. Objetivo Estratégico 4: OE4: Invertir el 8\% de las ventas en la investigación y desarrollo tecnológico orientado a la productividad y competitividad.

5. Objetivo Estratégico 5: OE5: Incrementar en un 10\% la productividad mediante el mejoramiento de nuestros procesos (menos errores y velocidad de ejecución). (p. 16). 


\section{(}

Tabla 1

Matriz de Objetivos Estratégicos vs Procesos

\begin{tabular}{|c|c|c|c|c|c|c|c|c|c|c|c|c|c|c|c|c|c|c|c|c|c|c|c|c|c|c|c|}
\hline \multirow{3}{*}{$\begin{array}{l}\text { Objetivos } \\
\text { Estratégicos }\end{array}$} & \multicolumn{27}{|c|}{ PROCESOS } \\
\hline & \multicolumn{3}{|c|}{ Estratégicos } & \multicolumn{13}{|c|}{ Aроуо } & \multicolumn{11}{|c|}{ Claves } \\
\hline & 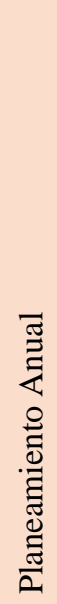 & 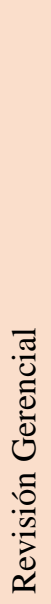 & 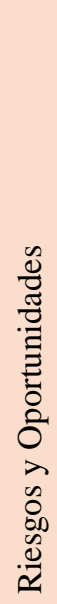 & 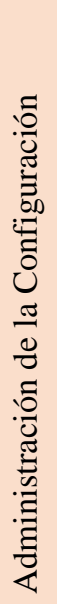 & 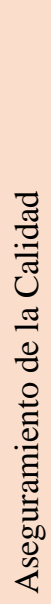 & 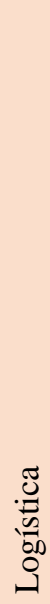 & 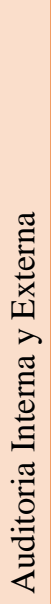 & 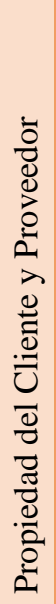 & 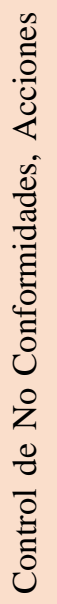 & 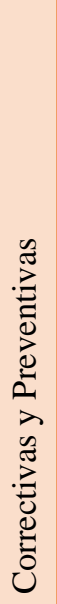 & 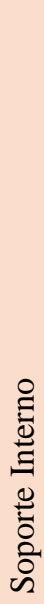 & $\begin{array}{l}0 \\
0 \\
0 \\
0 \\
0 \\
0 \\
2 \\
0 \\
0 \\
0 \\
0 \\
0 \\
0 \\
0 \\
0 \\
0 \\
0 \\
0 \\
0 \\
0\end{array}$ & 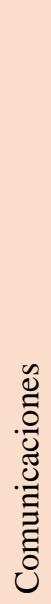 & 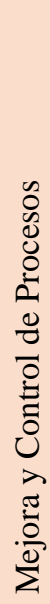 & 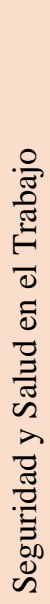 & 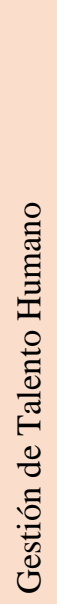 & 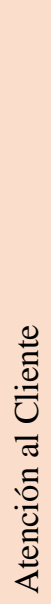 & 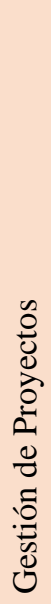 & $\begin{array}{l}\frac{0}{0} \\
\frac{\pi}{0} \\
\frac{\pi}{\Xi} \\
\end{array}$ & 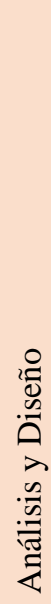 & 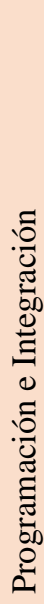 & 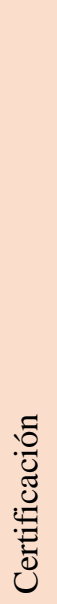 & 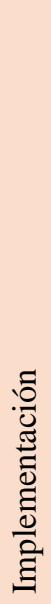 & 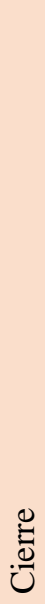 & 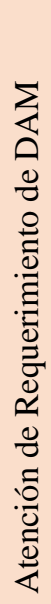 & 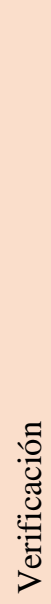 & $\begin{array}{l}\frac{\pi}{0} \\
\sum_{0}^{0} \\
0 \\
0\end{array}$ \\
\hline OE 1 & & $X$ & & & & & & & & & & & & & & & $\mathrm{X}$ & $X$ & & & & & & & & & \\
\hline OE 2 & $\mathrm{X}$ & & & & & & & & & & & & & & & $\mathrm{X}$ & & & & & & & & & & & \\
\hline OE 3 & & & & & & & & & & & & & & & & & & $X$ & & & & & & & $X$ & & \\
\hline OE 4 & $\mathrm{X}$ & $X$ & & & & $X$ & & & & & & & & & & & & & & & & & & & & & \\
\hline OE 5 & $\mathrm{X}$ & & & & & & & & & & & & & & & & & $X$ & $\mathrm{X}$ & $\mathrm{X}$ & $\mathrm{X}$ & $\mathrm{X}$ & $X$ & $X$ & $X$ & $X$ & $X$ \\
\hline
\end{tabular}

Nota: Se ha realizado el cruce entre los objetivos estratégicos de la empresa y sus procesos para poder identificar cuales están relacionados. Adaptado de "Manual de Calidad 2017”, por El Objeto de Estudio, 2017. 


\section{(1)}

○ MAPA DE PROCESOS Y FUNCIONES DE NEGOCIO

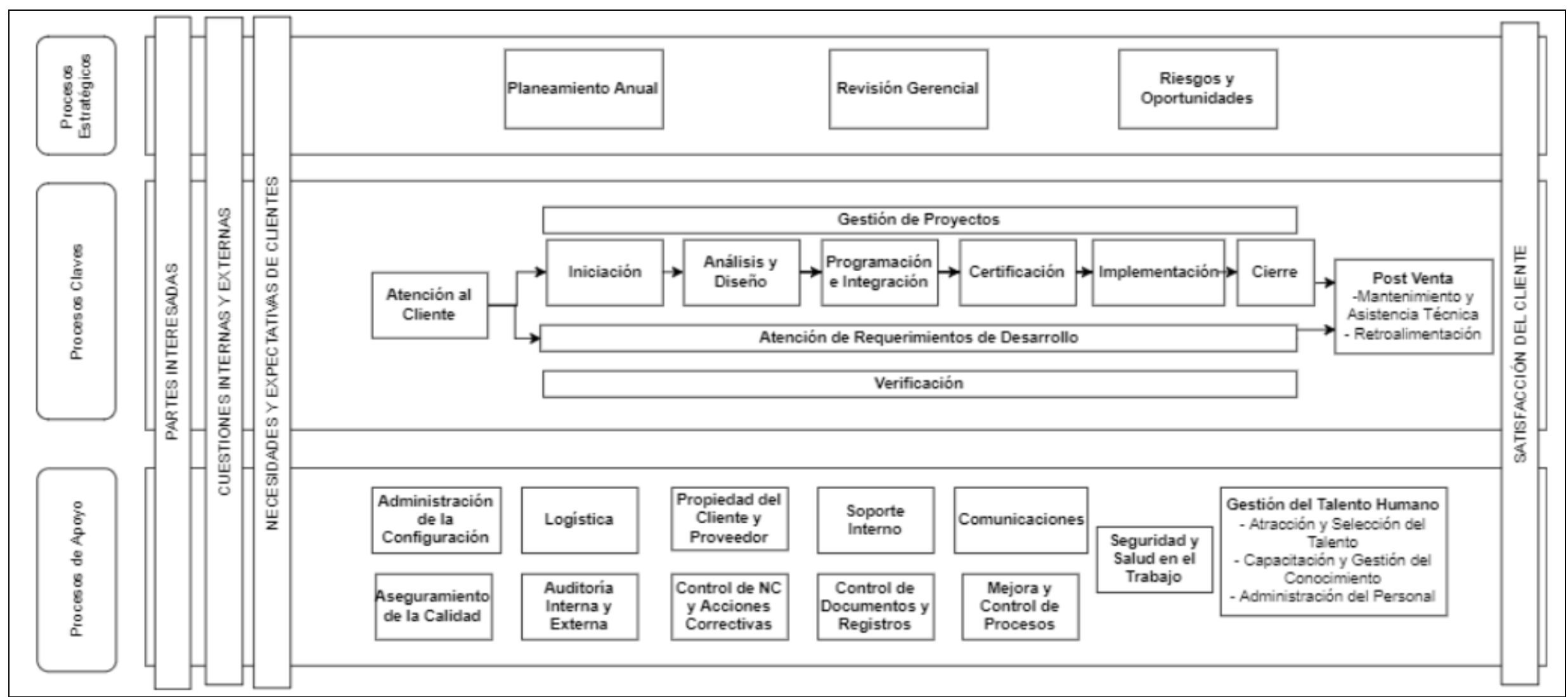

Figura 1. Mapa de procesos de la empresa (ASIS). Adaptado de "Manual de calidad", por El Objeto de Estudio, 2017. 


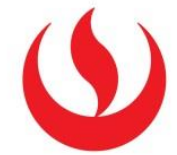

Tabla 2

Objetivos de los Procesos de Negocios

\begin{tabular}{|c|c|c|}
\hline $\mathbf{N}^{\mathbf{o}}$ & Procesos Claves & OBJETIVO DEL PROCESO \\
\hline 1 & Atención al Cliente & $\begin{array}{l}\text { Mantener una retroalimentación continua de las necesidades de los clientes: En } \\
\text { este proceso se elaboran las encuestas de satisfacción global y de proyectos, la } \\
\text { canalización de quejas y la atención de propuestas comerciales. } \\
\text { Recolectar requerimientos y necesidades de los clientes y elaborar propuestas } \\
\text { comerciales que le den frente. }\end{array}$ \\
\hline 2 & $\begin{array}{l}\text { Gestión } \\
\text { Proyectos }\end{array}$ & 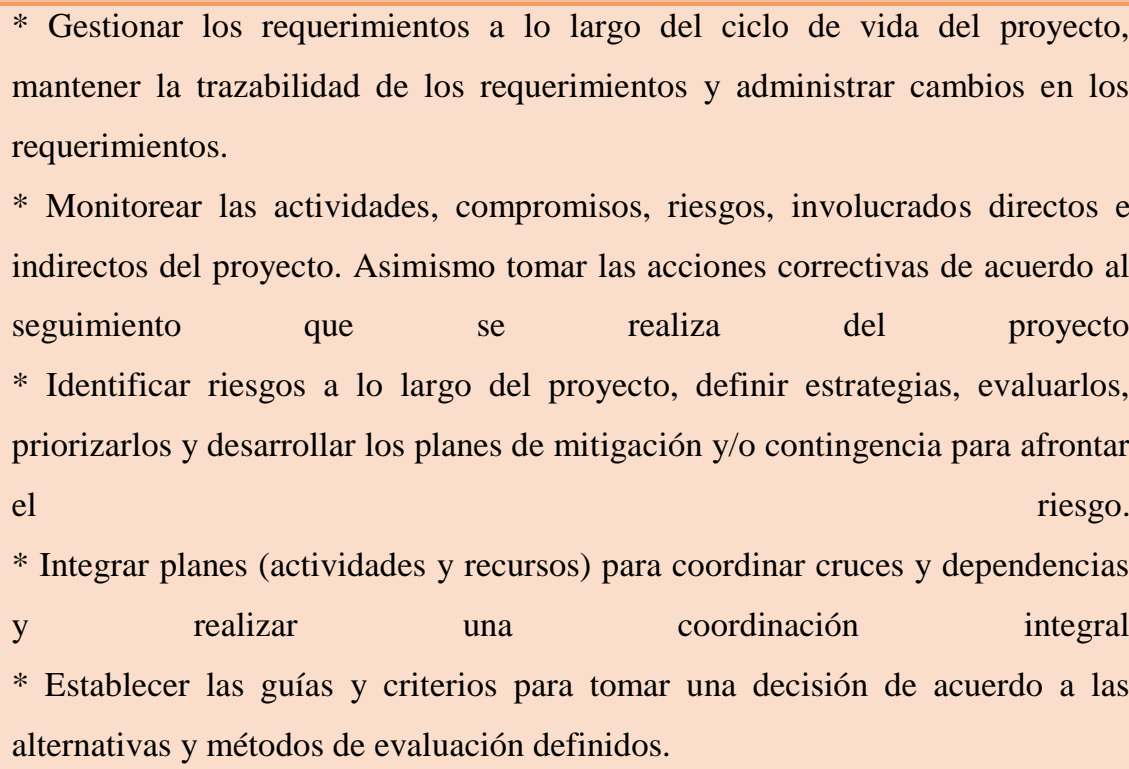 \\
\hline 3 & Iniciación & 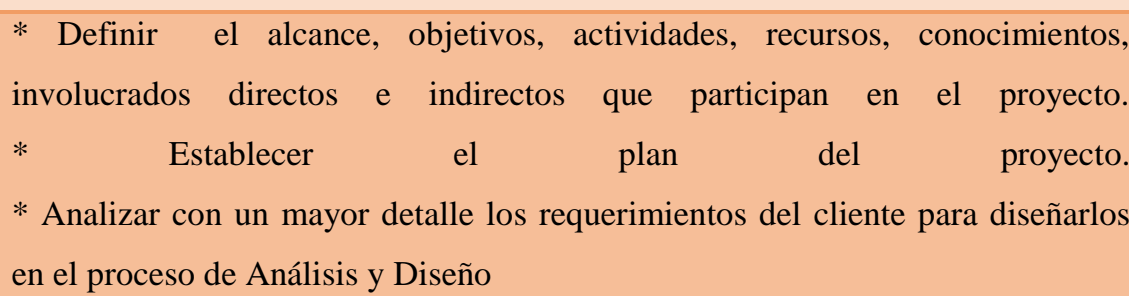 \\
\hline 4 & Análisis y Diseño & $\begin{array}{l}\text { * Elaborar el diseño en función de los requerimientos detallados en Iniciación } \\
\text { para establecer el esquema operacional, la funcionalidad requerida, las } \\
\text { interfaces } \\
\text { * Decesarias, } \\
\text { funcionalidad requerida, en base a las cuales se tome una decisión, diseñar el } \\
\text { producto y sus componentes, diseñar las interfaces, hacer, utilizar, reusar o } \\
\text { comprar los componentes para el producto. }\end{array}$ \\
\hline 5 & $\begin{array}{l}\text { Programación } \\
\text { Integración }\end{array}$ & $\begin{array}{l}\text { * Implementar el diseño, elaborar la documentación necesaria, construcción e } \\
\text { integrar los diferentes componentes del producto, además de validar esta } \\
\text { integración. } \\
\text { * Realizar la preparación y ejecución de las pruebas del producto elaborado }\end{array}$ \\
\hline
\end{tabular}




\section{()}

\begin{tabular}{|c|c|c|}
\hline 6 & Certificación & $\begin{array}{l}\text { Realizar la validación del producto mediante la ejecución de pruebas de } \\
\text { certificación. }\end{array}$ \\
\hline 7 & Implementación & $\begin{array}{l}\text { Instalar el producto desarrollado en los ambientes del cliente para la ejecución } \\
\text { de pruebas finales. }\end{array}$ \\
\hline 8 & Cierre & $\begin{array}{l}\text { Documentar la evaluaciones y otros requisitos para gestionar el cierre del } \\
\text { proyecto en el sistema SARA }\end{array}$ \\
\hline 9 & Verificación & $\begin{array}{l}\text { Realizar la verificación de los entregables del proceso productivo de acuerdo a } \\
\text { criterios establecidos previamente. }\end{array}$ \\
\hline 10 & $\begin{array}{l}\text { Mantenimiento y } \\
\text { Asistencia Técnica }\end{array}$ & $\begin{array}{l}\text { Atender la cartera de clientes con necesidades de mantenimiento y asistencia } \\
\text { técnica, incluye la atención de reportes de problemas y mantenimientos que no } \\
\text { impliquen una modificación o ajuste en el producto. }\end{array}$ \\
\hline 11 & $\begin{array}{l}\text { Atención de } \\
\text { Requerimientos de } \\
\text { Desarrollo, Ajustes } \\
\text { y Modificaciones }\end{array}$ & $\begin{array}{l}\text { Gestionar la cartera de desarrollos, ajustes y modificaciones. Incluye la atención } \\
\text { de Reportes de problemas que implican un ajuste o modificación en el producto. }\end{array}$ \\
\hline $\mathbf{N}^{\mathbf{0}}$ & $\begin{array}{l}\text { Procesos } \\
\text { Estratégicos }\end{array}$ & OBJETIVO DEL PROCESO \\
\hline 12 & Planeamiento Anual & Realizar el plan anual de inversiones de la empresa. \\
\hline 13 & Revisión Gerencial & $\begin{array}{l}\text { Realizar seguimiento a los acuerdos plasmados en las reuniones de revisión } \\
\text { gerencial anuales. }\end{array}$ \\
\hline 14 & $\begin{array}{l}\text { Gestión de Riesgos } \\
\text { y Oportunidades }\end{array}$ & gos y oportunidades, que son de aplicación a la organización. \\
\hline $\mathbf{N}^{\mathbf{o}}$ & Proceso de Apoyo & OBJETIVO DEL PROCESO \\
\hline 15 & Logística & $\begin{array}{l}\text { Gestionar los recursos necesarios para todas las áreas de la empresa poniendo } \\
\text { especial énfasis en los recursos que impactan directamente en la calidad del } \\
\text { producto (servicios externos y otros proveedores) }\end{array}$ \\
\hline 16 & $\begin{array}{l}\text { Gestión de Talento } \\
\text { Humano }\end{array}$ & $\begin{array}{l}\text { Gestionar la capacitación interna y la contratación de personal idóneo para el } \\
\text { desempeño de los procesos. }\end{array}$ \\
\hline 17 & Soporte Interno & Brindar soporte interno a los usuarios de la empresa. \\
\hline 18 & $\begin{array}{l}\text { Administración de } \\
\text { la Configuración }\end{array}$ & $\begin{array}{l}\text { Mantener el orden, control de cambios y trazabilidad de los productos de la } \\
\text { empresa }\end{array}$ \\
\hline 19 & $\begin{array}{l}\text { Propiedad del } \\
\text { Cliente }\end{array}$ & Registrar y administrar la propiedad del cliente utilizada en los proyectos. \\
\hline 20 & $\begin{array}{l}\text { Aseguramiento de } \\
\text { la Calidad }\end{array}$ & Verificación documental de la línea base establecidas para los proyectos \\
\hline 21 & Mejora y Control de & Dar los lineamientos para la gestión de nuevos procesos en la empresa \\
\hline
\end{tabular}




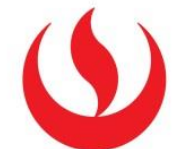

\begin{tabular}{|c|c|c|}
\hline & Procesos & \\
\hline 22 & $\begin{array}{lr}\text { Control de } & \text { No } \\
\text { Conformidades } & \text { y } \\
\text { Acciones } & \\
\text { Correctivas } & \end{array}$ & $\begin{array}{l}\text { Gestionar las no conformidades, potenciales no conformidades, así como las } \\
\text { acciones correctivas que ameriten. }\end{array}$ \\
\hline 23 & $\begin{array}{l}\text { Auditoría Interna y } \\
\text { Externa }\end{array}$ & $\begin{array}{l}\text { Gestionar las auditorías internas y externas de la empresa así como las } \\
\text { observaciones o no conformidades originadas en las mismas. }\end{array}$ \\
\hline 24 & $\begin{array}{lr}\text { Control de } \\
\text { Documentos } \quad y \\
\text { Registros }\end{array}$ & $\begin{array}{l}\text { Establecer las pautas para la elaboración, modificación, publicación y control de } \\
\text { documentos y registros. }\end{array}$ \\
\hline 25 & Comunicaciones & $\begin{array}{l}\text { Gestión de la comunicación efectiva en la empresa, tanto de manera interna, } \\
\text { como hacia las partes interesadas. En este proceso se definirán los canales de } \\
\text { comunicación internos y externos establecidos por la empresa. } \\
\text { Mantener al personal interno/externo informado acerca de los asuntos y } \\
\text { decisiones relativas al funcionamiento del Sistema de Gestión de la Calidad. }\end{array}$ \\
\hline 26 & $\begin{array}{l}\text { Seguridad y Salud } \\
\text { en el Trabajo }\end{array}$ & $\begin{array}{l}\text { Mantener el Sistema de Gestión de Seguridad y Salud en el Trabajo en la } \\
\text { organización y cumplir con la normatividad nacional vigente aplicable en } \\
\text { materia de riesgos laborales. }\end{array}$ \\
\hline
\end{tabular}

Nota: Se ha descrito los objetivos de los procesos claves, estratégicos y de apoyo. Adaptado de "Manual de calidad”, por El Objeto de Estudio, 2017. 
ORGANIGRAMA

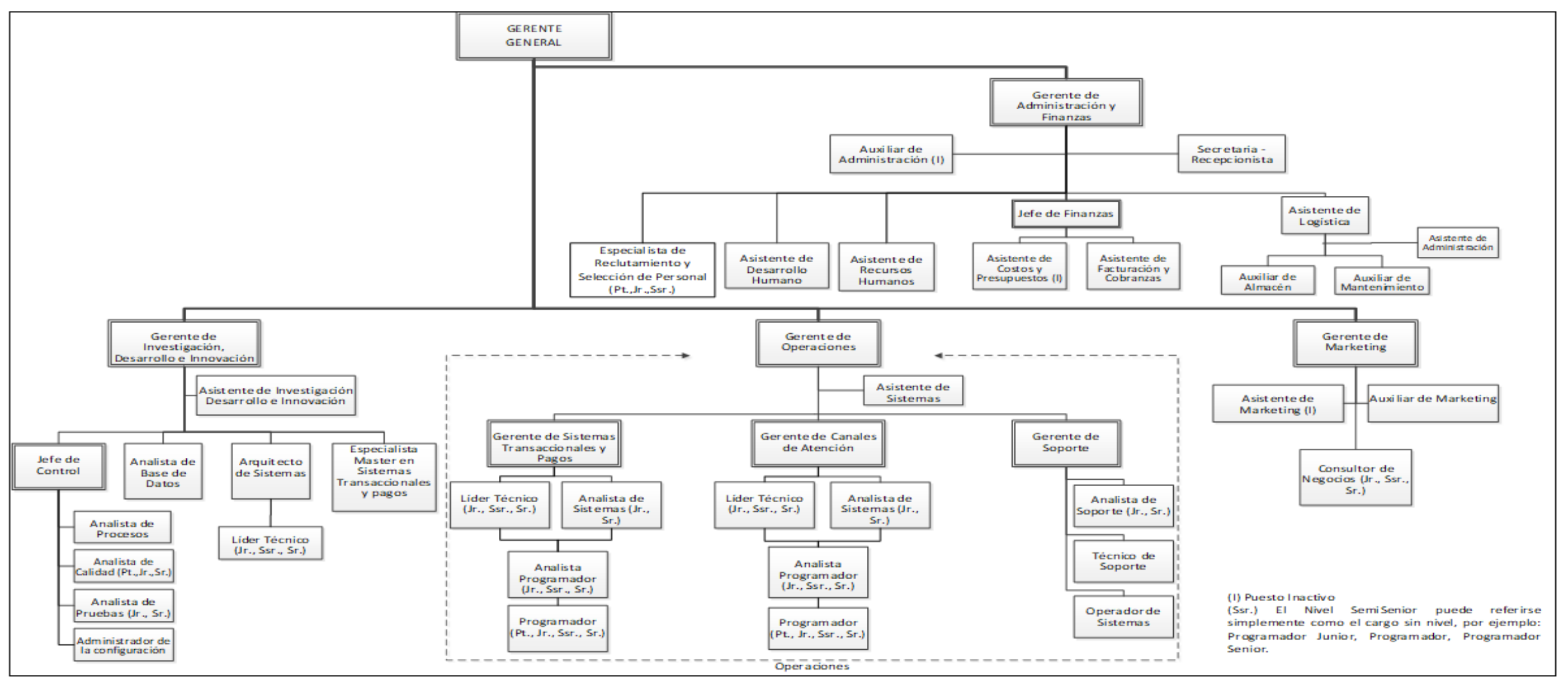

Figura 2. Organigrama de la empresa. Adaptado de "Manual de funciones y perfiles de puestos", por El Objeto de Estudio, 2017. 


\section{(1)}

Tal como indica el Objeto de Estudio (2017), a continuación se describen brevemente los roles indicados en el organigrama:

1. Gerente General: Encargado de planificar, organizar, dirigir y controlar las actividades y procedimientos de su Gerencia, con la finalidad de establecer y difundir dentro de la organización, la visión, misión, valores y estrategias de la empresa, asegurando su crecimiento y rentabilidad en estándares superiores al mercado y el uso de prácticas empresariales de vanguardia.

\section{2. Área de Administración y Finanzas}

2.1. Gerente de Administración y Finanzas: Encargado de planificar, dirigir, controlar y ejecutar los procesos administrativos, financieros y comerciales; con la finalidad de asegurar la administración eficiente de los recursos humanos, financieros, materiales y servicios requeridos, contribuyendo así al cumplimiento del marco legal vigente, de la misión y de los objetivos institucionales.

2.2. Jefe de Finanzas: Encargado de asegurar y gestionar los recursos financieros que permitan que la empresa pueda cubrir sus necesidades de operación así como el financiamiento de sus planes de inversión, basándose en los presupuestos de la empresa, gestionando los recursos con financiamiento externo, distribuyendo los recursos según planificación con la finalidad de garantizar el financiamiento de los planes y que todos los pagos se realicen de acuerdo a la normas.

2.3. Auxiliar de Administración: Encargado de apoyar en el desarrollo de actividades de gestión del personal y administrativos de la empresa, con el fin de contribuir al desarrollo de los planes de desarrollo y administración del mismo.

2.4. Secretaria - Recepcionista: Encargado de brindar atención y canalizar de forma oportuna y ágil a los requerimientos y/o consultas de los clientes externos e internos, u otras entidades, con el fin de contribuir a la fluidez de la comunicación y operaciones de la empresa. 


\section{(1)}

2.5. Asistente de Facturación y Cobranzas: Encargado de controlar y velar para que se cumpla con los procesos de facturación y cobranzas, para poder brindar información relevante a la alta gerencia y a las áreas de negocio. Asimismo controlar la caja chica para hacer un seguimiento de los gastos en los distintos centros de costos.

2.6. Asistente de Costos y Presupuestos: Encargado de controlar el presupuesto de inversiones y gastos operativos así como la rentabilidad de los proyectos de la empresa, a fin de brindar información que permita una oportuna toma de decisiones.

2.7. Asistente de Recursos Humanos: Encargado de planificar, coordinar y ejecutar acciones que contribuyan a la administración del personal de la empresa, con el fin de cumplir con las normas y políticas de la Organización así como con la legislación laboral vigente.

2.8. Asistente de Desarrollo Humano: Encargado de planificar y ejecutar las actividades de Desarrollo Humano en la empresa, con el fin de asegurar a ésta, las personas competentes, motivadas y productivas que requiere para el logro de los objetivos planteados para la misma.

2.9. Especialista de Reclutamiento y Selección de Personal: Encargado de planificar y ejecutar las actividades de reclutamiento y selección de la empresa, con el fin de cubrir la dotación de recurso humano competente e idóneo, acorde a las necesidades organizacionales.

2.10. Asistente de Logística: Encargado de gestionar el Proceso de Adquisiciones de Bienes y Servicios de la empresa así como planificar y supervisar el plan de mantenimiento de la misma, a fin de garantizar la atención de las necesidades del cliente interno y mantener en óptimas condiciones las instalaciones de la empresa.

2.11. Asistente de Administración: Encargado de brindar soporte al área de Administración y Finanzas con el objetivo de contribuir a la fluidez de las operaciones del área y al cumplimiento de los objetivos. 


\section{(1)}

2.12. Auxiliar de Almacén: Encargado de administrar el almacén y bienes de la empresa, a fin de lograr su adecuada conservación y salvaguardar los intereses de la empresa.

2.13. Auxiliar de Mantenimiento: Encargado de atender los diversos requerimientos de servicios generales de la empresa, a fin de contribuir con el desarrollo de las operaciones de la misma.

\section{Gerencia de Marketing}

3.1. Gerente de Marketing: Encargado de Planificar, organizar, dirigir, y controlar las actividades y procedimientos de su Gerencia, con la finalidad de asegurar la administración eficiente de los recursos humanos, financieros, materiales y servicios requeridos para realizar en forma óptima las funciones de Mercadeo, tanto a nivel estratégico como operacional; contribuyendo así al cumplimiento de la misión y objetivos institucionales.

3.2. Consultor de Negocios: Encargado de planificar y controlar la gestión de ventas a nivel nacional e internacional, a fin de garantizar la aplicación de las políticas de atención y servicio al cliente y el cumplimiento de plan de ventas. Así mismo, facilitar soluciones transaccionales basadas en software especializado, de acuerdo a la necesidad de cada empresa, a fin de incrementar la participación y cuotas de mercado a nivel nacional e internacional.

3.3. Asistente de Marketing: Encargado de apoyar a la Gerencia de Marketing y al personal del área en el proceso de atención al cliente, en las diferentes necesidades del área, a fin de contribuir al logro de las metas trazadas.

\section{Gerencia de Investigación, Desarrollo e Innovación}

4.1. Gerente de Investigación, Desarrollo e Innovación: Encargado de Planificar, organizar, dirigir, y controlar las actividades y procedimientos de su Gerencia, con la finalidad de asegurar la administración eficiente de los recursos humanos, financieros, materiales y servicios requeridos para realizar permanentemente la labor 


\section{(1)}

de investigación y evaluación del avance tecnológico y su aplicación en los sistemas y productos de la empresa, así como la de velar por el funcionamiento de los Sistemas de Gestión de Calidad; contribuyendo así al cumplimiento de la misión y objetivos institucionales.

4.2. Jefe de Control: Encargado de Planificar, organizar, dirigir y controlar las actividades de control de calidad de los procesos y productos de la organización, con el objetivo de lograr la satisfacción de los clientes y el cumplimiento del Sistema de Gestión de la Calidad (SGC).

4.3. Analista de Procesos: Encargado de analizar, generar, desarrollar e implementar procedimientos, métodos de trabajo y normas en los procesos de la empresa, con el fin de optimizarlos y lograr así la administración eficaz de los recursos de la organización.

4.4. Analista de Calidad: Encargado de analizar, monitorear y controlar el cumplimiento de los procesos descritos por la organización, a fin de buscar la mejora continua de nuestros procesos y el cumplimiento de los requisitos del sistema de Gestión de Calidad de la organización.

4.5. Analista de Pruebas: Encargado de planificar, diseñar y ejecutar los casos de pruebas, a fin de garantizar la calidad del producto o proyecto y el cumplimiento de los requerimientos.

4.6. Administrador de la Configuración: Encargado de administrar los ítems de la configuración generados en las diferentes etapas de un proyecto o ATR y supervisar la realización del proceso de la Administración de la Configuración, a fin de garantizar el resguardo adecuado de la información y el cumplimiento de los requisitos el Sistema de Gestión de Calidad de la empresa.

4.7. Arquitecto de Sistemas: Encargado de definir, mantener, revisar y mejorar la arquitectura de los sistemas (sistemas, sub-sistemas, productos, servicios, procesos, etc.) considerando los intereses/requerimientos de la empresa y de sus clientes. 
4.8. Líder Técnico: Encargado de velar por el cumplimiento técnico de los productos, sistemas y servicios de la empresa, considerando los intereses / requerimientos técnicos tales como arquitectura, buenas prácticas y estándares de la empresa y de sus clientes.

4.9. Especialista Master en Sistemas Transaccionales y Pagos: Encargado de investigar, revisar y asesorar a las diferentes áreas sobre Sistemas de Seguridad y Sistemas de Transferencia Electrónica.

4.10. Asistente de Investigación, Desarrollo e Innovación: Encargado de brindar apoyo en las actividades administrativas inherentes a la Gerencia General y la Gerencia de Investigación y Desarrollo, garantizando la realización y desarrollo de las tareas asignadas que conlleven un grado de responsabilidad y confiabilidad, para lograr los objetivos y alcanzar las metas trazadas por la Organización.

\section{Gerencia de Operaciones}

5.1. Gerente de Operaciones: Encargado de planificar, organizar, dirigir, y controlar las actividades y procedimientos de su Gerencia, con la finalidad de asegurar la administración eficiente de los recursos humanos, financieros, materiales y servicios requeridos para el correcto desarrollo de los proyectos y productos, así como brindar un óptimo mantenimiento y asistencia técnica a los clientes; contribuyendo así al cumplimiento de la misión y objetivos institucionales.

5.2. Gerente de Canales de Atención: Encargado de planificar, organizar, dirigir y controlar las actividades del área de Canales de Atención, con el objetivo de lograr la satisfacción de los clientes mediante una adecuada gestión de los proyectos y productos asignados así como del cumplimiento del Sistema de Gestión de la Calidad (SGC).

5.3. Gerente de Sistemas de Pago: Encargado de planificar, organizar, dirigir y controlar las actividades del área de Sistemas de Pago, con el objetivo de lograr la satisfacción de los clientes mediante una adecuada gestión de los proyectos y productos asignados así como del cumplimiento del Sistema de Gestión de la Calidad (SGC). 


\section{(1)}

5.4. Analista de Sistemas: Encargado de analizar, diseñar, desarrollar e implementar productos y/o adecuaciones de los mismos cumpliendo las normas y procedimientos establecidos, a fin de satisfacer los requerimientos de los clientes e internos, garantizando la calidad del producto y la satisfacción del cliente.

5.5. Analista Programador: Encargado de analizar y describir los requerimientos para elaborar especificaciones funcionales y técnicas, así como ejecutar las actividades para convertir dichas especificaciones en un código de software, a fin de garantizar la calidad del producto y la satisfacción del cliente.

5.6. Programador: Encargado de ejecutar las tareas de programación e implementación de los proyectos y atenciones de requerimiento asignados aplicando los estándares de programación, las normas y procedimientos de la Institución para entregar un producto de calidad que cumpla con los requerimientos del cliente.

5.7. Asistente de Sistemas: Encargado de colaborar con las tareas administrativas y de gestión de las Gerencias de Producto, a fin de contribuir con el cumplimiento de los cronogramas y objetivos trazados para dichas áreas.

5.8. Gerente de Soporte: Encargado de brindar solución a los problemas que reporten los clientes y/o usuarios del Objeto de Estudio, de la manera más eficiente, en los tiempos acordados, proveyendo un soporte especializado y ágil con conocimiento y herramientas de TI, con el objetivo de asegurar un nivel de satisfacción elevado en los mismos.

5.9. Analista de Soporte: Encargado de realizar el análisis y corrección de los reportes de problemas reportados por los clientes, así como atender sus consultas en las implementaciones de producción, a fin de contribuir a que funcionen correctamente según lo definido en la documentación correspondiente.

5.10. Técnico de Soporte: Encargado de velar porque la infraestructura tecnológica de la empresa se encuentre operativa con el fin de permitir el desarrollo de las operaciones de la empresa. 


\section{(1)}

5.11. Operador de Sistemas: Encargado de mantener la operatividad y continuidad del servicio "Sistema de procesamiento transaccional", a fin de garantizar en todo momento la disponibilidad del servicio frente a los clientes y autorizadores, en base al manejo de procedimientos y herramientas de gestión propias del sistema.

\section{ALCANCE DEL PROYECTO}

Dentro de los procesos estratégicos el Objeto de Estudio cuenta con los siguientes: Planeamiento Anual, Revisión Gerencial y Riesgos y Oportunidades, los cuales no están directamente relacionados a la cadena de valor de la empresa y son exclusivamente administrados por la alta gerencia. Debido a ello, no se podría alinear y aprovechar las ventajas de una arquitectura empresarial ya que estos procesos no son del negocio ni del área de TI.

Como proceso seleccionado para analizar es el de Gestión de Proyectos ya que al ser una empresa dedicada al rubro de tecnología es decir desarrollo, gestión y consultoría de software, este proceso es uno de los más importantes en su cadena de valor porque dirige y puede otorgar el éxito de un proyecto interno o externo. Además, contribuyó el análisis de los problemas que presenta actualmente el proceso, los cuales se presentarán más adelante, tanto a nivel de negocio y de las herramientas de sistemas de información que le dan soporte.

Por consecuente, el presente proyecto tiene como alcance el análisis y la realización de una propuesta de arquitectura empresarial, la cual nos permitirá alinear las decisiones de la organización con el área de TI, a fin de cumplir con los objetivos estratégicos propuestos.

El análisis a desarrollar considerará:

- La evaluación de requerimientos de negocio y TI, y su alineamiento con los objetivos estratégicos de la empresa bajo el framework propuesto por The Open Group llamado TOGAF.

- Utilización del framework SCRUM para el desarrollo de un proyecto como propuesta de mejora tecnológica para la organización. 


\section{(1)}

\section{OBJETIVOS DEL PROYECTO}

\section{OBJETIVO GENERAL.}

Elaborar una propuesta de arquitectura empresarial enfocada en la mejora y optimización del proceso de Gestión de Proyectos y sus herramientas del Objeto de Estudio para un mejor desempeño de la organización.

\section{OBJETIVOS ESPECÍFICOS.}

- Realizar el estudio de la situación actual del proceso de Gestión de Proyectos enfocado a los cuatro dominios de la arquitectura empresarial basado en el framework TOGAF.

- Realizar el estudio de la situación deseada del proceso de Gestión de Proyectos enfocado a los cuatro dominios de la arquitectura empresarial basado en el framework TOGAF.

- Identificar los proyectos que cubrirán las brechas detectadas entre la situación actual y deseada del proceso.

- Proponer un software como herramienta de soporte al proceso de gestión, que reemplace a las herramientas existentes.

- Proponer la actualización del procedimiento de Gestión de Proyectos en base a la nueva herramienta propuesta.

- Proponer la aplicación del framework Scrum para el desarrollo de software en un proyecto del plan de implementación y migración de Arquitectura Empresarial. 


\section{(1)}

\section{BENEFICIOS DEL PROYECTO}

\section{BENEFICIOS TANGIBLES}

- Reducción de al menos 9\% en la actualización y procesamiento de cronogramas en el proceso de Gestión de Proyectos. (Ver anexo 2)

- Reducción del $100 \%$ de no conformidades que actualmente tiene el proceso por incumplimiento del procesamiento de cronogramas y actualización de matriz de trazabilidad y lista de riesgos. (Ver anexo 3)

\section{BENEFICIOS INTANGIBLES.}

- Conocimiento integral por parte de la organización sobre los posibles cambios del proceso de Gestión de Proyectos.

- Contar con la información centralizada de gestión de proyectos en un solo aplicativo.

- Tener información confiable y en tiempo real sobre los entregables de gestión de proyectos. 
CAPÍTULO 2: MARCO TEÓRICO 


\section{(1)}

\section{MARCO TEÓRICO}

\section{Arquitectura Empresarial}

Zachman (1987), planteó un marco teórico para la elaboración de una arquitectura de sistemas de información, en la cual se definen y controlan todos los componentes del sistema. Para ello realizó investigaciones sobre alineamientos estratégicos y arquitecturas de sistemas de información, organizando el concepto de arquitectura de sistemas en base a las definiciones de software, datos e infraestructura con los procesos de negocio.

El marco teórico de Zachman para el desarrollo de arquitecturas empresariales, distribuye perfectamente la arquitectura de negocio, procesos de negocio y objetivos empresariales, con las arquitecturas de aplicaciones, arquitectura de datos y arquitectura tecnológica de la empresa; cada elemento de cada arquitectura se encuentra correctamente enlazado logrando una sincronía total y con ello una excelente gestión de negocio.

Según Arango-Serna, Londoño-Salazar \& Branch-Bedoya (2015) indican que la Arquitectura Empresarial (AE) se concentra en apoyar los siguientes aspectos:

- Acompañar el desarrollo y la ejecución de la estrategia de negocio en su proceso de transformación contínuo.

- Mejorar de manera progresiva los procesos y las capacidades de negocio.

- Operacionalizar en la práctica los cambios que muestra la empresa, y que influyen toda la estructura operativa.

- Apoyar la orientación tecnológica alineado con las estrategias de negocio y examinar capacidades de innovación a partir de los recursos y capacidades existentes 


\section{(4)}

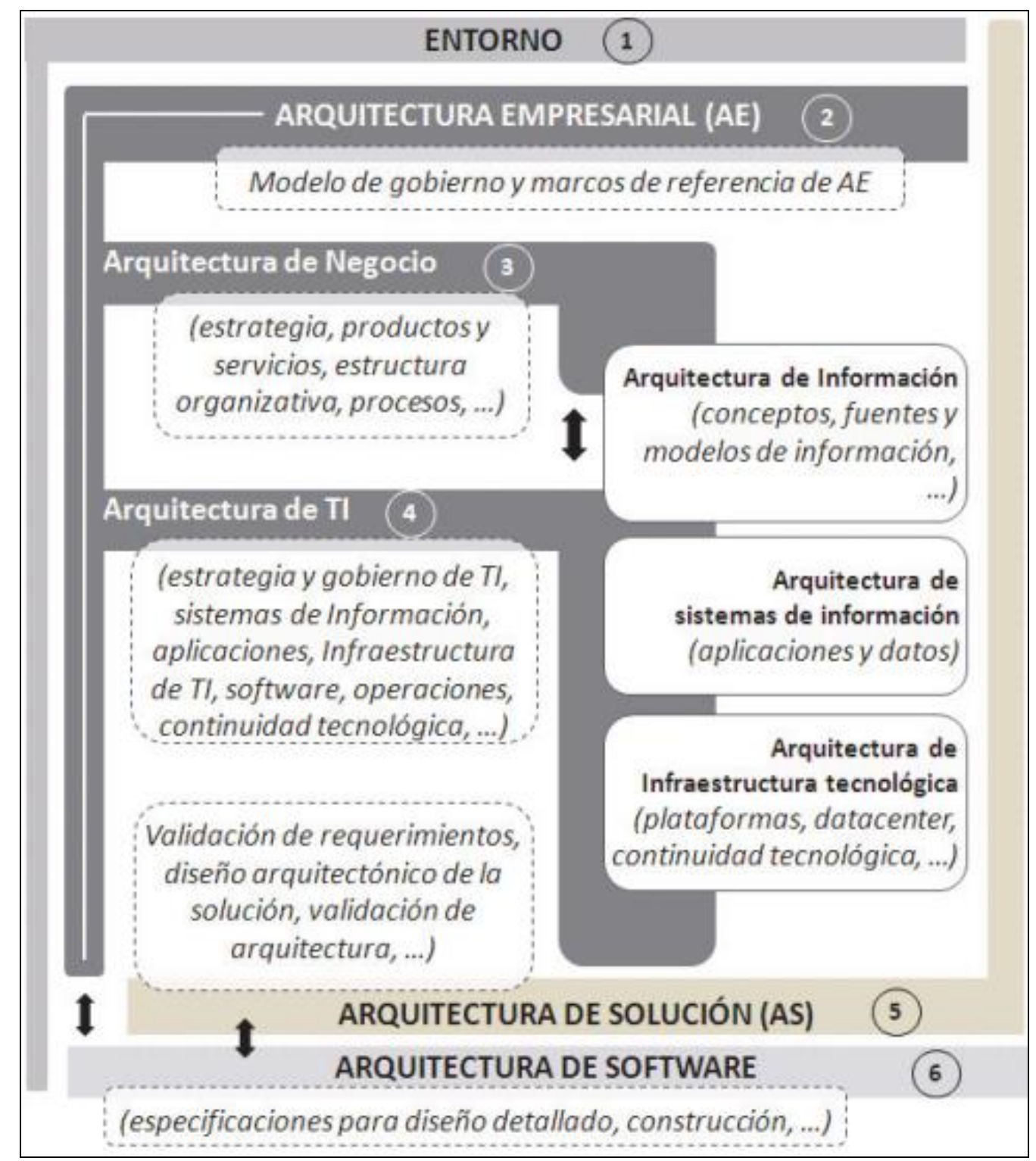

Figura 3. Entorno y enfoques de Arquitectura Empresarial y de TI. Adaptado de "Enfoque de arquitectura de solución, mecanismo para reducir la brecha entre la arquitectura empresarial y la implementación de soluciones tecnológicas", por Revista de Ingenería Dyna, 2015.

\section{Vistas de la arquitectura empresarial}

La $\mathrm{AE}^{9}$ tiene diferentes vistas o componentes los cuales sirven como herramientas para el soporte de las operaciones del negocio. Según Arango, Lodoño \& Zapata (2010), existen cuatro perspectivas de la Arquitectura Empresarial: Arquitectura de Negocio, Arquitectura de Información, Arquitectura de Aplicaciones y Arquitectura Tecnológica.

\footnotetext{
${ }^{9}$ Para ver el significado de “AE” ir a la sección SIGLARIO del documento.
} 


\section{(1)}

\section{- Arquitectura de negocio}

Es la arquitectura responsable de la descripción de la estructura organizacional, de los procesos de negocio, los sistemas de planeación y control, los mecanismos de gobierno y administración de políticas y procedimientos en el entorno empresarial.

Aparte de ello, la arquitectura de negocio se lleva a cabo en base al plan estratégico de la empresa, los lineamientos corporativos, los indicadores de gestión, y se nutre de la misión, la visión, las estrategias y los objetivos corporativos. Además, en esta vista se definen los procesos empresariales y su relación con clientes y usuarios y se crea un modelo de arquitectura en el que se superan las exigencias de estos.

Finalmente, la arquitectura de negocio llega al punto de mejorar los procesos alineados con las estrategias de negocio en el contexto corporativo.

\section{- Arquitectura de información}

Es la arquitectura responsable de la descripción de los activos lógicos y físicos, de los datos como un activo de la empresa y la administración de los recursos de información. La finalidad de esta vista es exponer como los recursos de información están siendo administrados, utilizados y compartidos por la empresa. Se emplea con el objetivo de asegurar que se cuente con repositorios y fuentes únicas de información precisa y oportuna que la organización necesita para dar frente a los diferentes procesos y destinarla para diferente propósitos.

\section{- Arquitectura de aplicaciones}

Es la arquitectura responsable de añadir soluciones aplicativas que afirmen el negocio basadas en las capacidades funcionales y en las estrategias de tecnología definidas. Aparte de ello, define cuáles son las aplicaciones importantes para la organización y valida los requerimientos necesarios para gestionar los datos y presentar la información.

\section{- Arquitectura tecnológica}

Es la arquitectura responsable de establecer el marco tecnológico de las plataformas computacionales y bases de datos que deben soportar las distintas soluciones del 


\section{(1)}

negocio, además los mecanismos de almacenamiento de datos e información, las redes de datos, los centros de procesamiento de datos y los servicios integrados de tecnología.

\section{Beneficios de la arquitectura empresarial}

Según Arango, Lodoño \& Zapata (2010), las ventajas que brinda la Arquitectura Empresarial a cada organización son los mencionados a continuación:

- La AE conlleva a que se pueda definir una visión clara sobre los objetivos, las metas, y líneas de negocio en la empresa.

- Permite el reconocimiento del estado actual de la empresa y la detalla como una estructura coherente y articulada en todos sus componentes.

- Actúa como impulso integrador entre aspectos de planificación del negocio, de operación del negocio y aspectos tecnológicos.

- Permite estar al tanto de forma real, medible y detallada, la brecha que está entre el estado actual de los procesos del negocio y la tecnología que los soporta, respecto al estado deseado que requiere la dirección estratégica.

- Permite unificar, mejorar y/o eliminar procesos y tecnologías redundantes, disminuyendo costos operacionales.

- Actúa como una plataforma corporativa que afirma y prepara a la empresa para afrontar de manera fácil y oportuna cambios en el mercado, retos de crecimiento y respuesta a la competencia, entre otros.

- Mejora el desempeño y productividad de la empresa.

\section{¿Cómo se desarrolla una Arquitectura Empresarial?}

Según Molano (2015), los marcos de trabajos se enfocan en agilizar y simplificar la definición y desarrollo de la arquitectura, aseverando un cubrimiento más completo de la solución y certifican que la arquitectura selecciona permita el crecimiento futuro en respuesta a las necesidades del negocio. Existen cuatro marcos de trabajo de alto reconocimiento para desarrollar la AE: 


\section{(1)}

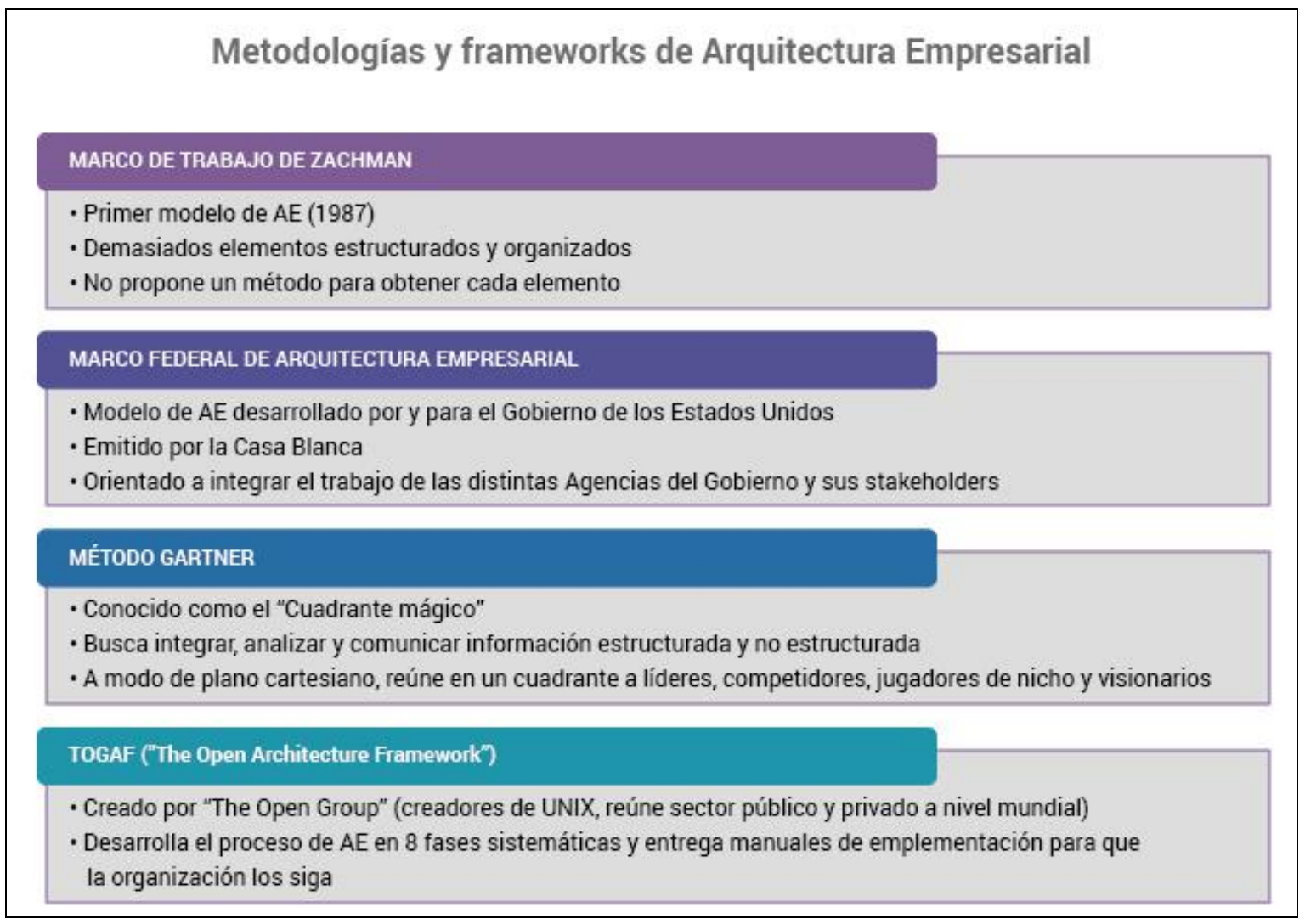

Figura 4. Metodologías y frameworks de arquitectura empresarial. Adaptado de “¿Qué es Arquitectura Empresarial?", por Adriana Molano, 2015.

\section{TOGAF}

Según The Open Group (1999 - 2018), TOGAF es un marco de trabajo que puede ser utilizado libremente por cualquier empresa que requiera desarrollar una arquitectura empresarial.

El origen de TOGAF se llevó a cabo en base al Marco de Arquitectura Técnica para la Gestión de la Información (TAFIM), desarrollado por el Departamento de Defensa de los Estados Unidos (DoD). El Departamento de Defensa otorgó a The Open Group permiso explícito para crear la versión 1 del estándar TOGAF basándose en el TAFIM. A partir de esa sólida base se han desarrollado versiones sucesivas de TOGAF y publicado cada una en el sitio web de The Open Group. 


\section{$(1)$}

TOGAF abarca el desarrollo de cuatro dominios de arquitecturas que vienen a ser como subconjuntos de una arquitectura empresarial, los cuales son soportados por TOGAF y se detallan:

La arquitectura de negocios: define la estrategia comercial, el gobierno, la organización y los procesos clave de negocio.

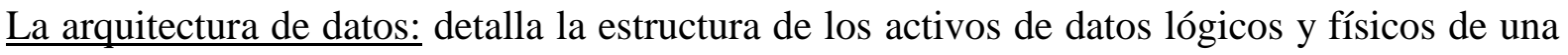
empresa y los recursos de gestión de datos.

La arquitectura de aplicaciones: proporciona un modelo para las aplicaciones individuales que se implementarán, sus interacciones y sus relaciones con los procesos empresariales centrales de la organización.

La arquitectura de tecnología: detalla las capacidades lógicas de software y hardware que se requieren para admitir la implementación de servicios empresariales, de datos y de aplicaciones; esto incluye infraestructura de TI, middleware, redes, comunicaciones, procesamiento, estándares, etc.

\section{Método de Desarrollo de Arquitectura}

El Método de Desarrollo de Arquitectura (ADM) proporciona un proceso validado y repetible para el desarrollo de arquitecturas. El ADM incluye el establecimiento de un marco de arquitectura, el desarrollo de contenido de arquitectura, la transición y el gobierno de la realización de arquitecturas. Todas estas actividades se llevan a cabo dentro de un ciclo iterativo de definición y realización continua de arquitecturas que permite a las organizaciones transformar sus empresas de forma controlada en respuesta a los objetivos y oportunidades comerciales. Las fases dentro del ADM son las siguientes:

Fase Preliminar: Esta fase sirve para determinar la capacidad de arquitectura deseada por la organización. Con ella podremos:

- Analizar el contexto organizacional

- Determinar e identificar el alcance de los elementos organizacionales

- Identificar los métodos, marcos y procesos establecidos

- Determinar el objetivo de madurez de la capacidad

- Establecer el modelo organizacional para la arquitectura empresarial 
- Definir el detalle del proceso y los recursos para la arquitectura

- Implementar herramientas que soporten la capacidad de la arquitectura

- Definir los principios de arquitectura

\section{Fase A: Visión de la Arquitectura}

- Desarrollar una visión de alto nivel de las capacidades y el valor comercial que se otorgaran como resultado de la arquitectura empresarial propuesta.

- Obtener la aprobación para una Declaración de Trabajo de Arquitectura que especifica un programa de trabajos para desarrollar y desplegar la arquitectura descrita en la Visión de Arquitectura.

\section{Fase B: Arquitectura de Negocio:}

- Describir como la organización necesita trabajar para alcanzar los objetivos de negocio y responda a los factores estratégicos establecidos en la Visión de Arquitectura, de una manera que aborde la Declaración de Trabajo de Arquitectura y las preocupaciones de los interesados.

- Identificar los elementos de la hoja de ruta de la arquitectura en base a las diferencias entre la línea base y las arquitecturas organizacionales objetivo.

\section{Fase C: Arquitectura de Sistemas de Información:}

- Detallar como la arquitectura de sistemas de información de la empresa permitirá la arquitectura de negocios y la visión de la arquitectura, de tal forma que aborde sobre la declaración del trabajo de arquitectura y las preocupaciones de los interesados.

- Identificar los elementos de la hoja de ruta de la arquitectura en base a las brechas entre la línea base y la de destino (datos y aplicaciones).

\section{Fase D: Arquitectura Tecnológica:}

- Habilitar la visión de la arquitectura, objetivo del negocio, datos, y bloques de creación de aplicaciones que se darán a través de componentes de tecnología y servicios, de una manera que aborde el trabajo de arquitectura y las preocupaciones de los interesados. 


\section{$(1$}

- Identificar los elementos de la hoja de ruta de la arquitectura en base a las brechas entre la línea base y la de destino (tecnología).

\section{Fase E: Oportunidades y Soluciones:}

- Generar la hoja de ruta de la arquitectura basada en el análisis de brechas y las arquitecturas de las fases B, C y D.

- Determinar si se requiere un enfoque incremental, identificar las arquitecturas de transacción que brindaran un valor comercial.

- Definir la construcción de la solución general para finalizar la arquitectura basada en bloques de construcción de arquitectura.

\section{Fase F: Planeación de Migraciones:}

- Finalizar la hora de ruta de la arquitectura y el plan de implementación y migración de apoyo.

- Asegurar que el plan de implementación y migración haya sido coordinado con el enfoque de la organización para poder administrar e implementar el cambio en la cartera de cambios globales en la organización.

- Validar que las partes interesadas entiendan el valor comercial y el costo de los trabajos y arquitecturas en transacción.

\section{Fase G: Implementación de la Gobernanza:}

- Garantizar la conformidad con la arquitectura mediante los proyectos de implementación.

- Ejecutar las funciones de arquitectura adecuada para la solución y cualquier arquitectura impulsada por la implementación.

\section{Fase H: Gestión de la arquitectura de cambio}

- Validar que se mantiene el ciclo de vida de la arquitectura.

- Validar que se ejecute el marco de la arquitectura.

- Validar que la capacidad de arquitectura empresarial cumpla con los requisitos actuales. 


\section{()}

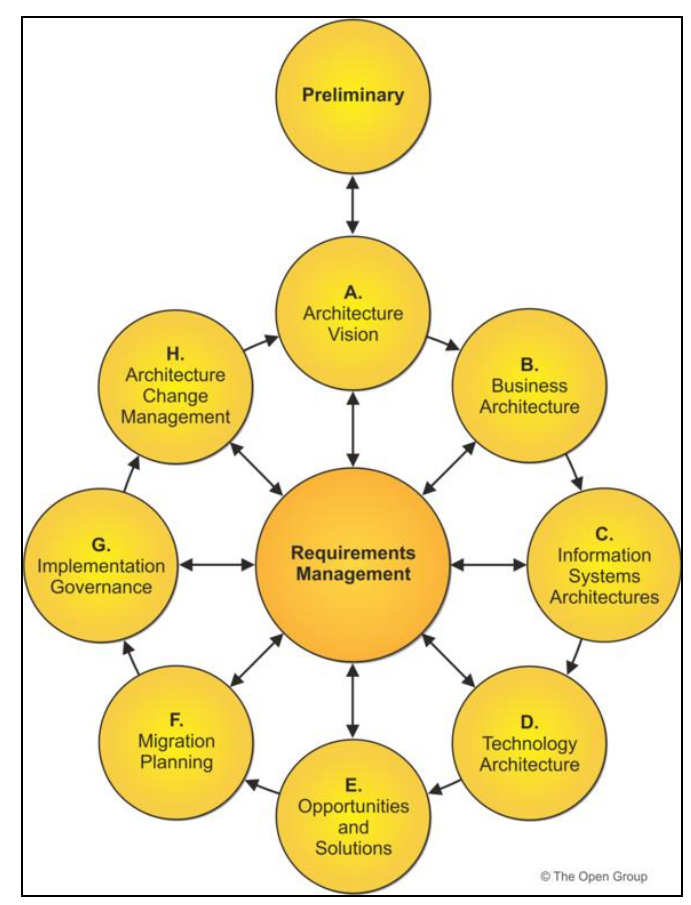

Figura 5. Ciclo de desarrollo de arquitectura. Adaptado de "Part II - Architecture Development Method", por The Open Group, 1999 - 2018.

\section{CASO DE ÉXITO}

Según lo indicado por Craftware Consultores LTDA (2014), trabajó en un proyecto exitoso con la empresa Redbanc, el cual publican como un Caso de Éxito de Arquitectura Empresarial y que consistió en la creación de un Repositorio de Arquitectura, en el que se modela la empresa REDBANC, la cual integra armónicamente sus diversas vistas: Negocio, Datos, Aplicaciones y Tecnologías, junto con sus interrelaciones para fundar los cimientos sobre los que se empujan la gestión y el desarrollo continuo de la Arquitectura Empresarial.

\section{Situación Actual:}

La empresa para identificar de manera rápida cualquier impacto acude al conocimiento de las personas de mayor experiencia en la organización. Debido a que la documentación existente no siempre permite hacer el cruce entre los dominios relacionados a un cambio particular. Actualmente es un riesgo para la organización no contar con una manera de consolidar y sistematizar el conocimiento existente, lo que conlleva el riesgo de hacer análisis que se omitan elementos relevantes de algún dominio, o que carezcan de una visión integral del real impacto de introducir un cambio a la Arquitectura de la organización. 


\section{(1)}

\section{Estrategias implementadas:}

Para la ejecución particular de este proyecto se siguieron los siguientes principios y estrategias:

- Se eligió trabajar con UML como notación base. Debido a ello, se definió un subconjunto de sus elementos sobre el cual se basó la construcción del Modelo. Este subconjunto se personalizó de acuerdo a las necesidades particulares de Redbanc con el uso de estereotipos.

- Al realizar la personalización con el uso de estereotipos se buscó que la representación fuera lo más parecido posible a la realidad existente, respetando el nombre que se le dan a los elementos en el contexto interno de Redbanc.

- La AE se colocó en un modelo único construido con la herramienta Enterprise Architect, sobre dicho modelo se puede efectuar el análisis de impacto tanto de una manera visual-manual como automatizada.

- Redbanc a partir de este proyecto ha definido una política clara de revisión periódica y de actualización del modelo.

- Mediante el uso de la funcionalidad de generación automática de documentación que entrega la herramienta Enterprise Architect, se obtienen informes de la evaluación de impacto debido a cambios en servicios y aplicaciones.

- Sobre el modelo integral se puede trabajar colaborativamente, permitiendo la creación de usuarios de las distintas áreas ser dueños de su parte de la AE.

- Se construyó un validador escrito en JScript, que permite velar por el correcto uso de las notaciones y estándares de modo de asegurar el correcto uso del Repositorio.

\section{Evaluación de impacto:}

En Redbanc cada iniciativa de negocio es evaluada previamente tanto desde el punto de vista Estratégico-Comercial como del punto de vista Técnico-Operativo, éste último con el objetivo de dimensionar los posibles impactos en las aplicaciones, procesos y seguridad que la implementación de dichas iniciativas implica. En este punto es de mucha utilidad contar con un Modelo Arquitectónico que permita visualizar las relaciones y la trazabilidad entre las diferentes entidades relacionadas en la implementación de una iniciativa. 


\section{$(1)$}

Dado que la complejidad y costo de hacer cambios en las implementaciones incrementa a medida de que se avanza en las etapas de la ejecución de un proyecto es que la identificación anticipada de los impactos se convierte en una fortaleza al momento de dimensionar el tamaño, costo y duración de los proyectos asociados. Con el uso de un modelo que relacione todas las entidades de negocio de la organización se disminuye considerablemente la posibilidad de omitir algún impacto en el análisis temprano de las iniciativas a desarrollar.

\section{Metodologías Ágiles}

Para una metodología de desarrollo de software existen varias definiciones, sin embargo existen algunas ya establecidas por algunas empresas, especialistas y compañías que se dedican a este trabajo. Un ejemplo de esto es Avison y Fitzgerald (1995), nos presentan una definición de las metodologías de desarrollo indicando "Una metodología es una colección de procedimientos, técnicas, herramientas y documentos auxiliares que ayudan a los desarrolladores de software en sus esfuerzos por implementar nuevos sistemas de información".

Por otra parte, en la revista electrónica International Journal of Computer Applications define una metodología de desarrollo como "un proceso mediante el cual un proyecto de software es completado o desarrollado a través de procesos o etapas bien definidas". (Chandra, 2015).

Tal como indican Navarro, Fernández y Morales (2013), en la década de los noventa surgieron metodologías de desarrollo de software ligeras, que más adelante fueron llamadas ágiles, las cuales tienen el fin de reducir la probabilidad del fracaso por subvaloración de costos, tiempos y funcionalidades en los proyectos de desarrollo de software. Se iniciaron como alternativa a las metodologías tradicionales, precisamente para reducir la carga burocrática propia de ellas. A diferencia de las metodologías tradicionales, las metodologías ágiles son adaptativas y están orientadas a las personas.

Las metodologías ágiles se basan en un enfoque flexible, pueden ser modificadas para que se ajusten a la realidad de cada equipo y proyecto. Los proyectos ágiles se subdividen en 


\section{(1)}

proyecto más pequeños mediante una lista ordenada de características. Cada proyecto se trabaja de manera independiente y desarrolla un subconjunto de características durante un periodo de tiempo corto, de entre dos o seis semanas. La comunicación con el cliente es constante al punto de requerir un representante de él durante el desarrollo. Los proyectos son colaborativos y se acoplan a los cambios. El cambio en los requerimientos es una característica esperada y deseada, al igual que las entregas constantes al cliente y la retroalimentación por parte de él.

Al realizar una comparación entre las metodologías se puede evidenciar que existen varios aspectos relevantes entre ambas.

\begin{tabular}{|c|c|}
\hline Metodologias tradicionales & Metodologias ágiles \\
\hline Predictivos & Adaptativos \\
\hline Orientados a procesos & Orientados a personas \\
\hline Proceso rígido & Proceso flexible \\
\hline Se concibe como un proyecto & $\begin{array}{l}\text { Un proyecto es subdividido } \\
\text { en varios proyectos más } \\
\text { pequeños }\end{array}$ \\
\hline $\begin{array}{l}\text { Poca comunicación con el } \\
\text { cliente }\end{array}$ & $\begin{array}{l}\text { Comunicación constante con } \\
\text { el cliente }\end{array}$ \\
\hline $\begin{array}{l}\text { Entrega de software al } \\
\text { finalizar el desarrollo }\end{array}$ & $\begin{array}{l}\text { Entregas constantes de } \\
\text { software }\end{array}$ \\
\hline Documentación extensa & Poca documentación \\
\hline
\end{tabular}

Figura 6. Metodologías tradicionales vs metodologías ágiles. Adaptado de "Revisión de metodologías ágiles para el desarrollo de software”, por Prospectiva, 2013.

\section{Planificación}

Con respecto a la planificación que existe en cada metodología, se puede indicar que la planificación ágil hace referencia a la planificación en función de objetivos de negocio en lugar de tareas (a diferencia de la planificación tradicional) priorizando los que aportan más valor. Según lo indicado en la web de Proyectosagiles.org (“s.f.”), la planificación ágil toma como base el control empírico de la construcción del producto (inspección y adaptación) por lo cual: 


\section{(1)}

$\checkmark$ Se plantea fases en base a objetivos de producto priorizado en base a los beneficios del negocio respecto a sus costos de desarrollo.

$\checkmark$ Fases de 2 a 4 semanas con demostraciones al cliente de ese incremento de producto.

$\checkmark$ Elabora retrospectivas durante todo el proyecto, mejorando la productividad y calidad dentro del propio proyecto.

$\checkmark$ Hace que todo el equipo participe durante el proceso.

A continuación se podrá observar más detalle sobre la planificación ágil vs la planificación tradicional

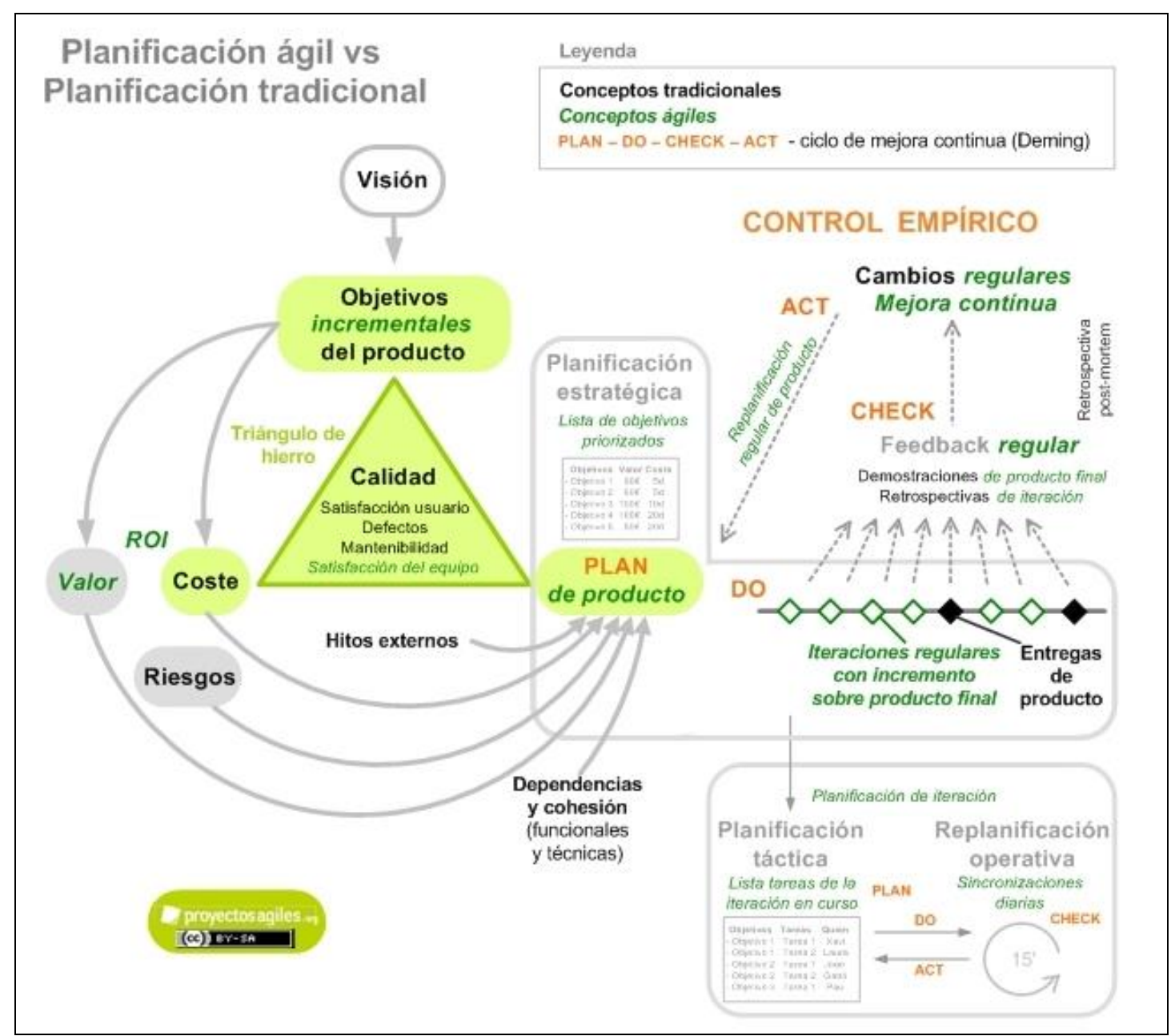

Figura 7. Planificación ágil vs tradicional. Adaptado de "Planificación ágil vs planificación tradicional", por Proyectosagiles.org, 2010. 


\section{(1)}

\section{Metodologías ágiles representativas}

Tal como indican Navarro, Fernández y Morales (2013), las metodologías ágiles más representativas se detallan a continuación:

Scrum: Para el desarrollo ágil de software es un marco de trabajo diseñado para lograr la colaboración eficaz de equipos en proyectos, que utiliza un conjunto de reglas y artefactos y define roles que generan la estructura necesaria para su funcionamiento correcto.

Extreme Programming (XP): Es una metodología ágil centrada en guiar equipos de desarrollo de software pequeños o medianos, entre dos y diez desarrolladores, en ambientes de requerimientos imprecisos o cambiantes. Tiene como base cinco valores: Simplicidad, Comunicación, Retroalimentación, Respeto y Coraje.

Además existen otras metodologías relevantes las cuales se detallan:

Crystal: Es una metodología basada en los conceptos de Rational Unified Process (RUP) y está compuesta por Crystal Clear, Crystal Yellow, Crystal Orange y Crystal Red; el nivel de opacidad del color hace referencia a un mayor número de personas implicadas en el desarrollo, un mayor tamaño de proyecto y por ende un mayor control en el proceso.

Método de desarrollo de sistemas dinámicos: Es un marco de trabajo empleado para entregar la solución correcta en el momento correcto. Utiliza un ciclo de vida iterativo, divide el proyecto en periodos cortos y define entregables para cada uno de estos.

Desarrollo adaptativo de software: Se basa en la teoría de sistemas adaptativos complejos. Debido a ello, interpreta los proyectos de software como sistemas adaptativos complejos compuestos por agentes (interesados), entornos (organizacional), tecnológicos y salidos (producto desarrollado). Sus fases son: especulación, colaboración y aprendizaje.

Desarrollo orientado a funcionalidades: Se basa en la planeación y el diseño adelantado. Debido a ello, el modelo de objetos, la lista de características y la planeación se hacen al inicio del proyecto. Las iteraciones son incrementos con características identificadas.

\section{Marco de Trabajo: SCRUM}

Tal como indican Navarro, Fernández \& Morales (2013), SCRUM utiliza un enfoque incremental que tiene como fundamento la teoría de control empírico de procesos. Dicha 


\section{(1)}

teoría se basa en la transparencia, inspección y adaptación; la transparencia , que avala la visibilidad en el proceso de las cosas que pueden afectar el resultado; la inspección, que ayuda a descubrir variaciones indeseables en el proceso; y la adaptación, que realiza los ajustes pertinentes para minimizar el impacto de las mismas.

En Scrum se manejan equipos los cuales son auto-gestionados, multifuncionales y trabajan en iteraciones. La entrega del producto se hace en iteraciones; cada iteración crea nueva funcionalidades o modifica las que el dueño del producto requiera.

Adicionalmente, Scrum también maneja ciertos roles y responsabilidades, donde el marco de trabajo requiere de tres pero pueden existir roles adicionales.

Los roles y responsabilidades de Scrum son:

- El Scrum master

- El Producto Owner o dueño del producto

- El equipo de desarrollo

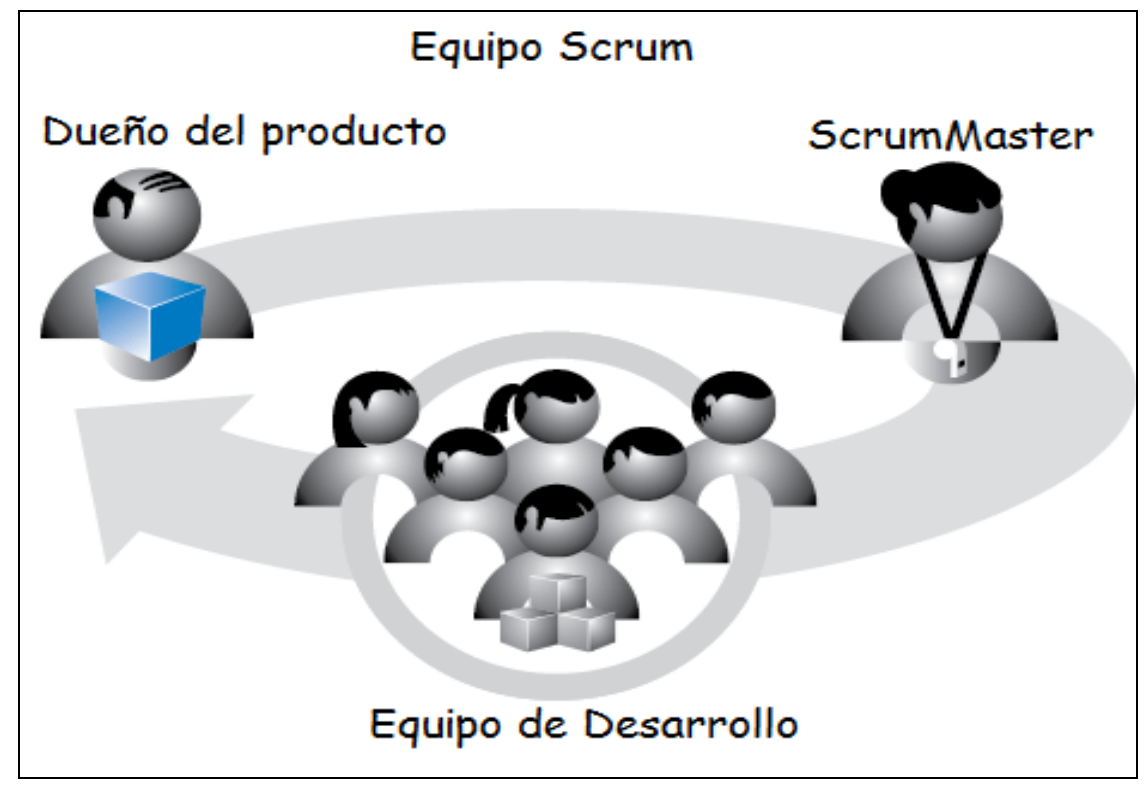

Figura 8. Equipo Scrum. Adaptado de "Scrum roles y responsabilidades", por Kenneth Rubin, 2018.

Scrum maneja 5 eventos para fomentar la colaboración y comunicación del equipo reduciendo así las reuniones extensas y los procesos restrictivos y predictivos. Aparte de ello se utilizan para minimizar la necesidad de reuniones no definidas. Los siguientes eventos 


\section{(1)}

pueden terminar siempre que se logre el propósito del evento, pero dentro de la caja de tiempo y asegurando el fomento de la transparencia.

Los eventos de Scrum son:

- El evento principal o Sprint

- Planificación del Sprint o Sprint Planning

- Daily Scrum

- La revisión del Sprint o Sprint Review

- La Retrospectiva del Sprint o Sprint Retrospective

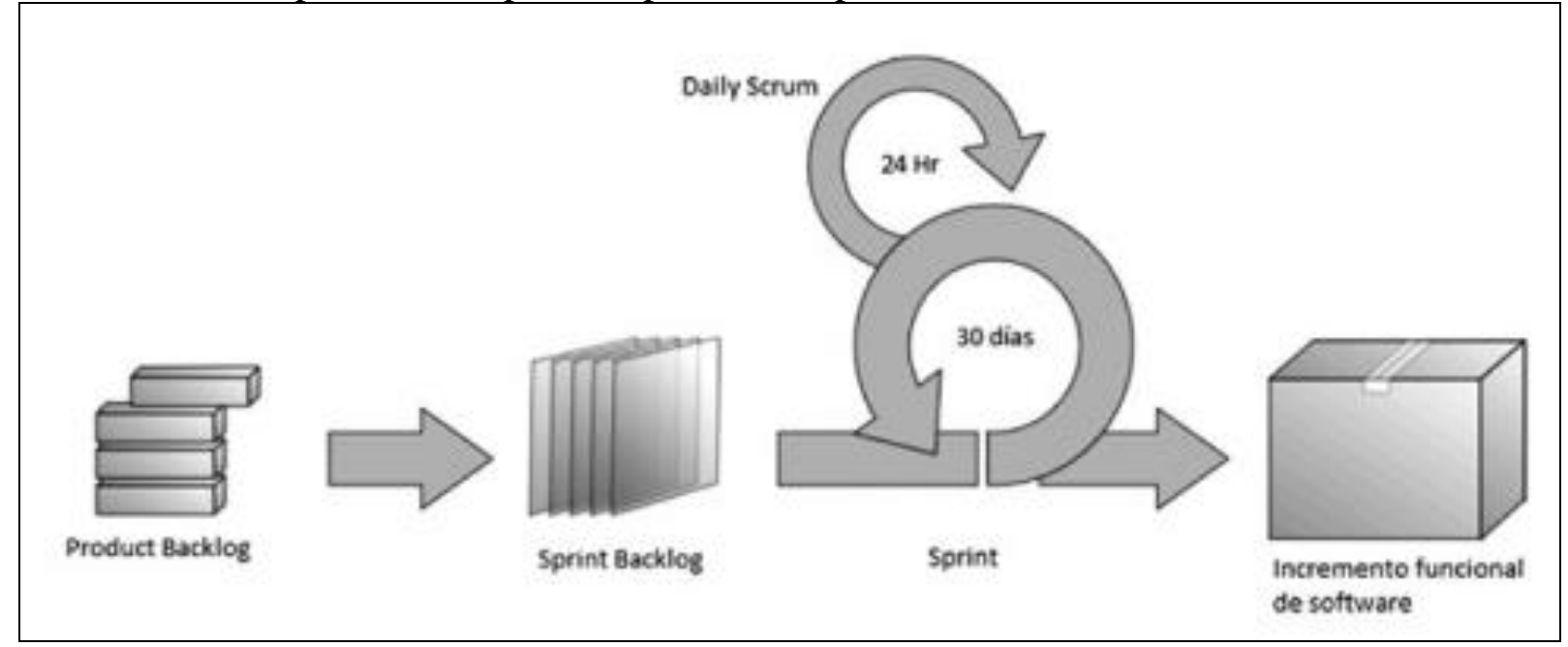

Figura 9. Fases de un Sprint. Adaptado de "Metodología Scrum: Fases de un Sprint", por Andrés Navarro, Juan Fernández \& Juan Morales, 2013.

En Scrum existen también los artefactos, estos son subproductos de las actividades del marco de trabajo que le brindan dirección y transparencia al equipo. Los artefactos Scrum son:

- El Product Backlog

- El Sprint Backlog

- El Monitoreo de Progreso

- El Incremento

El ciclo de vida de este marco de trabajo está compuesto de cuatro fases:

Planeación, en la cual se establece la visión, se fijan las expectativas y se asegura el financiamiento. 


\section{(1)}

Puesta en escena, en la cual se identifican más requerimientos y se priorizan para la primera iteración.

Desarrolla, en la cual se desarrolla el sistema.

Entrega, en la cual se hace el despliegue operativo.

Tal como indican Schwaber \& Sutherland (2016), cuando el Equipo Scrum incorpora y vivencia los valores de compromiso, coraje, foco, apertura y respeto, los pilares de Scrum de transparencia, inspección y adaptación se materializan y fomentan la confianza en todo. Los miembros del Equipo Scrum aprenden y examinan estos valores a medida que trabajan en los eventos, roles y artefactos de Scrum. El éxito de Scrum depende de que las personas lleguen a ser más virtuosas en la convivencia con estos cinco valores. Las personas se comprometen de manera individual a alcanzar las metas del Equipo Scrum. Los miembros del Equipo Scrum tienen coraje para hacer las cosas bien y para trabajar en los problemas difíciles. Todos se centran en el trabajo del Sprint y en las metas del Equipo Scrum. El Equipo Scrum y sus interesados acuerdan estar abiertos a todo el trabajo y a los desafíos que se les presenten al realizar su trabajo. Los miembros del Equipo Scrum se respetan entre sí para ser personas capaces e independientes.

Por último, el marco de trabajo Scrum consiste en los Equipos Scrum y sus roles, eventos, artefactos y reglas asociadas. Cada componente dentro del marco de trabajo sirve a un propósito específico y es esencial para el éxito de Scrum y para su uso. Las reglas de Scrum relacionan los eventos, roles y artefactos, gobernando las relaciones e interacciones entre ellos.

A continuación, se muestra como se relaciona lo mencionado anteriormente: 


\section{(1)}

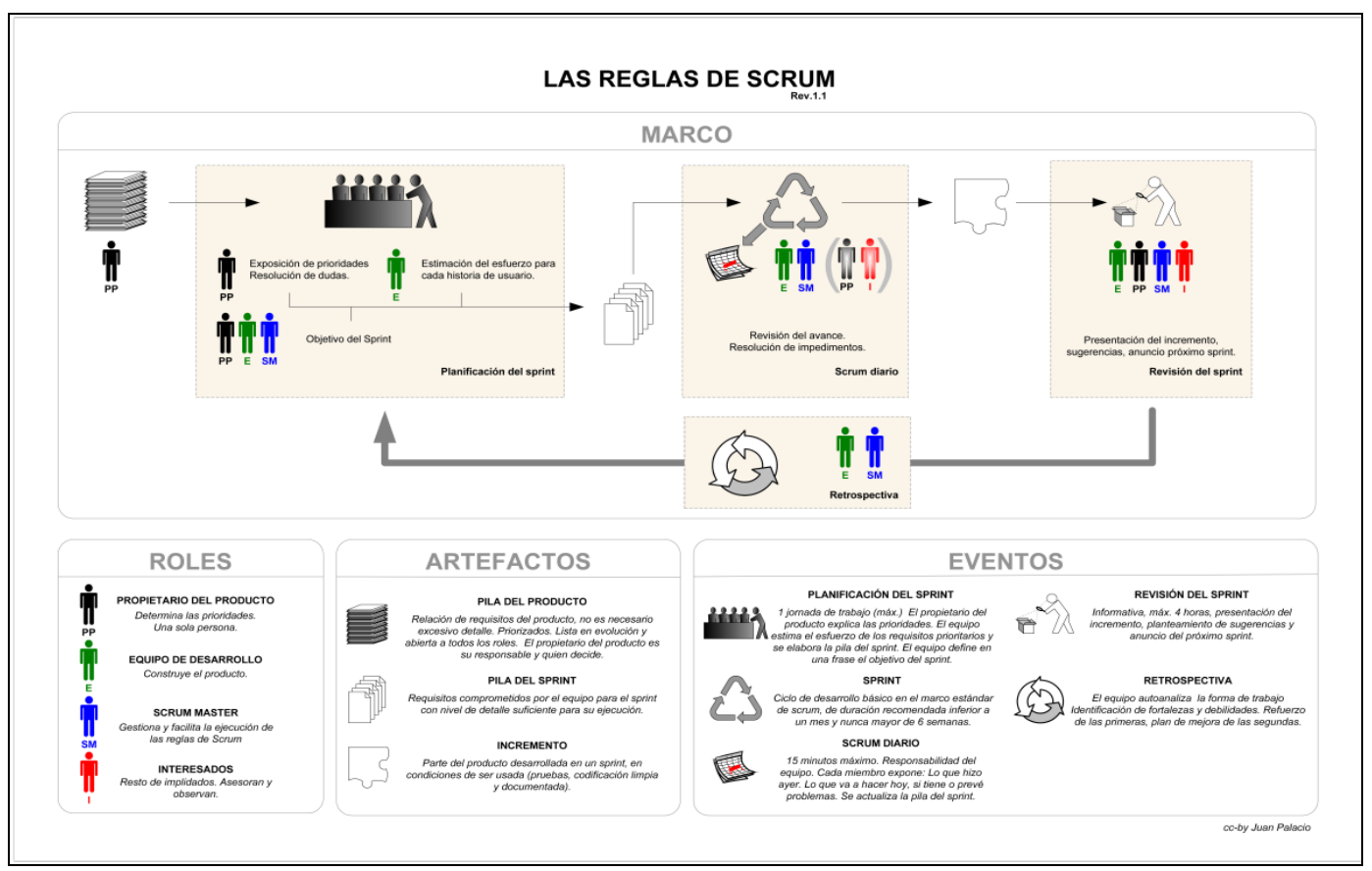

Figura 10. Las reglas del SCRUM. Adaptado de "Scrum Manager I: Las reglas de scrum", por Juan Palacio, 2015.

\section{Beneficios del marco de trabajo Scrum}

Según lo indicado en la web de Proyectosagiles.org ("s.f."), existen varios beneficios del marco de trabajo Scrum, pero dentro de los principales se encuentran los siguientes:

- Poder realizar entregas mensuales/quincenales de los resultados, esto conlleva a:

- Obtener resultados tangibles y poder gestionar las expectativas del cliente.

○ Obtener resultados anticipados.

- Ante cualquier cambio en el negocio o respecto a las necesidades del cliente existe gran flexibilidad y adaptación.

○ Retorno de Inversión gestionable.

○ Riesgos del proyecto mitigados.

- Incrementar la productividad y calidad

- Poder estar enfocados hacia un mismo objetivo tanto el equipo de desarrollo como el cliente.

- Contar con un equipo de trabajo motivado. 


\section{(}

\section{CASO DE ÉXITO}

Según lo indicado por Goikolea (2014), La empresa Spotify ha sabido adaptarse perfectamente a las metodologías ágiles, haciendo especial enfoque en la figura del Scrum Master. Muchas veces contratan un Agile Coach externo con una gran experiencia en el campo para liderar los proyectos. Vemos aquí la importancia de contar con roles especializados que conozcan las metodologías ágiles para llevar un proyecto de este tipo al éxito. Ya no solo el Scrum Master, sino también otros roles como el Product Owner, responsable de entender al cliente y al usuario para saber trasladar en tiempo y forma la información adecuada al equipo de desarrollo. Spotify es consciente de la metodología de trabajo de su competencia (Google o Apple por ejemplo), por lo que decidieron acercarse al Scrum de forma muy sistemática. Compitiendo contra similares corporaciones, sabían que en cualquier momento podrían ser derrotados a menos que fuesen más rápidos, más baratos y mejores.

En Spotify los equipos se organizan por escuadrones, que son pequeños equipos de Scrum con la habilidad de implementar el software desarrollado al final de cada sprint, sin romper ningún otro equipo. Una característica curiosa del funcionamiento de Spotify es que cada uno de estos pequeños grupos tiene una parte del producto que es totalmente suyo. Después crean tribus agregando distintos escuadrones. Aun así, Spotify necesita implementar, cambiar y actualizar su código constantemente sin romper nada más. Para ello es necesaria una buena coordinación central de la compañía. Si se quiere ser rápido también es necesario deshacerse de todas aquellas partes del proceso que entorpecen el avance. En Spotify, por ejemplo, se contaba con un equipo de operaciones que se encargaba de las implementaciones, pero el funcionamiento era demasiado lento. Por eso decidieron eliminar esta fase y hacer que los propios desarrolladores implementasen sus trabajos.

\section{JIRA Software}

Según lo indicado por Atlassian (2002), El Jira es una herramienta para la planificación de proyectos o de gestión de entornos colaborativos que optimiza la productividad de los equipos de trabajo. Puede utilizarse como un software de gestión de tareas cuando se necesita una estructura, agrupación y flujo de trabajo determinado. Fue desarrollado en lenguaje 


\section{(1)}

JAVA EE y que puede funcionar con diversas bases de datos y sistemas operativos, inicialmente el JIRA se solía usar como una herramienta de seguimiento de incidencias sin embargo, con la madurez de la empresa se empezó a usar para la gestión y mejora de los procesos. De forma nativa, puede llevarse un registro de horas sobre las tareas y su gestión suele hacerse a través de paneles kanban o gráficos de gantt. Soporta plugins que permiten expandir sus capacidades.

El JIRA nos permite realizar las siguientes actividades:

- Planificar: Crea historias de usuario e incidencias, planifica sprints y distribuye tareas entre tu equipo de software.

- Supervisar: Prioriza y analiza el trabajo de tu equipo en su contexto y con una completa visibilidad.

- Lanzar: Realiza lanzamientos con confianza y seguridad, sabiendo que la información que tienes es siempre la más actualizada.

- Crear informes: Mejora el rendimiento del equipo con datos visuales en tiempo real que tu equipo puede emplear.

Aparte de ello, en caso seas una empresa experta, avanzado o un principiante el Jira ofrece también todo sobre metodologías ágiles como:

- Planificación flexible: El equipo podrá planificar con flexibilidad de la manera que les resulte más conveniente, cuenta con Scrum, Kanban o metodología mixta.

- Estimaciones precisas: Ayudan al equipo a trabajar con más precisión y eficacia. Se puede utilizar puntos de historia, horas, tallas o tu propia técnica de estimación.

- Priorización basada en valores: Ordena incidencias, historias de usuario y errores en el backlog del producto con solo moverlos dependiendo de la prioridad.

- Ejecución transparente: Independientemente donde se encuentre el equipo Jira ofrece un nivel superior de transparencia sobre el trabajo de cada equipo y mantiene a la organización en sintonía.

- Resultados prácticos: Cuenta con creación de informes, el cual contiene información sobre el equipo utilizando los procedimientos ágiles.

- Evolución escalable: Se puede cambiar y añadir campos, y tipos de flujos de trabajo a medida que lo requieran, ofrece también una gestión de proyectos ágiles para equipos de cualquier tamaño y condición. 
CAPÍTULO 3: ANÁLISIS DEL PROYECTO 


\section{(4)}

\section{ARQUITECTURA EMPRESARIAL}

\section{PRELIMINAR}

Se tendrá que desarrollar el siguiente entregable:

- Petición de Trabajo de Arquitectura (Request for Architecture work), el cual contiene:

\section{LIMITES DE TIEMPO}

- Todo proyecto interno a ejecutarse tiene como tiempo promedio el plazo de un año, este tiempo puede variar dependiendo de la complejidad del caso.

\section{LIMITACIONES ORGANIZACIONALES / LIMITACIONES DE NEGOCIO}

- Se cuenta actualmente con el tercer nivel de certificación del modelo CMMI y con certificación internacional de calidad ISO 9001:2008.

\section{LIMITACIONES FINANCIERAS}

- El Gerente de Administración y Finanzas maneja un presupuesto para desarrollo de proyectos, el monto es de 500 mil nuevos soles (S/. 500,000.00).

\section{LIMITACIONES EXTERNAS}

- Cumplimiento de las normativas establecidas por los entes reguladores (Superintendencia de Banca y Seguros, y marcas de tarjetas internacionales) respecto al soporte de la tarjeta Chip (EMV/PCI).

\section{DESCRIPCIÓN DE LA SITUACIÓN ACTUAL DEL NEGOCIO}

El proceso inicia cuando el Consultor de Negocio (Marketing) genera el código del proyecto en el Sistema SARA, este código se generará tanto para mejoras o nuevos proyectos. Una vez generado el código el Sistema SARA emitirá un correo dirigido al Gerente de Operaciones 


\section{(1)}

con las especificaciones básicas del proyecto (Código del proyecto, Cliente, contacto cliente, duración esperada).

El Gerente de Operaciones define el o las área(s) (C - STP, Java - CAT) que participaran en el proyecto. Luego se reunirán el o los Gerente(s) de área(s) para definir los recursos que serán asignados al proyecto. En caso no se cuente con los recursos disponibles, se evaluará la contratación de programadores externos. Una vez seleccionado el equipo de proyecto, se realizara las reuniones con el cliente para poder establecer y definir los requerimientos funcionales y no funcionales teniendo en consideración las reglas del negocio. Dichas reuniones se llevaran a cabo con el Gerente de área, Líder técnico y el cliente, en las cuales el Líder técnico deberá enviar un correo con los temas acordados a los participantes de la reunión.

El Líder técnico iniciará el ciclo del proyecto elaborando el cronograma de trabajo de acuerdo al tipo de proyecto (Atención de Requerimiento / intermedio y avanzado), al finalizar cada etapa la Administradora de la configuración deberá guardar los documentos ingresados por el líder técnico, los cuales previamente han sido verificados. El Líder técnico solicitará por medio de un correo electrónico la verificación de documentos del proyecto al Jefe de Control, el cual designara a la Analista de calidad la verificación de los documentos de acuerdo a los formatos ya establecidos en la empresa. En caso no pase la verificación el Analista de Calidad enviara un correo al Líder técnico con las observaciones respectivas. El Líder técnico recibirá el correo y levantara las observaciones, luego este enviara un correo al Analista de calidad con las observaciones corregidas y el documento actualizado. En caso se encuentren más observaciones deberá seguir el mismo flujo, caso contrario el Analista de Calidad enviara el correo de conformidad de verificación del documento. Dicho correo se debe adjuntar cuando el Líder técnico envíe un correo al Administrador de la configuración, el cual siempre debe verificar el correo de conformidad del Analista de Calidad antes de guardar el documento en el servidor de la empresa.

En el cronograma se asignaran las tareas y el esfuerzo para cada recurso, el Líder técnico deberá ingresar las actividades del cronograma y asignar a cada recurso en forma manual, luego de ello utilizará el sistema IMPORT/EXPORT, el cual cargará las actividades al sistema SARA (import) para que los recursos asignados puedan visualizar e ingresar su avance de tareas semanales. 


\section{(1)}

El Líder técnico, todos los jueves deberá actualizar el cronograma según el avance registrado por el recurso en el sistema SARA, para ello deberá bajar la información (export) y comparar con el cronograma del anterior jueves para que pueda visualizar si hay desviaciones, si se debe agregar o disminuir duración o esfuerzo de alguna actividad dependiendo de los cambios o nuevos requerimientos que puedan existir. La asistente de proyectos recibirá el cronograma actualizado por el Líder técnico y utilizara el Sistema Procesador de Cronogramas, el cual validará si existe algún cambio o variación con lo planificado, de ser así, se generará una Solicitud de cambio ya sea por los motivos de: esfuerzo, duración o hitos, dicha solicitud pasara un proceso de aprobación por el gerente de área, líder técnico, gerente de Cuenta, cada aprobación será validada con la firma de cada Gerente. Además, deberá elaborar o actualizar todos los entregables de gestión que genera el proceso.

Por último se cumplirá con cada etapa del ciclo del proyecto y se realizara un Informe de Cierre, El Gerente de Operaciones lo revisará y evaluara los tiempos y desviaciones del proyecto durante todo el ciclo de vida del proyecto. Dicho Informe será aprobado por la gerencia y se dará por cerrado el proyecto.

El proceso de Gestión de Proyectos tiene por objetivo:

1. Gestionar los requerimientos a lo largo del ciclo de vida del proyecto, mantener la trazabilidad de los requerimientos y administrar cambios en los requerimientos.

2. Monitorear las actividades, compromisos, riesgos, involucrados directos e indirectos del proyecto. Asimismo tomar las acciones correctivas de acuerdo al seguimiento que se realiza del proyecto.

3. Identificar riesgos a lo largo del proyecto, definir estrategias, evaluarlos, priorizarlos y desarrollar los planes de mitigación y/o contingencia para afrontar el riesgo.

4. Integrar planes (actividades y recursos) para coordinar cruces y dependencias y realizar una coordinación integral.

5. Establecer las guías y criterios para tomar una decisión de acuerdo a las alternativas y métodos de evaluación definidos. 


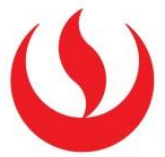

Tabla 3

Proceso vs Problemas

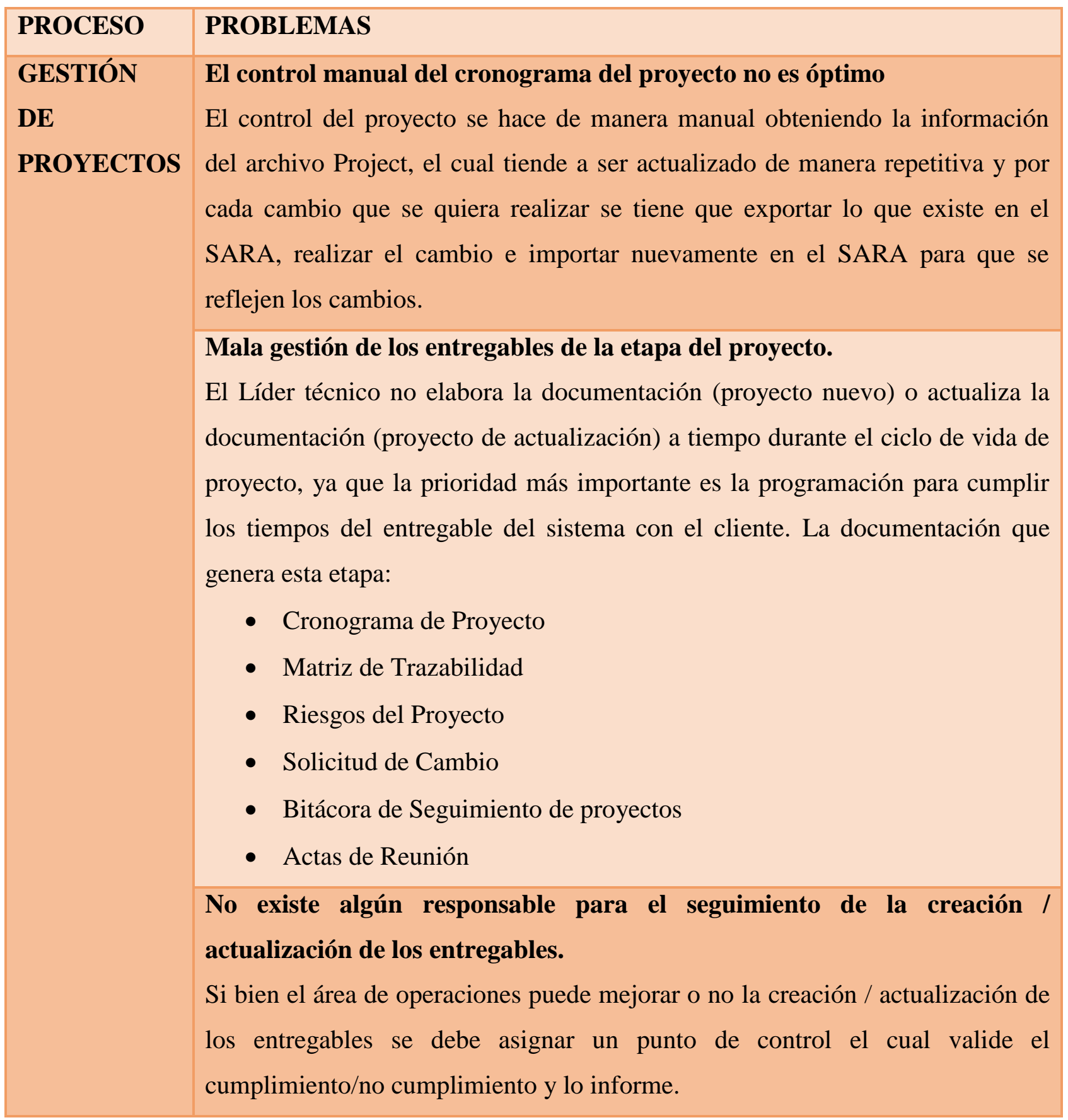

Nota: Se ha identificado los problemas existentes en el proceso de gestión de proyectos. Adaptado de

"Procedimiento de Gestión de Proyecto", por El Objeto de Estudio, 2017. 


\section{(1)}

\section{DESCRIPCIÓN DE LA SITUACIÓN ACTUAL DE LA ARQUITECTURA/TI}

Las aplicaciones que actualmente se utilizan para dar soporte al proceso de Gestión de Proyectos son las siguientes:

- $\underline{\text { Sistema SARA }}$

Contiene información sobre las áreas, los productos, los proyectos, y trabajadores de la empresa, las actividades de proyectos solo las ingresan el personal del área de Operaciones y Control.

\section{- $\underline{\text { Sistema IMPORT/EXPORT }}$}

Se encarga de sincronizar las actividades del cronograma con el Sistema SARA (Importación), el sistema deberá leer un archivo Project y exportarlo con información actualizada del sistema SARA (Exportación).

- $\underline{\text { Sistema Procesador de Cronogramas }}$

Se encarga de procesar los cronogramas e identificar si existen variaciones con lo planificado, de ser así, se generará una Solicitud de cambio ya sea por los motivos de: esfuerzo, duración o hitos.

Tabla 4

Sistemas vs Problemas

\begin{tabular}{|l|l|}
\hline SISTEMAS & PROBLEMAS \\
\hline Sistema SARA & Lenguaje de programación obsoleto \\
& El sistema SARA es más utilizado como un repositorio de datos con \\
el cual se comunican los otros subsistemas existentes de la empresa. & \\
& No se realizan mejoras o nuevos módulos ya que la Gerencia no \\
desea invertir en tecnología antigua.
\end{tabular}




\section{(1)}

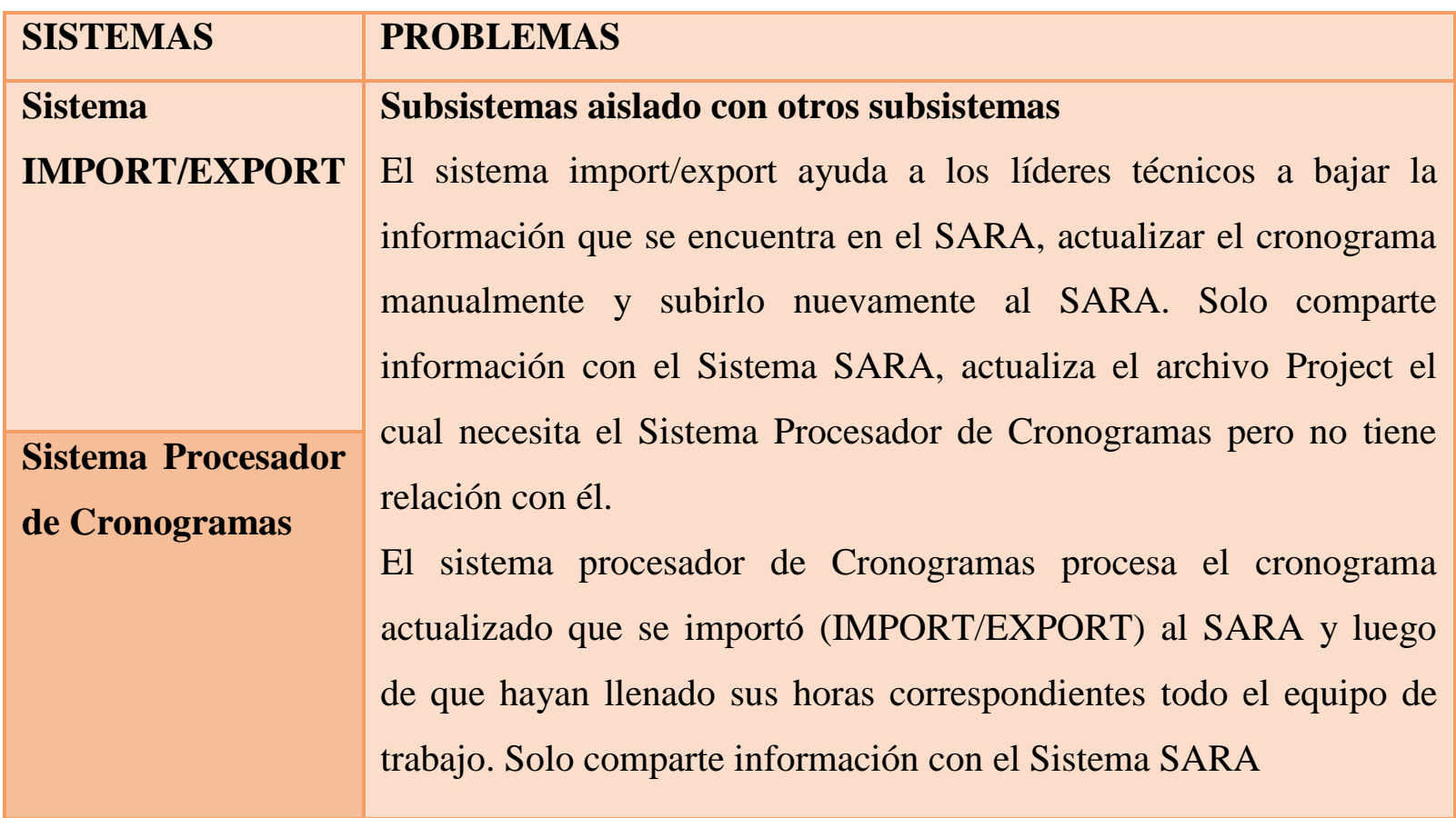

Nota: Se ha identificado los problemas existentes en los sistemas del proceso de gestión de proyectos. Adaptado de "Procedimiento de Gestión de Proyecto", por El Objeto de Estudio, 2017.

\section{ARQUITECTURAS (AS IS / TO BE)}

Se tendrá que desarrollar el siguiente entregable:

- Documento de Definición de Arquitectura (Architecture Definition Document), el cual contiene:

\section{ALCANCE}

La consultora de software es una compañía de tecnología de información que brinda servicios de desarrollo de aplicaciones, integración de sistemas, gerencia de proyectos y consultoría. Esta propuesta arquitectura se focalizará en el proceso de Gestión de Proyectos que está relacionado con el ciclo de vida de un proyecto interno o externo. El periodo de duración considerado es de tres (3) meses. La propuesta de arquitectura abarca los cuatro dominios de la AE: Negocio, Aplicaciones, Datos y Tecnológica. 


\section{(1)}

De acuerdo al Método de Desarrollo de la Arquitectura (ADM), establecida por TOGAF, el proyecto comprende desde la fase "Preliminar" hasta la fase "Oportunidades y Soluciones", considerando los siguientes entregables:

- Petición de Trabajo de Arquitectura

- Documento de Definición de Arquitectura

- Plan de Implementación de la Migración

Los trabajos de implementación están fuera del alcance del presente proyecto.

\section{PRINCIPIOS DE ARQUITECTURA}

\section{PRINCIPIOS DE NEGOCIO}

Tabla 5

Principio de Negocio - Satisfacción del cliente

\begin{tabular}{|l|l|}
\hline Nombre & Satisfacción del cliente \\
\hline Enunciado & $\begin{array}{l}\text { Lograr la satisfacción del cliente frente un producto o servicio brindado por } \\
\text { la empresa. }\end{array}$ \\
\hline Fundamento & $\begin{array}{l}\text { Saber si lo requerido por el cliente se cumplió de manera correcta logrando } \\
\text { su aceptación de la solución. }\end{array}$ \\
\hline Repercusiones & $\begin{array}{l}\text { El no lograr la satisfacción del cliente genera que la empresa no se muestre } \\
\text { como un proveedor potencial generando su reducción de participación en el } \\
\text { mercado. }\end{array}$ \\
\hline
\end{tabular}

Nota: Se ha descrito el detalle, fundamento y repercusión del principio de negocio - satisfacción del cliente.

Adaptado de "Manual de calidad", por El Objeto de Estudio, 2017.

Tabla 6

Principio de Negocio - Creación y actualización de productos

\begin{tabular}{|l|l|}
\hline Nombre & Creación y actualización de productos \\
\hline Enunciado & $\begin{array}{l}\text { La empresa debe crear o actualizar por lo menos } 2 \text { proyectos de manera } \\
\text { anual. }\end{array}$ \\
\hline Fundamento & Generar nuevos productos o actualización de los ya existentes permite tener \\
\hline
\end{tabular}




\section{(4)}

\begin{tabular}{|l|l|}
\hline Nombre & Creación y actualización de productos \\
\hline mayor participación frente a clientes nuevos o antiguos. & \\
\hline Repercusiones & El no generar nuevos productos o actualización de estos genera \\
& estancamiento de la empresa en el mercado.
\end{tabular}

Nota: Se ha descrito el detalle, fundamento y repercusión del principio de negocio - creación y actualización de productos. Adaptado de "Manual de calidad", por El Objeto de Estudio, 2017.

Tabla 7

Principio de Negocio - Control de proyectos

\begin{tabular}{|l|l|}
\hline Nombre & Control de proyectos \\
\hline Enunciado & $\begin{array}{l}\text { Todo proyecto en curso debe ser controlado de alguna manera generando } \\
\text { informes de estado sobre tiempos, riesgos, cambios y otros. }\end{array}$ \\
\hline Fundamento & $\begin{array}{l}\text { Ayuda a la toma de decisiones oportunas frente a posibles riesgos o retrasos } \\
\text { que presente el proyecto en su curso. }\end{array}$ \\
\hline Repercusiones & $\begin{array}{l}\text { El no tener un control adecuado del estado del proyecto puede generar } \\
\text { retrasos en la elaboración de este, perjudicando a la empresa en la } \\
\text { presentación al cliente. }\end{array}$ \\
\hline
\end{tabular}

Nota: Se ha descrito el detalle, fundamento y repercusión del principio de negocio - control de proyectos.

Adaptado de "Manual de calidad", por El Objeto de Estudio, 2017.

\section{PRINCIPIOS DE DATOS}

Tabla 8

Principio de Datos - Información de procesos compartido

\begin{tabular}{|c|c|}
\hline Nombre & Información de procesos compartido \\
\hline Enunciado & $\begin{array}{l}\text { La información sobre los procesos que cuenta la empresa está a la } \\
\text { disposición de sus colaboradores. }\end{array}$ \\
\hline Fundamento & $\begin{array}{l}\text { Tener la información disponible a los colaboradores permite saber el flujo } \\
\text { adecuado de los procesos para una buena elaboración de estos. }\end{array}$ \\
\hline Repercusiones & $\begin{array}{l}\text { Si algún colaborador no conoce la forma correcta el cual se realiza los } \\
\text { procesos genera que este no se encuentre identificado con la empresa. }\end{array}$ \\
\hline
\end{tabular}

Nota: Se ha descrito el detalle, fundamento y repercusión del principio de datos - información de procesos compartido. Adaptado de "Manual de calidad", por El Objeto de Estudio, 2017. 


\section{(1)}

Tabla 9

Principio de Datos - Bitácora de seguimiento de proyectos

\begin{tabular}{|l|l|}
\hline Nombre & Bitácora de seguimiento de proyectos \\
\hline Enunciado & $\begin{array}{l}\text { Todo proyecto deberá manejar una bitácora de seguimiento para conocer } \\
\text { todos los eventos surgidos. }\end{array}$ \\
\hline Fundamento & $\begin{array}{l}\text { Conocer todos los posibles acuerdos, no conformidades, problemas, } \\
\text { lecciones aprendidas y mejoras de los diversos proyectos ejecutados y en } \\
\text { transcurso. }\end{array}$ \\
\hline Repercusiones & $\begin{array}{l}\text { No tener esta información limita que los posibles problemas presentados en } \\
\text { proyectos pasados pueden volver a surgir en los nuevos reduciendo la } \\
\text { eficiencia de la empresa. }\end{array}$ \\
\hline
\end{tabular}

Nota: Se ha descrito el detalle, fundamento y repercusión del principio de datos - bitácora de seguimiento de proyectos. Adaptado de "Manual de calidad", por El Objeto de Estudio, 2017.

Tabla 10

Principio de Datos - Copia de seguridad de información

\begin{tabular}{|l|l|}
\hline Nombre & Copia de seguridad de información \\
\hline Enunciado & $\begin{array}{l}\text { La información sobre los procesos, proyectos y fuentes de programas que } \\
\text { cuenta la empresa es reguardada ejecutando la copia de seguridad semanal. }\end{array}$ \\
\hline Fundamento & $\begin{array}{l}\text { Tener la información de la empresa resguardada y en caso ocurra algún } \\
\text { problema se pueda acceder a ella sin ningún problema. }\end{array}$ \\
\hline Repercusiones & $\begin{array}{l}\text { Si no se realiza la copia de seguridad y ocurre algún problema no se podrá } \\
\text { acceder a ninguna información de la empresa. }\end{array}$ \\
\hline
\end{tabular}

Nota: Se ha descrito el detalle, fundamento y repercusión del principio de datos - copia de seguridad de información. Adaptado de "Manual de calidad", por El Objeto de Estudio, 2017.

\section{PRINCIPIOS DE APLICACIONES}

Tabla 11

Principio de Aplicaciones - Facilidad de uso

\begin{tabular}{|l|l|}
\hline Nombre & Facilidad de uso \\
\hline Enunciado & Las aplicaciones que se usan son fáciles y prácticas. \\
\hline
\end{tabular}




\section{(1)}

\begin{tabular}{|l|l|}
\hline Nombre & Facilidad de uso \\
\hline Fundamento & $\begin{array}{l}\text { Mientras más fácil sean las aplicaciones que se usan, mejor será la } \\
\text { generación de la información para usar. }\end{array}$ \\
\hline Repercusiones & $\begin{array}{l}\text { Tener aplicaciones complejas puede generar errores involuntarios y generar } \\
\text { información errada. }\end{array}$ \\
\hline
\end{tabular}

Nota: Se ha descrito el detalle, fundamento y repercusión del principio de aplicaciones - facilidad de uso.

Adaptado de "Manual de calidad", por El Objeto de Estudio, 2017.

\section{PRINCIPIOS DE TECNOLOGÍA}

Tabla 12

Principio de Tecnología - Estándares y mejores prácticas

\begin{tabular}{|l|l|}
\hline Nombre & Estándares y mejores prácticas \\
\hline Enunciado & $\begin{array}{l}\text { Todo proyecto se realiza bajo los estándares establecidos por la empresa y } \\
\text { las mejores prácticas. }\end{array}$ \\
\hline Fundamento & $\begin{array}{l}\text { La empresa cuenta con certificados que le establecen estándares para la } \\
\text { elaboración de proyectos y realiza las actividades con las mejores prácticas } \\
\text { que se cuenta en la industria de TI. }\end{array}$ \\
\hline Repercusiones & $\begin{array}{l}\text { No aplicar los debidos estándares y mejores prácticas que cuenta la empresa } \\
\text { a los proyectos genera una baja calidad del producto o servicio al inicio, } \\
\text { durante o fin del proyecto. }\end{array}$ \\
\hline
\end{tabular}

Nota: Se ha descrito el detalle, fundamento y repercusión del principio de tenoclogía - estandares y mejores prácticas. Adaptado de "Manual de calidad", por El Objeto de Estudio, 2017. 
ARQUITECTURA DE LA LÍNEA DE BASE

1. ARQUITECTURA DE NEGOCIO

- ESTRUCTURA DE LA ORGANIZACIÓN

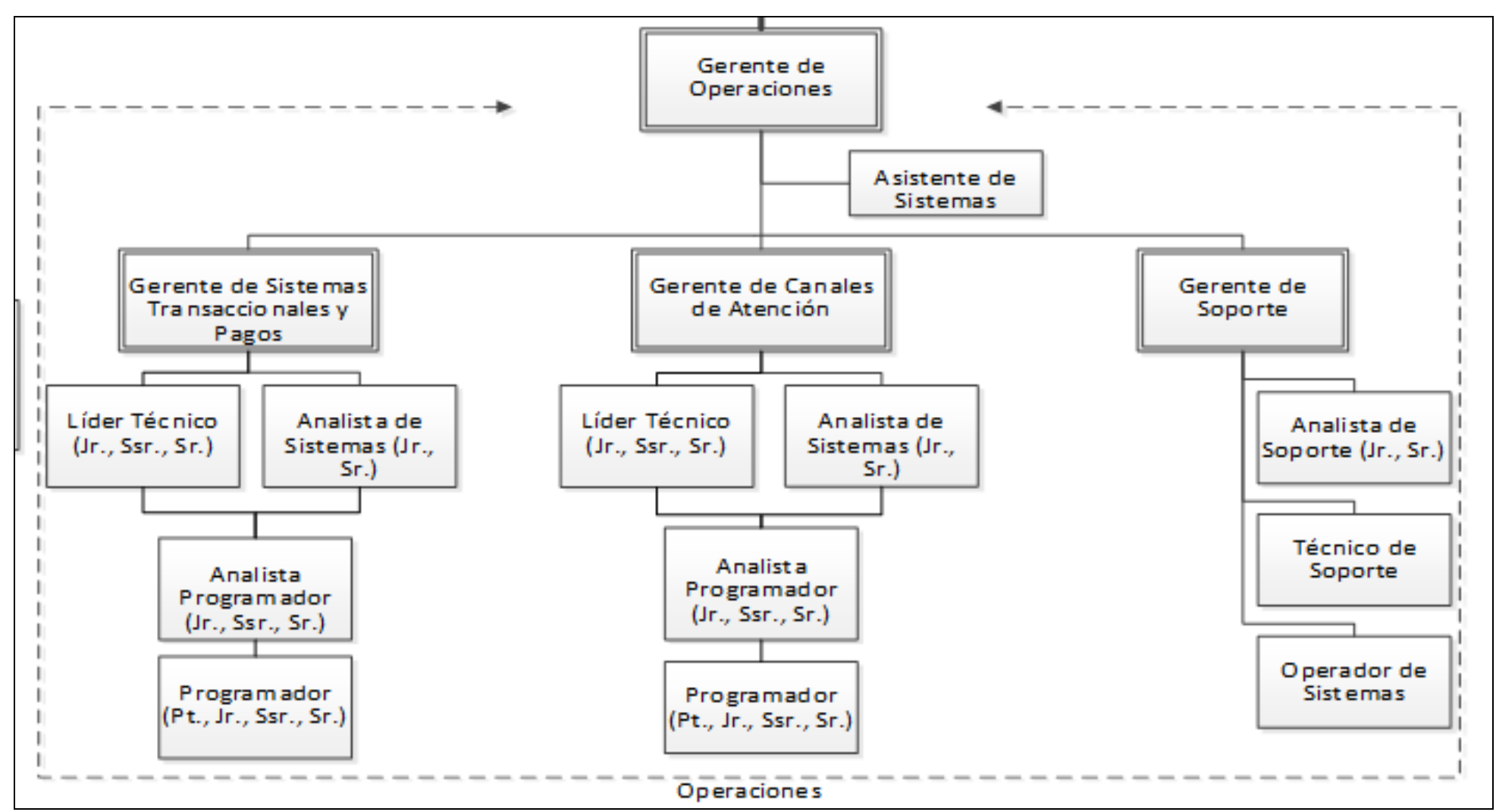

Figura 11. Organigrama de la empresa - Proceso Seleccionado (ASIS). Adaptado de "Manual de funciones y perfiles de puestos", por El Objeto de Estudio, 2017. 


\section{(1)}

Tal como indica el Objeto de Estudio (2017), a continuación se describen brevemente los roles indicados en el organigrama:

\section{Gerencia de Operaciones}

1.1. Gerente de Operaciones: Encargado de planificar, organizar, dirigir, y controlar las actividades y procedimientos de su Gerencia, con la finalidad de asegurar la administración eficiente de los recursos humanos, financieros, materiales y servicios requeridos para el correcto desarrollo de los proyectos y productos, así como brindar un óptimo mantenimiento y asistencia técnica a los clientes; contribuyendo así al cumplimiento de la misión y objetivos institucionales.

1.2. Asistente de Sistemas: Encargado de colaborar con las tareas administrativas y de gestión de las Gerencias de Producto, a fin de contribuir con el cumplimiento de los cronogramas y objetivos trazados para dichas áreas.

1.3. Gerente de Canales de Atención: Encargado de planificar, organizar, dirigir y controlar las actividades del área de Canales de Atención, con el objetivo de lograr la satisfacción de los clientes mediante una adecuada gestión de los proyectos y productos asignados así como del cumplimiento del Sistema de Gestión de la Calidad (SGC).

1.4. Gerente de Sistemas Transaccionales y Pagos: Encargado de planificar, organizar, dirigir y controlar las actividades del área de Sistemas de Pago, con el objetivo de lograr la satisfacción de los clientes mediante una adecuada gestión de los proyectos y productos asignados así como del cumplimiento del Sistema de Gestión de la Calidad (SGC).

1.5. Líder Técnico: Encargado de velar por el cumplimiento técnico de los productos, sistemas y servicios de la empresa, considerando los intereses / requerimientos técnicos tales como arquitectura, buenas prácticas y estándares de la empresa y de sus clientes. 
1.6. Analista de Sistemas: Encargado de analizar, diseñar, desarrollar e implementar productos y/o adecuaciones de los mismos cumpliendo las normas y procedimientos establecidos, a fin de satisfacer los requerimientos de los clientes e internos, garantizando la calidad del producto y la satisfacción del cliente.

1.7. Analista Programador: Encargado de analizar y describir los requerimientos para elaborar especificaciones funcionales y técnicas, así como ejecutar las actividades para convertir dichas especificaciones en un código de software, a fin de garantizar la calidad del producto y la satisfacción del cliente.

1.8. Programador: Encargado de ejecutar las tareas de programación e implementación de los proyectos y atenciones de requerimiento asignados aplicando los estándares de programación, las normas y procedimientos de la Institución para entregar un producto de calidad que cumpla con los requerimientos del cliente.

1.9. Gerente de Soporte: Encargado de brindar solución a los problemas que reporten los clientes y/o usuarios del Objeto de Estudio, de la manera más eficiente, en los tiempos acordados, proveyendo un soporte especializado y ágil con conocimiento y herramientas de TI, con el objetivo de asegurar un nivel de satisfacción elevado en los mismos.

1.10. Analista de Soporte: Encargado de realizar el análisis y corrección de los reportes de problemas reportados por los clientes, así como atender sus consultas en las implementaciones de producción, a fin de contribuir a que funcionen correctamente según lo definido en la documentación correspondiente.

1.11. Técnico de Soporte: Encargado de velar porque la infraestructura tecnológica de la empresa se encuentre operativa con el fin de permitir el desarrollo de las operaciones de la empresa.

1.12. Operador de Sistemas: Encargado de mantener la operatividad y continuidad del servicio "Sistema de procesamiento transaccional", a fin de garantizar en todo momento la disponibilidad del servicio frente a los clientes y autorizadores, en base al manejo de procedimientos y herramientas de gestión propias del sistema. 
○ MAPA DE PROCESOS Y FUNCIONES DE NEGOCIO

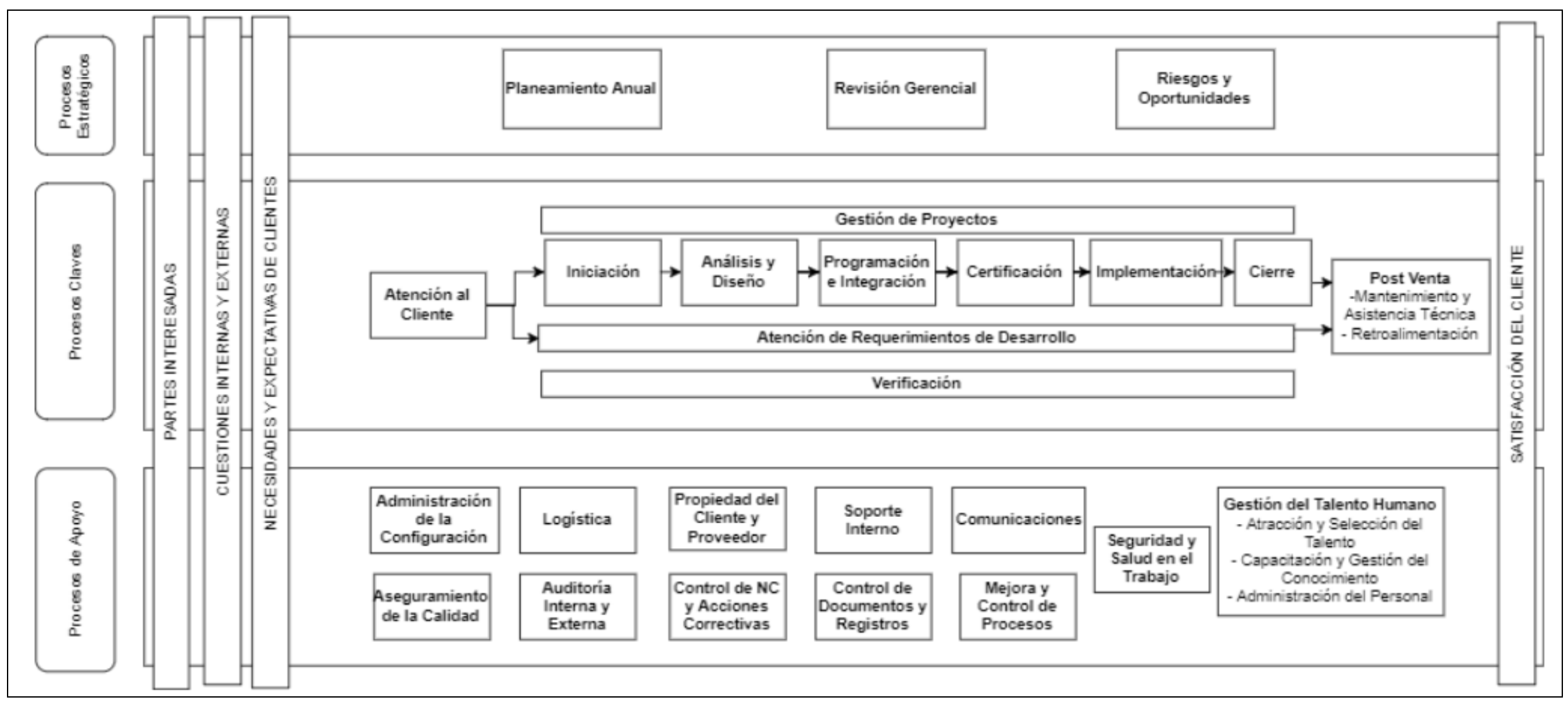

Figura 12. Mapa de procesos de la empresa (ASIS). Adaptado de "Manual de calidad", por El Objeto de Estudio, 2017. 


\section{(1)}

Tabla 13

Objetivos de los Procesos de Negocios (ASIS)

\begin{tabular}{|c|c|c|}
\hline $\mathbf{N}^{\mathbf{0}}$ & Procesos Claves & OBJETIVO DEL PROCESO \\
\hline 1 & Atención al Cliente & $\begin{array}{l}\text { Mantener una retroalimentación continua de las necesidades de los clientes: En } \\
\text { este proceso se elaboran las encuestas de satisfacción global y de proyectos, la } \\
\text { canalización de quejas y la atención de propuestas comerciales. } \\
\text { Recolectar requerimientos y necesidades de los clientes y elaborar propuestas } \\
\text { comerciales que le den frente. }\end{array}$ \\
\hline 2 & $\begin{array}{l}\text { Gestión } \\
\text { Proyectos }\end{array}$ & 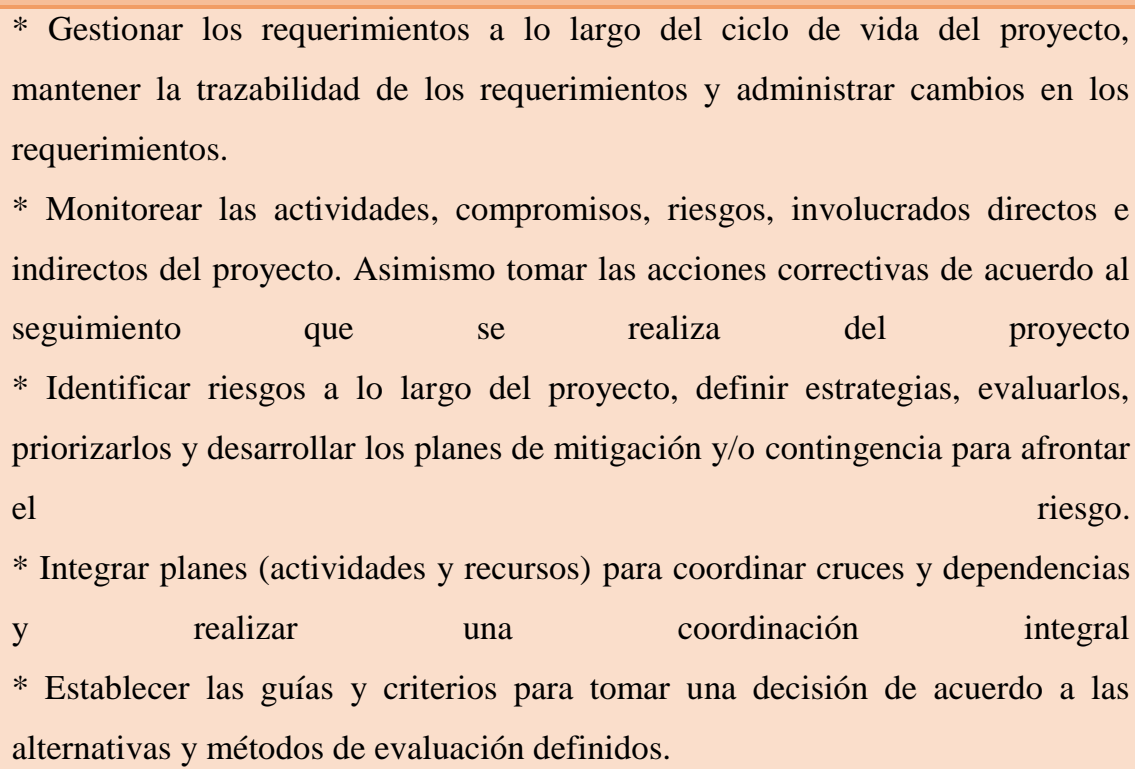 \\
\hline 3 & Iniciación & $\begin{array}{l}\text { * Definir el alcance, objetivos, actividades, recursos, conocimientos, } \\
\text { involucrados directos e indirectos que participan en el proyecto. } \\
\text { * Establecer } \\
\text { * Analizar con un mayor detalle los requerimientos del cliente para diseñarlos } \\
\text { en el proceso de Análisis y Diseño }\end{array}$ \\
\hline 4 & Análisis y Diseño & $\begin{array}{l}\text { * Elaborar el diseño en función de los requerimientos detallados en Iniciación } \\
\text { para establecer el esquema operacional, la funcionalidad requerida, las } \\
\text { interfaces } \\
\text { * necesarias, } \\
\text { funcionalidad requerida, en base a las cuales se tome una decisión, diseñar el } \\
\text { producto y sus componentes, diseñar las interfaces, hacer, utilizar, reusar o } \\
\text { comprar los componentes para el producto. }\end{array}$ \\
\hline 5 & $\begin{array}{l}\text { Programación } \\
\text { Integración }\end{array}$ & $\begin{array}{l}\text { * Implementar el diseño, elaborar la documentación necesaria, construcción e } \\
\text { integrar los diferentes componentes del producto, además de validar esta } \\
\text { integración. } \\
\text { * Realizar la preparación y ejecución de las pruebas del producto elaborado }\end{array}$ \\
\hline
\end{tabular}




\section{()}

\begin{tabular}{|c|c|c|}
\hline 6 & Certificación & $\begin{array}{l}\text { Realizar la validación del producto mediante la ejecución de pruebas de } \\
\text { certificación. }\end{array}$ \\
\hline 7 & Implementación & $\begin{array}{l}\text { Instalar el producto desarrollado en los ambientes del cliente para la ejecución } \\
\text { de pruebas finales. }\end{array}$ \\
\hline 8 & Cierre & $\begin{array}{l}\text { Documentar la evaluaciones y otros requisitos para gestionar el cierre del } \\
\text { proyecto en el sistema SARA }\end{array}$ \\
\hline 9 & Verificación & $\begin{array}{l}\text { Realizar la verificación de los entregables del proceso productivo de acuerdo a } \\
\text { criterios establecidos previamente. }\end{array}$ \\
\hline 10 & $\begin{array}{l}\text { Mantenimiento y } \\
\text { Asistencia Técnica }\end{array}$ & $\begin{array}{l}\text { Atender la cartera de clientes con necesidades de mantenimiento y asistencia } \\
\text { técnica, incluye la atención de reportes de problemas y mantenimientos que no } \\
\text { impliquen una modificación o ajuste en el producto. }\end{array}$ \\
\hline 11 & $\begin{array}{l}\text { Atención de } \\
\text { Requerimientos de } \\
\text { Desarrollo, Ajustes } \\
\text { y Modificaciones }\end{array}$ & $\begin{array}{l}\text { Gestionar la cartera de desarrollos, ajustes y modificaciones. Incluye la atención } \\
\text { de Reportes de problemas que implican un ajuste o modificación en el producto. }\end{array}$ \\
\hline $\mathbf{N}^{\mathbf{0}}$ & $\begin{array}{l}\text { Procesos } \\
\text { Estratégicos }\end{array}$ & OBJETIVO DEL PROCESO \\
\hline 12 & Planeamiento Anual & Realizar el plan anual de inversiones de la empresa. \\
\hline 13 & Revisión Gerencial & $\begin{array}{l}\text { Realizar seguimiento a los acuerdos plasmados en las reuniones de revisión } \\
\text { gerencial anuales. }\end{array}$ \\
\hline 14 & $\begin{array}{l}\text { Gestión de Riesgos } \\
\text { y Oportunidades }\end{array}$ & gos y oportunidades, que son de aplicación a la organización. \\
\hline $\mathbf{N}^{\mathbf{o}}$ & Proceso de Apoyo & OBJETIVO DEL PROCESO \\
\hline 15 & Logística & $\begin{array}{l}\text { Gestionar los recursos necesarios para todas las áreas de la empresa poniendo } \\
\text { especial énfasis en los recursos que impactan directamente en la calidad del } \\
\text { producto (servicios externos y otros proveedores) }\end{array}$ \\
\hline 16 & $\begin{array}{l}\text { Gestión de Talento } \\
\text { Humano }\end{array}$ & $\begin{array}{l}\text { Gestionar la capacitación interna y la contratación de personal idóneo para el } \\
\text { desempeño de los procesos. }\end{array}$ \\
\hline 17 & Soporte Interno & Brindar soporte interno a los usuarios de la empresa. \\
\hline 18 & $\begin{array}{l}\text { Administración de } \\
\text { la Configuración }\end{array}$ & $\begin{array}{l}\text { Mantener el orden, control de cambios y trazabilidad de los productos de la } \\
\text { empresa }\end{array}$ \\
\hline 19 & $\begin{array}{l}\text { Propiedad del } \\
\text { Cliente }\end{array}$ & Registrar y administrar la propiedad del cliente utilizada en los proyectos. \\
\hline 20 & $\begin{array}{l}\text { Aseguramiento de } \\
\text { la Calidad }\end{array}$ & Verificación documental de la línea base establecidas para los proyectos \\
\hline 21 & Mejora y Control de & Dar los lineamientos para la gestión de nuevos procesos en la empresa \\
\hline
\end{tabular}




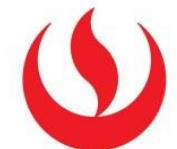

\begin{tabular}{|c|c|c|}
\hline & Procesos & \\
\hline 22 & $\begin{array}{lr}\text { Control de } & \text { No } \\
\text { Conformidades } & \text { y } \\
\text { Acciones } & \\
\text { Correctivas } & \end{array}$ & $\begin{array}{l}\text { Gestionar las no conformidades, potenciales no conformidades, así como las } \\
\text { acciones correctivas que ameriten. }\end{array}$ \\
\hline 23 & $\begin{array}{l}\text { Auditoría Interna y } \\
\text { Externa }\end{array}$ & $\begin{array}{l}\text { Gestionar las auditorías internas y externas de la empresa asi como las } \\
\text { observaciones o no conformidades originadas en las mismas. }\end{array}$ \\
\hline 24 & $\begin{array}{lr}\text { Control de } \\
\text { Documentos } \quad y \\
\text { Registros }\end{array}$ & $\begin{array}{l}\text { Establecer las pautas para la elaboración, modificación, publicación y control de } \\
\text { documentos y registros. }\end{array}$ \\
\hline 25 & Comunicaciones & $\begin{array}{l}\text { Gestión de la comunicación efectiva en la empresa, tanto de manera interna, } \\
\text { como hacia las partes interesadas. En este proceso se definirán los canales de } \\
\text { comunicación internos y externos establecidos por la empresa. } \\
\text { Mantener al personal interno/externo informado acerca de los asuntos y } \\
\text { decisiones relativas al funcionamiento del Sistema de Gestión de la Calidad. }\end{array}$ \\
\hline 26 & $\begin{array}{l}\text { Seguridad y Salud } \\
\text { en el Trabajo }\end{array}$ & $\begin{array}{l}\text { Mantener el Sistema de Gestión de Seguridad y Salud en el Trabajo en la } \\
\text { organización y cumplir con la normatividad nacional vigente aplicable en } \\
\text { materia de riesgos laborales. }\end{array}$ \\
\hline
\end{tabular}

Nota: Se ha descrito los objetivos de los procesos claves, estratégicos y de apoyo. Adaptado de "Manual de calidad”, por El Objeto de Estudio, 2017. 


\section{○ MATRIZ DE OBJETIVOS DEL NEGOCIO VS PROCESOS}

Los objetivos estratégicos son los objetivos planteados por una organización para así lograr determinadas metas. Al respecto, el Objeto de Estudio (2017) señala que:

1. Objetivo Estratégico 1: OE1: Contar con un índice de Satisfacción Global por Cliente en un 82\%. (Servicio integral: Soporte, Actividades, Proyectos).

2. Objetivo Estratégico 2: OE2: Lograr que el 90\% de personal del área de Operaciones tenga como mínimo 25 horas de capacitación orientadas al logro del incremento de la productividad.

3. Objetivo Estratégico 3: OE3: Crear por lo menos 2 nuevos productos competitivos durante el año, y actualizar 2 de los actuales.

4. Objetivo Estratégico 4: OE4: Invertir el 8\% de las ventas en la investigación y desarrollo tecnológico orientado a la productividad y competitividad.

5. Objetivo Estratégico 5: OE5: Incrementar en un 10\% la productividad mediante el mejoramiento de nuestros procesos (menos errores y velocidad de ejecución). (p. 16). 


\section{(}

Tabla 14

Matriz de Objetivos Estratégicos vs Procesos (ASIS)

\begin{tabular}{|c|c|c|c|c|c|c|c|c|c|c|c|c|c|c|c|c|c|c|c|c|c|c|c|c|c|c|c|}
\hline \multirow{3}{*}{$\begin{array}{l}\text { Objetivos } \\
\text { Estratégicos }\end{array}$} & \multicolumn{27}{|c|}{ PROCESOS } \\
\hline & \multicolumn{3}{|c|}{ Estratégicos } & \multicolumn{13}{|c|}{ Apoyo } & \multicolumn{11}{|c|}{ Claves } \\
\hline & 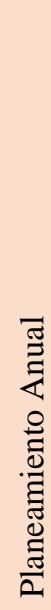 & 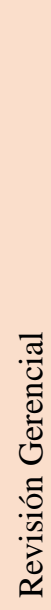 & 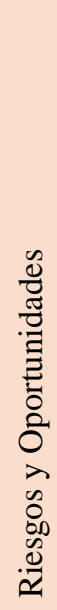 & 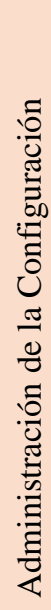 & 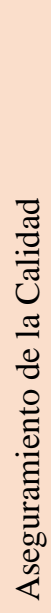 & 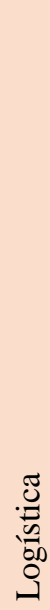 & 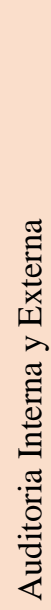 & 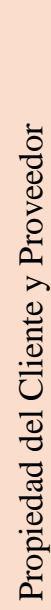 & 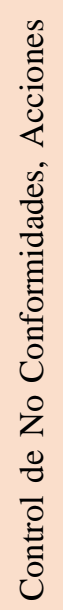 & 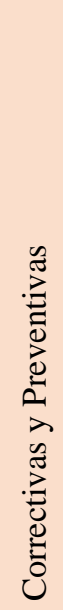 & 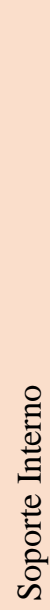 & $\begin{array}{l}0 \\
0 \\
0 \\
0 \\
0 \\
0 \\
2 \\
0 \\
0 \\
0 \\
0 \\
0 \\
0 \\
0 \\
0 \\
0 \\
0 \\
0 \\
0 \\
0\end{array}$ & 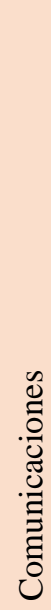 & $\begin{array}{l}0 \\
0 \\
0 \\
0 \\
0 \\
0 \\
0 \\
0 \\
0 \\
0 \\
0 \\
0 \\
\lambda \\
\frac{\pi}{0} \\
\frac{\pi}{2}\end{array}$ & 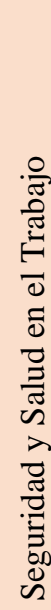 & 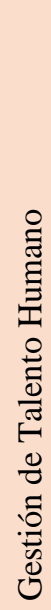 & 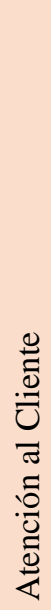 & $\begin{array}{l}0 \\
0 \\
0 \\
0 \\
0 \\
0 \\
0 \\
0 \\
0 \\
: 0 \\
0 \\
0 \\
0 \\
0\end{array}$ & 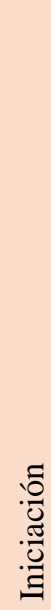 & 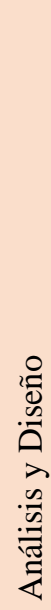 & 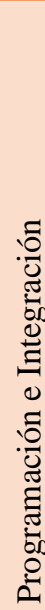 & 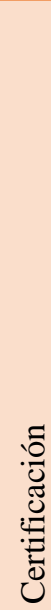 & 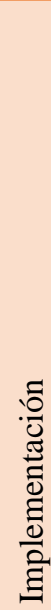 & $\stackrel{0}{\mathscr{U}}$ & 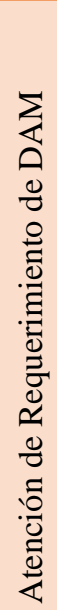 & 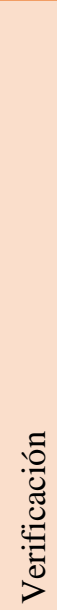 & $\sum_{\overline{0}}^{\frac{\pi}{0}}$ \\
\hline OE 1 & & $\mathrm{X}$ & & & & & & & & & & & & & & & $\mathrm{X}$ & $\mathrm{X}$ & & & & & & & & & \\
\hline OE 2 & $\mathrm{X}$ & & & & & & & & & & & & & & & $\mathrm{X}$ & & & & & & & & & & & \\
\hline OE 3 & & & & & & & & & & & & & & & & & & $\mathrm{X}$ & & & & & & & $\mathrm{X}$ & & \\
\hline OE 4 & $\mathrm{X}$ & $\mathrm{X}$ & & & & $X$ & & & & & & & & & & & & & & & & & & & & & \\
\hline OE 5 & $\mathrm{X}$ & & & & & & & & & & & & & & & & & $\mathrm{X}$ & $\mathrm{X}$ & $\mathrm{X}$ & $\mathrm{X}$ & $\mathrm{X}$ & $\mathrm{X}$ & $\mathrm{X}$ & $\mathrm{X}$ & $\mathrm{X}$ & $\mathrm{X}$ \\
\hline
\end{tabular}

Nota: Se ha realizado el cruce entre los objetivos estratégicos de la empresa y sus procesos para poder identificar cuales están relacionados. Adaptado de "Manual de

Calidad 2017”, por El Objeto de Estudio, 2017. 


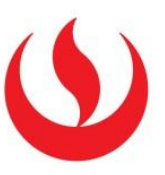

○ DIAGRAMA DE ACTTIVIDADES

○ SUBPROCESO DE “CONTROL Y SEGUIMIENTO DE PROYECTOS” (CSP)

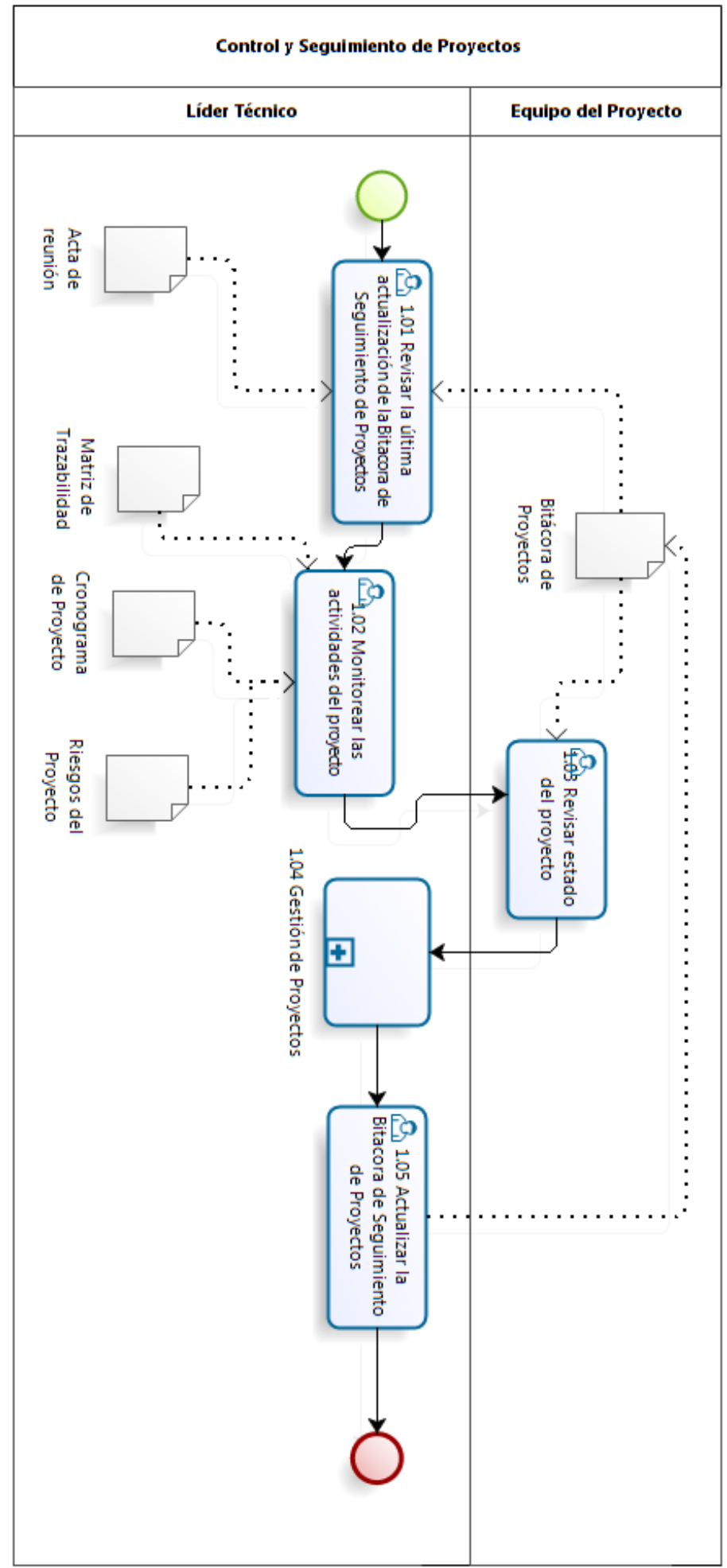

Figura 13. Diagrama de Actividades del Subproceso de Control y Seguimiento de Proyectos (ASIS). Adaptado de "Procedimiento de Gestión de Proyecto", por El Objeto de Estudio, 2017. 


\section{(1)}

○ SUBPROCESO DE “GESTIÓN DE PROYECTOS”

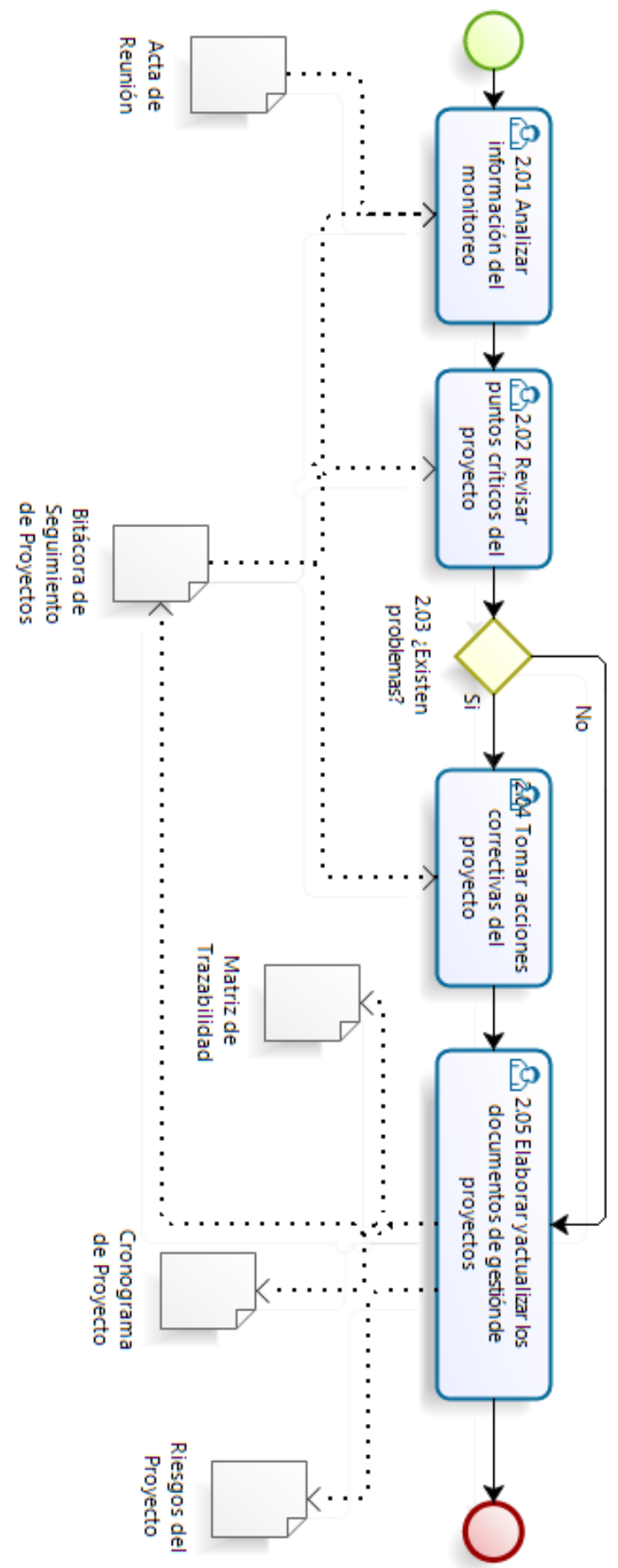

Figura 14. Diagrama de Actividades del Subproceso de Gestión de Proyectos (ASIS). Adaptado de "Procedimiento de Gestión de Proyecto", por El Objeto de Estudio, 2017. 


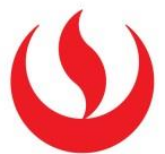

\section{PROCESO DE NEGOCIO SELECCIONADO Y DESCRIPCIÓN}

El proceso seleccionado es Gestión de Proyectos el cual gestiona los requerimientos a lo largo del ciclo de vida del proyecto, mantiene la trazabilidad de los requerimientos y administra cambios en los requerimientos. También, monitorea las actividades, compromisos, riesgos, involucrados directos e indirectos del proyecto. Asimismo toma las acciones correctivas de acuerdo al seguimiento que se realiza del proyecto. Identifica riesgos a lo largo del proyecto, define estrategias, evalúa, prioriza y desarrolla los planes de mitigación y/o contingencia para afrontar el riesgo.

Además, integra planes (actividades y recursos) coordina cruces y dependencias y realiza una coordinación integral. Por último, establece guías y criterios para tomar una decisión de acuerdo a las alternativas y métodos de evaluación definidos. Se divide en dos subprocesos:

\section{○ SUBPROCESO DE CSP10}

Tabla 15

Actividades del Subprocesos de Control y Seguimiento de Proyectos (ASIS)

\begin{tabular}{|c|c|c|c|}
\hline $\mathbf{N}^{\mathbf{o}}$ & ACTIVIDAD & RESPONSABLE & $\begin{array}{c}\text { REGISTRO/ } \\
\text { DOCUMENTO }\end{array}$ \\
\hline 1.01 & $\begin{array}{l}\text { Revisar la última actualización de la Bitácora de Seguimiento } \\
\text { de Proyectos } \\
\text { Revisar los acuerdos, no conformidades, problemas, lecciones } \\
\text { aprendidas y mejoras registrados en la bitácora de seguimiento de } \\
\text { proyectos encontrados en la última reunión de seguimiento del } \\
\text { proyecto (se entiende interna) y la externa con el cliente. } \\
\text { Cada uno de los puntos debe revisarse para conocer el estado } \\
\text { actual. } \\
\text { Periódicamente se deben realizar reuniones de seguimiento del } \\
\text { proyecto. }\end{array}$ & Líder técnico & $\begin{array}{l}\text { Acta de reunión / } \\
\text { Bitácora de } \\
\text { Seguimiento de } \\
\text { Proyectos }\end{array}$ \\
\hline 1.02 & $\begin{array}{l}\text { Monitorear las actividades del proyecto } \\
\text { - Los requerimientos del proyecto utilizando la Matriz de } \\
\text { Trazabilidad - a excepción de ATRs. } \\
\text { - Las variables del proyecto (plazo y esfuerzo), así como el avance }\end{array}$ & Líder técnico & $\begin{array}{l}\text { Matriz de } \\
\text { Trazabilidad }\end{array}$ \\
\hline
\end{tabular}

${ }^{10}$ Ver significado de CSP en la sección SIGLARIO 


\section{$(\varphi)$}

\begin{tabular}{|c|c|c|c|}
\hline \multirow[t]{2}{*}{$\mathbf{N}^{\mathbf{O}}$} & ACTIVIDAD & RESPONSABLE & $\begin{array}{c}\text { REGISTRO/ } \\
\text { DOCUMENTO }\end{array}$ \\
\hline & $\begin{array}{l}\text { de actividades (realizadas por personal interno o externo). Para } \\
\text { este monitoreo utilizamos el cronograma de proyecto. } \\
\text { - Los compromisos (hitos) del proyecto, para lo cual utilizamos el } \\
\text { cronograma de proyecto. } \\
\text { - Los riesgos del proyecto deben monitorearse utilizando la } \\
\text { plantilla Riesgos del Proyectos. } \\
\text { Las actividades del proyecto deben monitorearse de acuerdo a lo } \\
\text { registrado en el Sistema SARA. }\end{array}$ & & $\begin{array}{l}\text { Cronograma del } \\
\text { Proyecto } \\
\text { Riesgos del } \\
\text { Proyectos }\end{array}$ \\
\hline 1.03 & $\begin{array}{l}\text { Revisar el estado del proyecto } \\
\text { Revisar el estado de cada actividad, documento, entregable, riesgo, } \\
\text { compromiso, etc. Asimismo identificar todo problema o suceso } \\
\text { que pueda impactar el proyecto para poder ser reportado al } \\
\text { Gerente de Producto. } \\
\text { Nota: El equipo de trabajo debe transmitir sus inquietudes o sus } \\
\text { necesidades. }\end{array}$ & $\begin{array}{c}\text { Equipo de } \\
\text { Proyecto }\end{array}$ & $\begin{array}{l}\text { Bitácora de } \\
\text { Seguimiento de } \\
\text { Proyectos }\end{array}$ \\
\hline 1.04 & $\begin{array}{l}\text { Gestionar el proyecto } \\
\text { Realizar la Gestión del Proyecto utilizando la información } \\
\text { recolectada durante las actividades } 1.02 \text { y } 1.03 \text {. } \\
\text { Para ello debemos seguir el Proceso de Gestión de Proyectos. }\end{array}$ & Líder técnico & - \\
\hline 1.05 & $\begin{array}{l}\text { Actualizar la Bitácora de seguimiento de proyectos } \\
\text { Actualizar los acuerdos, mejoras, no conformidades, problemas, } \\
\text { lecciones aprendidas y mejoras en la Bitácora de seguimiento de } \\
\text { proyectos. } \\
\text { La bitácora de seguimiento de proyectos debe ser entregada al } \\
\text { Gerente de Producto para su revisión. }\end{array}$ & Líder técnico & $\begin{array}{l}\text { Bitácora de } \\
\text { Seguimiento de } \\
\text { Proyectos }\end{array}$ \\
\hline
\end{tabular}

Nota: Se ha descrito las actividades, responsable y registro/documento del subproceso de Control y Seguimiento de Proyectos. Adaptado de "Procedimiento de Gestión de Proyecto", por El Objeto de Estudio, 2017.

\section{○ SUBPROCESO DE GESTIÓN DE PROYECTO}

Tabla 16

Actividades del Subprocesos de Gestión de Proyectos (ASIS)

\begin{tabular}{|c|l|c|c|}
\hline $\mathbf{N}^{\mathbf{0}}$ & \multicolumn{1}{|c|}{ ACTIVIDAD } & RESPONSABLE & $\begin{array}{c}\text { REGISTRO/ } \\
\text { DOCUMENTO }\end{array}$ \\
\hline 2.01 & $\begin{array}{l}\text { Analizar la información obtenida durante la reunión de } \\
\text { seguimiento de proyecto. } \\
\text { Este análisis permitirá tomar las acciones correctivas necesarias. }\end{array}$ & Líder técnico & $\begin{array}{c}\text { Acta de reunión } \\
\text { / Bitácora de } \\
\text { Seguimiento de }\end{array}$ \\
\hline
\end{tabular}




\section{(4)}

\begin{tabular}{|c|c|c|c|}
\hline $\mathbf{N}^{\mathbf{0}}$ & ACTIVIDAD & RESPONSABLE & $\begin{array}{c}\text { REGISTRO/ } \\
\text { DOCUMENTO }\end{array}$ \\
\hline & $\begin{array}{l}\text { Nota: Los acuerdos, problemas, no conformidades (acción } \\
\text { correctiva), entre otros, se debe de colocar en la bitácora de } \\
\text { seguimiento de proyectos, en caso sea necesario se debe generar } \\
\text { un acta de la reunión. }\end{array}$ & & Proyectos \\
\hline 2.02 & $\begin{array}{l}\text { Revisar si el proyecto posee acciones correctivas pendientes de } \\
\text { cierre. Estas acciones correctivas pendientes de cierre deben } \\
\text { estar anotadas en la Bitácora de Seguimiento de Proyectos para } \\
\text { conocer el estado de avance de cada acción correctiva. }\end{array}$ & Líder técnico & $\begin{array}{l}\text { Bitácora de } \\
\text { Seguimiento de } \\
\text { Proyectos }\end{array}$ \\
\hline 2.03 & $\begin{array}{l}\text { ¿Existen problemas? } \\
\text { Si existen problemas dentro del proyecto se debe proceder a la } \\
\text { actividad 2.04. En caso contrario, realizar la actividad } 2.05 \text {. }\end{array}$ & Líder técnico & \\
\hline 2.04 & $\begin{array}{l}\text { Tomar acciones correctivas } \\
\text { Para ello en la Bitácora de seguimiento de Proyectos por cada } \\
\text { problema encontrado debemos: } \\
\text { - Anotar el problema suscitado. } \\
\text { - Anotar las acciones que se tomarán para su solución }\end{array}$ & Líder técnico & $\begin{array}{l}\text { Bitácora de } \\
\text { Seguimiento de } \\
\text { Proyectos }\end{array}$ \\
\hline 2.05 & $\begin{array}{l}\text { Debemos elaborar o actualizar los documentos de gestión del } \\
\text { proyecto, los cuales son: } \\
\text { - Matriz de Trazabilidad } \\
\text { - Cronograma de Proyecto } \\
\text { - Riesgos del Proyecto } \\
\text { - Bitácora de Seguimiento de Proyectos } \\
\text { Estos documentos son actualizados con la información } \\
\text { recolectada durante la reunión de seguimiento. Asimismo, son } \\
\text { presentados al Gerente de Producto para su revisión. } \\
\text { Nota: El Gerente de Producto de acuerdo a la información } \\
\text { revisada debe escalar los problemas y hechos que crea } \\
\text { conveniente tanto al Gerente de Operaciones como al Sponsor } \\
\text { del Proyecto. }\end{array}$ & Líder técnico & $\begin{array}{c}\text { Matriz de } \\
\text { Trazabilidad } \\
\text { Cronograma de } \\
\text { Proyecto } \\
\text { Riesgos del } \\
\text { Proyecto } \\
\\
\text { Bitácora de } \\
\text { Seguimiento de } \\
\text { Proyectos }\end{array}$ \\
\hline
\end{tabular}

Nota: Se ha descrito las actividades, responsable y registro/documento del subproceso de Gestión de Proyectos.

Adaptado de "Procedimiento de Gestión de Proyecto", por El Objeto de Estudio, 2017. 


\section{(4)}

○ ROLES DE NEGOCIO: MATRIZ RACI

- Subproceso de Control y Seguimiento de Proyectos

Tabla 17

Matriz RACI - Subproceso de CSP (ASIS)

\begin{tabular}{|c|c|c|c|c|c|}
\hline Matriz RACI & \multicolumn{5}{|c|}{ ROLES } \\
\hline $\begin{array}{ll}\text { R: Responsable } & \text { A: Aprobado } \\
\text { C: Consultado } & \text { I: Informado }\end{array}$ & $\begin{array}{l}\text { Gerente de } \\
\text { operaciones }\end{array}$ & $\begin{array}{l}\text { Asistente } \\
\text { de } \\
\text { Sistemas }\end{array}$ & $\begin{array}{l}\text { Gerente } \\
\text { de área }\end{array}$ & $\begin{array}{l}\text { Líder } \\
\text { técnico }\end{array}$ & $\begin{array}{c}\underline{\text { Equipo }} \\
\text { de } \\
\text { Proyecto }\end{array}$ \\
\hline \multicolumn{6}{|l|}{ Actividades } \\
\hline $\begin{array}{l}\text { Revisar la última actualización de la Bitácora de } \\
\text { Seguimiento de Proyectos. }\end{array}$ & I & $\mathrm{C}$ & I & $\mathrm{R}$ & $\mathrm{C}$ \\
\hline Monitorear las actividades del proyecto & I & $\mathrm{C}$ & I & $\mathrm{R}$ & $\mathrm{C}$ \\
\hline Revisar el estado del proyecto & I & $\mathrm{C}$ & I & $\mathrm{R}$ & $\mathrm{C}$ \\
\hline Gestionar el proyecto & I & $\mathrm{C}$ & I & $\mathrm{R}$ & $\mathrm{C}$ \\
\hline $\begin{array}{l}\text { Actualizar la Bitácora de seguimiento de } \\
\text { proyectos }\end{array}$ & I & $\mathrm{C}$ & I & $\mathrm{R}$ & $\mathrm{C}$ \\
\hline
\end{tabular}

Nota: Se ha descrito las actividades y roles del subproceso de Control y Seguimiento de Proyectos. Adaptado de “Procedimiento de Gestión de Proyecto", por El Objeto de Estudio, 2017.

\section{- Subproceso de Gestión de Proyectos}

Tabla 18

Matriz RACI - Subproceso de Gestión de Proyectos (ASIS)

\begin{tabular}{|c|c|c|c|c|c|}
\hline Matriz RACI & \multicolumn{5}{|c|}{ ROLES } \\
\hline $\begin{array}{ll}\text { R: Responsable } & \text { A: Aprobado } \\
\text { C: Consultado } & \text { I: Informado }\end{array}$ & $\begin{array}{l}\text { Gerente de } \\
\text { operaciones }\end{array}$ & $\begin{array}{l}\text { Asistente } \\
\text { de } \\
\text { Sistemas }\end{array}$ & $\begin{array}{l}\text { Gerente } \\
\text { de área }\end{array}$ & $\begin{array}{l}\text { Líder } \\
\text { técnico }\end{array}$ & $\begin{array}{c}\text { Equipo } \\
\text { de } \\
\text { Proyecto }\end{array}$ \\
\hline \multicolumn{6}{|l|}{ Actividades } \\
\hline $\begin{array}{l}\text { Analizar la información obtenida durante la } \\
\text { reunión de seguimiento de proyecto. }\end{array}$ & I & $\mathrm{C}$ & I & $\mathrm{R}$ & $\mathrm{C}$ \\
\hline $\begin{array}{l}\text { Revisar si el proyecto posee acciones correctivas } \\
\text { pendientes de cierre. }\end{array}$ & I & $\mathrm{C}$ & I & $\mathrm{R}$ & $\mathrm{C}$ \\
\hline Verificar si existen problemas & I & $\mathrm{C}$ & I & $\mathrm{R}$ & $\mathrm{C}$ \\
\hline Tomar acciones correctivas & A & $\mathrm{C}$ & A & $\mathrm{R}$ & $\mathrm{C}$ \\
\hline $\begin{array}{l}\text { Elaborar o actualizar los documentos de gestión } \\
\text { del proyecto }\end{array}$ & A & $\mathrm{C}$ & A & $\mathrm{R}$ & $\mathrm{C}$ \\
\hline
\end{tabular}

Nota: Se ha descrito las actividades y roles del subproceso de Gestión de Proyectos. Adaptado de

"Procedimiento de Gestión de Proyecto", por El Objeto de Estudio, 2017. 


\section{ARQUITECTURA DE DATOS}

○ MODELO DE DATOS

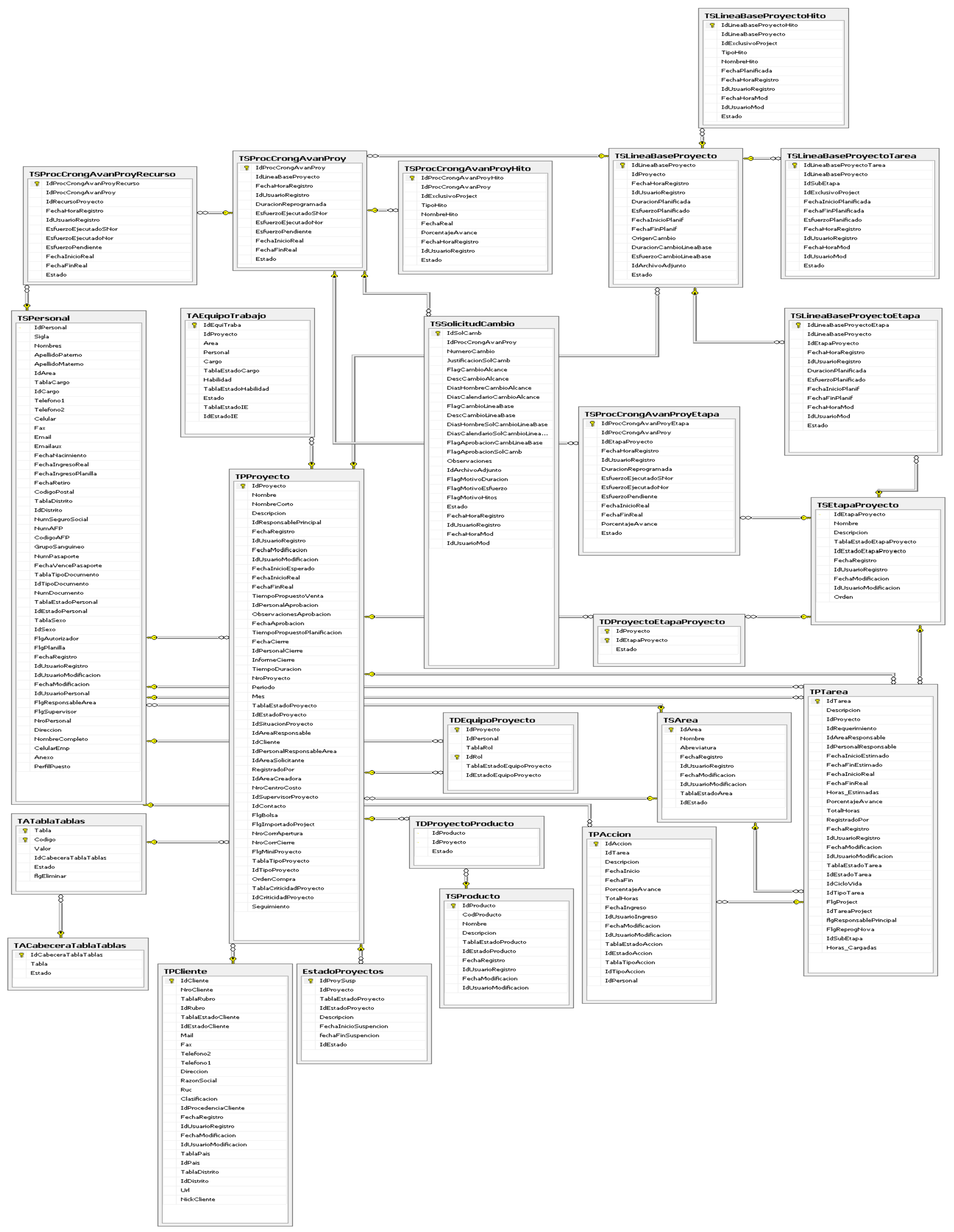

Figura 15. Modelo de Datos (ASIS). Adaptado de “Manual de Configuración”, por El Objeto de Estudio, 2017. 


\section{(4)}

○ DICCIONARIO DE DATOS

Tabla 19

Tabla TSLineaBaseProyecto

\begin{tabular}{|l|l|}
\hline TABLA & TSLineaBaseProyecto \\
\hline Descripción & Información sobre el proyecto línea base. \\
\hline ATRIBUTO & Descripción \\
\hline IdLineaBaseProyecto & Identificador del proyecto línea base \\
\hline IdProyecto & Identificador del proyecto \\
\hline DuraciónPlanificada & Duración planificada del proyecto \\
\hline EsfuerzoPlanificado & Esfuerzo planificado del proyecto \\
\hline FechaInicioPlanif & Fecha inicio planificada del proyecto \\
\hline FechaFinPlanif & Fecha fin planificada del proyecto \\
\hline OrigenCambio & Identificador del origen del cambio \\
\hline DuraciónCambioLineaBase & Duración del cambio de línea base \\
\hline EsfuerzoCambioLineaBase & Esfuerzo del cambio de línea base \\
\hline IdArchivoAdjunto & Archivo adjunto del cambio de línea base \\
\hline FechaHoraRegistro & Fecha que se realizó el registro \\
\hline IdUsuarioRegistro & Usuario que realizó el registro \\
\hline Estado & Estado del registro \\
\hline
\end{tabular}

Nota: Se ha descrito cada atributo de la tabla TSLineaBaseProyecto. Adaptado de "Manual de Configuración", por El Objeto de Estudio, 2017.

Tabla 20

Tabla TSLineaBaseProyectoHito

\begin{tabular}{|l|l|}
\hline TABLA & TSLineaBaseProyectoHito \\
\hline Descripción & Información sobre los hitos del proyecto línea base. \\
\hline ATRIBUTO & Descripción \\
\hline IdLineaBaseProyectoHito & Identificador de la tabla \\
\hline IdLineaBaseProyecto & Identificador del proyecto línea base \\
\hline IdExclusivoProject & Identificador de la tarea en el project \\
\hline TipoHito & Tipo de hito. \\
\hline NombreHito & Nombre del hito \\
\hline FechaPlanificada & Fecha planificada del hito \\
\hline FechaHoraRegistro & Fecha que se realizó el registro \\
\hline IdUsuarioRegistro & Usuario que realizó el registro \\
\hline FechaHoraMod & Fecha que se actualizó el registro \\
\hline IdUsuarioMod & Usuario que modificó el registro \\
\hline Estado & Estado del registro \\
\hline
\end{tabular}

Nota: Se ha descrito cada atributo de la tabla TSLineaBaseProyectoHito. Adaptado de "Manual de

Configuración”, por El Objeto de Estudio, 2017. 


\section{(}

Tabla 21

Tabla TSLineaBaseProyectoTarea

\begin{tabular}{|l|l|}
\hline TABLA & TSLineaBaseProyectoTarea \\
\hline Descripción & Información sobre las tareas del proyecto línea base. \\
\hline ATRIBUTO & Descripción \\
\hline IdLineaBaseProyectoTarea & Identificador de la tabla \\
\hline IdLineaBaseProyecto & Identificador del proyecto línea base \\
\hline IdSubEtapa & Identificador de la sub estapa del proyecto línea base \\
\hline IdExclusivoProject & Identificador de la tarea en el project \\
\hline FechaInicioPlanificada & Fecha inicio planificada de la tarea \\
\hline FechaFinPlanificada & Fecha fin planificada de la tarea \\
\hline EsfuerzoPlanificado & Esfuerzo planificado de la tarea \\
\hline FechaHoraRegistro & Fecha que se realizó el registro \\
\hline IdUsuarioRegistro & Usuario que realizó el registro \\
\hline FechaHoraMod & Fecha que se actualizó el registro \\
\hline IdUsuarioMod & Usuario que modificó el registro \\
\hline Estado & Estado del registro \\
\hline
\end{tabular}

Nota: Se ha descrito cada atributo de la tabla TSLineaBaseProyectoTarea. Adaptado de "Manual de

Configuración”, por El Objeto de Estudio, 2017.

\section{Tabla 22}

Tabla TSLineaBaseProyectoEtapa

\begin{tabular}{|l|l|}
\hline TABLA & TSLineaBaseProyectoEtapa \\
\hline Descripción & Información sobre las etapas del proyecto línea base. \\
\hline ATRIBUTO & Descripción \\
\hline IdLineaBaseProyectoEtapa & Identificador de la tabla \\
\hline IdLineaBaseProyecto & Identificador del proyecto línea base \\
\hline IdEtapaProyecto & Identificador de la etapa del proyecto \\
\hline DuraciónPlanificada & Duración planificada de la etapa \\
\hline EsfuerzoPlanificado & Esfuerzo planificado de la etapa \\
\hline FechaInicioPlanif & Fecha inicio planificada de la etapa \\
\hline FechaFinPlanif & Fecha fin planificada de la etapa \\
\hline FechaHoraRegistro & Fecha que se realizó el registro \\
\hline IdUsuarioRegistro & Usuario que realizó el registro \\
\hline FechaHoraMod & Fecha que se actualizó el registro \\
\hline IdUsuarioMod & Usuario que modificó el registro \\
\hline Estado & Estado del registro \\
\hline
\end{tabular}

Nota: Se ha descrito cada atributo de la tabla TSLineaBaseProyectoEtapa. Adaptado de "Manual de

Configuración”, por El Objeto de Estudio, 2017. 


\section{(9)}

Tabla 23

Tabla TDProyectoEtapaProyecto

\begin{tabular}{|l|l|}
\hline TABLA & TDProyectoEtapaProyecto \\
\hline Descripción & Información sobre las etapas de cada proyecto. \\
\hline ATRIBUTO & Descripción \\
\hline IdProyecto & Identificador del proyecto \\
\hline IdEtapaProyecto & Identificador de la etapa del proyecto \\
\hline Estado & Estado del registro \\
\hline
\end{tabular}

Nota: Se ha descrito cada atributo de la tabla TDProyectoEtapaProyecto. Adaptado de "Manual de

Configuración”, por El Objeto de Estudio, 2017.

Tabla 24

Tabla TSEtapaProyecto

\begin{tabular}{|l|l|}
\hline TABLA & TSEtapaProyecto \\
\hline Descripción & Información sobre las etapas del proyecto. \\
\hline ATRIBUTO & Descripción \\
\hline IdEtapaProyecto & Identificador de la etapa del proyecto línea base \\
\hline Nombre & Nombre de la etapa del proyecto \\
\hline Descripción & Descripción de la etapa del proyecto \\
\hline TablaEstadoEtapaProyecto & Tabla del estado de la etapa del proyecto \\
\hline IdEstadoEtapaProyecto & Identificador del estado de la etapa del proyecto \\
\hline FechaRegistro & Fecha que se realizó el registro \\
\hline IdUsuarioRegistro & Usuario que realizó el registro \\
\hline FechaModificacion & Fecha que se actualizó el registro \\
\hline IdUsuarioModificacion & Usuario que modificó el registro \\
\hline \multicolumn{1}{|c|}{ Orden } & \multicolumn{1}{c|}{ Orden de las etapas del proyecto } \\
\hline
\end{tabular}

Nota: Se ha descrito cada atributo de la tabla TSEtapaProyecto. Adaptado de "Manual de Configuración", por

El Objeto de Estudio, 2017.

Tabla 25

Tabla TSArea

\begin{tabular}{|l|l|}
\hline TABLA & TSArea \\
\hline Descripción & Información sobre las áreas. \\
\hline ATRIBUTO & Descripción \\
\hline IdArea & Identificador del área \\
\hline Nombre & Nombre del área \\
\hline Abreviatura & Abreviatura del área \\
\hline FechaRegistro & Fecha que se realizó el registro \\
\hline IdUsuarioRegistro & Usuario que realizó el registro \\
\hline FechaModificacion & Fecha que se actualizó el registro \\
\hline IdUsuarioModificacion & Usuario que modificó el registro \\
\hline
\end{tabular}




\section{(1)}

\begin{tabular}{|l|l|}
\hline TablaEstadoArea & Tabla del estado del área \\
\hline IdEstado & Estado del registro \\
\hline Nota: Se ha descrito cada atributo de la tabla TSArea. Adaptado de "Manual de Configuración”, por El Objeto
\end{tabular}

de Estudio, 2017.

Tabla 26

Tabla TDEquipoProyecto

\begin{tabular}{|l|l|}
\hline TABLA & TDEquipoProyecto \\
\hline Descripción & Información sobre los equipos de proyecto. \\
\hline ATRIBUTO & Descripción \\
\hline IdProyecto & Identificador del proyecto \\
\hline IdPersonal & Identificador del personal \\
\hline TablaRol & Tabla del rol \\
\hline IdRol & Identificador del rol \\
\hline TablaEstadoEquipoProyecto & Tabla del estado del equipo de proyecto \\
\hline IdEstadoEquipoProyecto & Estado del registro \\
\hline
\end{tabular}

Nota: Se ha descrito cada atributo de la tabla TDEquipoProyecto. Adaptado de "Manual de Configuración", por

El Objeto de Estudio, 2017.

Tabla 27

Tabla TSProducto

\begin{tabular}{|l|l|}
\hline TABLA & TSProducto \\
\hline Descripción & Información sobre el producto. \\
\hline ATRIBUTO & Descripción \\
\hline IdProducto & Identificador del producto \\
\hline CodProducto & Código del producto \\
\hline Nombre & Nombre del producto \\
\hline Descripción & Descripción del producto \\
\hline TablaEstadoProducto & Tabla de estado del producto \\
\hline IdEstadoProducto & Estado del registro \\
\hline FechaRegistro & Fecha que se realizó el registro \\
\hline IdUsuarioRegistro & Usuario que realizó el registro \\
\hline FechaModificacion & Fecha que se actualizó el registro \\
\hline IdUsuarioModificacion & Usuario que modificó el registro \\
\hline
\end{tabular}

Nota: Se ha descrito cada atributo de la tabla TSProducto. Adaptado de "Manual de Configuración", por El

Objeto de Estudio, 2017.

Tabla 28

Tabla TDProyectoProduto 


\section{(4)}

Descripción ATRIBUTO

IdProducto

IdProyecto

Estado
Información sobre la relación del producto con el proyecto.

\section{Descripción}

Identificador del producto

Identificador del proyecto

Estado del registro

Nota: Se ha descrito cada atributo de la tabla TDProyectoProduto. Adaptado de "Manual de Configuración", por

El Objeto de Estudio, 2017.

Tabla 29

Tabla TPTarea

\begin{tabular}{|c|c|}
\hline TABLA & TPTarea \\
\hline Descripción & Información sobre las tareas. \\
\hline ATRIBUTO & Descripción \\
\hline IdTarea & Identificador de la tarea \\
\hline Descripcion & Descripción de la tarea \\
\hline IdProyecto & Identificador del proyecto \\
\hline IdRequerimiento & Identificador del requerimiento \\
\hline IdAreaResponsable & Identificador del área responsable \\
\hline IdPersonaResponsable & Identificador del personal responsable \\
\hline FechaInicioEstimado & Fecha inicio estimado de la tarea \\
\hline FechaFinEstimado & Fecha fin estimado de la tarea \\
\hline FechaInicioReal & Fecha inicio real de la tarea \\
\hline FechaFinReal & Fecha fin real de la tarea \\
\hline Horas_Estimadas & Hora estimada de la tarea \\
\hline PorcentajeAvance & Porcentaje de avance de la tarea \\
\hline TotalHoras & Total de horas de la tarea \\
\hline RegistradoPor & Quien registra la tarea \\
\hline TablaEstadoTarea & Tabla de estado tarea \\
\hline IdEstadoTarea & Estado del registro \\
\hline IdCicloVida & Identificador del ciclo de vida \\
\hline IdTipoTarea & Identificador del tipo de acción \\
\hline FlgProject & Flag del project \\
\hline IdTareaProject & Identificador de la tarea en el project \\
\hline flgResponsablePrincipal & Flag del responsable principal \\
\hline FlgReprogNova & Falg de reprogramación de tarea \\
\hline IdSubEtapa & Identidificador de subtarea \\
\hline Horas_Cargadas & Horas cargadas de la tarea \\
\hline FechaRegistro & Fecha que se realizó el registro \\
\hline IdUsuarioRegistro & Usuario que realizó el registro \\
\hline FechaModificacion & Fecha que se actualizó el registro \\
\hline IdUsuarioModificacion & Usuario que modificó el registro \\
\hline
\end{tabular}

Nota: Se ha descrito cada atributo de la tabla TPTarea. Adaptado de "Manual de Configuración", por El Objeto

de Estudio, 2017. 


\section{(9)}

Tabla 30

Tabla TPAccion

\begin{tabular}{|l|l|}
\hline TABLA & TPAccion \\
\hline Descripción & Información sobre las acciones de la tarea. \\
\hline ATRIBUTO & Descripción \\
\hline IdAccion & Identificador de acción \\
\hline IdTarea & Identificador de la tarea \\
\hline Descripcion & Descripción de la acción \\
\hline FechaInicio & Fecha inicio de la acción \\
\hline FechaFin & Fecha fin de la acción \\
\hline PorcentajeAvance & Porcentaje de avance de la acción \\
\hline TotalHoras & Total de horas de la acción \\
\hline TablaEstadoAccion & Tabla de estado acción \\
\hline IdEstadoAccion & Estado del registro \\
\hline TablaTipoAccion & Tabla tipo de acción \\
\hline IdTipoAccion & Identificador del tipo de acción \\
\hline IdPersonal & Identificador del personal \\
\hline FechaIngreso & Fecha que se realizó el registro \\
\hline IdUsuarioIngreso & Usuario que realizó el registro \\
\hline FechaModificacion & Fecha que se actualizó el registro \\
\hline IdUsuarioModificacion & Usuario que modificó el registro \\
\hline
\end{tabular}

Nota: Se ha descrito cada atributo de la tabla TPAccion. Adaptado de "Manual de Configuración", por El

Objeto de Estudio, 2017.

Tabla 31

Tabla TPCliente

\begin{tabular}{|l|l|}
\hline TABLA & TPCliente \\
\hline Descripción & Información sobre los clientes. \\
\hline ATRIBUTO & Descripción \\
\hline IdCliente & Identificador del cliente \\
\hline NroCliente & Número del cliente \\
\hline TablaRubro & Tabla de rubro \\
\hline IdRubro & Identificador de rubro \\
\hline TablaEstadoCliente & Tabla estado del cliente \\
\hline IdEstadoCliente & Estado del registro \\
\hline Mail & Mail del cliente \\
\hline Fax & Fax del cliente \\
\hline Telefono2 & Teléfono 2 del cliente \\
\hline Telefono1 & Teléfono 1 del cliente \\
\hline Dirección & Dirección del cliente \\
\hline RazonSocial & Razon Social del cliente \\
\hline Ruc & Ruc del cliente \\
\hline
\end{tabular}




\section{(4)}

\begin{tabular}{|l|l|}
\hline Clasificación & Clasificación del cliente \\
\hline IdProcedenciaCliente & Identificador de procedencia del cliente \\
\hline TablaPais & Tabla de Pais \\
\hline IdPais & Identificador del país \\
\hline TablaDistrito & Tabla de Distrito \\
\hline IdDistrito & Identificador del distrito \\
\hline Url & Url \\
\hline NickCliente & Nick del cliente \\
\hline FechaRegistro & Fecha que se realizó el registro \\
\hline IdUsuarioRegistro & Usuario que realizó el registro \\
\hline FechaModificacion & Fecha que se actualizó el registro \\
\hline IdUsuarioModificacion & Usuario que modificó el registro \\
\hline
\end{tabular}

Nota: Se ha descrito cada atributo de la tabla TPCliente. Adaptado de "Manual de Configuración", por El

Objeto de Estudio, 2017.

Tabla 32

Tabla EstadoProyectos

\begin{tabular}{|l|l|}
\hline TABLA & EstadoProyectos \\
\hline Descripción & Información sobre los estados de los proyectos. \\
\hline ATRIBUTO & Descripción \\
\hline IdProySusp & Identificador del proyecto suspendido \\
\hline IdProyecto & Identificador del proyecto \\
\hline TablaEstadoProyecto & Tabla estado del proyecto \\
\hline IdEstadoProyecto & Estado del registro \\
\hline Descripción & Descripción del estado del proyecto \\
\hline FechaInicioSuspención & Fecha inicio de suspensión del proyecto \\
\hline FechaFinSuspención & Fecha fin de suspensión del proyecto \\
\hline IdEstado & Estado del registro \\
\hline
\end{tabular}

Nota: Se ha descrito cada atributo de la tabla EstadoProyectos. Adaptado de "Manual de Configuración", por El

Objeto de Estudio, 2017.

Tabla 33

Tabla TATablaTablas

\begin{tabular}{|l|l|}
\hline TABLA & TATablaTablas \\
\hline Descripción & Información sobre la tabla de tablas. \\
\hline ATRIBUTO & Descripción \\
\hline Tabla & Identificador de la tabla de tablas \\
\hline Codigo & Identificador d de la tabla de tablas \\
\hline Valor & Valor de la tabla de tablas \\
\hline IdCabeceraTablaTablas & Identificador de la cabecera de la tabla de tablas \\
\hline Estado & Estado del registro \\
\hline
\end{tabular}




\section{(4)}

flgEliminar

Flag de eliminación

Nota: Se ha descrito cada atributo de la tabla TATablaTablas. Adaptado de "Manual de Configuración", por El

Objeto de Estudio, 2017.

Tabla 34

Tabla TACabeceraTablaTablas

\begin{tabular}{|l|l|}
\hline TABLA & TACabeceraTablaTablas \\
\hline Descripción & Información sobre la cabecera de tabla de tablas. \\
\hline ATRIBUTO & Descripción \\
\hline IdCabeceraTablaTablas & Identificador de la cabecera de la tabla de tablas \\
\hline Tabla & La tabla \\
\hline Estado & Estado del registro \\
\hline
\end{tabular}

Nota: Se ha descrito cada atributo de la tabla TACabeceraTablaTablas. Adaptado de "Manual de

Configuración”, por El Objeto de Estudio, 2017.

Tabla 35

Tabla TPProyecto

\begin{tabular}{|l|l|}
\hline TABLA & TPProyecto \\
\hline Descripción & Información sobre los proyectos. \\
\hline ATRIBUTO & Descripción \\
\hline IdProyecto & Identificador del proyecto \\
\hline Nombre & Nombre del proyecto \\
\hline NombreCorto & Nombre corto del proyecto \\
\hline Descripción & Descripción del proyecto \\
\hline IdResponsablePrinciapl & Identificador del Responsable Principal \\
\hline FechaInicioEsperado & Fecha inicio esperado del proyecto \\
\hline FechaInicioReal & Fecha inicio real del proyecto \\
\hline FechaFinReal & Fecha fin real del proyecto \\
\hline TiempoPropuestoVenta & Tiempo de propuesta de venta \\
\hline IdPersonalAprobación & Identificador del personal de aprobación \\
\hline ObservacionesAprobación & Observaciones de la aprobación \\
\hline FechaAprobación & Fecha de aprobación \\
\hline TiempoPropuestoPlanificació & Tiempo propuesto de planificación \\
\hline n & Fecha de cierre \\
\hline FechaCierre & Identificador del personal cierre \\
\hline IdPersonalCierre & Informe de cierre \\
\hline InformeCierre & Tiempo de duración \\
\hline TiempoDuración & Número del proyecto \\
\hline NroProyecto & Periodo del proyecto \\
\hline Periodo & Mes del proyecto \\
\hline Mes & Tabla del estado proyecto \\
\hline TablaEstadoProyecto & \\
\hline
\end{tabular}




\section{(4)}

\begin{tabular}{|l|l|}
\hline IdEstadoProyecto & Estado del registro \\
\hline IdSituacionProyecto & Identificador de la situación del proyecto \\
\hline IdAreaResponsable & Identificador del área responsable \\
\hline IdCliente & Identificador del cliente \\
\hline IdPersonalResponsableArea & Identificador del Personal Responsable del área \\
\hline IdAreaSolicitante & Identificador del área solicitante \\
\hline Registrado por & Quien registra el proyecto \\
\hline IdAreaCreadora & Identificador del área creadora \\
\hline NroCentroCosto & Numero de centro de costo \\
\hline IdSupervisorProyecto & Identificador del supervisor del proyecto \\
\hline IdContacto & Identificador del contacto \\
\hline FlgBolsa & Flag de Bolsa \\
\hline FlgImportadoProject & Flag de importación del Project \\
\hline NroCorrApertura & Numero correlativo de apertura \\
\hline NroCorrCierre & Numero correlativo de cierre \\
\hline FlagMiniProyecto & Flag de mini proyecto \\
\hline TablaTipoProyecto & Tabla de tipo de proyecto \\
\hline IdTipoProyecto & Identificador de tipo de proyecto \\
\hline OrdenCompra & Orden de compra \\
\hline TablaCriticidadProyecto & Tabla de criticidad de proyecto \\
\hline IdCriticidadProyecto & Identificador de criticidad de proyecto \\
\hline Seguimiento & Seguimiento de proyecto \\
\hline FechaRegistro & Fecha que se realizó el registro \\
\hline IdUsuarioRegistro & Usuario que realizó el registro \\
\hline FechaModificacion & Fecha que se actualizó el registro \\
\hline IdUsuarioModificacion & Usuario que modificó el registro \\
\hline
\end{tabular}

Nota: Se ha descrito cada atributo de la tabla TPProyecto. Adaptado de "Manual de Configuración", por El

Objeto de Estudio, 2017.

Tabla 36

Tabla TAEquipoTrabajo

\begin{tabular}{|l|l|}
\hline TABLA & TAEquipoTrabajo \\
\hline Descripción & Información sobre el equipo de trabajo. \\
\hline ATRIBUTO & Descripción \\
\hline IdEquiTraba & Identificador de equipo de trabajo \\
\hline IdProyecto & Identificador del proyecto \\
\hline Area & Área que pertenece \\
\hline Personal & Personal \\
\hline Cargo & Cargo al que pertenece \\
\hline TablaEstadoCargo & Tabla estado de cargo \\
\hline Habilidad & Habilidad del equipo de trabajo \\
\hline TablaEstadoHabilidad & Tabla de estado de habilidad \\
\hline Estado & Estado del registro \\
\hline
\end{tabular}




\section{(4)}

TablaEstadoIE

IdestadoIE

Tabla de estado IE

Estado del registro

Nota: Se ha descrito cada atributo de la tabla TAEquipoTrabajo. Adaptado de "Manual de Configuración", por

El Objeto de Estudio, 2017.

Tabla 37

Tabla TSPersonal

\begin{tabular}{|l|l|}
\hline TABLA & TSPersonal \\
\hline Descripción & Información sobre el personal. \\
\hline ATRIBUTO & Descripción \\
\hline IdPersonal & Identificador de la persona \\
\hline Sigla & Sigla de la persona \\
\hline Nombres & Nombre de la persona \\
\hline ApellidoPaterno & Apellido Paterno de la persona \\
\hline ApellidoMaterno & Apellido Materno de la persona \\
\hline IdArea & Identificador del área \\
\hline TablaCargo & Tabla de cargo \\
\hline IdCargo & Identificador del cargo \\
\hline Telefono1 & Teléfono 1 de la persona \\
\hline Telefono2 & Teléfono 2 de la persona \\
\hline Celular & Celular de la persona \\
\hline Fax & Fax de la persona \\
\hline Email & Email de la persona \\
\hline Emailaux & Email auxiliar de la persona \\
\hline FechaNacimiento & Fecha de nacimiento de la persona \\
\hline FechaIngresoReal & Fecha de Ingreso real de la persona \\
\hline FechaIngresoPlanilla & Fecha de ingreso a planilla de la persona \\
\hline FechaRetiro & Fecha de retiro de la persona \\
\hline CodigoPostal & Còdigo postal \\
\hline TablaDistrito & Tabla de Distrito \\
\hline IdDistrito & Identificador del distrito \\
\hline NumSeguroSocial & Número de seguro social \\
\hline NumAFP & Numero de AFP \\
\hline CodigoAFP & Código de AFP \\
\hline GrupoSanguineo & Grupo Sanguíneo de la persona \\
\hline NumPasaporte & Numero de pasaporte \\
\hline FechaVencePasaporte & Fecha de vencimiento del pasaporte \\
\hline TablaTipoDocumento & Tabla de tipo de documento \\
\hline IdTipoDocumento & Identificador del tipo de documento \\
\hline NumDocumento & Numero de documento \\
\hline TablaEstadoPersonal & Tabla estado de personal \\
\hline IdEstadoPersonal & Estado del registro \\
\hline TablaSexo & Tabla de sexo de la persona \\
\hline & \\
\hline
\end{tabular}




\section{(4)}

\begin{tabular}{|l|l|}
\hline IdSexo & Identificador del sexo de la persona \\
\hline FlgAutorizador & Flag autorizador \\
\hline FlgPlanilla & Flag de planilla \\
\hline IdUsuarioPersonal & Identificador del usuario de la persona \\
\hline FlgResponsableArea & Flag del responsable del área \\
\hline FlgSupervisor & Flag supervisor \\
\hline NroPersonal & Número de la persona \\
\hline Direcciòn & Dirección de la persona \\
\hline NombreCompleto & Nombre completo de la persona \\
\hline CelularEmp & Celular de la empresa de la persona \\
\hline Anexo & Anexo de la persona \\
\hline PerfilPuesto & Perfil del puesto de la persona \\
\hline FechaRegistro & Fecha que se realizó el registro \\
\hline IdUsuarioRegistro & Usuario que realizó el registro \\
\hline FechaModificacion & Fecha que se actualizó el registro \\
\hline IdUsuarioModificacion & Usuario que modificó el registro \\
\hline
\end{tabular}

Nota: Se ha descrito cada atributo de la tabla TSPersonal. Adaptado de "Manual de Configuración", por El

Objeto de Estudio, 2017.

Tabla 38

Tabla TSProcCrongAvanProy

\begin{tabular}{|l|l|}
\hline TABLA & TSProcCrongAvanProy \\
\hline Descripción & $\begin{array}{l}\text { Información sobre el avance del procesamiento del } \\
\text { cronograma del proyecto. }\end{array}$ \\
\hline ATRIBUTO & Descripción \\
\hline IdProcCrongAvanProy & Identificador del procesamiento del cronograma del proyecto \\
\hline IdLineaBaseProyecto & Identificador de la línea base del proyecto \\
\hline FechaHoraRegistro & Fecha que se realizó el registro \\
\hline IdUsuarioRegistro & Usuario que realizó el registro \\
\hline DuraciónReprogramada & Duración Reprogramada \\
\hline EsfuerzoEjecutadoSNor & Esfuerzo ejecutado sin normalizar \\
\hline EsfuerzoEjecutadoNor & Esfuerzo ejecutado normalizado \\
\hline EsfuerzoPendiente & Esfuerzo pendiente \\
\hline FechaInicioReal & Fecha inicio real \\
\hline FechaFinReal & Fecha fin real \\
\hline Estado & Estado del registro \\
\hline
\end{tabular}

Nota: Se ha descrito cada atributo de la tabla TSProcCrongAvanProy. Adaptado de "Manual de Configuración", por El Objeto de Estudio, 2017.

Tabla 39

Tabla TSProcCrongAvanProyRecurso 


\section{(4)}

\begin{tabular}{|l|l|}
\hline Descripción & $\begin{array}{l}\text { Información sobre el recurso en el avance del procesamiento } \\
\text { del cronograma del proyecto. } \\
\text { Descripción }\end{array}$ \\
\hline $\begin{array}{l}\text { ATRIBUTO } \\
\text { IdProcCrongAvanProyRecurs } \\
\text { o }\end{array}$ & $\begin{array}{l}\text { Identificador del recurso en el procesamiento del } \\
\text { cronograma del proyecto }\end{array}$ \\
\hline IdProcCrongAvanProy & Identificador del procesamiento del cronograma del proyecto \\
\hline IdRecursoProyecto & Identificador del recurso del proyecto \\
\hline FechaHoraRegistro & Fecha que se realizó el registro \\
\hline IdUsuarioRegistro & Usuario que realizó el registro \\
\hline EsfuerzoEjecutadoSNor & Esfuerzo ejecutado sin normalizar \\
\hline EsfuerzoEjecutadoNor & Esfuerzo ejecutado normalizado \\
\hline EsfuerzoPendiente & Esfuerzo pendiente \\
\hline FechaInicioReal & Fecha inicio real \\
\hline FechaFinReal & Fecha fin real \\
\hline Estado & Estado del registro \\
\hline
\end{tabular}

Nota: Se ha descrito cada atributo de la tabla TSProcCrongAvanProyRecurso. Adaptado de "Manual de

Configuración”, por El Objeto de Estudio, 2017.

Tabla 40

Tabla TSProcCrongAvanProyHito

\begin{tabular}{|l|l|}
\hline TABLA & TSProcCrongAvanProyHito \\
\hline Descripción & $\begin{array}{l}\text { Información sobre el hito en el avance del procesamiento del } \\
\text { cronograma del proyecto. } \\
\text { Descripción }\end{array}$ \\
\hline ATRIBUTO & $\begin{array}{l}\text { Identificador del hito en el procesamiento del cronograma } \\
\text { del proyecto }\end{array}$ \\
\hline IdProcCrongAvanProyHito & Identificador del procesamiento del cronograma del proyecto \\
\hline IdProcCrongAvanProy & Identificador exclusivo de la tarea en el project \\
\hline IdExclusivoProject & Tipo de hito \\
\hline TipoHito & Nombre del hito \\
\hline NombreHito & Fecha real \\
\hline FechaReal & Porcentaje de avance \\
\hline ProcentajeAvance & Fecha que se realizó el registro \\
\hline FechaHoraRegistro & Usuario que realizó el registro \\
\hline IdUsuarioRegistro & Estado del registro \\
\hline Estado &
\end{tabular}

Nota: Se ha descrito cada atributo de la tabla TSProcCrongAvanProyHito. Adaptado de "Manual de

Configuración”, por El Objeto de Estudio, 2017.

Tabla 41

Tabla TSProcCrongAvanProyEtapa

TABLA

Descripción
TSProcCrongAvanProyEtapa

Información sobre la etapa en el avance del procesamiento del 


\section{(4)}

\begin{tabular}{|l|l|}
\hline ATRIBUTO & cronograma del proyecto. \\
\hline IdProcCrongAvanProyEtapa & $\begin{array}{l}\text { Descripción } \\
\text { Identificador de la etapa en el procesamiento del cronograma } \\
\text { del proyecto }\end{array}$ \\
\hline IdProcCrongAvanProy & Identificador del procesamiento del cronograma del proyecto \\
\hline IdEtapaProyecto & Identificador de la etapa del proyecto \\
\hline FechaHoraRegistro & Fecha que se realizó el registro \\
\hline IdUsuarioRegistro & Usuario que realizó el registro \\
\hline DuraciónReprogramada & Duración Reprogramada \\
\hline EsfuerzoEjecutadoSNor & Esfuerzo ejecutado sin normalizar \\
\hline EsfuerzoEjecutadoNor & Esfuerzo ejecutado normalizado \\
\hline EsfuerzoPendiente & Esfuerzo pendiente \\
\hline FechaInicioReal & Fecha inicio real \\
\hline FechaFinReal & Fecha fin real \\
\hline PorcentajeAvance & Porcentaje de avance \\
\hline Estado & Estado del registro \\
\hline
\end{tabular}

Nota: Se ha descrito cada atributo de la tabla TSProcCrongAvanProyEtapa. Adaptado de "Manual de

Configuración”, por El Objeto de Estudio, 2017.

Tabla 42

Tabla TSSolicitudCambio

\begin{tabular}{|c|c|}
\hline TABLA & TSSolicitudCambio \\
\hline Descripción & $\begin{array}{l}\text { Información sobre la solicitud de cambio del } \\
\text { proyecto. }\end{array}$ \\
\hline ATRIBUTO & Descripción \\
\hline IdSolCamb & Identificador de la solicitud de cambio \\
\hline IdProcCrongAvanProy & $\begin{array}{l}\text { Identificador del procesamiento del cronograma del } \\
\text { proyecto }\end{array}$ \\
\hline NumeroCambio & Número de la solicitud de cambio \\
\hline JustificacionSolCamb & Justificación de la solicitud de cambio \\
\hline FlagCambioAlcance & Flag del cambio de alcance \\
\hline DescCambioAlcance & Descripción del cambio de alcance \\
\hline DiasHombreCambioAlcance & Días hombre del cambio de alcance \\
\hline DiasCalendarioCambioAlcance & Días calendario del cambio de alcance \\
\hline FlagCambioLineaBase & Flag del cambio de línea base \\
\hline DescCambioLineaBase & Descripción del cambio de línea base \\
\hline DiasHombreSolCambioLineaBase & Días hombre del cambio de línea base \\
\hline DiasCalendarioSolCambioLineaBase & Días calendario del cambio de línea base \\
\hline FlagAprobaciónCambLineaBase & Flag de aprobación del cambio de línea base \\
\hline FlagAprobaciónSolCamb & Flag de aprobación de la solicitud de cambio \\
\hline Observaciones & Observaciones de la solicitud de cambio \\
\hline IdArchivoAdjunto & Identificador del archivo adjunto \\
\hline FlagMotivoDuración & Flag del motivo de duración \\
\hline
\end{tabular}




\begin{tabular}{|l|l|}
\hline FlagMotivoEsfuerzo & Flag del motivo de esfuerzo \\
\hline FlagMotivoHitos & Flag del motivo de hitos \\
\hline Estado & Estado del registro \\
\hline FechaHoraRegistro & Fecha que se realizó el registro \\
\hline IdUsuarioRegistro & Usuario que realizó el registro \\
\hline FechaHoraMod & Fecha que se modificó el registro \\
\hline IdUsuarioMod & Usuario que modificó el registro \\
\hline
\end{tabular}

Nota: Se ha descrito cada atributo de la tabla TSSolicitudCambio. Adaptado de "Manual de Configuración", por El Objeto de Estudio, 2017. 


\section{(1)}

\section{○ MATRIZ DE ENTIDADES DE DATOS VS PROCESOS DEL NEGOCIO}

Tabla 43

Matriz de Entidades de Datos vs Procesos del Negocio (ASIS)

\begin{tabular}{|c|c|c|c|c|c|c|c|c|c|c|c|c|c|c|c|c|c|c|c|c|c|c|c|c|c|c|c|}
\hline \multirow[t]{3}{*}{ Entidades de Datos } & \multicolumn{27}{|c|}{ PROCESOS } \\
\hline & \multicolumn{3}{|c|}{ Estratégicos } & \multicolumn{13}{|c|}{ Apoyo } & \multicolumn{11}{|c|}{ Claves } \\
\hline & 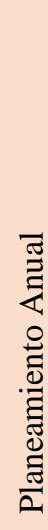 & 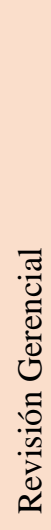 & 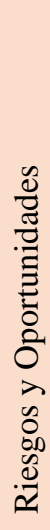 & 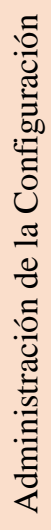 & 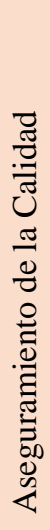 & 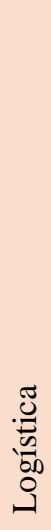 & 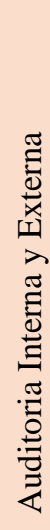 & 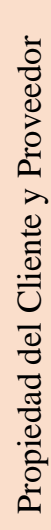 & 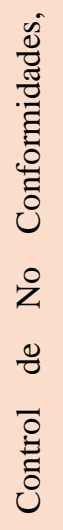 & 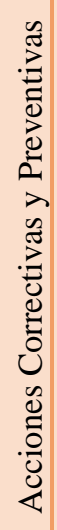 & 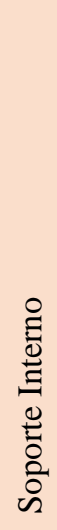 & 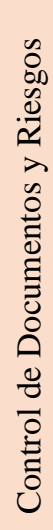 & 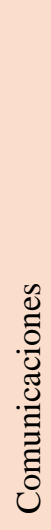 & 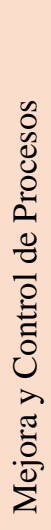 & 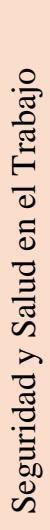 & 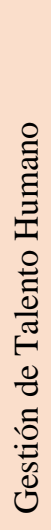 & 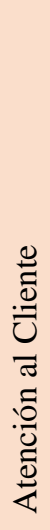 & 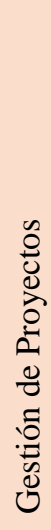 & 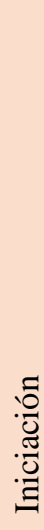 & 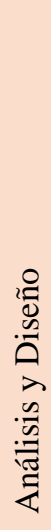 & 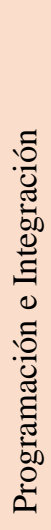 & 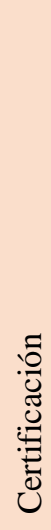 & 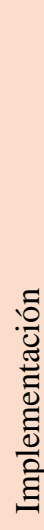 & تِّ & 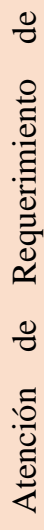 & 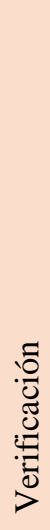 & 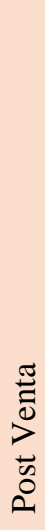 \\
\hline TSETAPAPROYECTO & & & & & & & & & & & & & & & & & & $X$ & & & & & & & & & \\
\hline TPCLIENTE & & & & & & & & & & & & & & & & & & $X$ & & & & & & & & & \\
\hline $\begin{array}{l}\text { TSPROCCRONGAVANPROY } \\
\text { ETAPA }\end{array}$ & & & & & & & & & & & & & & & & & & $X$ & & & & & & & & & \\
\hline $\begin{array}{l}\text { TDPROYECTOETAPAPROY } \\
\text { ECTO }\end{array}$ & & & & & & & & & & & & & & & & & & $X$ & & & & & & & & & \\
\hline TPACCION & & & & & & & & & & & & & & & & & & $X$ & & & & & & & & & \\
\hline $\begin{array}{l}\text { TSLINEABASEPROYECTOE } \\
\text { TAPA }\end{array}$ & & & & & & & & & & & & & & & & & & $X$ & & & & & & & & & \\
\hline TSLINEABASEPROYECTOH & & & & & & & & & & & & & & & & & & $\mathrm{X}$ & & & & & & & & & \\
\hline
\end{tabular}




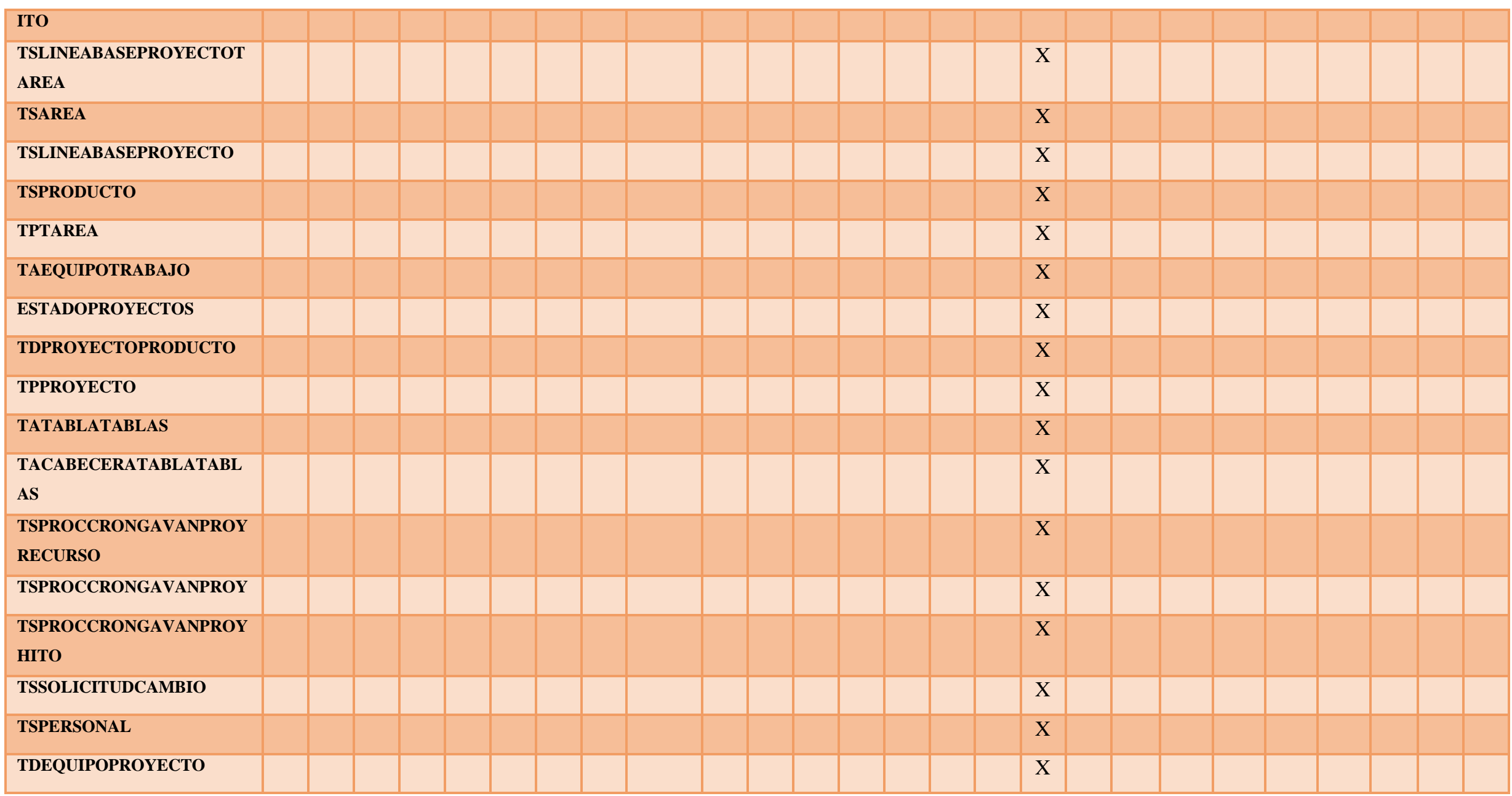

Nota: Se ha realizado el cruce entre tablas y procesos para poder identificar cuales están relacionados. Adaptado de "Manual de Configuración”, por El Objeto de Estudio, 2017. 


\section{ARQUITECTURA DE APLICACIÓN}

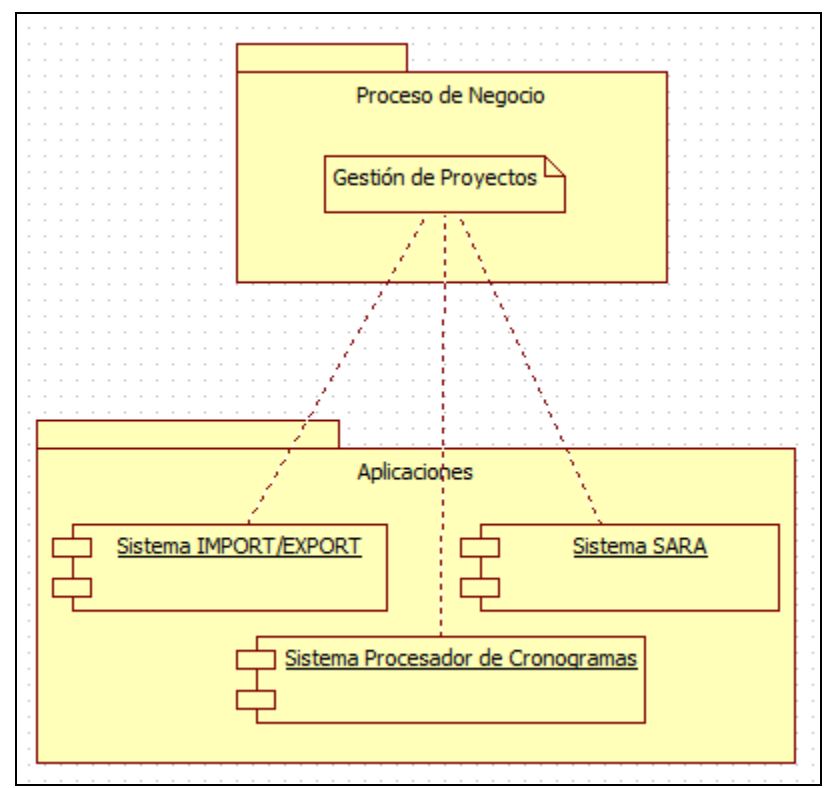

Figura 16. Diagrama de Aplicaciones del Proceso de Gestión de Proyectos (ASIS). Adaptado de "Manual de Configuración”, por El Objeto de Estudio, 2017.

Tabla 44

Detalle de Aplicaciones del Proceso Gestión de Proyectos (ASIS)

\begin{tabular}{|l|l|l|}
\hline Proceso de Negocio & Aplicación & Descripción \\
\hline $\begin{array}{l}\text { Gestión } \\
\text { Proyectos }\end{array}$ & $\begin{array}{l}\text { Sistema } \\
\text { IMPORT/EXPORT }\end{array}$ & $\begin{array}{l}\text { Se encarga de sincronizar las actividades del } \\
\text { cronograma con el Sistema SARA (Importación), el } \\
\text { sistema deberá leer un archivo Project y exportarlo } \\
\text { con información actualizada del sistema SARA } \\
\text { (exportación). }\end{array}$ \\
\hline & Sistema SARA & $\begin{array}{l}\text { Contiene información sobre las áreas, los productos, } \\
\text { los proyectos, y trabajadores de la empresa, las } \\
\text { actividades de proyectos solo las ingresan el personal } \\
\text { del área de Operaciones y Control. }\end{array}$ \\
\hline & Sistema Procesador \\
de Cronogramas & $\begin{array}{l}\text { Se encarga de procesar los cronogramas e identificar } \\
\text { si existen variaciones con lo planificado, de ser así, } \\
\text { se generará una Solicitud de cambio ya sea por los } \\
\text { motivos de: esfuerzo, duración o hitos. }\end{array}$ \\
\hline
\end{tabular}

Nota: Se ha descrito las aplicaciones existentes del proceso de gestión de proyectos. Adaptado de "Manual de Configuración”, por El Objeto de Estudio, 2017. 


\section{(1)}

4. ARQUITECTURA TECNOLÓGICA

○ COMPONENTES DE TECNOLOGÍA Y SUS RELACIONES CON LOS SISTEMAS INFORMACIÓN

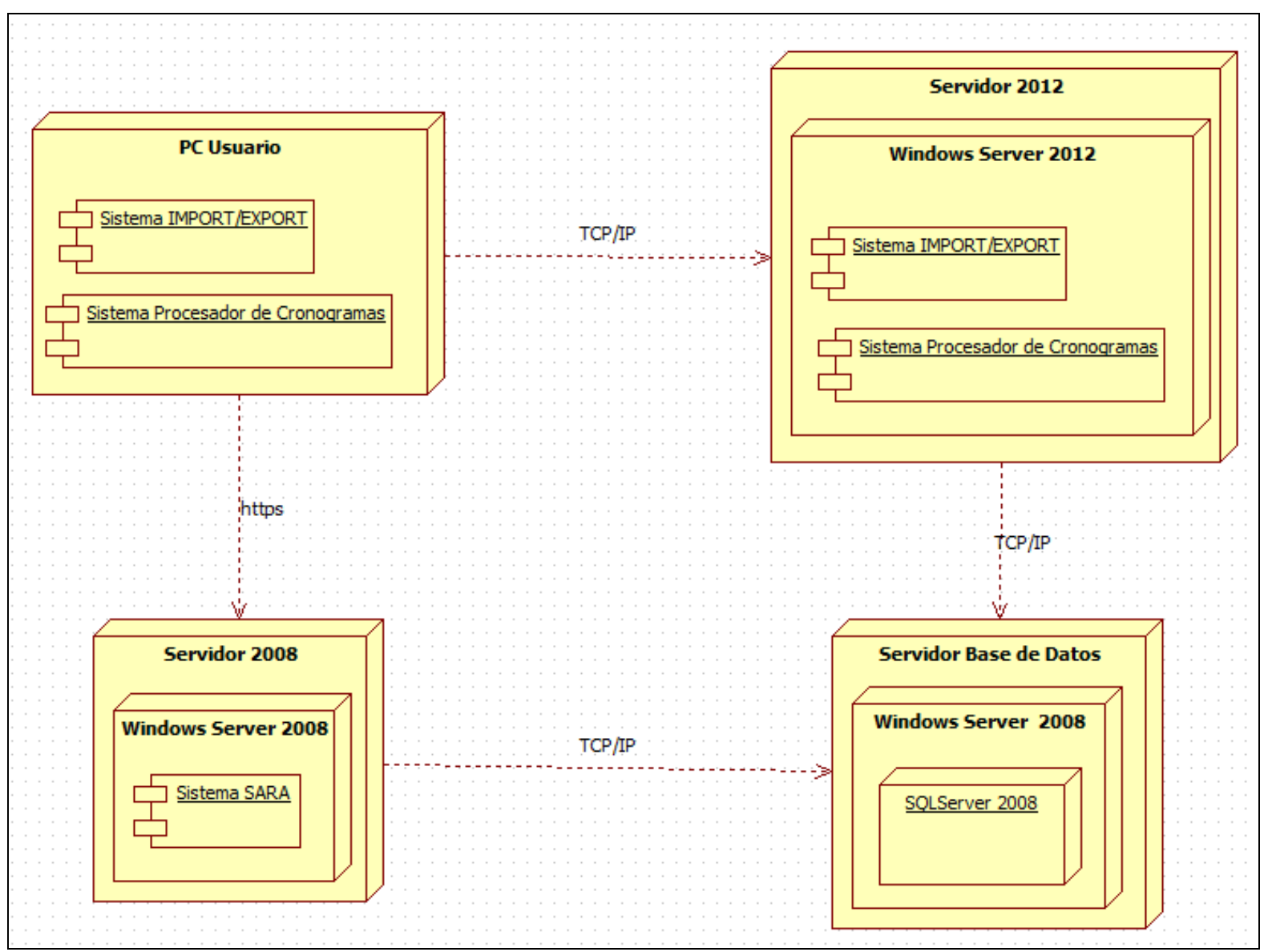

Figura 17. Diagrama de Componentes del Proceso de Gestión de Proyectos (ASIS). Adaptado de "Manual de Configuración”, por El Objeto de Estudio, 2017.

\section{○ PLATAFORMA DE TECNOLOGÍA Y SU DESCOMPOSICIÓN}

Tabla 45

Detalle de Componentes del Proceso Gestión de Proyectos (ASIS)

\begin{tabular}{|l|l|}
\hline \multicolumn{2}{|l|}{ IMPORT/EXPORT } \\
\hline Servidor & Servidor 2012 \\
\hline Sistema Operativo & Windows Server 2012 \\
\hline Disco Duro & 5 Terabytes \\
\hline SISTEMA SARA & \\
\hline Servidor & Servidor 2008 \\
\hline
\end{tabular}




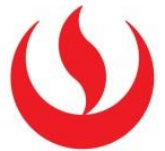

\begin{tabular}{|l|l|}
\hline Sistema Operativo & Windows Server 2008 \\
\hline Disco Duro & 5 Terabytes \\
\hline PROCESADOR CRONOGRAMAS \\
\hline Servidor & Servidor 2012 \\
\hline Sistema Operativo & Windows Server 2012 \\
\hline Disco Duro & 5 Terabytes \\
\hline SQLServer 2008 & \\
\hline Servidor & Servidor Base Datos \\
\hline Sistema Operativo & Windows Server 2008 \\
\hline Disco Duro & 5 Terabytes \\
\hline
\end{tabular}

Nota: Se ha descrito el servidor, sistema operativo y disco duro por cada aplicación. Adaptado de "Manual de Configuración”, por El Objeto de Estudio, 2017.

\section{○ AMBIENTES Y UBICACIONES}

Tabla 46

Detalle de Ubicaciones y Ambientes (ASIS)

\begin{tabular}{|c|c|c|c|}
\hline UBICACIÓN & AMBIENTES & SERVIDORES & DETALLE \\
\hline \multirow[t]{4}{*}{ PISO 1} & Recepción & & \\
\hline & Directorio & & \\
\hline & $\begin{array}{c}\text { Gerencia de Investigación } \\
\text { y Desarrollo }\end{array}$ & & \\
\hline & Desarrollo V & Servidor EFT & $\begin{array}{l}\text { Tipo: Físico } \\
\text { Procesador: Supermicro } \\
\text { Aplicaciones: Oracle Linux } \\
\text { Discos:3 } \\
\text { Capacidad: 160G, 2T, } 2 \mathrm{~T} \\
\text { Potencia 350W }\end{array}$ \\
\hline \multirow[t]{3}{*}{ PISO 2} & Gerencia de Soporte & Servidor RSA & $\begin{array}{l}\text { Tipo: Físico } \\
\text { Procesador: Intel } \\
\text { Aplicaciones: AIX P520 } \\
\text { Discos:1 } \\
\text { Capacidad: 1T } \\
\text { Potencia 350W }\end{array}$ \\
\hline & & Servidor Soporte & $\begin{array}{l}\text { Tipo: Físico } \\
\text { Procesador: Intel } \\
\text { Aplicaciones: Oracle Linux } \\
\text { Discos:2 } \\
\text { Capacidad: } 500 \mathrm{G}, 1 \mathrm{~T} \\
\text { Potencia 350W }\end{array}$ \\
\hline & Control & Servidor Certificación & $\begin{array}{l}\text { Tipo: Físico } \\
\text { Procesador: Supermicro }\end{array}$ \\
\hline
\end{tabular}




\begin{tabular}{|c|c|c|c|}
\hline UBICACIÓN & AMBIENTES & SERVIDORES & DETALLE \\
\hline & & & $\begin{array}{l}\text { Aplicaciones: Oracle Linux } \\
\text { Discos:3 } \\
\text { Capacidad: 1T, 1T, 1T } \\
\text { Potencia 350W }\end{array}$ \\
\hline & & Servidor de Control & $\begin{array}{l}\text { Tipo: Físico } \\
\text { Procesador: Intel } \\
\text { Aplicaciones: Server } 2012 \\
\text { Discos:2 } \\
\text { Capacidad: 2T, 2T } \\
\text { Potencia 350W }\end{array}$ \\
\hline & Gerencia de Marketing & Servidor Marketing & $\begin{array}{l}\text { Tipo: Físico } \\
\text { Procesador: Intel } \\
\text { Aplicaciones: Server2008 } \\
\text { Discos:1 } \\
\text { Capacidad: 2T } \\
\text { Potencia 350W }\end{array}$ \\
\hline & Gerencia de CAT 01 & Servidor CAT 1 & $\begin{array}{l}\text { Tipo: Físico } \\
\text { Procesador: Supermicro } \\
\text { Aplicaciones: Oracle Linux } \\
\text { Discos:3 } \\
\text { Capacidad: } 1 \mathrm{~T}, 2 \mathrm{~T}, 2 \mathrm{~T} \\
\text { Potencia 350W }\end{array}$ \\
\hline & Gerencia de CAT 02 & Servidor SPA & $\begin{array}{l}\text { Tipo: Físico } \\
\text { Procesador: Supermicro } \\
\text { Aplicaciones: Oracle Linux } \\
\text { Discos:2 } \\
\text { Capacidad: } 1 \mathrm{~T}, 1 \mathrm{~T} \\
\text { Potencia } 350 \mathrm{~W}\end{array}$ \\
\hline \multirow[t]{4}{*}{ PISO 3} & \multirow[t]{4}{*}{ Sala de servidores } & Servidor Antispam & $\begin{array}{l}\text { Tipo: Físico } \\
\text { Procesador: Intel } \\
\text { Aplicaciones: Linux } 6.5 \\
\text { Discos:1 } \\
\text { Capacidad: } 160 \mathrm{G} \\
\text { Potencia 350W }\end{array}$ \\
\hline & & Servidor Exchange & $\begin{array}{l}\text { Tipo: Físico } \\
\text { Procesador: Intel } \\
\text { Aplicaciones: Windows Server } 2003 \\
\text { Discos:1 } \\
\text { Capacidad: 1T } \\
\text { Potencia 350W }\end{array}$ \\
\hline & & Servidor Firewall & $\begin{array}{l}\text { Tipo: Físico } \\
\text { Procesador: Intel } \\
\text { Aplicaciones: Linux } 6.5 \\
\text { Discos:1 } \\
\text { Capacidad: } 1 \mathrm{~T} \\
\text { Potencia 350W }\end{array}$ \\
\hline & & Servidor 2008 & Tipo: Físico \\
\hline
\end{tabular}




\begin{tabular}{|c|c|c|c|}
\hline UBICACIÓN & AMBIENTES & SERVIDORES & DETALLE \\
\hline & & & $\begin{array}{l}\text { Procesador: Intel } \\
\text { Aplicaciones: Server } 2008 \\
\text { Discos:1 } \\
\text { Capacidad: 1T } \\
\text { Potencia 350W }\end{array}$ \\
\hline & & Servidor IBM P720 & $\begin{array}{l}\text { Tipo: Físico } \\
\text { Procesador: IBM } \\
\text { Aplicaciones: AIX } 720 \\
\text { Discos:1 } \\
\text { Capacidad: 600G } \\
\text { Potencia 350W }\end{array}$ \\
\hline & & Servidor 2012 & $\begin{array}{l}\text { Tipo: Físico } \\
\text { Procesador: Intel } \\
\text { Aplicaciones: Server } 2012 \\
\text { Discos:1 } \\
\text { Capacidad: } 2 \mathrm{~T} \\
\text { Potencia 350W }\end{array}$ \\
\hline & & Servidor IBM P520 & $\begin{array}{l}\text { Tipo: Físico } \\
\text { Procesador: IBM } \\
\text { Aplicaciones: AIX } 520 \\
\text { Discos:1 } \\
\text { Capacidad: 600G } \\
\text { Potencia 350W }\end{array}$ \\
\hline & & Servidor OV Manager & $\begin{array}{l}\text { Tipo: Físico } \\
\text { Procesador: IBM } \\
\text { Aplicaciones: Oracle Linux } 5 \\
\text { Discos:1 } \\
\text { Capacidad: 1T } \\
\text { Potencia 350W }\end{array}$ \\
\hline & & Servidor Sharepoint & $\begin{array}{l}\text { Tipo: Físico } \\
\text { Procesador: Intel } \\
\text { Aplicaciones: Server } 2016 \\
\text { Discos:1 } \\
\text { Capacidad: } 2 \mathrm{~T} \\
\text { Potencia 350W }\end{array}$ \\
\hline & & Servidor Asterisk & $\begin{array}{l}\text { Tipo: Físico } \\
\text { Procesador: IBM } \\
\text { Aplicaciones: Linux } 5 \\
\text { Discos:1 } \\
\text { Capacidad: 75G } \\
\text { Potencia 350W }\end{array}$ \\
\hline & & $\begin{array}{l}\text { Servidor de Base de } \\
\text { Datos 10gr2 }\end{array}$ & $\begin{array}{l}\text { Tipo: Físico } \\
\text { Procesador: Intel } \\
\text { Aplicaciones: Linux } \\
\text { Discos:2 } \\
\text { Capacidad: 250G, 320G } \\
\text { Potencia 350W }\end{array}$ \\
\hline
\end{tabular}




\section{(4)}

\begin{tabular}{|c|c|c|c|}
\hline UBICACIÓN & AMBIENTES & SERVIDORES & DETALLE \\
\hline & & Servidor de Cámaras & $\begin{array}{l}\text { Tipo: Físico } \\
\text { Procesador: Intel } \\
\text { Aplicaciones: Windows } 7 \\
\text { Discos:1 } \\
\text { Capacidad: 1T } \\
\text { Potencia 350W }\end{array}$ \\
\hline & & Servidor Producción & $\begin{array}{l}\text { Tipo: Físico } \\
\text { Procesador: Supermicro } \\
\text { Aplicaciones: Oracle, Linux } \\
\text { Discos:3 } \\
\text { Capacidad: 500G, 1T, 1T } \\
\text { Potencia 350W }\end{array}$ \\
\hline & & Servidor Subversion & $\begin{array}{l}\text { Tipo: Físico } \\
\text { Procesador: Intel } \\
\text { Aplicaciones: Linux } \\
\text { Discos:1 } \\
\text { Capacidad: 1T } \\
\text { Potencia 350W }\end{array}$ \\
\hline & & Servidor Shared & $\begin{array}{l}\text { Tipo: Físico } \\
\text { Procesador: Supermicro } \\
\text { Aplicaciones: Oracle, Linux } \\
\text { Discos:3 } \\
\text { Capacidad: 1T, 2T, 2T } \\
\text { Potencia 350W }\end{array}$ \\
\hline & & $\begin{array}{l}\text { Servidor de Base de } \\
\text { Datos } 11 \mathrm{gr} 2\end{array}$ & $\begin{array}{l}\text { Tipo: Físico } \\
\text { Procesador: Intel } \\
\text { Aplicaciones: Linux } \\
\text { Discos:1 } \\
\text { Capacidad: 500G } \\
\text { Potencia 350W }\end{array}$ \\
\hline & & $\begin{array}{l}\text { Servidor de Base de } \\
\text { Datos } 11 \mathrm{gr} 3\end{array}$ & $\begin{array}{l}\text { Tipo: Físico } \\
\text { Procesador: Intel } \\
\text { Aplicaciones: Linux } \\
\text { Discos:1 } \\
\text { Capacidad: 1T } \\
\text { Potencia 350W }\end{array}$ \\
\hline & & Servidor Vware & $\begin{array}{l}\text { Tipo: Físico } \\
\text { Procesador: Intel } \\
\text { Aplicaciones: Linux } 5.5 \\
\text { Discos:1 } \\
\text { Capacidad: 2T } \\
\text { Potencia 350W }\end{array}$ \\
\hline & Administración & & \\
\hline
\end{tabular}

Nota: Se ha descrito el tipo, procesador, aplicación discos, capacidad y potencia de cada servidor. Adaptado de "Manual de Configuración", por El Objeto de Estudio, 2017. 


\section{(1)}

○ COMUNICACIONES FÍSICAS / ESPECIFICACIONES DE HARDWARE Y RED

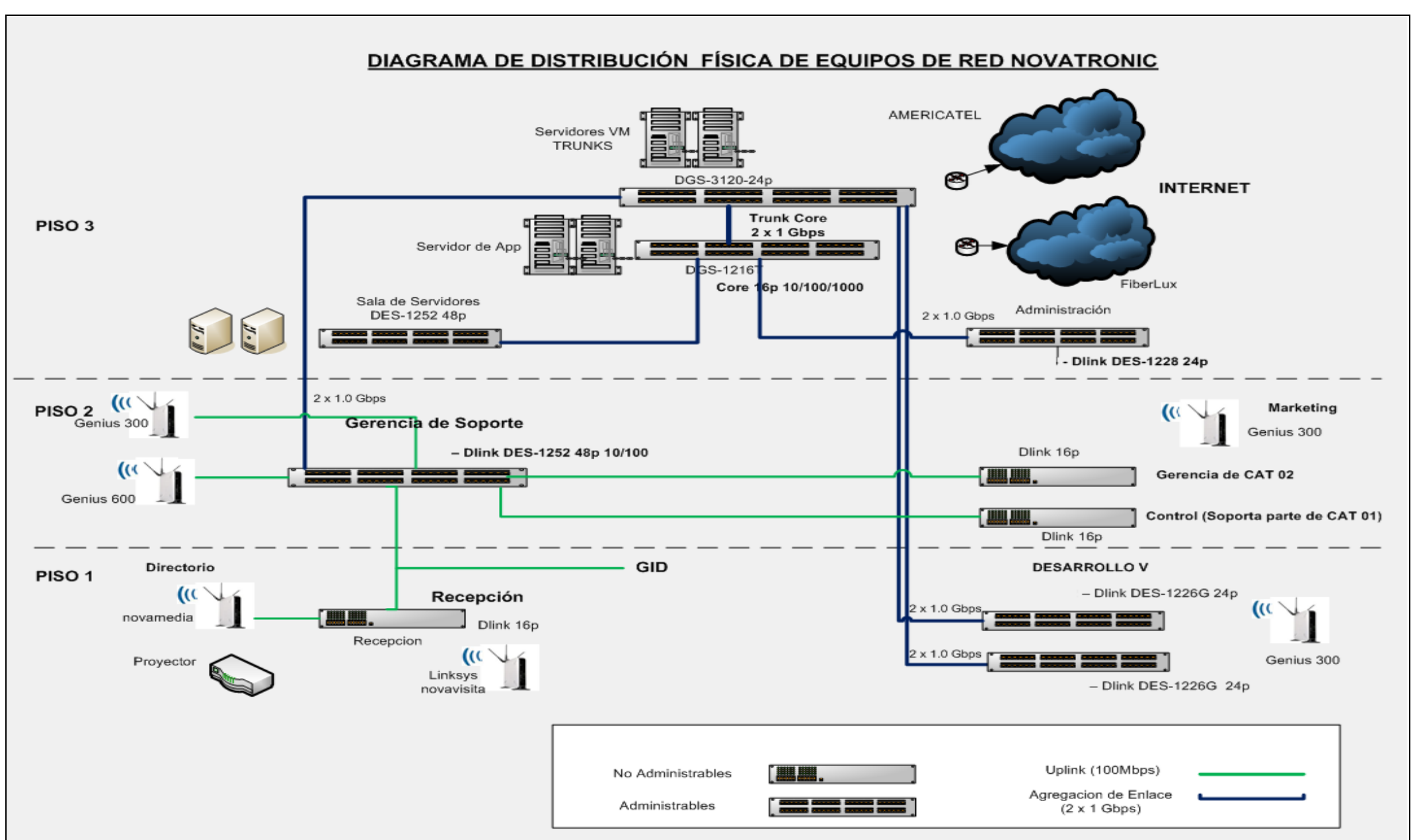

Figura 18. Diagrama de Distribución Física de Equipos de Red (ASIS). Adaptado de “Manual de Configuración”, por El Objeto de Estudio, 2017. 


\section{(1)}

PISO $1 \rightarrow$ Áreas: Recepción, Directorio, Gerencia de Investigación y Desarrollo y Desarrollo V.

Tabla 47

Componentes del Piso 1 (ASIS)

\begin{tabular}{|l|l|}
\hline Componente & Descripción \\
\hline 6 Switch Dlink & Switchs de marca Dlink con potencia de 100 watts cada uno. \\
\hline 1 Proyector & Marca EPSON. \\
\hline
\end{tabular}

Nota: Se ha descrito los componentes del piso1. Adaptado de "Manual de Configuración", por El Objeto de Estudio, 2017.

PISO $2 \rightarrow$ Áreas: Gerencia de Soporte, de Marketing, de CAT 01, de CAT 02 y Control Tabla 48

Componentes del Piso 2 (ASIS)

\begin{tabular}{|l|l|}
\hline Componente & Descripción \\
\hline 6 Switch Dlink & Switchs de marca Dlink con potencia de 100 watts cada uno. \\
\hline
\end{tabular}

Nota: Se ha descrito los componentes del piso2. Adaptado de "Manual de Configuración", por El Objeto de Estudio, 2017.

PISO $3 \rightarrow$ Áreas: Sala de servidores y Administración

Tabla 49

Componentes del Piso 3 (ASIS)

\begin{tabular}{|c|c|}
\hline Componente & Descripción \\
\hline Servidores & Sala de servidores donde se alojan la gran parte de equipos \\
\hline $\begin{array}{l}\text { Servidor } \\
\text { TRUNKS }\end{array}$ & $\begin{array}{l}\text { Servidor con sistema operativo Linux } 5.5 \text { y procesador Intel. Capacidad de } \\
2 \text { terabytes distribuido en } 2 \text { discos de almacenamiento y con potencia de } \\
350 \text { watts. }\end{array}$ \\
\hline Servidor de App & $\begin{array}{l}\text { Servidor con sistema operativo Linux } 5.5 \text { y procesador Intel. Capacidad de } \\
2 \text { terabytes distribuido en } 2 \text { discos de almacenamiento y con potencia de } \\
350 \text { watts. }\end{array}$ \\
\hline 2 Switch Dlink & Switchs de marca Dlink con potencia de 100 watts cada uno. \\
\hline Router Fiberlux & Router de marca Zycel con potencia de 100 watts. \\
\hline Router Americatel & Router de marca Cisco modelo 2901 con potencia de 400 watts. \\
\hline
\end{tabular}

Nota: Se ha descrito los componentes del piso 3. Adaptado de "Manual de Configuración", por El Objeto de Estudio, 2017. 


\section{(1)}

\section{FUNDAMENTO Y JUSTIFICACIÓN DEL ENFOQUE ARQUITECTONICO}

Según lo detallado en la arquitectura empresarial AS IS y el proceso de negocio analizado, se precisan los principales problemas y/o requerimientos a resolver en la propuesta de arquitectura empresarial TO BE.

\section{Problemática del proceso}

- El control manual del cronograma del proyecto no es óptimo.

- Mala gestión de los entregables del proyecto.

- No existe algún responsable para el seguimiento de la creación / actualización de los entregables

\section{Principales requerimientos}

- Contar con un sistema, que sustituya la creación / actualización manual del cronograma, que permita llevar un seguimiento en tiempo real de las actividades del proyecto.

- Contar con un sistema, que sustituya la creación / actualización de la matriz de trazabilidad, que permita realizar el cruce de los entregables con los requerimientos.

- Contar con un sistema, que sustituya la creación / actualización de los Riesgos del proyecto, que permita llevar un seguimiento de los riesgos del proyecto.

- Contar con un sistema, que sustituya la creación / actualización de la Bitácora del proyecto, que permita llevar un seguimiento del proyecto.

- Implementar nuevos equipos y licencias que den soporte a la solución propuesta.

- Actualización del procedimiento de Gestión de Proyectos, que permita establecer un punto de control para el seguimiento de los entregables y contenga la referencia de la nueva herramienta de software. 


\section{ARQUITECTURA DE NEGOCIO DE DESTINO}

1. ARQUITECTURA DE NEGOCIO

- ESTRUCTURA DE LA ORGANIZACIÓN

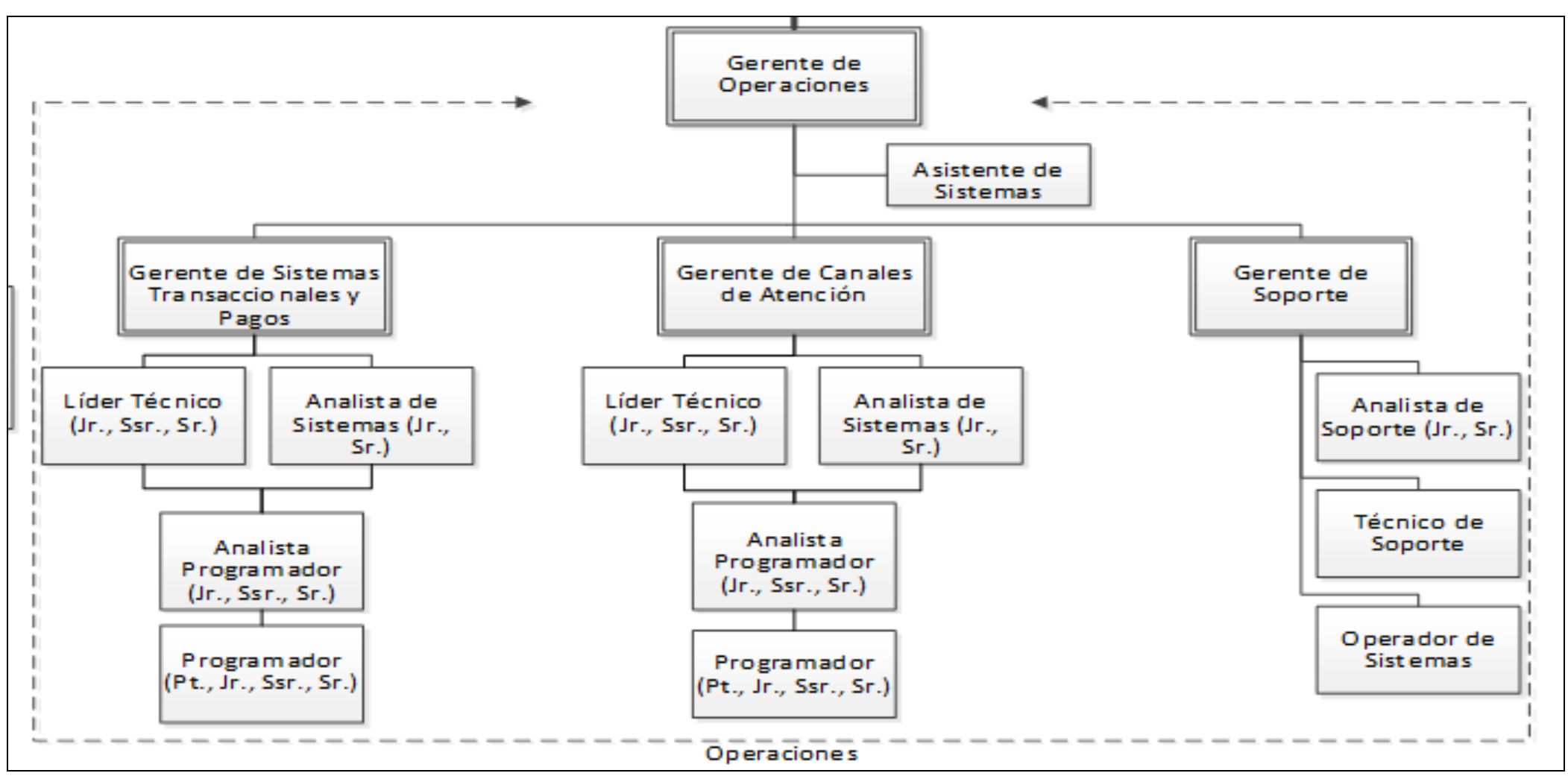

Figura 19. Organigrama de la empresa - Proceso Seleccionado (TOBE). Adaptado de "Manual de funciones y perfiles de puestos", por El Objeto de Estudio, 2017. 


\section{(1)}

Tal como indica el Objeto de Estudio (2017), a continuación se describen brevemente los roles indicados en el organigrama:

\section{Gerencia de Operaciones}

1.1. Gerente de Operaciones: Encargado de planificar, organizar, dirigir, y controlar las actividades y procedimientos de su Gerencia, con la finalidad de asegurar la administración eficiente de los recursos humanos, financieros, materiales y servicios requeridos para el correcto desarrollo de los proyectos y productos, así como brindar un óptimo mantenimiento y asistencia técnica a los clientes; contribuyendo así al cumplimiento de la misión y objetivos institucionales.

1.2. Asistente de Sistemas: Encargado de colaborar con las tareas administrativas y de gestión de las Gerencias de Producto, a fin de contribuir con el cumplimiento de los cronogramas y objetivos trazados para dichas áreas.

1.3. Gerente de Canales de Atención: Encargado de planificar, organizar, dirigir y controlar las actividades del área de Canales de Atención, con el objetivo de lograr la satisfacción de los clientes mediante una adecuada gestión de los proyectos y productos asignados así como del cumplimiento del Sistema de Gestión de la Calidad (SGC).

1.4. Gerente de Sistemas Transaccionales y Pagos: Encargado de planificar, organizar, dirigir y controlar las actividades del área de Sistemas de Pago, con el objetivo de lograr la satisfacción de los clientes mediante una adecuada gestión de los proyectos y productos asignados así como del cumplimiento del Sistema de Gestión de la Calidad (SGC).

1.5. Líder Técnico: Encargado de velar por el cumplimiento técnico de los productos, sistemas y servicios de la empresa, considerando los intereses / requerimientos técnicos tales como arquitectura, buenas prácticas y estándares de la empresa y de sus clientes. 
1.6. Analista de Sistemas: Encargado de analizar, diseñar, desarrollar e implementar productos y/o adecuaciones de los mismos cumpliendo las normas y procedimientos establecidos, a fin de satisfacer los requerimientos de los clientes e internos, garantizando la calidad del producto y la satisfacción del cliente.

1.7. Analista Programador: Encargado de analizar y describir los requerimientos para elaborar especificaciones funcionales y técnicas, así como ejecutar las actividades para convertir dichas especificaciones en un código de software, a fin de garantizar la calidad del producto y la satisfacción del cliente.

1.8. Programador: Encargado de ejecutar las tareas de programación e implementación de los proyectos y atenciones de requerimiento asignados aplicando los estándares de programación, las normas y procedimientos de la Institución para entregar un producto de calidad que cumpla con los requerimientos del cliente.

1.9. Gerente de Soporte: Encargado de brindar solución a los problemas que reporten los clientes y/o usuarios del Objeto de Estudio, de la manera más eficiente, en los tiempos acordados, proveyendo un soporte especializado y ágil con conocimiento y herramientas de TI, con el objetivo de asegurar un nivel de satisfacción elevado en los mismos.

1.10. Analista de Soporte: Encargado de realizar el análisis y corrección de los reportes de problemas reportados por los clientes, así como atender sus consultas en las implementaciones de producción, a fin de contribuir a que funcionen correctamente según lo definido en la documentación correspondiente.

1.11. Técnico de Soporte: Encargado de velar porque la infraestructura tecnológica de la empresa se encuentre operativa con el fin de permitir el desarrollo de las operaciones de la empresa.

1.12. Operador de Sistemas: Encargado de mantener la operatividad y continuidad del servicio "Sistema de procesamiento transaccional", a fin de garantizar en todo momento la disponibilidad del servicio frente a los clientes y autorizadores, en base al manejo de procedimientos y herramientas de gestión propias del sistema. 


\section{(1)}

○ MAPA DE PROCESOS Y FUNCIONES DE NEGOCIO

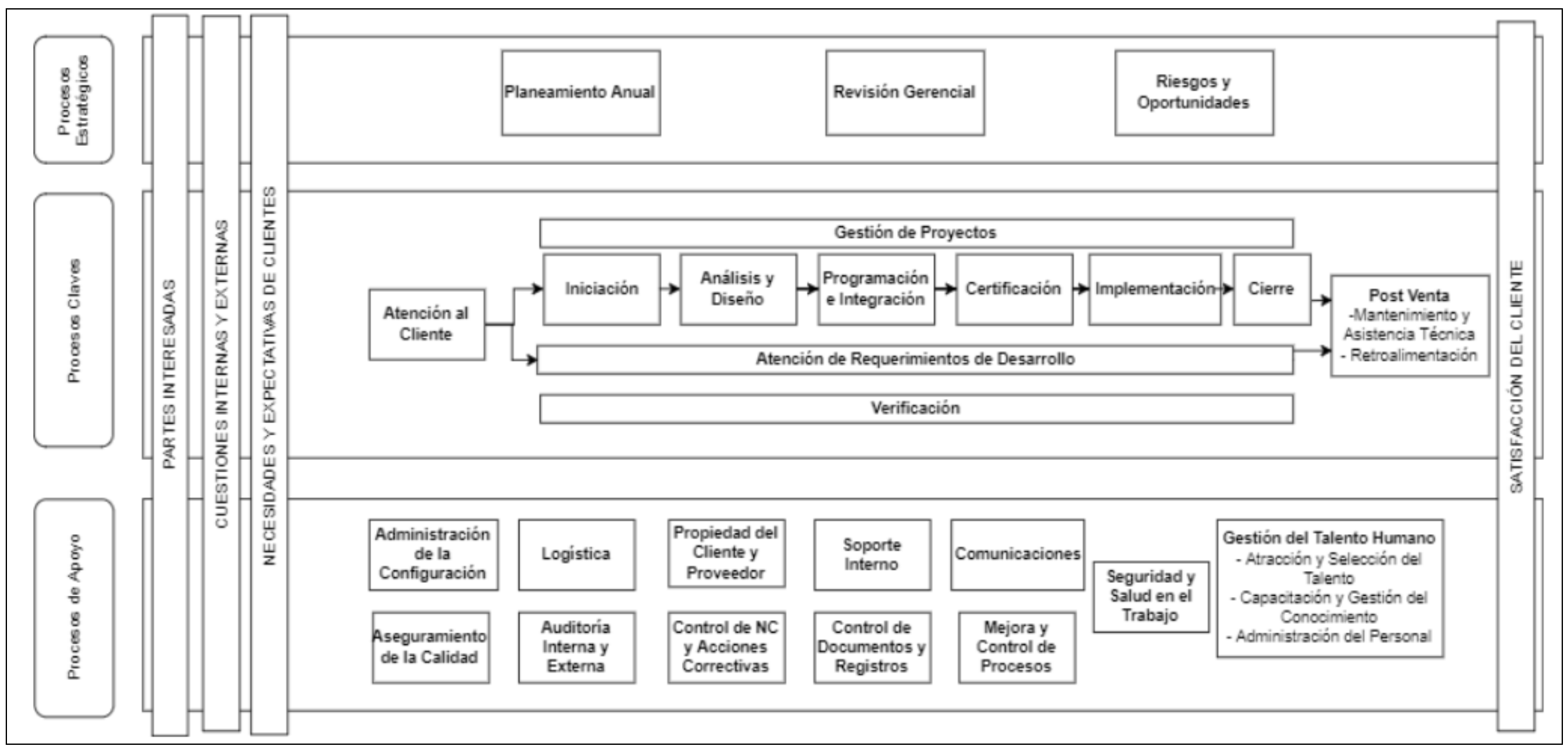

Figura 20. Mapa de procesos de la empresa (TOBE). Adaptado de "Manual de calidad”, por El Objeto de Estudio, 2017. 


\section{$(1$}

Tabla 50

Objetivos de los Procesos de Negocios (TOBE)

\begin{tabular}{|c|c|c|}
\hline $\mathbf{N}^{\mathbf{o}}$ & Procesos Claves & OBJETIVO DEL PROCESO \\
\hline 1 & Atención al Cliente & $\begin{array}{l}\text { Mantener una retroalimentación continua de las necesidades de los clientes: En } \\
\text { este proceso se elaboran las encuestas de satisfacción global y de proyectos, la } \\
\text { canalización de quejas y la atención de propuestas comerciales. } \\
\text { Recolectar requerimientos y necesidades de los clientes y elaborar propuestas } \\
\text { comerciales que le den frente. }\end{array}$ \\
\hline 2 & $\begin{array}{l}\text { Gestión } \\
\text { Proyectos }\end{array}$ & 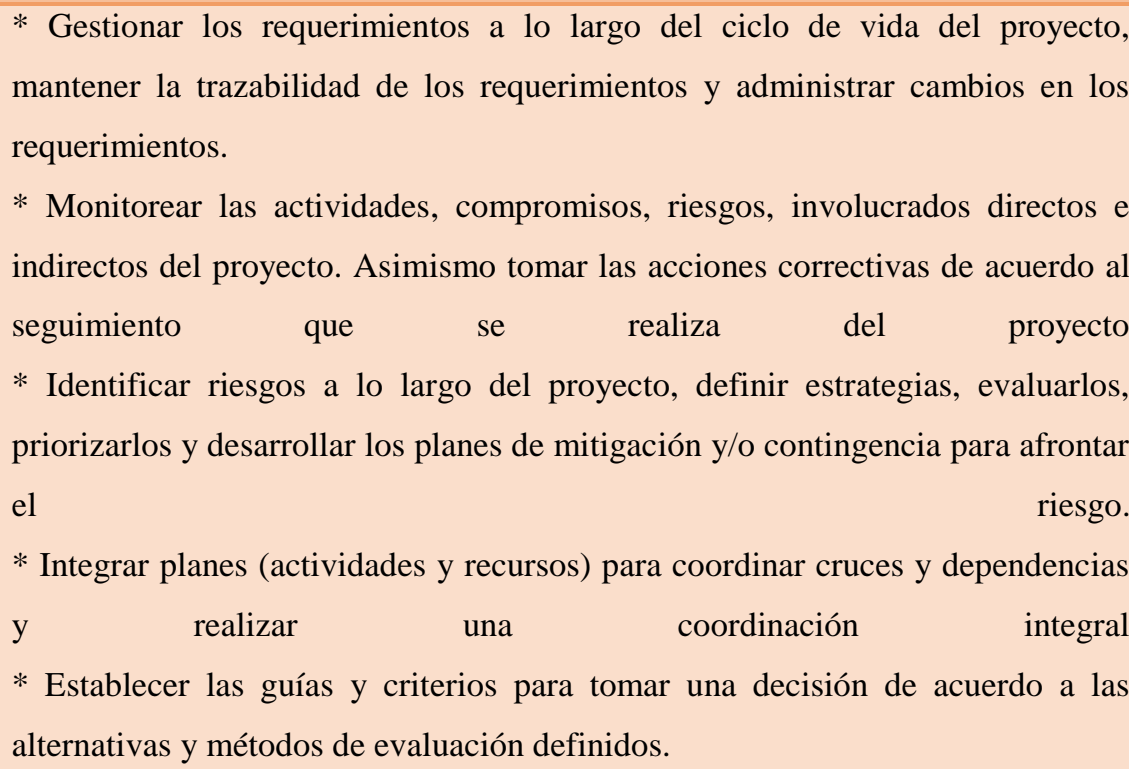 \\
\hline 3 & Iniciación & 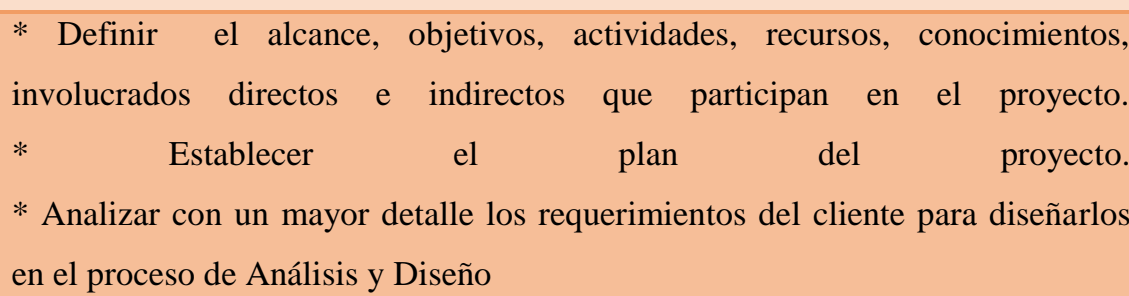 \\
\hline 4 & Análisis y Diseño & $\begin{array}{l}\text { * Elaborar el diseño en función de los requerimientos detallados en Iniciación } \\
\text { para establecer el esquema operacional, la funcionalidad requerida, las } \\
\text { interfaces } \\
\text { * necesarias, } \\
\text { etc. } \\
\text { funcionalidad requerida, en base a las cuales se tome una decisión, diseñar el } \\
\text { producto y sus componentes, diseñar las interfaces, hacer, utilizar, reusar o } \\
\text { comprar los componentes para el producto. }\end{array}$ \\
\hline 5 & $\begin{array}{l}\text { Programación } \\
\text { Integración }\end{array}$ & $\begin{array}{l}\text { * Implementar el diseño, elaborar la documentación necesaria, construcción e } \\
\text { integrar los diferentes componentes del producto, además de validar esta } \\
\text { integración. } \\
\text { * Realizar la preparación y ejecución de las pruebas del producto elaborado }\end{array}$ \\
\hline
\end{tabular}




\section{()}

\begin{tabular}{|c|c|c|}
\hline 6 & Certificación & $\begin{array}{l}\text { Realizar la validación del producto mediante la ejecución de pruebas de } \\
\text { certificación. }\end{array}$ \\
\hline 7 & Implementación & $\begin{array}{l}\text { Instalar el producto desarrollado en los ambientes del cliente para la ejecución } \\
\text { de pruebas finales. }\end{array}$ \\
\hline 8 & Cierre & $\begin{array}{l}\text { Documentar la evaluaciones y otros requisitos para gestionar el cierre del } \\
\text { proyecto en el sistema SARA }\end{array}$ \\
\hline 9 & Verificación & $\begin{array}{l}\text { Realizar la verificación de los entregables del proceso productivo de acuerdo a } \\
\text { criterios establecidos previamente. }\end{array}$ \\
\hline 10 & $\begin{array}{l}\text { Mantenimiento y } \\
\text { Asistencia Técnica }\end{array}$ & $\begin{array}{l}\text { Atender la cartera de clientes con necesidades de mantenimiento y asistencia } \\
\text { técnica, incluye la atención de reportes de problemas y mantenimientos que no } \\
\text { impliquen una modificación o ajuste en el producto. }\end{array}$ \\
\hline 11 & $\begin{array}{l}\text { Atención de } \\
\text { Requerimientos de } \\
\text { Desarrollo, Ajustes } \\
\text { y Modificaciones }\end{array}$ & $\begin{array}{l}\text { Gestionar la cartera de desarrollos, ajustes y modificaciones. Incluye la atención } \\
\text { de Reportes de problemas que implican un ajuste o modificación en el producto. }\end{array}$ \\
\hline $\mathbf{N}^{\mathbf{0}}$ & $\begin{array}{l}\text { Procesos } \\
\text { Estratégicos }\end{array}$ & OBJETIVO DEL PROCESO \\
\hline 12 & Planeamiento Anual & Realizar el plan anual de inversiones de la empresa. \\
\hline 13 & Revisión Gerencial & $\begin{array}{l}\text { Realizar seguimiento a los acuerdos plasmados en las reuniones de revisión } \\
\text { gerencial anuales. }\end{array}$ \\
\hline 14 & $\begin{array}{l}\text { Gestión de Riesgos } \\
\text { y Oportunidades }\end{array}$ & Gestionar los riesgos y oportunidades, que son de aplicación a la organización. \\
\hline $\mathbf{N}^{\mathbf{0}}$ & Proceso de Apoyo & OBJETIVO DEL PROCESO \\
\hline 15 & Logística & $\begin{array}{l}\text { Gestionar los recursos necesarios para todas las áreas de la empresa poniendo } \\
\text { especial énfasis en los recursos que impactan directamente en la calidad del } \\
\text { producto (servicios externos y otros proveedores) }\end{array}$ \\
\hline 16 & $\begin{array}{l}\text { Gestión de Talento } \\
\text { Humano }\end{array}$ & $\begin{array}{l}\text { Gestionar la capacitación interna y la contratación de personal idóneo para el } \\
\text { desempeño de los procesos. }\end{array}$ \\
\hline 17 & Soporte Interno & Brindar soporte interno a los usuarios de la empresa. \\
\hline 18 & $\begin{array}{l}\text { Administración de } \\
\text { la Configuración }\end{array}$ & $\begin{array}{l}\text { Mantener el orden, control de cambios y trazabilidad de los productos de la } \\
\text { empresa }\end{array}$ \\
\hline 19 & $\begin{array}{l}\text { Propiedad del } \\
\text { Cliente }\end{array}$ & Registrar y administrar la propiedad del cliente utilizada en los proyectos. \\
\hline 20 & $\begin{array}{l}\text { Aseguramiento de } \\
\text { la Calidad }\end{array}$ & Verificación documental de la línea base establecidas para los proyectos \\
\hline 21 & Mejora y Control de & Dar los lineamientos para la gestión de nuevos procesos en la empresa \\
\hline
\end{tabular}




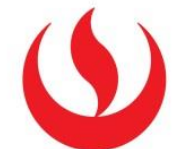

\begin{tabular}{|l|lr|l|}
\hline 22 & $\begin{array}{l}\text { Procesos } \\
\begin{array}{l}\text { Control de } \\
\text { Conformidades }\end{array} \\
\text { Acciones } \\
\text { Correctivas }\end{array}$ & $\begin{array}{l}\text { Gestionar las no conformidades, potenciales no conformidades, así como las } \\
\text { acciones correctivas que ameriten. }\end{array}$ \\
\hline 23 & $\begin{array}{l}\text { Auditoría Interna y } \\
\text { Externa }\end{array}$ & $\begin{array}{l}\text { Gestionar las auditorías internas y externas de la empresa asi como las } \\
\text { observaciones o no conformidades originadas en las mismas. }\end{array}$ \\
\hline $\mathbf{2 4}$ & $\begin{array}{l}\text { Control } \\
\text { Documentos } \\
\text { Registros }\end{array}$ & ye & $\begin{array}{l}\text { Establecer las pautas para la elaboración, modificación, publicación y control de } \\
\text { documentos y registros. }\end{array}$ \\
\hline $\mathbf{2 5}$ & $\begin{array}{l}\text { Comunicaciones } \\
\text { Gestión de la comunicación efectiva en la empresa, tanto de manera interna, } \\
\text { como hacia las partes interesadas. En este proceso se definirán los canales de } \\
\text { comunicación internos y externos establecidos por la empresa. } \\
\text { Mantener al personal interno/externo informado acerca de los asuntos y } \\
\text { decisiones relativas al funcionamiento del Sistema de Gestión de la Calidad. }\end{array}$ \\
\hline $\mathbf{2 6}$ & $\begin{array}{l}\text { Seguridad y } \\
\text { en el Trabajo }\end{array}$ & $\begin{array}{l}\text { Mantener el Sistema de Gestión de Seguridad y Salud en el Trabajo en la } \\
\text { organización y cumplir con la normatividad nacional vigente aplicable en } \\
\text { materia de riesgos laborales. }\end{array}$ \\
\hline
\end{tabular}

Nota: Se ha descrito los objetivos de los procesos claves, estratégicos y de apoyo. Adaptado de "Manual de calidad”, por El Objeto de Estudio, 2017. 


\section{○ MATRIZ DE OBJETIVOS DEL NEGOCIO VS PROCESOS}

Los objetivos estratégicos son los objetivos planteados por una organización para así lograr determinadas metas. Al respecto, el Objeto de

Estudio (2017) señala que:

1. Objetivo Estratégico 1: OE1: Contar con un índice de Satisfacción Global por Cliente en un 82\%. (Servicio integral: Soporte, Actividades, Proyectos).

2. Objetivo Estratégico 2: OE2: Lograr que el 90\% de personal del área de Operaciones tenga como mínimo 25 horas de capacitación orientadas al logro del incremento de la productividad.

3. Objetivo Estratégico 3: OE3: Crear por lo menos 2 nuevos productos competitivos durante el año, y actualizar 2 de los actuales.

4. Objetivo Estratégico 4: OE4: Invertir el 8\% de las ventas en la investigación y desarrollo tecnológico orientado a la productividad y competitividad.

5. Objetivo Estratégico 5: OE5: Incrementar en un 10\% la productividad mediante el mejoramiento de nuestros procesos (menos errores y velocidad de ejecución). (p. 16). 


\section{(}

Tabla 51

Matriz de Objetivos Estratégicos vs Procesos (TOBE)

\begin{tabular}{|c|c|c|c|c|c|c|c|c|c|c|c|c|c|c|c|c|c|c|c|c|c|c|c|c|c|c|c|}
\hline \multirow{3}{*}{ Estratégicos } & \multicolumn{27}{|c|}{ PROCESOS } \\
\hline & \multicolumn{3}{|c|}{ Estratégicos } & \multicolumn{13}{|c|}{ Apoyo } & \multicolumn{11}{|c|}{ Claves } \\
\hline & 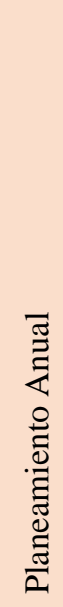 & 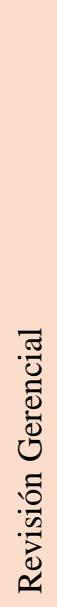 & 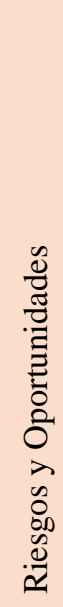 & 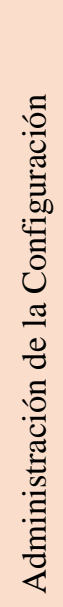 & 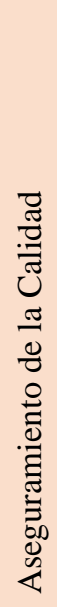 & 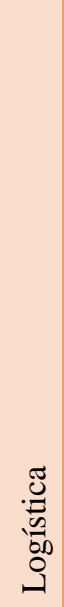 & 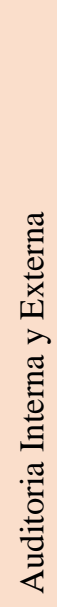 & 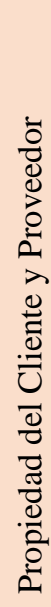 & 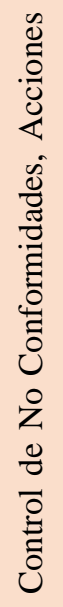 & 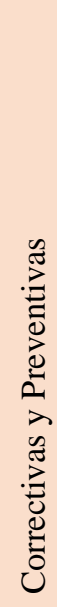 & 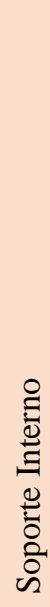 & 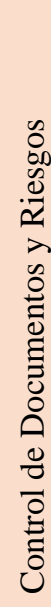 & 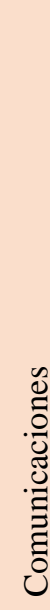 & 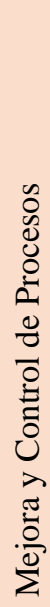 & 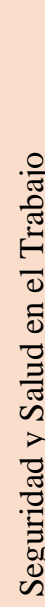 & 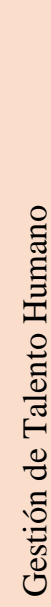 & 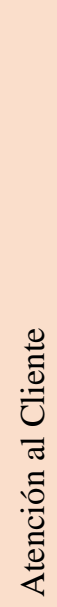 & 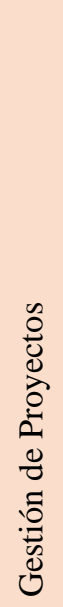 & 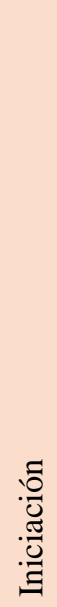 & 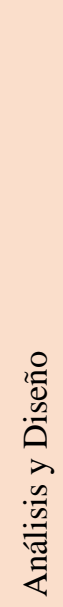 & 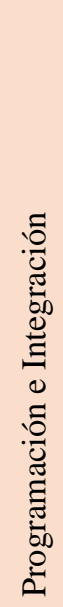 & 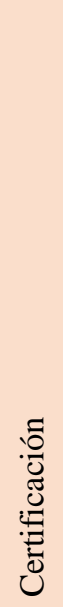 & 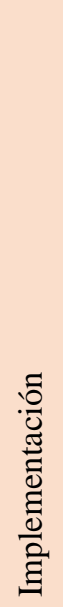 & $\stackrel{\circlearrowright}{\Xi}$ & 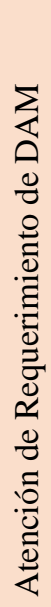 & 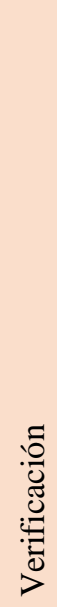 & $\begin{array}{l}\stackrel{\pi}{0} \\
\stackrel{0}{0} \\
\overline{0} \\
0\end{array}$ \\
\hline OE 1 & & $X$ & & & & & & & & & & & & & & & $\mathrm{X}$ & $\mathrm{X}$ & & & & & & & & & \\
\hline OE 2 & $\mathrm{X}$ & & & & & & & & & & & & & & & $\mathrm{X}$ & & & & & & & & & & & \\
\hline OE 3 & & & & & & & & & & & & & & & & & & $\mathrm{X}$ & & & & & & & $X$ & & \\
\hline OE 4 & $\mathrm{X}$ & $\mathrm{X}$ & & & & $\mathrm{X}$ & & & & & & & & & & & & & & & & & & & & & \\
\hline OE 5 & $\mathrm{X}$ & & & & & & & & & & & & & & & & & $X$ & $\mathrm{X}$ & X & $\mathrm{X}$ & $\mathrm{X}$ & $\mathrm{X}$ & $\mathrm{X}$ & $X$ & $\mathrm{X}$ & $\mathrm{X}$ \\
\hline
\end{tabular}

Nota: Se ha realizado el cruce entre los objetivos estratégicos de la empresa y sus procesos para poder identificar cuales están relacionados. Adaptado de "Manual de

Calidad 2017”, por El Objeto de Estudio, 2017. 


\section{(1)}

○ DIAGRAMA DE ACTTIVIDADES

○ SUBPROCESO DE “CONTROL Y SEGUIMIENTO DE PROYECTOS”

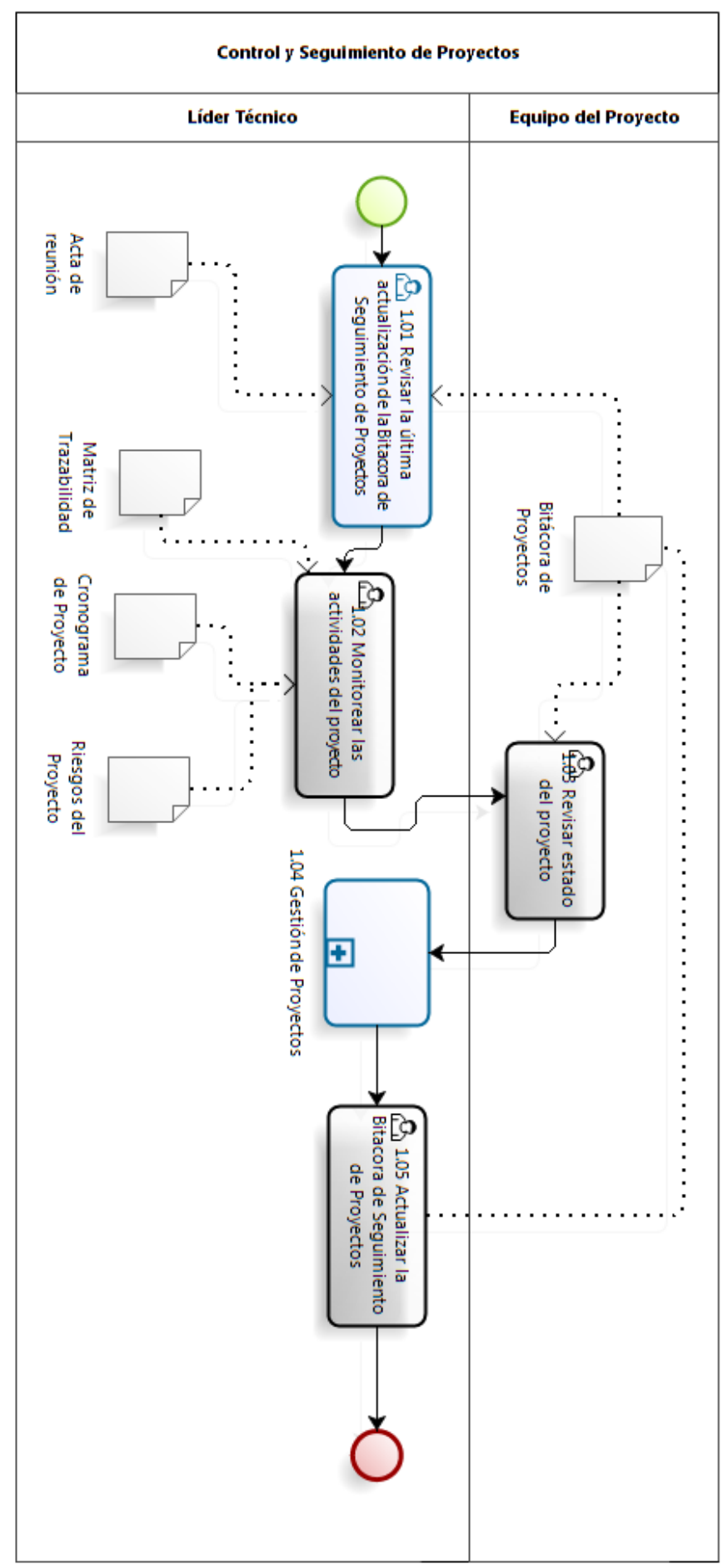

Figura 21. Diagrama de Actividades del Subproceso de Control y Seguimiento de Proyectos (TOBE). Adaptado de "Procedimiento de Gestión de Proyecto", por El Objeto de Estudio, 2017. 


\section{(1)}

○ SUBPROCESO DE “GESTIÓN DE PROYECTOS”

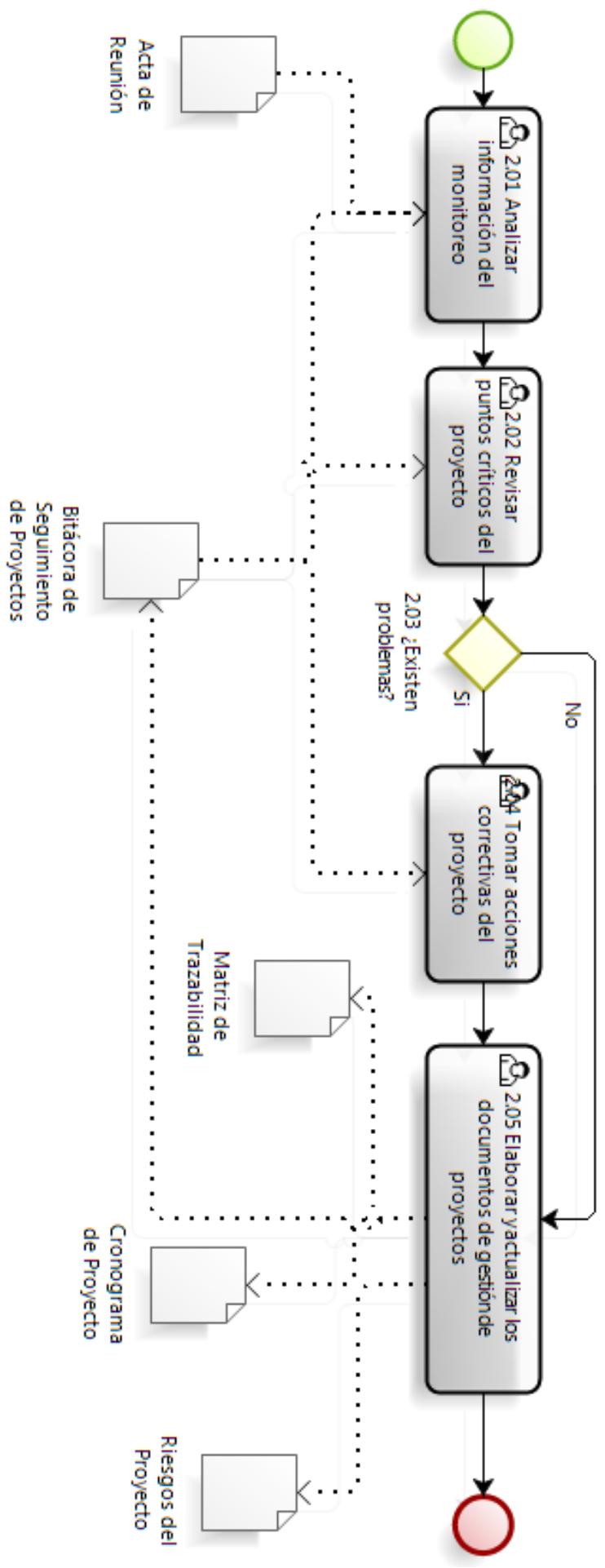

Figura 22. Diagrama de Actividades del Subproceso de Gestión de Proyectos (TOBE). Adaptado de "Procedimiento de Gestión de Proyecto", por El Objeto de Estudio, 2017. 


\section{(4)}

\section{○ PROCESOS DE NEGOCIO SELECCIONADO Y DESCRIPCIÓN}

El proceso seleccionado es Gestión de Proyectos el cual gestiona los requerimientos a lo largo del ciclo de vida del proyecto, mantiene la trazabilidad de los requerimientos y administra cambios en los requerimientos. También, monitorea las actividades, compromisos, riesgos, involucrados directos e indirectos del proyecto. Asimismo toma las acciones correctivas de acuerdo al seguimiento que se realiza del proyecto. Identifica riesgos a lo largo del proyecto, define estrategias, evalúa, prioriza y desarrolla los planes de mitigación y/o contingencia para afrontar el riesgo. Además, integra planes (actividades y recursos) coordina cruces y dependencias y realiza una coordinación integral. Por último, establece guías y criterios para tomar una decisión de acuerdo a las alternativas y métodos de evaluación definidos. Se divide en dos subprocesos:

\section{○ SUBPROCESO DE CSP}

Tabla 52

Actividades del Subprocesos de Control y Seguimiento de Proyectos (TOBE)

\begin{tabular}{|c|c|c|c|}
\hline $\mathbf{N}^{\mathbf{0}}$ & ACTIVIDAD & RESPONSABLE & $\begin{array}{c}\text { REGISTRO/ } \\
\text { DOCUMENTO }\end{array}$ \\
\hline 1.01 & $\begin{array}{l}\text { Revisar la última actualización de la Bitácora de } \\
\text { Seguimiento de Proyectos } \\
\text { Revisar los acuerdos, no conformidades, problemas, } \\
\text { lecciones aprendidas y mejoras registrados en la bitácora } \\
\text { de seguimiento de proyectos encontrados en la última } \\
\text { reunión de seguimiento del proyecto (se entiende interna) y } \\
\text { la externa con el cliente. } \\
\text { Cada uno de los puntos debe revisarse para conocer el } \\
\text { estado actual. } \\
\text { Periódicamente se deben realizar reuniones de seguimiento } \\
\text { del proyecto. }\end{array}$ & Líder técnico & $\begin{array}{l}\text { Acta de reunión / } \\
\text { Bitácora de } \\
\text { Seguimiento de } \\
\text { Proyectos }\end{array}$ \\
\hline 1.02 & $\begin{array}{l}\text { Monitorear las actividades del proyecto } \\
\text { - Los requerimientos del proyecto utilizando la Matriz de } \\
\text { Trazabilidad. } \\
\text { - Las variables del proyecto (plazo y esfuerzo), así como el } \\
\text { avance de actividades (realizadas por personal interno o } \\
\text { externo). Para este monitoreo utilizamos el cronograma de } \\
\text { proyecto. } \\
\text { - Los compromisos (hitos) del proyecto, para lo cual } \\
\text { utilizamos el cronograma de proyecto. }\end{array}$ & Líder técnico & $\begin{array}{c}\text { Sistema JIRA (Matriz } \\
\text { de Trazabilidad, } \\
\text { Cronograma del } \\
\text { Proyecto, Riesgos del } \\
\text { Proyectos) }\end{array}$ \\
\hline
\end{tabular}




\section{(9)}

- Los riesgos del proyecto deben monitorearse utilizando la plantilla Riesgos del Proyectos.

Las actividades del proyecto deben monitorearse de acuerdo a lo registrado en el Sistema JIRA.

1.03 Revisar el estado del proyecto

Revisar el estado de cada actividad, documento, entregable, riesgo, compromiso, etc. Asimismo identificar todo problema o suceso que pueda impactar el proyecto para poder ser reportado al Gerente de Producto.

Nota: El equipo de trabajo debe transmitir sus inquietudes o sus necesidades.

\subsection{Gestionar el proyecto}

Realizar la Gestión del Proyecto utilizando la información recolectada durante las actividades 1.02 y 1.03 .

Para ello debemos seguir el Proceso No2 Gestión de Proyectos.

\subsection{Actualizar la Bitácora de seguimiento de proyectos}

Actualizar los acuerdos, mejoras, no conformidades, problemas, lecciones aprendidas y mejoras en la Bitácora de seguimiento de proyectos.

Equipo de Proyecto

Sistema JIRA

(Bitácora de

Seguimiento de

Proyectos)

Líder técnico

\section{Líder técnico}

Sistema JIRA

(Bitácora de

Seguimiento de

Proyectos)

La bitácora de seguimiento de proyectos debe ser entregada al Gerente de Producto para su revisión.

Nota: Se ha descrito las actividades, responsable y registro/documento del subproceso de Control y Seguimiento de Proyectos. Adaptado de "Procedimiento de Gestión de Proyecto", por El Objeto de Estudio, 2017.

\section{○ SUBPROCESO DE GESTIÓN DE PROYECTOS}

Tabla 53

Actividades del Subprocesos de Gestión de Proyectos (TOBE)

\begin{tabular}{|l|l|c|c|}
\hline $\mathbf{N}^{\mathbf{2}}$ & \multicolumn{1}{|c|}{ ACTIVIDAD } & RESPONSABLE & \multicolumn{1}{|c|}{$\begin{array}{c}\text { REGISTRO/ } \\
\text { DOCUMENTO }\end{array}$} \\
\hline 2.01 & $\begin{array}{l}\text { Analizar la información obtenida durante la reunión de } \\
\text { seguimiento de proyecto. } \\
\text { Este análisis permitirá tomar las acciones correctivas } \\
\text { necesarias. } \\
\text { Nota: Los acuerdos, problemas, no conformidades } \\
\text { (acción correctiva), entre otros, se debe de colocar en la }\end{array}$ & $\begin{array}{c}\text { Acta de reunión / } \\
\text { Sistema JIRA (Bitácora } \\
\text { de Seguimiento de }\end{array}$ \\
\hline
\end{tabular}




\section{$(\boldsymbol{1})$}

\begin{tabular}{|c|c|c|c|}
\hline & $\begin{array}{l}\text { bitácora de seguimiento de proyectos, en caso sea } \\
\text { necesario se debe generar un acta de la reunión. }\end{array}$ & & \\
\hline 2.02 & $\begin{array}{l}\text { Revisar si el proyecto posee acciones correctivas } \\
\text { pendientes de cierre. Estas acciones correctivas } \\
\text { pendientes de cierre deben estar anotadas en la Bitácora } \\
\text { de Seguimiento de Proyectos para conocer el estado de } \\
\text { avance de cada acción correctiva. }\end{array}$ & Líder técnico & $\begin{array}{c}\text { Sistema JIRA (Bitácora } \\
\text { de Seguimiento de } \\
\text { Proyectos) }\end{array}$ \\
\hline 2.03 & $\begin{array}{l}\text { ¿Existen problemas? } \\
\text { Si existen problemas dentro del proyecto se debe proceder } \\
\text { a la actividad 2.04. En caso contrario, realizar la actividad } \\
2.05 \text {. }\end{array}$ & Líder técnico & \\
\hline 2.04 & $\begin{array}{l}\text { Tomar acciones correctivas } \\
\text { Para ello en la Bitácora de seguimiento de Proyectos por } \\
\text { cada problema encontrado debemos: } \\
\text { - Anotar el problema suscitado. } \\
\text { - Anotar las acciones que se tomarán para su } \\
\text { solución }\end{array}$ & Líder técnico & $\begin{array}{c}\text { Sistema JIRA (Bitácora } \\
\text { de Seguimiento de } \\
\text { Proyectos) }\end{array}$ \\
\hline 2.05 & $\begin{array}{l}\text { Debemos elaborar o actualizar los entregables de gestión } \\
\text { del proyecto en el Sistema JIRA, los cuales son: } \\
\text { - Matriz de Trazabilidad } \\
\text { - Cronograma de Proyecto } \\
\text { - Riesgos del Proyecto } \\
\text { - Bitácora de Seguimiento de Proyectos } \\
\text { Estos documentos son actualizados con la información } \\
\text { recolectada durante la reunión de seguimiento. Asimismo, } \\
\text { son presentados al Gerente de Producto para su revisión. } \\
\text { Nota: El Gerente de Producto de acuerdo a la } \\
\text { información revisada debe escalar los problemas y hechos } \\
\text { que crea conveniente tanto al Gerente de Operaciones } \\
\text { como al Sponsor del Proyecto. }\end{array}$ & Líder técnico & $\begin{array}{l}\text { Sistema JIRA (Matriz } \\
\text { de Trazabilidad, } \\
\text { Cronograma del } \\
\text { Proyecto, Riesgos del } \\
\text { Proyectos, Bitácora de } \\
\text { Seguimiento de } \\
\text { Proyectos) }\end{array}$ \\
\hline
\end{tabular}

Nota: Se ha descrito las actividades, responsable y registro/documento del subproceso de Gestión de Proyectos.

Adaptado de "Procedimiento de Gestión de Proyecto", por El Objeto de Estudio, 2017. 


\section{(4)}

○ ROLES DE NEGOCIO: MATRIZ RACI

○ Subproceso de Control y Seguimiento de Proyectos

Tabla 54

Matriz RACI - Subproceso de CSP (TOBE)

\begin{tabular}{|c|c|c|c|c|c|}
\hline Matriz RACI & \multicolumn{5}{|c|}{ ROLES } \\
\hline $\begin{array}{l}\text { R: Responsable } \\
\text { A: Aprobado } \\
\text { C: Consultado } \\
\text { I: Informado }\end{array}$ & $\begin{array}{l}\text { Gerente de } \\
\text { operaciones }\end{array}$ & $\begin{array}{l}\text { Asistente } \\
\text { de } \\
\text { Sistemas }\end{array}$ & $\begin{array}{l}\text { Gerente } \\
\text { de área }\end{array}$ & $\begin{array}{l}\text { Líder } \\
\text { técnico }\end{array}$ & $\begin{array}{l}\underline{\text { Equipo }} \\
\underline{\text { de }} \\
\text { Proyecto }\end{array}$ \\
\hline \multicolumn{6}{|l|}{ Actividades } \\
\hline $\begin{array}{l}\text { Revisar la última actualización de la Bitácora de } \\
\text { Seguimiento de Proyectos. }\end{array}$ & I & $\mathrm{C}$ & I & $\mathrm{R}$ & $\mathrm{C}$ \\
\hline Monitorear las actividades del proyecto & I & $\mathrm{C}$ & I & $\mathrm{R}$ & $\mathrm{C}$ \\
\hline Revisar el estado del proyecto & I & $\mathrm{C}$ & I & $\mathrm{R}$ & $\mathrm{C}$ \\
\hline Gestionar el proyecto & I & $\mathrm{C}$ & I & $\mathrm{R}$ & $\mathrm{C}$ \\
\hline Actualizar la Bitácora de seguimiento de proyectos & I & $\mathrm{C}$ & I & $\mathrm{R}$ & $\mathrm{C}$ \\
\hline
\end{tabular}

Nota: Se ha descrito las actividades y roles del subproceso de Control y Seguimiento de Proyectos. Adaptado de "Procedimiento de Gestión de Proyecto", por El Objeto de Estudio, 2017.

\section{○ Subproceso de Gestión de Proyectos}

Tabla 55

Matriz RACI - Subproceso de Gestión de Proyectos (TOBE)

\begin{tabular}{|c|c|c|c|c|c|}
\hline Matriz RACI & \multicolumn{5}{|c|}{ ROLES } \\
\hline $\begin{array}{l}\text { R: Responsable } \\
\text { A: Aprobado } \\
\text { C: Consultado } \\
\text { I: Informado }\end{array}$ & $\begin{array}{l}\text { Gerente de } \\
\text { operaciones }\end{array}$ & $\begin{array}{l}\text { Asistente } \\
\text { de } \\
\text { Sistemas }\end{array}$ & $\begin{array}{l}\text { Gerente } \\
\text { de área }\end{array}$ & $\begin{array}{l}\text { Líder } \\
\text { técnico }\end{array}$ & $\begin{array}{c}\text { Equipo } \\
\text { de } \\
\text { Proyecto }\end{array}$ \\
\hline \multicolumn{6}{|l|}{ Actividades } \\
\hline $\begin{array}{l}\text { Analizar la información obtenida durante la reunión } \\
\text { de seguimiento de proyecto. }\end{array}$ & I & $\mathrm{C}$ & I & $\mathrm{R}$ & $\mathrm{C}$ \\
\hline $\begin{array}{l}\text { Revisar si el proyecto posee acciones correctivas } \\
\text { pendientes de cierre. }\end{array}$ & I & $\mathrm{C}$ & I & $\mathrm{R}$ & $\mathrm{C}$ \\
\hline Verificar si existen problemas & I & $\mathrm{C}$ & I & $\mathrm{R}$ & $\mathrm{C}$ \\
\hline Tomar acciones correctivas & A & $\mathrm{C}$ & A & $\mathrm{R}$ & $\mathrm{C}$ \\
\hline $\begin{array}{l}\text { Elaborar o actualizar los documentos de gestión del } \\
\text { proyecto }\end{array}$ & A & $\mathrm{C}$ & A & $\mathrm{R}$ & $\mathrm{C}$ \\
\hline
\end{tabular}

Nota: Se ha descrito las actividades y roles del subproceso de Gestión de Proyectos. Adaptado de

"Procedimiento de Gestión de Proyecto", por El Objeto de Estudio, 2017. 


\section{ARQUITECTURA DE DATOS}

\section{MODELO DE DATOS}

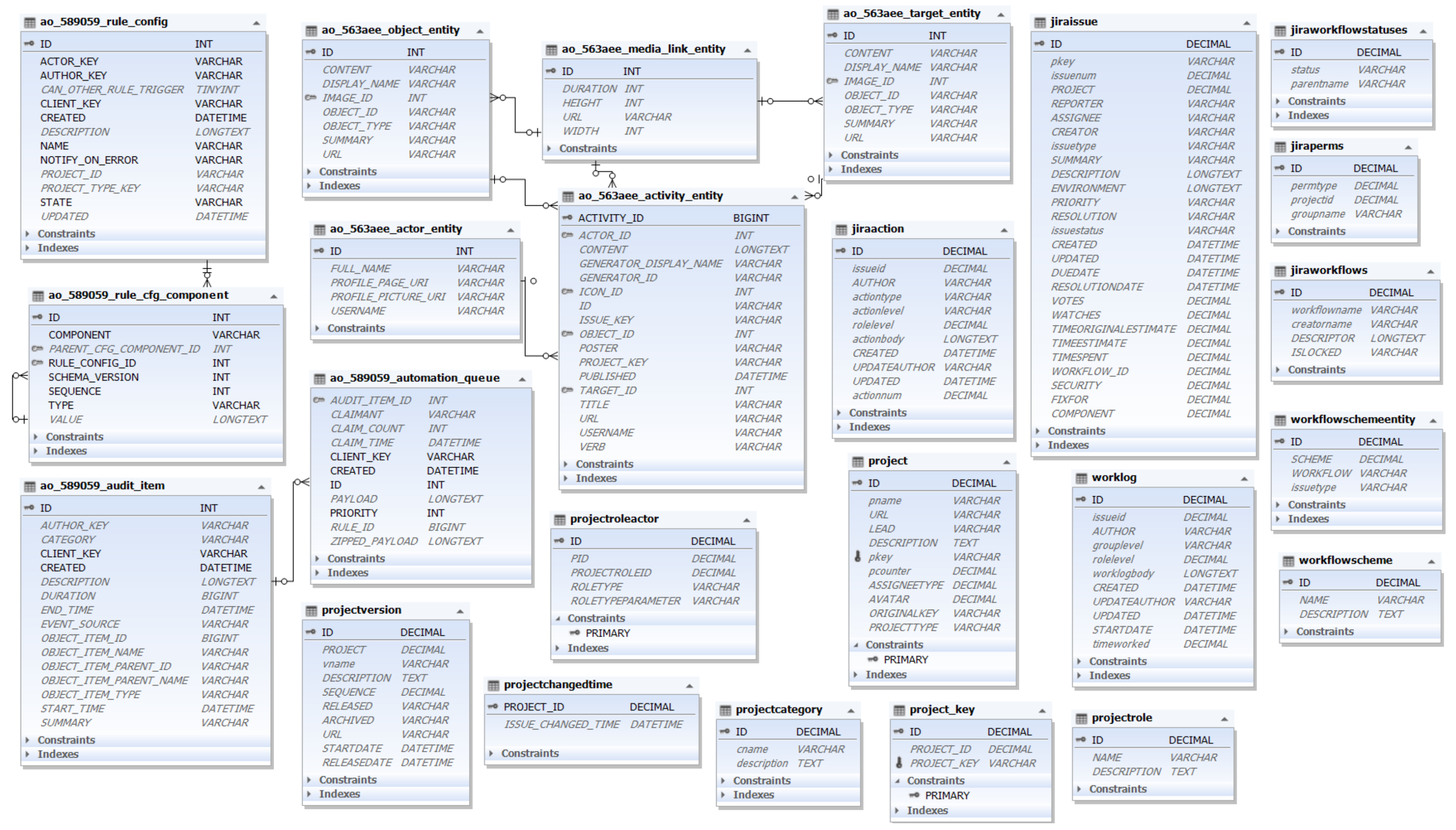

Figura 23. Modelo de Datos (TOBE). Elaboración propia 


\section{(4)}

$\bigcirc$

\section{DICCIONARIO DE DATOS}

Tabla 56

Tabla jiraaction

\begin{tabular}{|l|l|}
\hline TABLA & jiraaction \\
\hline Descripción & Información sobre la acción en el jira \\
\hline ATRIBUTO & Descripción \\
\hline ID & Identificador de la tabla \\
\hline issueid & Identificador de la incidencia \\
\hline AUTHOR & Nombre de autor \\
\hline actiontype & Tipo de acción \\
\hline actionlevel & Nivel de acción \\
\hline rolelevel & Nivel de rol \\
\hline actionbody & Cuerpo de acción \\
\hline CREATED & Fecha creada \\
\hline UPDATEAUTHOR & Autor de actualización \\
\hline UPDATED & Fecha actualizada \\
\hline actionnum & Número de acción \\
\hline
\end{tabular}

Nota: Se ha descrito cada atributo de la tabla jiraaction. Elaboración propia

Tabla 57

Tabla jiraissue

\begin{tabular}{|l|l|}
\hline TABLA & jiraissue \\
\hline Descripción & Información sobre el jira \\
\hline ATRIBUTO & Descripción \\
\hline ID & Identificador de la tabla \\
\hline pkey & Identificador del proyecto \\
\hline issuenum & Número de incidencia \\
\hline PROJECT & Número de proyecto \\
\hline REPORTER & Nombre del informador \\
\hline ASSIGNEE & Nombre del asignado \\
\hline CREATOR & Nombre del creador \\
\hline issuetype & Tipo de incidencia \\
\hline SUMMARY & Resumen de la incidencia \\
\hline DESCRIPTION & Descripción de la incidencia \\
\hline ENVIRONMENT & Ambiente de la incidencia \\
\hline PRIORITY & Prioridad de la incidencia \\
\hline RESOLUTION & Resolución de la incidencia \\
\hline issuestatus & Estado de la incidencia \\
\hline CREATED & Fecha de creación \\
\hline UPDATED & Fecha de actualización \\
\hline DUEDATE & Fecha de vencimiento \\
\hline RESOLUTIONDATE & Fecha de resolución \\
\hline
\end{tabular}




\section{()}

\begin{tabular}{|l|l|}
\hline VOTES & Número de votos \\
\hline WATCHES & Número de vigilantes \\
\hline TIMEORIGINALESTIMATE & Tiempo original estimado \\
\hline TIMEESTIMATE & Tiempo estimado actual \\
\hline TIMESPENT & Tiempo gastado \\
\hline WORKFLOW_ID & Identificador del flujo de trabajo \\
\hline SECURITY & Seguridad \\
\hline FIXFOR & Número de arreglos \\
\hline COMPONENT & Número de componentes \\
\hline
\end{tabular}

Nota: Se ha descrito cada atributo de la tabla jiraissue. Elaboración propia

Tabla 58

Tabla jiraworkflows

\begin{tabular}{|l|l|}
\hline TABLA & jiraworkflows \\
\hline Descripción & Información sobre el flujo de trabajo del jira \\
\hline ATRIBUTO & Descripción \\
\hline ID & Identificador de la tabla \\
\hline workflowname & Nombre de flujo \\
\hline creatorname & Nombre de creador \\
\hline DESCRIPTOR & Descripción \\
\hline ISLOCKED & Indicador de Bloqueado \\
\hline
\end{tabular}

Nota: Se ha descrito cada atributo de la tabla jiraworkflows. Elaboración propia

Tabla 59

Tabla jiraworkflowstatuses

\begin{tabular}{|l|l|}
\hline TABLA & jiraworkflowstatuses \\
\hline Descripción & Información sobre el estado del flujo de trabajo del jira \\
\hline ATRIBUTO & Descripción \\
\hline ID & Identificador de la tabla \\
\hline status & Nombre de estado \\
\hline parentname & Nombre del padre \\
\hline
\end{tabular}

Nota: Se ha descrito cada atributo de la tabla jiraworkflowstatuses. Elaboración propia

Tabla 60

Tabla project_key

\begin{tabular}{|l|l|}
\hline TABLA & project_key \\
\hline Descripción & Información sobre la llave del proyecto \\
\hline ATRIBUTO & Descripción \\
\hline ID & Identificador de la tabla \\
\hline PROJECT_ID & Identificador de proyecto \\
\hline
\end{tabular}




\section{(1)}

PROJECT_KEY

Llave de proyecto

Nota: Se ha descrito cada atributo de la tabla project_key. Elaboración propia

Tabla 61

Tabla project

\begin{tabular}{|l|l|}
\hline TABLA & project \\
\hline Descripción & Información sobre el proyecto \\
\hline ATRIBUTO & Descripción \\
\hline ID & Identificador de la tabla \\
\hline pname & Nombre de proyecto \\
\hline URL & URL de proyecto \\
\hline LEAD & Nombre de lider \\
\hline DESCRIPTION & Descripción del proyecto \\
\hline pkey & Llave del proyecto \\
\hline pcounter & Tipo de asignación \\
\hline ASSIGNEETYPE & Avatar \\
\hline AVATAR & Llave original \\
\hline ORIGINALKEY & Tipo de proyecto \\
\hline PROJECTTYPE & \\
\hline
\end{tabular}

Nota: Se ha descrito cada atributo de la tabla project. Elaboración propia

Tabla 62

Tablaao_563aee_actor_entity

\begin{tabular}{|l|l|}
\hline TABLA & ao_563aee_actor_entity \\
\hline Descripción & Información sobre la entidad actor \\
\hline ATRIBUTO & Descripción \\
\hline ID & Identificador del proyecto línea base \\
\hline FULL_NAME & Nombre completo \\
\hline PROFILE_PAGE_URI & Página URI del perfil \\
\hline PROFILE_PICTURE_URI & Imágen URI del perfil \\
\hline USERNAME & Nombre de usuario \\
\hline
\end{tabular}

Nota: Se ha descrito cada atributo de la tabla ao_563aee_actor_entity. Elaboración propia

Tabla 63

Tabla projectchangedtime

\begin{tabular}{|l|l|}
\hline TABLA & projectchangedtime \\
\hline Descripción & Información sobre un cambio del proyecto \\
\hline ATRIBUTO & Descripción \\
\hline PROJECT_ID & Identificador de la tabla \\
\hline ISSUE_CHANGED_TIME & Fecha de cambio de incidencia \\
\hline
\end{tabular}

Nota: Se ha descrito cada atributo de la tabla projectchangedtime. Elaboración propia 


\section{()}

Tabla 64

Tabla projectversion

\begin{tabular}{|l|l|}
\hline TABLA & projectversion \\
\hline Descripción & Información sobre la versión del proyecto \\
\hline ATRIBUTO & Descripción \\
\hline ID & Identificador de la tabla \\
\hline PROJECT & Fecha de cambio de incidencia \\
\hline vname & Nombre de la versión \\
\hline DESCRIPTION & Descripción de la versión \\
\hline SEQUENCE & Número de secuencia \\
\hline RELEASED & Liberado \\
\hline ARCHIVED & Archivado \\
\hline URL & URL de versión \\
\hline STARTDATE & Fecha de inicio \\
\hline RELEASEDATE & Fecha de liberación \\
\hline
\end{tabular}

Nota: Se ha descrito cada atributo de la tabla projectversion. Elaboración propia

Tabla 65

Tabla projectroleactor

\begin{tabular}{|l|l|}
\hline TABLA & projectroleactor \\
\hline Descripción & Información sobre el actor del proyecto \\
\hline ATRIBUTO & Descripción \\
\hline ID & Identificador de la tabla \\
\hline PID & Identificador de proyecto \\
\hline PROJECTROLEID & Identificador de rol del proyecto \\
\hline ROLETYPE & Tipo de rol \\
\hline ROLETYPEPARAMETER & Tipo de rol paramétrico \\
\hline
\end{tabular}

Nota: Se ha descrito cada atributo de la tabla projectroleactor. Elaboración propia

Tabla 66

Tabla projectrole

\begin{tabular}{|l|l|}
\hline TABLA & projectrole \\
\hline Descripción & Información sobre el rol del proyecto \\
\hline ATRIBUTO & Descripción \\
\hline ID & Identificador de la tabla \\
\hline NAME & Nombre del rol del proyecto \\
\hline DESCRIPTION & Descripción del rol del proyecto \\
\hline
\end{tabular}

Nota: Se ha descrito cada atributo de la tabla projectrole. Elaboración propia 


\section{()}

Tabla 67

Tabla worklog

\begin{tabular}{|l|l|}
\hline TABLA & worklog \\
\hline Descripción & Información sobre la bitácora de trabajo \\
\hline ATRIBUTO & Descripción \\
\hline ID & Identificador de la tabla \\
\hline issueid & Identificador de incidencia \\
\hline AUTHOR & Nombre de autor \\
\hline grouplevel & Nivel de grupo \\
\hline rolelevel & Nivel de rol \\
\hline worklogbody & Cuerpo de la bitacora de trabajo \\
\hline CREATED & Fecha creada \\
\hline UPDATEAUTHOR & Autor de actualización \\
\hline UPDATED & Fecha de actualizada \\
\hline STARTDATE & Fecha de inicio \\
\hline timeworked & Tiempo trabajado \\
\hline
\end{tabular}

Nota: Se ha descrito cada atributo de la tabla worklog. Elaboración propia

Tabla 68

Tabla workflowscheme

\begin{tabular}{|l|l|}
\hline TABLA & workflowscheme \\
\hline Descripción & Información sobre el esquema del flujo de trabajo \\
\hline ATRIBUTO & Descripción \\
\hline ID & Identificador de la tabla \\
\hline NAME & Nombre del esquema \\
\hline DESCRIPTION & Descripción del esquema \\
\hline
\end{tabular}

Nota: Se ha descrito cada atributo de la tabla workflowscheme. Elaboración propia

Tabla 69

Tabla workflowschemeentity

\begin{tabular}{|l|l|}
\hline TABLA & workflowschemeentity \\
\hline Descripción & Información sobre el esquema entidad del flujo de trabajo \\
\hline ATRIBUTO & Descripción \\
\hline ID & Identificador de la tabla \\
\hline SCHEME & Número del esquema \\
\hline WORKFLOW & Flujo de trabajo \\
\hline issuetype & Tipo de incidencia \\
\hline
\end{tabular}

Nota: Se ha descrito cada atributo de la tabla workflowschemeentity. Elaboración propia 


\section{(1)}

\section{○ MATRIZ DE ENTIDADES DE DATOS VS PROCESOS DEL NEGOCIO}

Tabla 70

Matriz de Entidades de Datos vs Procesos del Negocio (TOBE)

\begin{tabular}{|c|c|c|c|c|c|c|c|c|c|c|c|c|c|c|c|c|c|c|c|c|c|c|c|c|c|c|c|}
\hline \multirow{3}{*}{ Entidades de Datos } & \multicolumn{27}{|c|}{ PROCESOS } \\
\hline & \multicolumn{3}{|c|}{ Estratégicos } & \multicolumn{13}{|c|}{ Apoyo } & \multicolumn{11}{|c|}{ Claves } \\
\hline & 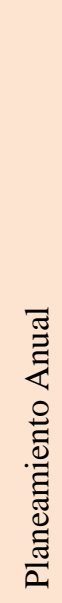 & 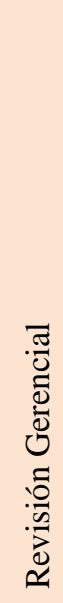 & 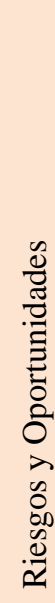 & 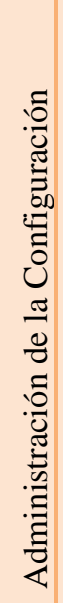 & 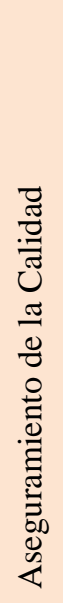 & 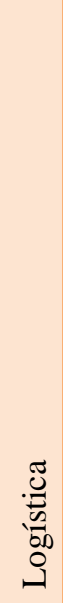 & 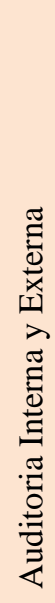 & 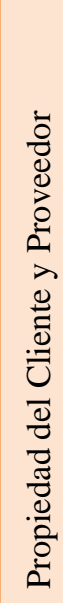 & 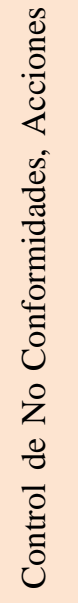 & 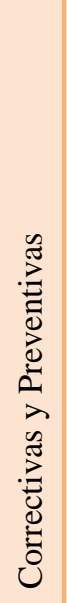 & 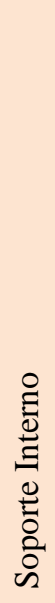 & $\begin{array}{l}0 \\
0 \\
0 \\
0 \\
0 \\
0 \\
0 \\
0 \\
0 \\
0 \\
0 \\
0 \\
0 \\
0 \\
0 \\
0 \\
0 \\
0 \\
0\end{array}$ & 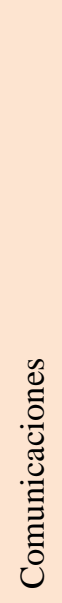 & 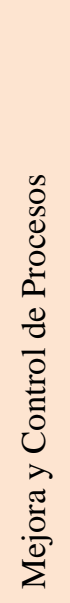 & 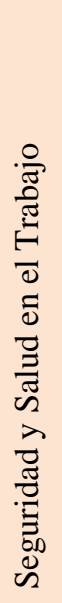 & 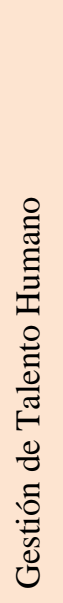 & 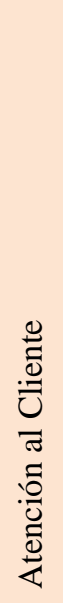 & 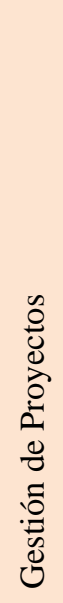 & 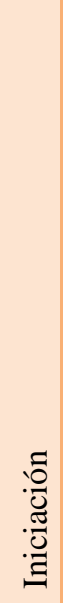 & 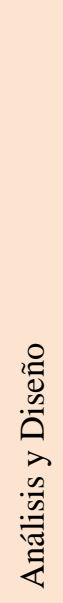 & 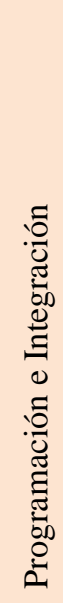 & 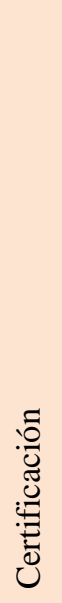 & 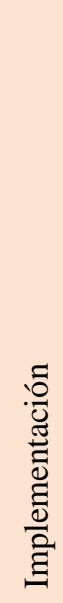 & Ü. & 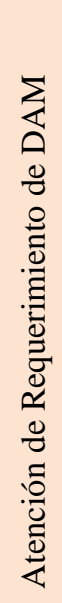 & 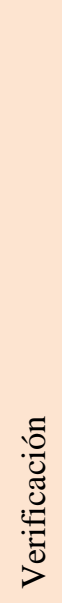 & $\frac{\pi}{\tilde{0}}$ \\
\hline ao_589059_rule_config & & & & & & & & & & & & & & & & & & $\mathrm{X}$ & & & & & & & & & \\
\hline ao_563aee_target_entity & & & & & & & & & & & & & & & & & & $\mathrm{X}$ & & & & & & & & & \\
\hline ao_563aee_actor_entity & & & & & & & & & & & & & & & & & & $\mathrm{X}$ & & & & & & & & & \\
\hline
\end{tabular}




\section{(U)}

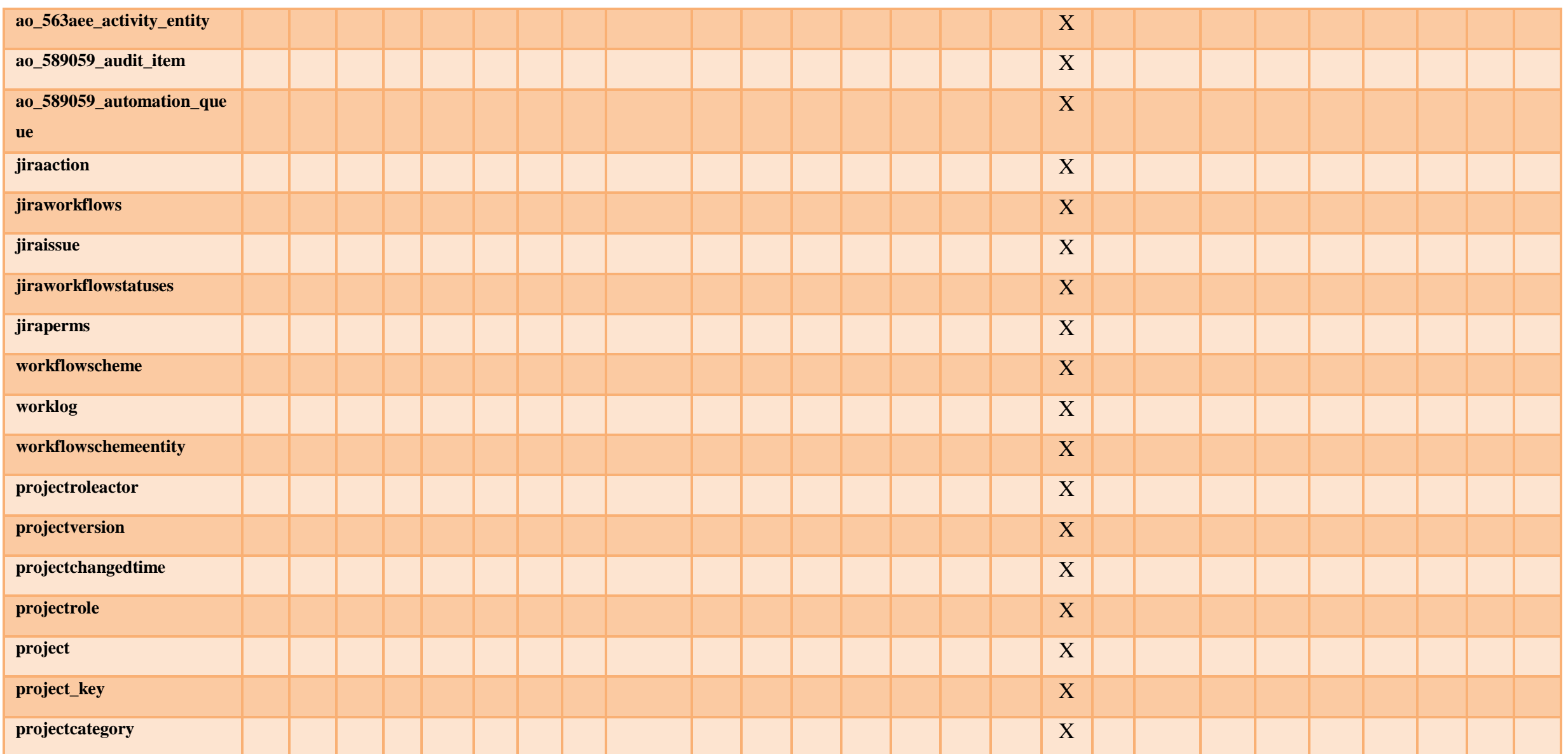

Nota: Se ha realizado el cruce entre tablas y procesos para poder identificar cuales están relacionados. Adaptado de "Manual de Configuración", por El Objeto de Estudio, 2017. 


\section{(1)}

\section{ARQUITECTURA DE APLICACIÓN}

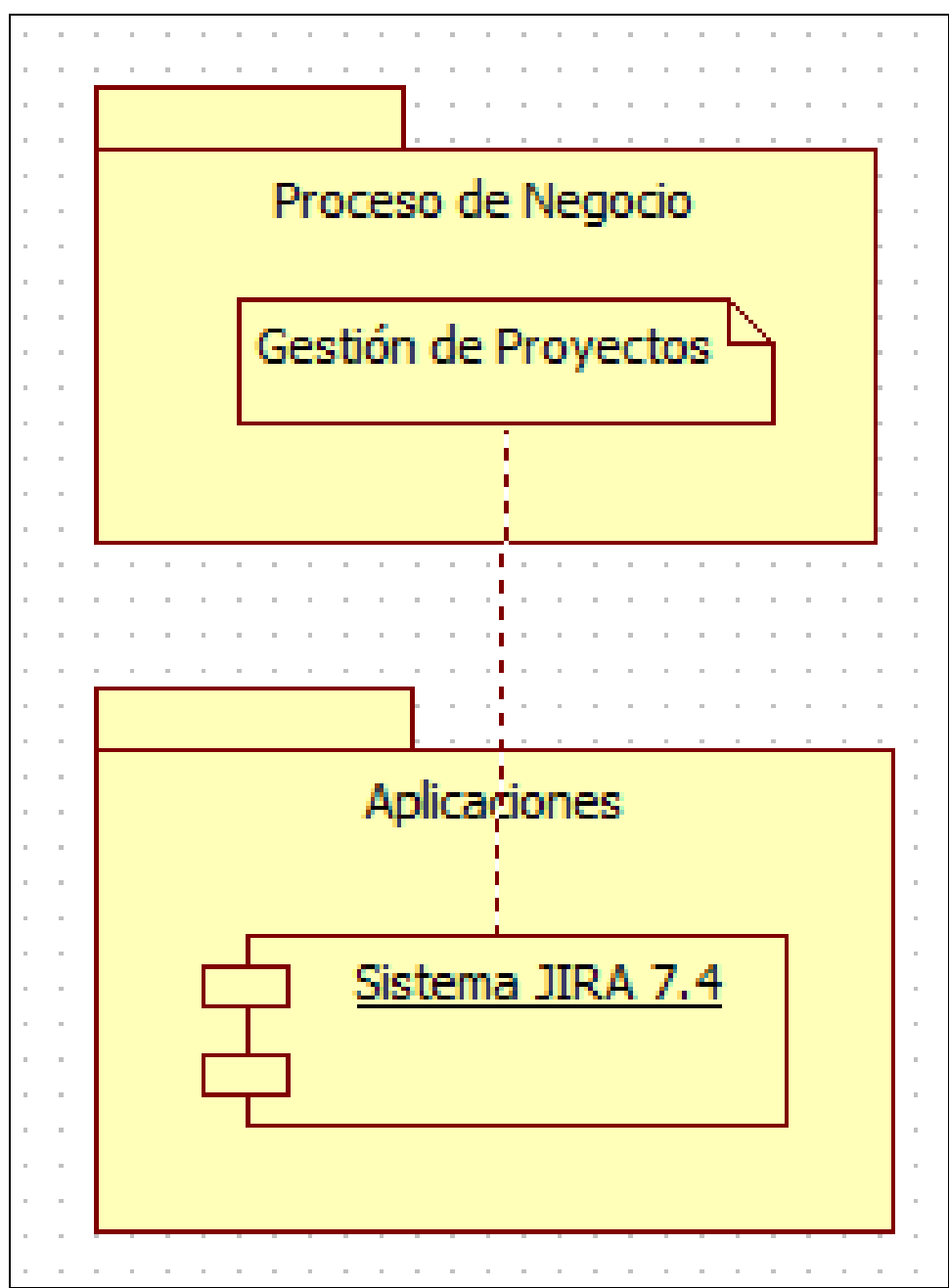

Figura 24. Diagrama de Aplicaciones del Proceso de Gestión de Proyectos (TOBE). Elaboración propia.

Tabla 71

Detalle de Aplicaciones del Proceso Gestión de Proyectos (TOBE)

\begin{tabular}{|l|l|l|}
\hline Proceso de Negocio & Aplicación & Descripción \\
\hline Gestión de Proyectos & Sistema JIRA & El sistema JIRA servirá para llevar un mejor \\
& control en el seguimiento de actividades de los \\
& proyectos, los involucrados podrán llenar sus \\
& horas de sus tareas asignadas y el Líder técnico \\
& podrá realizar una gestión más óptima y en tiempo \\
& real.
\end{tabular}

Nota: Se ha descrito las aplicaciones existentes del proceso de gestión de proyectos. Elaboración propia. 


\section{(1)}

4. ARQUITECTURA TECNOLÓGICA

- COMPONENTES DE TECNOLOGÍA Y SUS RELACIONES CON LOS SISTEMAS INFORMACIÓN

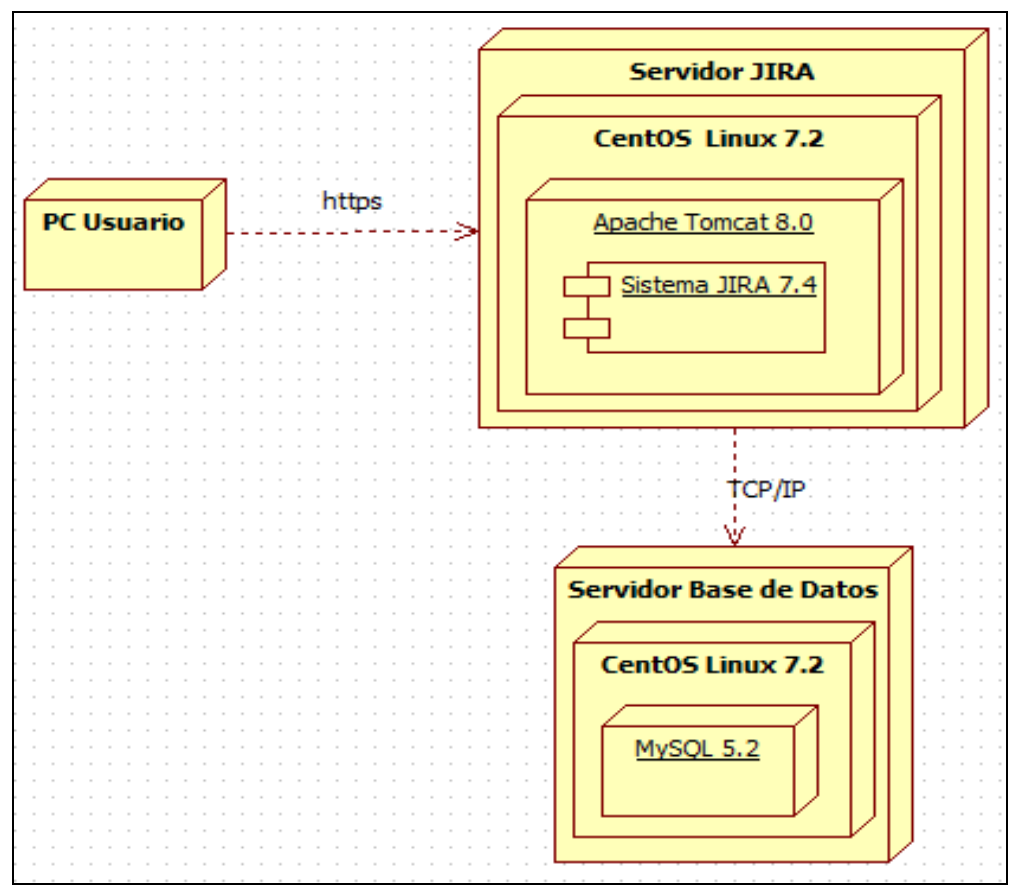

Figura 25. Diagrama de Componentes del Proceso de Gestión de Proyectos (TOBE). Elaboración propia.

\section{○ PlATAFORMA DE TECNOLOGÍA Y SU DESCOMPOSICIÓN}

Tabla 72

Detalle de Componentes del Proceso Gestión de Proyectos (TOBE)

\begin{tabular}{|l|l|}
\hline \multicolumn{2}{|l|}{ SISTEMA JIRA } \\
\hline Servidor & Servidor 2012 \\
\hline Sistema Operativo & CentOS Linux 7.2 \\
\hline Contenedor & Apache Tomcat 8.0 \\
\hline BASE DE DATOS & \\
\hline Servidor & Servidor de base de datos \\
\hline Sistema Operativo & CentOS Linux 7.2 \\
\hline Base de datos & MySQL 5.2 \\
\hline
\end{tabular}

Nota: Se ha descrito el servidor, sistema operativo y disco duro por cada aplicación. Elaboración propia. 


\section{(4)}

\section{○ AMBIENTES Y UBICACIONES}

Tabla 73

Detalle de Ubicaciones y Ambientes (TOBE)

\begin{tabular}{|c|c|c|c|}
\hline UBICACIÓN & AMBIENTES & SERVIDORES & DETALLE \\
\hline \multirow[t]{4}{*}{ PISO 1} & Recepción & & \\
\hline & Directorio & & \\
\hline & $\begin{array}{c}\text { Gerencia de Investigación } \\
\text { y Desarrollo }\end{array}$ & & \\
\hline & Desarrollo V & Servidor EFT & $\begin{array}{l}\text { Tipo: Físico } \\
\text { Procesador: Supermicro } \\
\text { Aplicaciones: Oracle Linux } \\
\text { Discos:3 } \\
\text { Capacidad: 160G, 2T, } 2 \mathrm{~T} \\
\text { Potencia } 350 \mathrm{~W}\end{array}$ \\
\hline \multirow[t]{6}{*}{ PISO 2} & Gerencia de Soporte & Servidor RSA & $\begin{array}{l}\text { Tipo: Físico } \\
\text { Procesador: Intel } \\
\text { Aplicaciones: AIX P520 } \\
\text { Discos:1 } \\
\text { Capacidad: 1T } \\
\text { Potencia 350W }\end{array}$ \\
\hline & & Servidor Soporte & $\begin{array}{l}\text { Tipo: Físico } \\
\text { Procesador: Intel } \\
\text { Aplicaciones: Oracle Linux } \\
\text { Discos:2 } \\
\text { Capacidad: } 500 \mathrm{G}, 1 \mathrm{~T} \\
\text { Potencia } 350 \mathrm{~W}\end{array}$ \\
\hline & Control & Servidor Certificación & $\begin{array}{l}\text { Tipo: Físico } \\
\text { Procesador: Supermicro } \\
\text { Aplicaciones: Oracle Linux } \\
\text { Discos:3 } \\
\text { Capacidad: 1T, 1T, 1T } \\
\text { Potencia 350W }\end{array}$ \\
\hline & & Servidor de Control & $\begin{array}{l}\text { Tipo: Físico } \\
\text { Procesador: Intel } \\
\text { Aplicaciones: Server } 2012 \\
\text { Discos:2 } \\
\text { Capacidad: 2T, 2T } \\
\text { Potencia 350W }\end{array}$ \\
\hline & Gerencia de Marketing & Servidor Marketing & $\begin{array}{l}\text { Tipo: Físico } \\
\text { Procesador: Intel } \\
\text { Aplicaciones: Server2008 } \\
\text { Discos:1 } \\
\text { Capacidad: } 2 \mathrm{~T} \\
\text { Potencia 350W }\end{array}$ \\
\hline & Gerencia de CAT 01 & Servidor CAT 1 & $\begin{array}{l}\text { Tipo: Físico } \\
\text { Procesador: Supermicro } \\
\text { Aplicaciones: Oracle Linux }\end{array}$ \\
\hline
\end{tabular}




\begin{tabular}{|c|c|c|c|}
\hline UBICACIÓN & AMBIENTES & SERVIDORES & DETALLE \\
\hline \multirow{9}{*}{ PISO 3} & & & $\begin{array}{l}\text { Discos:3 } \\
\text { Capacidad: 1T, 2T, 2T } \\
\text { Potencia 350W }\end{array}$ \\
\hline & Gerencia de CAT 02 & Servidor SPA & $\begin{array}{l}\text { Tipo: Físico } \\
\text { Procesador: Supermicro } \\
\text { Aplicaciones: Oracle Linux } \\
\text { Discos:2 } \\
\text { Capacidad: 1T, 1T } \\
\text { Potencia 350W }\end{array}$ \\
\hline & \multirow[t]{7}{*}{ Sala de servidores } & Servidor Antispam & $\begin{array}{l}\text { Tipo: Físico } \\
\text { Procesador: Intel } \\
\text { Aplicaciones: Linux } 6.5 \\
\text { Discos:1 } \\
\text { Capacidad: 160G } \\
\text { Potencia 350W }\end{array}$ \\
\hline & & Servidor Exchange & $\begin{array}{l}\text { Tipo: Físico } \\
\text { Procesador: Intel } \\
\text { Aplicaciones: Windows Server } 2003 \\
\text { Discos:1 } \\
\text { Capacidad: 1T } \\
\text { Potencia 350W }\end{array}$ \\
\hline & & Servidor Firewall & $\begin{array}{l}\text { Tipo: Físico } \\
\text { Procesador: Intel } \\
\text { Aplicaciones: Linux } 6.5 \\
\text { Discos:1 } \\
\text { Capacidad: 1T } \\
\text { Potencia 350W }\end{array}$ \\
\hline & & Servidor 2008 & $\begin{array}{l}\text { Tipo: Físico } \\
\text { Procesador: Intel } \\
\text { Aplicaciones: Server } 2008 \\
\text { Discos:1 } \\
\text { Capacidad: 1T } \\
\text { Potencia 350W }\end{array}$ \\
\hline & & Servidor IBM P720 & $\begin{array}{l}\text { Tipo: Físico } \\
\text { Procesador: IBM } \\
\text { Aplicaciones: AIX } 720 \\
\text { Discos:1 } \\
\text { Capacidad: 600G } \\
\text { Potencia 350W }\end{array}$ \\
\hline & & Servidor 2012 & $\begin{array}{l}\text { Tipo: Físico } \\
\text { Procesador: Intel } \\
\text { Aplicaciones: Server } 2012 \\
\text { Discos: } 1 \\
\text { Capacidad: } 2 \mathrm{~T} \\
\text { Potencia 350W }\end{array}$ \\
\hline & & Servidor IBM P520 & $\begin{array}{l}\text { Tipo: Físico } \\
\text { Procesador: IBM }\end{array}$ \\
\hline
\end{tabular}




\begin{tabular}{|c|c|c|c|}
\hline UBICACIÓN & AMBIENTES & SERVIDORES & DETALLE \\
\hline & & & $\begin{array}{l}\text { Aplicaciones: AIX } 520 \\
\text { Discos:1 } \\
\text { Capacidad: 600G } \\
\text { Potencia 350W }\end{array}$ \\
\hline & & Servidor OV Manager & $\begin{array}{l}\text { Tipo: Físico } \\
\text { Procesador: IBM } \\
\text { Aplicaciones: Oracle Linux } 5 \\
\text { Discos:1 } \\
\text { Capacidad: 1T } \\
\text { Potencia 350W }\end{array}$ \\
\hline & & Servidor Sharepoint & $\begin{array}{l}\text { Tipo: Físico } \\
\text { Procesador: Intel } \\
\text { Aplicaciones: Server } 2016 \\
\text { Discos:1 } \\
\text { Capacidad: 2T } \\
\text { Potencia 350W }\end{array}$ \\
\hline & & Servidor Asterisk & $\begin{array}{l}\text { Tipo: Físico } \\
\text { Procesador: IBM } \\
\text { Aplicaciones: Linux } 5 \\
\text { Discos: } 1 \\
\text { Capacidad: } 75 \mathrm{G} \\
\text { Potencia 350W }\end{array}$ \\
\hline & & $\begin{array}{l}\text { Servidor de Base de } \\
\text { Datos 10gr2 }\end{array}$ & $\begin{array}{l}\text { Tipo: Físico } \\
\text { Procesador: Intel } \\
\text { Aplicaciones: Linux } \\
\text { Discos:2 } \\
\text { Capacidad: 250G, 320G } \\
\text { Potencia 350W }\end{array}$ \\
\hline & & Servidor de Cámaras & $\begin{array}{l}\text { Tipo: Físico } \\
\text { Procesador: Intel } \\
\text { Aplicaciones: Windows } 7 \\
\text { Discos:1 } \\
\text { Capacidad: 1T } \\
\text { Potencia 350W }\end{array}$ \\
\hline & & Servidor Producción & $\begin{array}{l}\text { Tipo: Físico } \\
\text { Procesador: Supermicro } \\
\text { Aplicaciones: Oracle, Linux } \\
\text { Discos:3 } \\
\text { Capacidad: 500G, 1T, 1T } \\
\text { Potencia 350W }\end{array}$ \\
\hline & & Servidor Subversion & $\begin{array}{l}\text { Tipo: Físico } \\
\text { Procesador: Intel } \\
\text { Aplicaciones: Linux } \\
\text { Discos:1 } \\
\text { Capacidad: 1T } \\
\text { Potencia 350W }\end{array}$ \\
\hline & & Servidor Shared & Tipo: Físico \\
\hline
\end{tabular}




\begin{tabular}{|c|c|c|c|}
\hline UBICACIÓN & AMBIENTES & SERVIDORES & DETALLE \\
\hline & & & $\begin{array}{l}\text { Procesador: Supermicro } \\
\text { Aplicaciones: Oracle, Linux } \\
\text { Discos:3 } \\
\text { Capacidad: 1T, } 2 \mathrm{~T}, 2 \mathrm{~T} \\
\text { Potencia 350W }\end{array}$ \\
\hline & & $\begin{array}{c}\text { Servidor de Base de } \\
\text { Datos } 11 \mathrm{gr} 2\end{array}$ & $\begin{array}{l}\text { Tipo: Físico } \\
\text { Procesador: Intel } \\
\text { Aplicaciones: Linux } \\
\text { Discos:1 } \\
\text { Capacidad: } 500 \mathrm{G} \\
\text { Potencia } 350 \mathrm{~W}\end{array}$ \\
\hline & & $\begin{array}{c}\text { Servidor de Base de } \\
\text { Datos } 11 \mathrm{gr} 3\end{array}$ & $\begin{array}{l}\text { Tipo: Físico } \\
\text { Procesador: Intel } \\
\text { Aplicaciones: Linux } \\
\text { Discos:1 } \\
\text { Capacidad: } 1 \mathrm{~T} \\
\text { Potencia 350W }\end{array}$ \\
\hline & & Servidor Vware & $\begin{array}{l}\text { Tipo: Físico } \\
\text { Procesador: Intel } \\
\text { Aplicaciones: Linux } 5.5 \\
\text { Discos:1 } \\
\text { Capacidad: 2T } \\
\text { Potencia 350W }\end{array}$ \\
\hline & & Servidor JIRA & $\begin{array}{l}\text { Tipo: Físico } \\
\text { Procesador: Intel } \\
\text { Aplicaciones: Linux } 7.2 \\
\text { Discos:2 } \\
\text { Capacidad: 2T,2T } \\
\text { Potencia 350W }\end{array}$ \\
\hline & & $\begin{array}{c}\text { Servidor de base de } \\
\text { Datos MYSQL }\end{array}$ & $\begin{array}{l}\text { Tipo: Físico } \\
\text { Procesador: Intel } \\
\text { Aplicaciones: Linux } 7.2 \\
\text { Discos:2 } \\
\text { Capacidad: 1T,2T } \\
\text { Potencia 350W }\end{array}$ \\
\hline & Administración & & \\
\hline
\end{tabular}

Nota: Se ha descrito el tipo, procesador, aplicación discos, capacidad y potencia de cada servidor. Adaptado de “Manual de Configuración”, por El Objeto de Estudio, 2017. 


\section{(1)}

○ COMUNICACIONES FÍSICAS / ESPECIFICACIONES DE HARDWARE Y RED

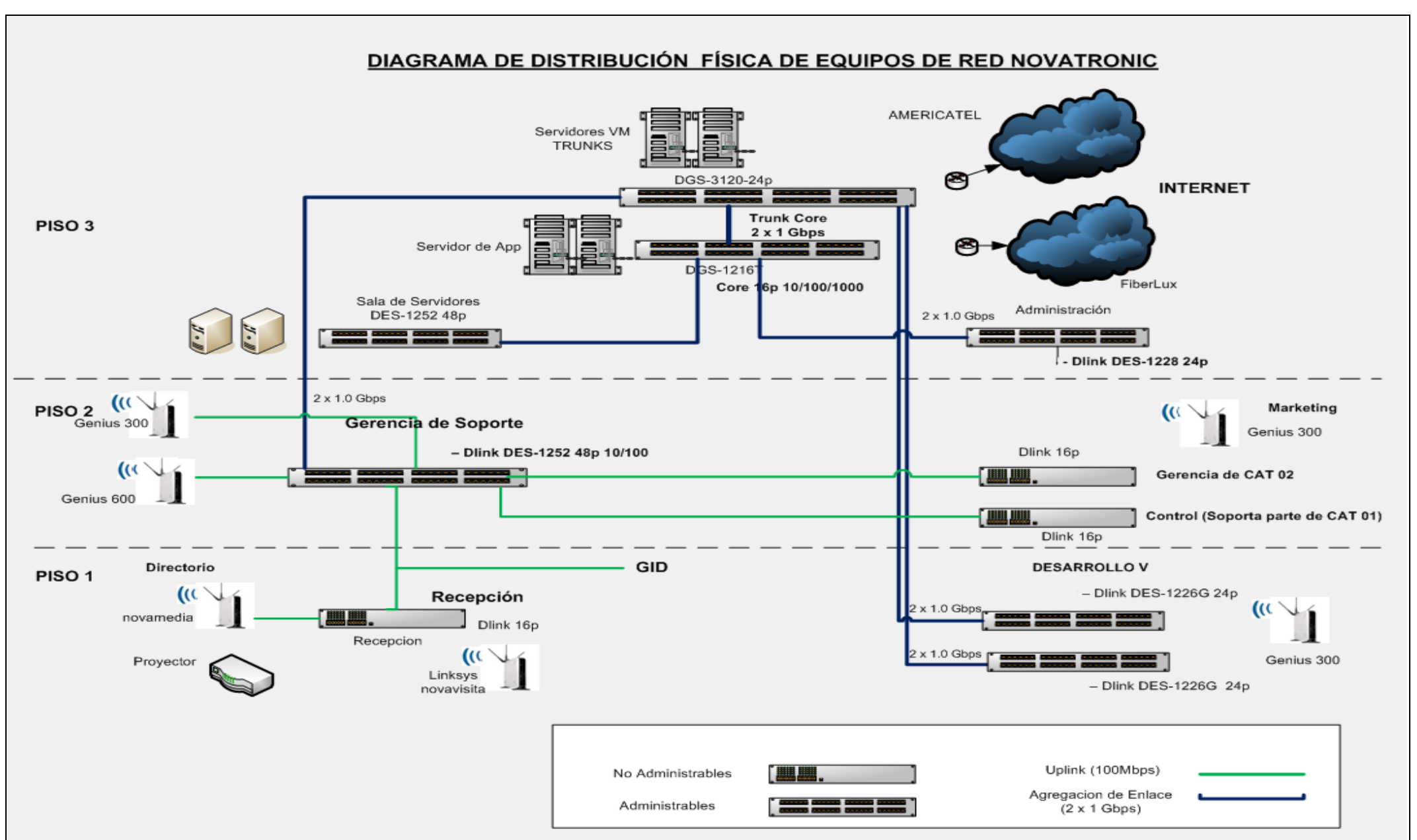

Figura 26. Diagrama de Distribución Física de Equipos de Red (TOBE). Adaptado de “Manual de Operación”, por El Objeto de Estudio, 2017. 


\section{$\boldsymbol{1}$}

\section{ANÁLISIS DE BRECHAS}

\section{Tabla 74}

Análisis de brechas - Arquitectura de Negocio del Primer Subproceso

\begin{tabular}{|c|c|c|c|c|c|c|}
\hline \multicolumn{7}{|c|}{ ARQUITECTURA DE NEGOCIO - SUBPROCESO DE CONTROL Y SEGUIMIENTO DE PROYECTOS (CSP) } \\
\hline \multirow{2}{*}{$\begin{array}{l}\text { Arquitectura línea base } \\
\qquad(\text { AS IS) }\end{array}$} & \multicolumn{6}{|c|}{ Arquitectura Objetivo (TO BE) } \\
\hline & $\begin{array}{c}\text { Revisar la última } \\
\text { actualización de la Bitácora } \\
\text { de Seguimiento de Proyectos. }\end{array}$ & $\begin{array}{l}\text { Monitorear las } \\
\text { actividades del } \\
\text { proyecto. }\end{array}$ & $\begin{array}{l}\text { Revisar el } \\
\text { estado del } \\
\text { proyecto. }\end{array}$ & $\begin{array}{l}\text { Gestionar el } \\
\text { proyecto. }\end{array}$ & $\begin{array}{l}\text { Actualizar la Bitácora } \\
\text { de seguimiento de } \\
\text { proyectos. }\end{array}$ & Acción \\
\hline $\begin{array}{l}\text { Revisar la última actualización } \\
\text { de la Bitácora de Seguimiento } \\
\text { de Proyectos. }\end{array}$ & $\mathbf{S}$ & & & & & \\
\hline $\begin{array}{l}\text { Monitorear las actividades del } \\
\text { proyecto. }\end{array}$ & & A & & & & \\
\hline Revisar el estado del proyecto. & & & $\mathbf{S}$ & & & \\
\hline Gestionar el proyecto. & & & & $\mathbf{S}$ & & \\
\hline $\begin{array}{l}\text { Actualizar la Bitácora de } \\
\text { seguimiento de proyectos. }\end{array}$ & & & & & $\mathbf{S}$ & \\
\hline Acción & & & & & & \\
\hline
\end{tabular}

Nota: Se ha descrito los actividades del proceso de ambas arquitecturas (ASIS y TOBE), con la cual se identificarán las brechas existentes (Leyenda: $\mathrm{S}=$ Se mantiene, A =

Actualizar, I = Implementar, E = Eliminar). Adaptado de "Procedimiento de Gestión de Proyecto", por El Objeto de Estudio, 2017. 
Tabla 75

Análisis de brechas - Arquitectura de Negocio del Segundo Subproceso

\begin{tabular}{|c|c|c|c|c|c|c|}
\hline \multicolumn{7}{|c|}{ ARQUITECTURA DE NEGOCIO - SUBPROCESO DE GESTIÓN DE PROYECTOS } \\
\hline \multirow{2}{*}{$\begin{array}{c}\text { Arquitectura línea base (AS } \\
\text { IS) }\end{array}$} & \multicolumn{6}{|c|}{ Arquitectura Objetivo (TO BE) } \\
\hline & $\begin{array}{l}\text { Analizar la información } \\
\text { obtenida durante la } \\
\text { reunión de seguimiento de } \\
\text { proyecto. }\end{array}$ & $\begin{array}{l}\text { Revisar si el proyecto } \\
\text { posee } \\
\text { correctivas acciones } \\
\text { de cierre. }\end{array}$ & $\begin{array}{l}\text { ¿Existen } \\
\text { problemas? }\end{array}$ & $\begin{array}{l}\text { Tomar } \\
\text { acciones } \\
\text { correctivas }\end{array}$ & $\begin{array}{l}\text { Debemos elaborar o } \\
\text { actualizar los } \\
\text { entregables de } \\
\text { gestión del proyecto }\end{array}$ & Acción \\
\hline $\begin{array}{l}\text { Analizar la información obtenida } \\
\text { durante la reunión de seguimiento } \\
\text { de proyecto. }\end{array}$ & $\mathbf{S}$ & & & & & \\
\hline $\begin{array}{l}\text { Revisar si el proyecto posee } \\
\text { acciones correctivas pendientes de } \\
\text { cierre. }\end{array}$ & & $\mathbf{S}$ & & & & \\
\hline ¿Existen problemas? & & & $\mathbf{S}$ & & & \\
\hline Tomar acciones correctivas & & & & $\mathbf{S}$ & & \\
\hline $\begin{array}{l}\text { Debemos elaborar o actualizar los } \\
\text { entregables de gestión del proyecto }\end{array}$ & & & & & A & \\
\hline Acción & & & & & & \\
\hline
\end{tabular}

Nota: Se ha descrito los actividades del proceso de ambas arquitecturas (ASIS y TOBE), con la cual se identificarán las brechas existentes (Leyenda: $\mathrm{S}=$ Se mantiene, A =

Actualizar, I = Implementar, E = Eliminar). Adaptado de "Procedimiento de Gestión de Proyecto", por El Objeto de Estudio, 2017. 


\section{(1)}

Las brechas encontradas en la arquitectura de negocios para el proceso de Gestión de Proyectos son las siguientes:

BN01: Se deberá actualizar el procedimiento con respecto a la tarea de monitorear las actividades del proyecto, ya que desde la solución propuesta (Sistema JIRA) se podrá monitorear dichas actividades (Matriz de Trazabilidad, Cronograma del Proyecto, Riesgos del Proyectos y Bitácora de Seguimiento).

BN02: Se deberá actualizar el procedimiento con respecto a la tarea debemos elaborar o actualizar los entregables de gestión del proyecto, ya que la solución propuesta (Sistema JIRA) se podrá elaborar o actualizar dichas actividades (Matriz de Trazabilidad, Cronograma del Proyecto, Riesgos del Proyectos y Bitácora de Seguimiento). 


\section{(}

Tabla 76

Análisis de brechas de la Arquitectura de Aplicación

\begin{tabular}{|c|c|c|}
\hline \multicolumn{2}{|c|}{ ARQUITECTURA DE APLICACIÓN } \\
\hline \multirow{2}{*}{ Arquitectura línea base (AS IS) } & Arquitectura Objetivo (TO BE) \\
\cline { 2 - 3 } & JIRA & Acción \\
\hline IMPORT/EXPORT & & E \\
\hline SARA & & E \\
\hline Procesador de Cronogramas & & E \\
\hline Acción & I \\
\hline
\end{tabular}

Nota: Se ha descrito las aplicaciones de ambas arquitecturas (ASIS y TOBE), con la cual se identificarán las brechas existentes (Leyenda: $\mathrm{S}=$ Se mantiene, $\mathrm{A}=$ Actualizar, $\mathrm{I}=$ Implementar, $\mathrm{E}=$ Eliminar). Adaptado de “Manual de Configuración”, por El Objeto de Estudio, 2017.

Las brechas encontradas en la arquitectura de aplicación para el proceso de Gestión de Proyectos son las siguientes:

BA01: Los sistemas IMPORT/EXPORT, SARA y Procesador de Cronogramas serán eliminados ya que estas 3 aplicaciones serán sustituidas por el nuevo sistema JIRA.

BA02: El sistema JIRA será una nueva aplicación a implementar el cual sustituirá los 3 sistemas existentes para la gestión de proyectos. 
Tabla 77

Análisis de brechas de la Arquitectura Tecnológica

\begin{tabular}{|c|c|c|c|c|c|c|c|}
\hline \multicolumn{8}{|c|}{ ARQUITECTURA TECNOLOGÍCA } \\
\hline \multirow{2}{*}{$\begin{array}{l}\text { Arquitectura línea } \\
\text { base (AS IS) }\end{array}$} & \multicolumn{7}{|c|}{ Arquitectura Objetivo (TO BE) } \\
\hline & $\begin{array}{c}14 \text { Switch } \\
\text { Dlink }\end{array}$ & 1 Proyector & Servidores & Servidor VM TRUNKS & $\begin{array}{l}\text { Servidor de } \\
\text { App }\end{array}$ & Router Fiberlux & $\begin{array}{c}\text { Router } \\
\text { Americatel }\end{array}$ \\
\hline 14 Switch Dlink & $\mathbf{S}$ & & & & & & \\
\hline 1 Proyector & & $\mathbf{S}$ & & & & & \\
\hline Servidores & & & I & & & & \\
\hline Servidor VM TRUNKS & & & & $\mathbf{S}$ & & & \\
\hline Servidor de App & & & & & $\mathbf{S}$ & & \\
\hline Router Fiberlux & & & & & & $\mathbf{S}$ & \\
\hline Router Americatel & & & & & & & $\mathbf{S}$ \\
\hline
\end{tabular}

Nota: Se ha descrito los componentes de ambas arquitecturas (ASIS y TOBE), con la cual se identificarán las brechas existentes (Leyenda: $\mathrm{S}=$ Se mantiene, A = Actualizar, I

= Implementar, E = Eliminar). Adaptado de "Manual de Configuración”, por El Objeto de Estudio, 2017.

La brecha encontrada en la arquitectura tecnológica para el proceso de Gestión de Proyectos son las siguientes:

BT01:

La sala de servidores deberá implementar 2 nuevos equipos para que soporte la nueva aplicación y su base de datos. 


\section{$\boldsymbol{( 1 )}$}

Tabla 78

Análisis de brechas de la Arquitectura de Datos

\section{ARQUITECTURA DE DATOS}

\begin{tabular}{|c|c|c|c|c|c|c|c|c|c|c|c|c|c|c|c|c|c|c|c|c|c|c|c|c|c|}
\hline Arquitectura & \multicolumn{25}{|c|}{ Arquitectura Objetivo (TO BE) } \\
\hline línea base & TD & $\mathrm{TD}$ & $\mathrm{TD}$ & $\mathrm{TD}$ & TD & TD & TD & TD & TD & TD & TD & TD & $\mathrm{TD}$ & TD & TD & $\mathrm{TD}$ & TD & TD & TD & TD & TD & TD & TD & TD & Acción \\
\hline (AS IS) & 01 & 02 & 03 & 04 & 05 & 06 & 07 & 08 & 09 & 10 & 11 & 12 & 13 & 14 & 15 & 16 & 17 & 18 & 19 & 20 & 21 & 22 & 23 & 24 & \\
\hline TLB01 & & & & & & & & & & & & & & & & & & & & & & & & & $\mathbf{E}$ \\
\hline TLB02 & & & & & & & & & & & & & & & & & & & & & & & & & $\mathbf{E}$ \\
\hline TLB03 & & & & & & & & & & & & & & & & & & & & & & & & & $\mathbf{E}$ \\
\hline TLB04 & & & & & & & & & & & & & & & & & & & & & & & & & $\mathbf{E}$ \\
\hline TLB05 & & & & & & & & & & & & & & & & & & & & & & & & & $\mathbf{E}$ \\
\hline TLB06 & & & & & & & & & & & & & & & & & & & & & & & & & $\mathbf{E}$ \\
\hline TLB07 & & & & & & & & & & & & & & & & & & & & & & & & & $\mathbf{E}$ \\
\hline TLB08 & & & & & & & & & & & & & & & & & & & & & & & & & $\mathbf{E}$ \\
\hline TLB09 & & & & & & & & & & & & & & & & & & & & & & & & & $\mathbf{E}$ \\
\hline TLB10 & & & & & & & & & & & & & & & & & & & & & & & & & $\mathbf{E}$ \\
\hline TLB11 & & & & & & & & & & & & & & & & & & & & & & & & & $\mathbf{E}$ \\
\hline TLB12 & & & & & & & & & & & & & & & & & & & & & & & & & $\mathbf{E}$ \\
\hline TLB13 & & & & & & & & & & & & & & & & & & & & & & & & & $\mathbf{E}$ \\
\hline
\end{tabular}




\section{(}

\begin{tabular}{|c|c|c|c|c|c|c|c|c|c|c|c|c|c|c|c|c|c|c|c|c|c|c|c|c|c|}
\hline TLB14 & & & & & & & & & & & & & & & & & & & & & & & & & $\mathbf{E}$ \\
\hline TLB15 & & & & & & & & & & & & & & & & & & & & & & & & & $\mathbf{E}$ \\
\hline TLB16 & & & & & & & & & & & & & & & & & & & & & & & & & $\mathbf{E}$ \\
\hline TLB17 & & & & & & & & & & & & & & & & & & & & & & & & & $\mathbf{E}$ \\
\hline TLB18 & & & & & & & & & & & & & & & & & & & & & & & & & $\mathbf{E}$ \\
\hline TLB19 & & & & & & & & & & & & & & & & & & & & & & & & & $\mathbf{E}$ \\
\hline TLB20 & & & & & & & & & & & & & & & & & & & & & & & & & $\mathbf{E}$ \\
\hline TLB21 & & & & & & & & & & & & & & & & & & & & & & & & & $\mathbf{E}$ \\
\hline TLB22 & & & & & & & & & & & & & & & & & & & & & & & & & $\mathbf{E}$ \\
\hline TLB23 & & & & & & & & & & & & & & & & & & & & & & & & & $\mathbf{E}$ \\
\hline TLB24 & & & & & & & & & & & & & & & & & & & & & & & & & $\mathbf{E}$ \\
\hline Acción & I & I & I & I & I & I & I & I & I & I & I & I & $\mathbf{I}$ & I & I & I & I & I & I & $\mathbf{I}$ & I & I & $\mathbf{I}$ & I & \\
\hline
\end{tabular}

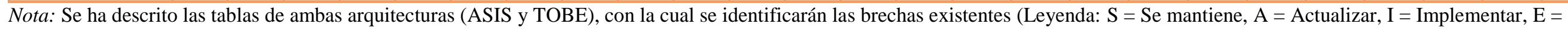
Eliminar). Adaptado de "Manual de Configuración”, por El Objeto de Estudio, 2017. 


\section{(}

\section{Tablas Línea Base}

Tabla 79

Nomenclatura tablas línea base

\begin{tabular}{|c|c|}
\hline Código de Tabla & Nombre de Tabla \\
\hline TLB01 & tsetapaproyecto \\
\hline TLB02 & tpcliente \\
\hline TLB03 & tsproccrongavanproyetapa \\
\hline TLB04 & tdproyectoetapaproyecto \\
\hline TLB05 & tpaccion \\
\hline TLB06 & tslineabaseproyectoetapa \\
\hline TLB07 & tslineabaseproyectohito \\
\hline TLB08 & tslineabaseproyectotarea \\
\hline TLB09 & tsarea \\
\hline TLB 10 & tslineabaseproyecto \\
\hline TLB 11 & tsproducto \\
\hline TLB 12 & tptarea \\
\hline TLB 13 & taequipotrabajo \\
\hline TLB 14 & estadoproyectos \\
\hline TLB 15 & tdproyectoproducto \\
\hline TLB16 & Tpproyecto \\
\hline TLB 17 & tatablatablas \\
\hline TLB 18 & tacabeceratablatablas \\
\hline TLB 19 & tsproccrongavanproyrecurso \\
\hline TLB20 & tsproccrongavanproy \\
\hline TLB21 & tsproccrongavanproyhito \\
\hline TLB22 & tssolicitudcambio \\
\hline TLB23 & tspersonal \\
\hline
\end{tabular}

Nota: Se ha descrito el código y nombre de cada tabla de la arquitectura línea base. Adaptado de "Manual de Configuración”, por El Objeto de Estudio, 2017. 


\section{(}

\section{Tablas Destino}

Tabla 80

Nomenclatura tablas línea destino

\begin{tabular}{|c|c|}
\hline Código de Tabla & Nombre de Tabla \\
\hline TD01 & ao_589059_rule_config \\
\hline TD02 & ao_589059_rule_cfg_component \\
\hline TD03 & ao_563aee_object_entity \\
\hline TD04 & ao_563aee_media_link_entity \\
\hline TD05 & ao_563aee_target_entity \\
\hline TD06 & ao_563aee_actor_entity \\
\hline TD07 & ao_563aee_activity_entity \\
\hline TD08 & ao_589059_audit_item \\
\hline TD09 & ao_589059_automation_queue \\
\hline TD10 & jiraaction \\
\hline TD11 & jiraworkflows \\
\hline TD12 & jiraissue \\
\hline TD13 & jiraworkflowstatuses \\
\hline TD14 & jiraperms \\
\hline TD15 & workflowscheme \\
\hline TD16 & worklog \\
\hline TD17 & workflowschemeentity \\
\hline TD18 & projectroleactor \\
\hline TD19 & projectversion \\
\hline TD20 & projectchangedtime \\
\hline TD21 & projectrole \\
\hline TD22 & project_key \\
\hline TD23 & project \\
\hline TD24 & projectcategory \\
\hline
\end{tabular}

Nota: Se ha descrito el código y nombre de cada tabla de la arquitectura línea destino. Elaboración propia. 


\section{$(1)$}

Las brechas encontradas en la arquitectura de datos para el proceso de Gestión de Proyectos son las siguientes:

BD01: Las tablas de la línea base serán eliminadas debido a que como son parte de las antiguas aplicaciones estas serán sustituidos por las nuevas tablas de la nueva aplicación.

BD02: Las tablas del destino serán nuevas tablas a implementar debido a que la solución del nuevo aplicativo usa un nuevo modelo de datos. 


\section{(1)}

\section{OPORTUNIDADES Y SOLUCIONES}

Se tendrá que desarrollar el siguiente entregable:

- Plan de Implementación y Migración (Implementation and Migration Plan), el cual contiene:

\section{CUADRO RESUMEN DE PLAN DE MIGRACIÓN}

Tabla 81

Cuadro resumen de Plan de Migración

\begin{tabular}{|c|c|c|c|c|c|}
\hline Brecha & Proyecto & Problema & $\operatorname{Costos}(\mathrm{S} /)$. & Solución Potencial & Riesgos \\
\hline BN01 & $\begin{array}{l}\text { Mejora del } \\
\text { procedimiento } \\
\text { de Gestión de } \\
\text { Proyectos }\end{array}$ & $\begin{array}{l}\text { El procedimiento de Gestión de } \\
\text { Proyectos no se encuentra } \\
\text { actualizado con el nuevo } \\
\text { sistema que se implementará, } \\
\text { ni con los entregables que se } \\
\text { podrán manejar desde dicho } \\
\text { sistema. }\end{array}$ & $5,000.00$ & $\begin{array}{l}\text { Se deberá actualizar el procedimiento } \\
\text { con respecto a la tarea de monitorear las } \\
\text { actividades del proyecto, ya que desde } \\
\text { la solución propuesta (Sistema JIRA) se } \\
\text { podrá monitorear dichas actividades } \\
\text { (Matriz de Trazabilidad, Cronograma } \\
\text { del Proyecto, Riesgos del Proyectos y } \\
\text { Bitácora de Seguimiento). }\end{array}$ & $\begin{array}{l}\text { - La no actualización } \\
\text { del procedimiento de } \\
\text { Gestión de Proyectos } \\
\text { puede generar no } \\
\text { conformidades al } \\
\text { proceso. }\end{array}$ \\
\hline BN02 & & & & $\begin{array}{l}\text { Se deberá actualizar el procedimiento } \\
\text { con respecto a la tarea Debemos }\end{array}$ & \\
\hline
\end{tabular}




\begin{tabular}{|c|c|c|c|c|c|}
\hline Brecha & Proyecto & Problema & Costos (S/.) & Solución Potencial & Riesgos \\
\hline & & & & $\begin{array}{l}\text { elaborar o actualizar los entregables de } \\
\text { gestión del proyecto, ya que la solución } \\
\text { propuesta del sistema JIRA se podrá } \\
\text { elaborar o actualizar dichas actividades } \\
\text { (Matriz de Trazabilidad, Cronograma } \\
\text { del Proyecto, Riesgos del Proyectos y } \\
\text { Bitácora de Seguimiento). }\end{array}$ & \\
\hline $\begin{array}{l}\text { BA01 } \\
\text { BA02 }\end{array}$ & $\begin{array}{l}\text { Implementación } \\
\text { Software JIRA } \\
7.4\end{array}$ & $\begin{array}{l}\text { Los subsistemas que usa la } \\
\text { gestión de proyectos se } \\
\text { encuentran aislados, en cada } \\
\text { subsistema se debe realizar } \\
\text { ciertas actividades para poder } \\
\text { alimentar la información y } \\
\text { tener un cronograma } \\
\text { actualizado. }\end{array}$ & $55,000.00$ & $\begin{array}{l}\text { Se implementará un nuevo sistema que } \\
\text { ahora reemplazará los } 3 \text { subsistemas } \\
\text { existentes para contar con una mejor } \\
\text { integración de la información en un solo } \\
\text { aplicativo. }\end{array}$ & $\begin{array}{l}\text { - No se tenga el } \\
\text { soporte debido para la } \\
\text { aplicación. } \\
\text { - El sistema no } \\
\text { cumpla con las } \\
\text { expectativas para } \\
\text { resolver el problema. }\end{array}$ \\
\hline BT01 & $\begin{array}{l}\text { Implementación } \\
\text { Software JIRA } \\
7.4\end{array}$ & $\begin{array}{l}\text { No se tiene } r \text { servidores } \\
\text { disponibles } \quad \text { para nuevas }\end{array}$ & $120,000.00$ & $\begin{array}{l}\text { Se procederá a realizar la compra de } 2 \\
\text { nuevos servidores para el alojamiento }\end{array}$ & $\begin{array}{l}\text { - Que los servidores } \\
\text { no sean aptos para las }\end{array}$ \\
\hline
\end{tabular}




\section{(1)}

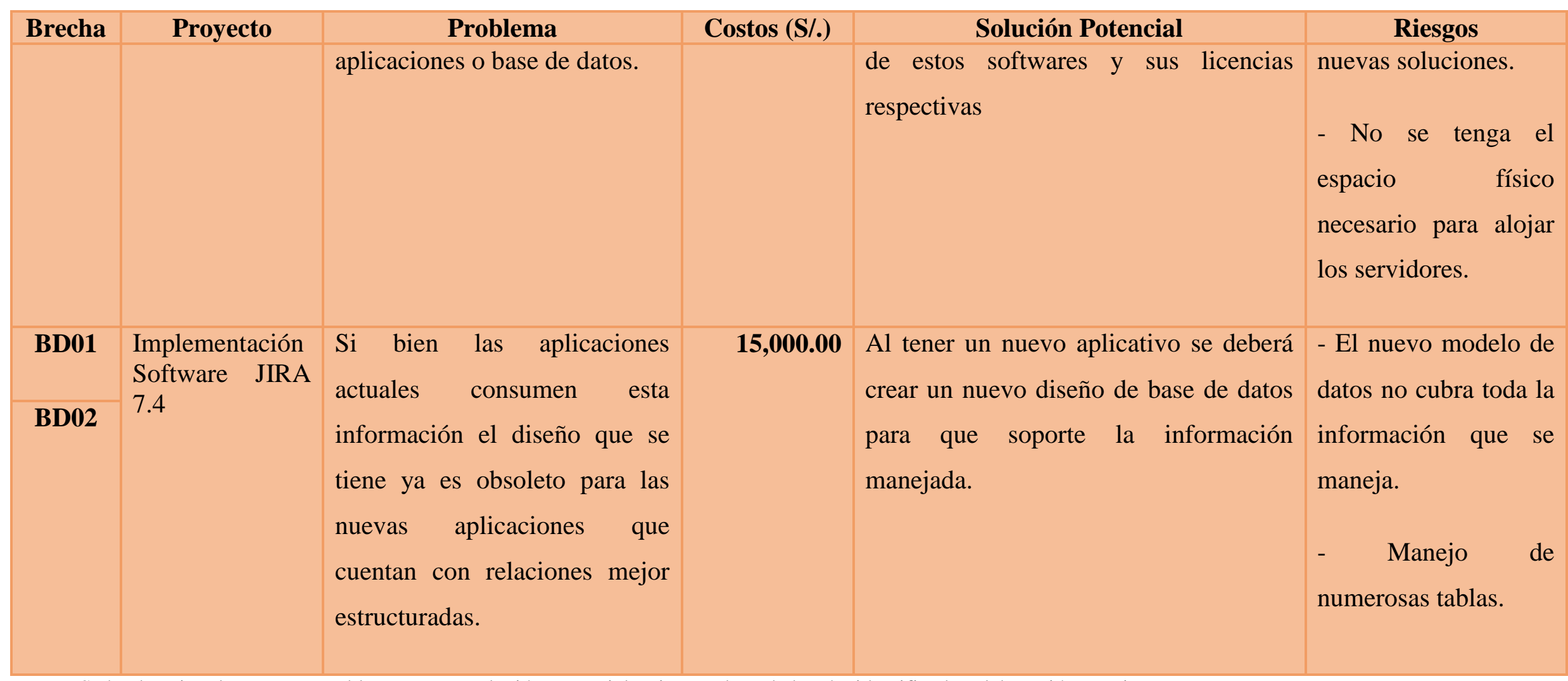

Nota: Se ha descrito el proyecto, problema, costo, solución potencial y riesgos de cada brecha identificada. Elaboración propia. 


\section{(1)}

ESTRATEGIA DE IMPLEMENTACIÓN Y MIGRACIÓN

- ENFOQUE DE LA SECUENCIA DE LA IMPLEMENTACIÓN

Tabla 82

Cuadro del Enfoque de secuencia de Implementación

\begin{tabular}{|c|c|c|}
\hline $\begin{array}{c}\text { Proyecto } \\
\text { Mejora del procedimiento }\end{array}$ & Prioridad & \multicolumn{1}{|c|}{ Motivo } \\
\hline de Gestión de Proyectos & 1 & Se realizará en paralelo con el proyecto \\
Implementación Software JIRA 7.4, ya que \\
todo cambio en la organización debe estar \\
documentado debido a que la empresa cuenta \\
con certificaciones de calidad.
\end{tabular}

Nota: Se ha descrito la prioridad y el motivo de los proyectos a implementar. Elaboración propia. 
DESGLOSE DE LA IMPLEMENTACIÓN DE PROYECTOS Y CARTERAS

- ESTRUCTURA DE DESGLOSE DEL TRABAJO

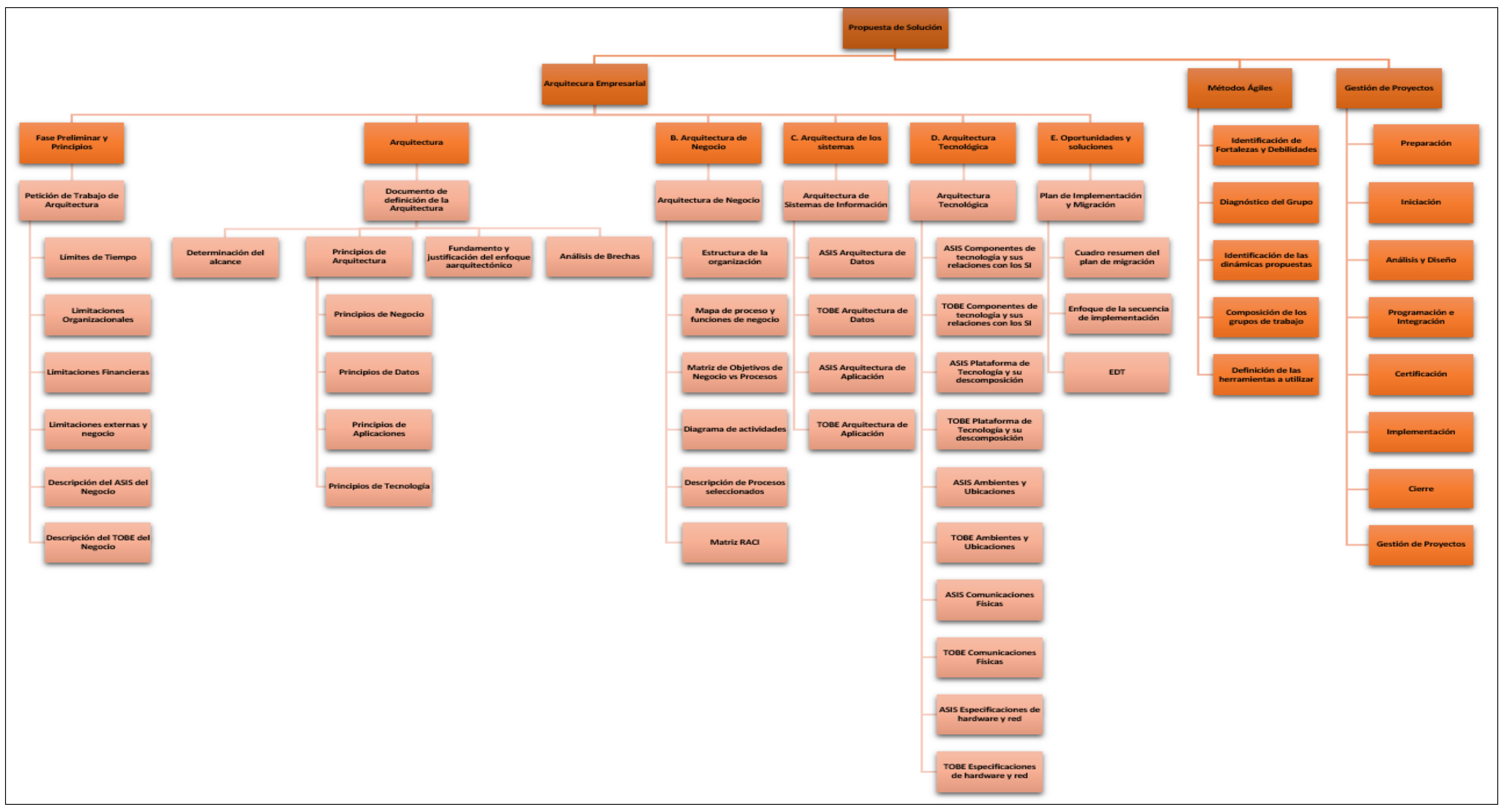

Figura 27. Estructura de desglose de trabajo. Adaptado de “Welcome to the TOGAF® Standard, Version 9.2, a standard of The Open Group”, por The Open Group, 1999 - 2018 


\section{(1)}

\section{MÉTODOS ÁGILES PARA EL DESARROLLO DE SOFTWARE}

\section{IDENTIFICACIÓN DE FORTALEZAS Y DEBILIDADES}

Como parte de la explicación de la identificación de las fortalezas y debilidades, primero se detallará un breve concepto sobre el análisis FODA.

\section{Análisis FODA:}

Según lo indicado por Cabrera (2018), es un análisis de los puntos FUERTES y DÉBILES de la organización, en relación a las OPORTUNIDADES y AMENAZAS del entorno.

Recoge:

- los puntos Fuertes en que nos debemos apoyar

- los puntos Débiles que debemos superar

- las Oportunidades que tenemos que aprovechar

- las Amenazas de las que nos tenemos que defender

Adicionalmente, como parte final de esta identificación se usará el marco Cynefin por lo que se menciona de igual forma un breve concepto de este.

\section{Cynefin:}

Según lo indicado por Subauste (2018), en él se representan las cinco situaciones en las que una organización puede encontrarse, y es cada una de esas situaciones las que determinarán la manera en que deberíamos actuar. Se detalla cada una de ellas:

\section{- Obvio (Simple): Mejores prácticas}

○ Todos conocen cómo hacer las cosas.

- Existen las mejores prácticas y son conocidas.

- Serie lógica de pasos y procesos repetitivos. 


\section{(1)}

- La relación causa - efecto es obvia para todos.

- Cualquiera puede hacerlo conociendo los pasos. Es obvio.

- Procesos compuestos por pasos bien definidos mejor que Scrum

- Complicado: Buenas prácticas

○ Problemas complicados.

○ Hay buenas prácticas.

- Se requiere de expertos.

○ Múltiples soluciones correctas.

○ Se busca eficiencia y eficacia más que soluciones nuevas.

- Complejo: Prácticas emergentes

○ Resultados impredecibles.

○ No hay mejores ni buenas prácticas.

- No sabemos si la solución va a funcionar.

- Soluciones poco replicables.

○ Se requiere creatividad e innovación.

- El conocimiento emerge durante el trabajo.

○ Incertidumbre.

- Caótico: Práctica novedosas

○ Emergencia.

- Se da prioridad a la velocidad de solución antes que a la calidad.

- Existe una crisis, buscamos orden.

○ Improvisación.

- Desordenado: Práctica novedosas

○ Total vacío.

- Incapacidad de determinar qué hacer.

○ Se aplican soluciones equivocadas.

○ Se repite el error.

○ Los resultados son negativos pero no tienen explicación.

- Nos basamos solamente en la forma de hacer y no en los resultados. 


\section{(1)}

A continuación, se detalla en el siguiente recuadro las fortalezas, oportunidades, amenazas y debilidades del Objeto de Estudio.

\section{Tabla 83}

Cuadro del Análisis FODA del Objeto de Estudio

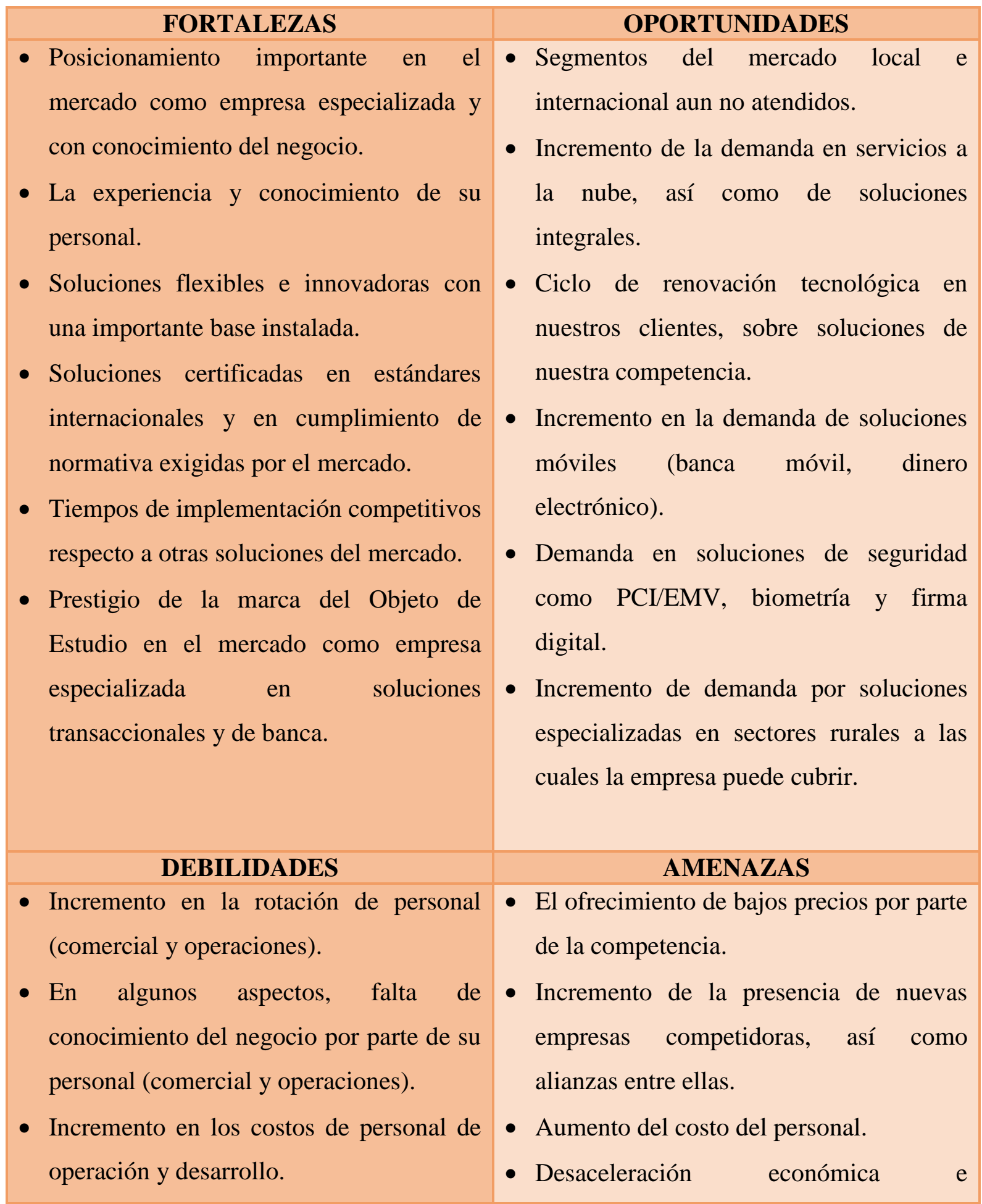




\section{(1)}

- Falta de estandarización de procesos en el incertidumbre política. área comercial.

- Cantidad de productos legacy que implica tiempos de mantenimiento por el área operativa.

- No se tiene mapeado por el área comercial la venta realizada por líneas de productos.

Nota: Se ha descrito las fortalezas, oportunidades debilidades y amenazas del Objeto de Estudio. Adaptado de “Manual de Calidad”, por El Objeto de Estudio, 2017

Seguidamente, se realiza el cuadro de fortalezas y debilidades del grupo de trabajo que se hará cargo del proyecto.

Tabla 84

Cuadro del Análisis de Fortalezas y Debilidades del grupo de trabajo

\begin{tabular}{|c|c|}
\hline FORTALEZAS & DEBILIDADES \\
\hline $\begin{array}{l}\text { - Amplio conocimiento técnico en } \\
\text { lenguaje de programación JAVA } \\
\text { - Capacitaciones periódicas sobre nuevas } \\
\text { tecnologías. } \\
\text { - Conocimiento compartido sobre el flujo } \\
\text { de los procesos internos. } \\
\text { - Apoyo mutuo en el grupo de mismos } \\
\text { roles. } \\
\text { - Desempeño individual adecuado. } \\
\text { - Conocimiento sobre Scrum. }\end{array}$ & $\begin{array}{l}\text { - Poca comunicación entre los diferentes } \\
\text { roles del equipo de trabajo. } \\
\text { - Rotación continua del personal de } \\
\text { operaciones por demandas del mercado } \\
\text { laboral. } \\
\text { - Malas planificaciones por parte del líder } \\
\text { técnico. } \\
\text { - Desmotivación por carga laboral extra por } \\
\text { proyectos exigentes. } \\
\text { Falta de conocimiento funcional sobre } \\
\text { nuevos proyectos de otros sectores. }\end{array}$ \\
\hline
\end{tabular}

Nota: Se ha descrito las fortalezas y debilidades del grupo de trabajo. Adaptado de "Manual de funciones y perfiles de puestos", por El Objeto de Estudio, 2017. 


\section{(1)}

El proyecto a realizar será la actualización de las tres herramientas que se usan actualmente para la gestión de proyectos por el software JIRA.

Después de haber realizado el análisis de las fortalezas y debilidades del grupo se procede a emplear el marco Cynefin para poder determinar en qué dominio se ubica actualmente.

Si bien el objeto de estudio es una consultora de software que tiene un equipo dedicado a la elaboración y gestión de proyectos no cuenta con las herramientas adecuadas para poder gestionar de manera eficiente los tiempos de cada proyecto, por ello se ha optado por implementar una nueva herramienta la cual ha tenido éxito en diversas empresas para la gestión de proyectos, seguimiento de incidencias, entre otros. Sabiendo que existen consultoras expertas dedicadas a la implementación de este producto se puede concluir que el objeto de estudio se encuentra en un dominio complicado en el cuadro de Cynefin.

\section{DIAGNÓSTICO DEL GRUPO}

Si bien el objeto de estudio está familiarizado en trabajar con metodología RUP, la Gerencia de Investigación, Desarrollo e Innovación ha realizado un análisis por cliente en función al rubro y la magnitud del proyecto, debido a ello han identificado que existe un gran porcentaje de clientes que demandan avances de funcionalidades tempranas, es decir visibilidad del software para que puedan validar que se está haciendo realmente lo solicitado.

Aparte de ello, se debe tener en cuenta que la empresa cuenta con CMMI (nivel 3) e ISO 9001:2008, donde CMMI garantiza calidad en sus procesos de desarrollo de software y el certificado ISO asegura la calidad de nuestros productos y procesos.

Como parte del diagnóstico del grupo, se procede a realizar un cuadro en base a las debilidades presentadas anteriormente y los posibles problemas que se pueden presentar en el equipo. 


\section{(1)}

Tabla 85

Cuadro del Análisis de Debilidades y Posibles Problemas

\begin{tabular}{|l|l|}
\hline \multicolumn{1}{|c|}{ DEBILIDADES } & \multicolumn{1}{|c|}{ POSIBLES PROBLEMAS } \\
\hline $\begin{array}{l}\text { Rotación continua del personal } \\
\text { de operaciones por demandas del } \\
\text { mercado laboral. }\end{array}$ & $\begin{array}{l}\text { Puede generar demoras en la elaboración de algún } \\
\text { proyecto en curso perjudicando el avance. Además, } \\
\text { cuando se tenga un nuevo recurso será necesario } \\
\text { entrenarlo a nivel técnico como funcional lo cual } \\
\text { demanda un tiempo. }\end{array}$ \\
\hline $\begin{array}{l}\text { Falta de conocimiento funcional } \\
\text { sobre nuevos proyectos de otros } \\
\text { sectores. }\end{array}$ & $\begin{array}{l}\text { Si no se tiene un equipo con los conocimientos } \\
\text { adecuados del ámbito del negocio puede generar } \\
\text { retrasos y baja calidad en el resultado final del proyecto. }\end{array}$ \\
\hline $\begin{array}{l}\text { Desmotivación por carga laboral } \\
\text { extra por proyectos exigentes }\end{array}$ & $\begin{array}{l}\text { El equipo se puede sentir estresado ocasionando un bajo } \\
\text { rendimiento el cual puede generar demoras en sus } \\
\text { actividades. }\end{array}$ \\
\hline $\begin{array}{l}\text { Poca comunicación entre los } \\
\text { diferentes roles del equipo de } \\
\text { trabajo }\end{array}$ & $\begin{array}{l}\text { Puede que la gestión del proyecto (documentos de } \\
\text { especificaciones, cambios, mejoras, etc.) no se realicen } \\
\text { de manera correcta ya que no existe una buena } \\
\text { comunicación entre los diferentes roles del proyecto. }\end{array}$ \\
\hline $\begin{array}{l}\text { Malas planificaciones por parte } \\
\text { del líder técnico }\end{array}$ & $\begin{array}{l}\text { Cambio de fechas pactadas, ajuste de tiempos } \\
\text { exagerados, reducción de alcance y retraso de inicio de } \\
\text { nuevos proyectos y todo esto debido a la mala } \\
\text { estimación. }\end{array}$ \\
\hline
\end{tabular}

Nota: Se ha descrito las debilidades y posibles problemas del grupo de trabajo. Elaboración Propia.

Después de haber realizado el diagnóstico del grupo se procederá a ejecutar este proyecto haciendo uso de Scrum, con el fin de ejecutar este framework en primera instancia en proyectos internos para luego poder replicarlo en proyectos externos. Sin embargo, se tendrá que elaborar los documentos mínimos que demanda el Objeto de estudio ya que cuenta con CMMI (nivel 3) e ISO 9001:2008. 


\section{(1)}

\section{IDENTIFICACIÓN DE LAS DINÁMICAS PROPUESTAS}

Habiendo realizado el diagnóstico del grupo y decidido que se empleará Scrum, se procede a realizar un breve concepto de las dinámicas que se adoptaran en el grupo de trabajo, el modo en cómo se ejecutarán y de qué forma beneficiaran al grupo.

\section{Sprint:}

Tal como indican Navarro, Fernández \& Morales (2013), es una ventana de tiempo donde se crea una versión utilizable del producto (incremento). Cada Sprint, es considerado como un proyecto independiente. Su duración máxima es de un mes. Un Sprint se compone de los siguientes elementos: reunión de planeación del Sprint, Daily Scrum, trabajo de desarrollo, revisión del Sprint y retrospectiva del Sprint.

\section{Sprint Planning:}

Se define su plan de trabajo: qué se va a entregar y cómo se logrará. Es decir, el diseño del sistema y la estimación de cantidad de trabajo. Esta actividad dura ocho horas para un Sprint de un mes. Si el Sprint tiene una duración menor, se asigna el tiempo de manera proporcional.

Tabla 86

Cuadro de Ejecución y beneficios del Sprint Planning

\begin{tabular}{|c|c|}
\hline MODO DE EJECUCIÓN & BENEFICIOS \\
\hline $\begin{array}{l}\text { - Cada lunes se realizará la planificación } \\
\text { de cada sprint. } \\
\text { - Se tomará una hora para realizar la } \\
\text { definición. } \\
\text { - Todo el grupo de trabajo elaborará sus } \\
\text { acuerdos y compromisos. } \\
\text { - Todo el grupo de trabajo deberá estar } \\
\text { presente en la planificación del sprint. }\end{array}$ & $\begin{array}{l}\text { - Se tendrá planificado las actividades a } \\
\text { realizar durante las } 2 \text { semanas que dura } \\
\text { cada sprint. } \\
\text { - Se tendrá definido los product backlog } \\
\text { ítems a ejecutar por el grupo de trabajo } \\
\text { en cada sprint. } \\
\text { - Se tendrá las metas a trazar en el sprint. }\end{array}$ \\
\hline
\end{tabular}

Nota: Se ha descrito el modo de ejecución y beneficios del Sprint Planning. Elaboración Propia. 


\section{4}

\section{Daily Scrum:}

Tal como indican Navarro, Fernández \& Morales (2013), es un evento del equipo de desarrollo de quince minutos, que se realiza cada día con el fin de explicar lo que se ha alcanzado desde la última reunión; lo que se hará antes de la siguiente; y los obstáculos que se han presentado. Este evento se desarrolla mediante una reunión que normalmente es sostenida de pie con los participantes reunidos formando un círculo, esto, para evitar que la discusión se extienda.

\section{Tabla 87}

Cuadro de Ejecución y beneficios del Daily Scrum

\section{MODO DE EJECUCIÓN}

\section{BENEFICIOS}

- El equipo de desarrollo tendrá su daily scrum a

- Permitirá que el equipo tenga una partir de las 9 y 30 am y como tiempo estimado mejor fluidez en la un total de 15 minutos. comunicación.

- El equipo de calidad tendrá su daily scrum a - Conocer posibles problemas partir de las 9 y 30 am y como tiempo estimado durante la ejecución de las un total de 15 minutos. actividades.

- Se realizará de manera presencial ya que el - Aclarar dudas que se presenten proyecto se hará in house. entre ambos equipos.

- Conocer los avances realizados después de cada jornada de trabajo.

Nota: Se ha descrito el modo de ejecución y beneficios del Daily Scrum. Elaboración Propia.

\section{Sprint Review:}

Tal como indican Navarro, Fernández \& Morales (2013), ocurre al final del Sprint y su duración es de cuatro horas para un proyecto de un mes (o una proporción de ese tiempo si la duración es menor). En esta etapa: el dueño del proyecto revisa lo que se hizo, identifica lo que no se hizo y discute acerca del Product Backlog; el equipo de desarrollo cuenta los problemas que encontró y la manera en que fueron resueltos, y muestra el producto y su funcionamiento. Esta reunión es de gran importancia para los siguientes Sprints. 


\section{$(\varphi$}

Tabla 88

Cuadro de Ejecución y beneficios del Sprint Review

\begin{tabular}{|c|c|}
\hline MODO DE EJECUCIÓN & BENEFICIOS \\
\hline $\begin{array}{l}\text { - Se tomará una hora para realizar la } \\
\text { revisión del sprint. } \\
\text { - Todo el grupo de trabajo deberá } \\
\text { estar presente en la revisión del } \\
\text { sprint. }\end{array}$ & $\begin{array}{l}\text { - Permitirá detectar problemas que se } \\
\text { presentaron en el anterior sprint } \\
\text { - Inventariar las actividades no realizadas en el } \\
\text { tiempo estimado. } \\
\text { - Aclarar dudas sobre requisitos no entendidos. } \\
\text { - Aceptación del producto owner de las tareas } \\
\text { terminadas. } \\
\text { - Revisión del producto backlog. }\end{array}$ \\
\hline
\end{tabular}

Nota: Se ha descrito el modo de ejecución y beneficios del Sprint Review. Elaboración Propia.

\section{Sprint Retrospective:}

Tal como indican Navarro, Fernández \& Morales (2013), es una reunión de tres horas del equipo Scrum en la que se analiza cómo fue la comunicación, el proceso y las herramientas; qué estuvo bien, qué no, y se crea un plan de mejoras para el siguiente Sprint. El tiempo, tal como en los casos anteriores, se debe ajustar proporcionalmente en el caso de proyectos de duración menor a un mes.

Tabla 89

Cuadro de Ejecución y beneficios del Sprint Retrospective

\begin{tabular}{|c|c|}
\hline MODO DE EJECUCIÓN & BENEFICIOS \\
\hline $\begin{array}{l}\text { - Se tomará una hora para realizar la } \\
\text { retrospectiva del sprint. } \\
\text { - Tanto el equipo de desarrollo como el de } \\
\text { calidad tendrá su propia retrospectiva. } \\
\text { - Se ejecutará una vez terminado el sprint } \\
\text { review y antes de realizar el siguiente sprint } \\
\text { planning. }\end{array}$ & $\begin{array}{l}\text { - Permitirá tener un autoanálisis del } \\
\text { equipo por su paso en el sprint } \\
\text { - Continuidad de lo que se hizo bien en } \\
\text { el sprint. } \\
\text { - Identificación de errores frecuentes } \\
\text { en el equipo. }\end{array}$ \\
\hline
\end{tabular}

Nota: Se ha descrito el modo de ejecución y beneficios del Sprint Retrospective. Elaboración Propia. 


\section{(1)}

\section{Planning Poker}

Según lo indicado en la web de Proyectosagiles.org ("s.f."), es una técnica que permite hacer una estimación inicial del proyecto rápida y fiable, dado que todos los miembros del equipo comparten sus diferentes informaciones y expresan su opinión sin sentirse condicionados por el resto. Cada número de la baraja significa un peso / esfuerzo / complejidad para completar un objetivo.

Tabla 90

Cuadro de Ejecución y beneficios del Planning Poker

\begin{tabular}{|l|l|}
\hline \multicolumn{1}{|c|}{ MODO DE EJECUCIÓN } & \multicolumn{1}{c|}{ BENEFICIOS } \\
\hline - Al momento de realizar las estimaciones & - $\begin{array}{l}\text { Permitirá que cada participante del } \\
\text { equipo, en base a su experiencia, pueda } \\
\text { se aplicará esta dinámica en el equipo. }\end{array}$ \\
\hline $\begin{array}{l}\text { Se realizará tanto para el equipo de } \\
\text { estimar de manera real los tiempos de }\end{array}$ \\
$\begin{array}{l}\text { desarrollo como para el equipo de } \\
\text { calidad. }\end{array}$ & $\begin{array}{l}\text { cada tarea. } \\
\text { El manejo de la baraja será de: } \\
\text { ? (indefinido), } 5 \text { (bajo), } 10 \text { (moderado), } \\
\text { 20 (medio), } 50 \text { (alto). }\end{array}$ \\
\hline
\end{tabular}

Nota: Se ha descrito el modo de ejecución y beneficios del Planning Poker. Elaboración Propia.

\section{Capacitaciones funcionales internas}

Según lo indicado por Siliceo (2001), señala que una capacitación:

Consiste en una actividad planeada y basada en necesidades reales de una empresa $u$ organización y orientada hacia un cambio en los conocimientos, habilidades y actitudes del colaborador. Es la función educativa de una empresa u organización por la cual se satisfacen necesidades presentes y se prevén necesidades futuras respecto de la preparación y habilidades de los colaboradores (p.26). 


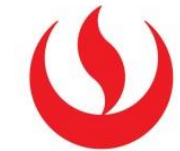

Tabla 91

Cuadro de Ejecución y beneficios de las Capacitaciones funcionales internas

\begin{tabular}{|c|c|}
\hline MODO DE EJECUCIÓN & BENEFICIOS \\
\hline $\begin{array}{l}\text { - Se necesitará mínimo de } 5 \text { participantes } \\
\text { para iniciar una capacitación funcional. } \\
\text { - Se realizará luego del horario laboral con } \\
\text { un tiempo estimado de una a dos horas } \\
\text { por día. }\end{array}$ & $\begin{array}{l}\text { - Permitirá complementar los } \\
\text { conocimientos funcionales del equipo } \\
\text { sobre un tema. } \\
\text { - Incremento de aportes sobre el tema } \\
\text { funcional. }\end{array}$ \\
\hline
\end{tabular}

Nota: Se ha descrito el modo de ejecución y beneficios de las capacitaciones funcionales internas. Elaboración Propia. 


\section{(1)}

\section{COMPOSICIÓN DE LOS GRUPOS DE TRABAJO}

Para este punto se usarán los roles que nos ofrece Scrum para ello se dará una breve explicación sobre la consistencia de cada uno de estos roles y adicionalmente, se incorporará un nuevo equipo propio de la empresa. Tal como indican Navarro, Fernández \& Morales (2013), se detalla:

El Scrum master: El cual tiene como función asegurar que el equipo está ejecutando sus prácticas, valores y normas; es el líder del equipo pero no gestiona el desarrollo.

El Product owner o dueño del producto: El cual representa a los interesados, tiene la función de maximizar el valor del producto y el trabajo del equipo de desarrollo; de gestionar la lista de funcionalidades requeridas o Product Backlog.

El Development Team o equipo de desarrollo: El cual tiene como función convertir lo que el cliente quiere, en iteraciones funcionales del producto; dicho equipo no tiene jerarquías, todos sus miembros.

Para realizar el desarrollo y la implementación del proyecto presentado anteriormente, el objeto de estudio evaluará y determinará que proveedor se hará cargo de este producto. Como parte de los requisitos para los postulantes, el objeto de estudio definirá la composición de los grupos de trabajo tanto del lado del proveedor como del mismo. Esta definición es fundamental ya que el objeto de estudio deberá dar las definiciones, requisitos, gestionar los tiempos y certificación de la nueva solución. A continuación, se detalla la composición que deberá presentar el grupo de trabajo explicando las habilidades blandas, técnicas y funciones a cumplir de igual forma el perfil que deberá tener y la cantidad necesaria:

Tabla 92

Cuadro de habilidades del Product Owner (Objeto de Estudio)

\begin{tabular}{|c|c|c|}
\hline Habilidades Blandas & Habilidades Técnicas & Funciones \\
\hline $\begin{array}{l}\text { - Paciencia } \\
\text { - Trabajo en equipo }\end{array}$ & $\begin{array}{l}\text { - Conocimiento del proceso } \\
\text { de Gestión de Proyectos }\end{array}$ & $\begin{array}{l}\text { - Brindar las especificaciones } \\
\text { funcionales del producto }\end{array}$ \\
\hline
\end{tabular}




\section{(4)}

\begin{tabular}{|c|c|c|}
\hline Habilidades Blandas & Habilidades Técnicas & Funciones \\
\hline $\begin{array}{ll}\text { - } & \text { Comunicativo } \\
\text { - } & \text { Decisivo } \\
\text { - } & \text { Poder de } \\
\text { negociación } \\
\text { - Exigente }\end{array}$ & $\begin{array}{l}\text { - Experiencia en el } \\
\text { desempeño de gestión } \\
\text { - Conocimiento sobre los } \\
\text { artefactos de Scrum }\end{array}$ & 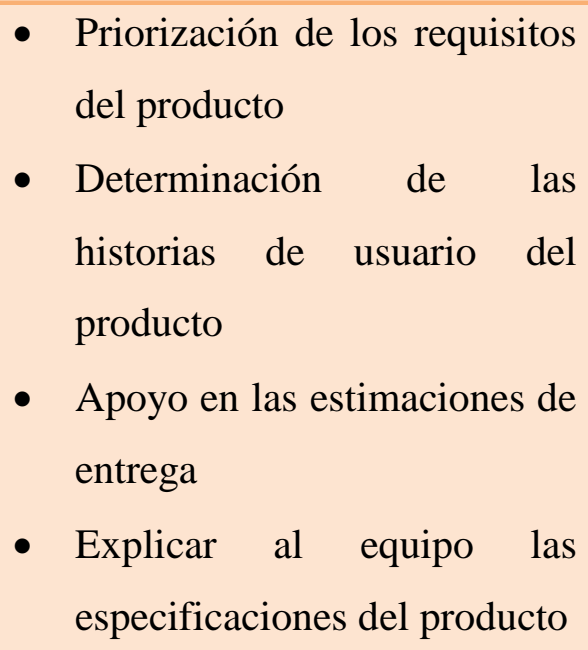 \\
\hline $\begin{array}{l}\text { Perfil y cantidad del } \\
\text { personal }\end{array}$ & - Un líder técnico & \\
\hline
\end{tabular}

Nota: Se ha descrito las habilidades blandas, técnicas y las funciones del Product Owner del Objeto de Estudio. Adaptado de "Manual de funciones y perfiles de puestos", por El Objeto de Estudio, 2017.

Tabla 93

Cuadro de habilidades del Scrum Master (Proveedor)

\begin{tabular}{|c|c|c|}
\hline Habilidades Blandas & Habilidades Técnicas & Funciones \\
\hline $\begin{array}{ll}\text { - } & \text { Comunicativo } \\
\text { - } & \text { Paciente } \\
\text { - } & \text { Trabajo en equipo } \\
\text { - } & \text { Servicial } \\
\text { - } & \text { Carismático } \\
\text { - } & \text { Liderazgo }\end{array}$ & $\begin{array}{l}\text { - Amplio conocimiento de } \\
\text { - Ecrum } \\
\text { Experiencia en desarrollo } \\
\text { de proyectos con Scrum } \\
\text { - Experiencia en } \\
\text { implementación de la } \\
\text { herramienta JIRA }\end{array}$ & $\begin{array}{l}\text { - Coordinación entre el equipo } \\
\text { de desarrollo y el product } \\
\text { owner } \\
\text { - Estimación de los entregables } \\
\text { - Facilitar las herramientas } \\
\text { necesarias al equipo } \\
\text { - Apoyo al equipo de } \\
\text { desarrollo }\end{array}$ \\
\hline $\begin{array}{l}\text { Perfil y cantidad del } \\
\text { personal }\end{array}$ & - Un & \\
\hline
\end{tabular}

Nota: Se ha descrito las habilidades blandas, técnicas y las funciones del Scrum Master del Proveedor. Adaptado de "Manual de funciones y perfiles de puestos", por El Objeto de Estudio, 2017. 


\section{$(4)$}

Tabla 94

Cuadro de habilidades del Scrum Master (Objeto de Estudio)

\begin{tabular}{|c|c|c|}
\hline Habilidades Blandas & Habilidades Técnicas & Funciones \\
\hline $\begin{array}{ll}\text { - } & \text { Comunicativo } \\
\text { - } & \text { Paciente } \\
\text { - } & \text { Trabajo en equipo } \\
\text { - } & \text { Servicial } \\
\text { - } & \text { Carismático } \\
\text { - } & \text { Liderazgo }\end{array}$ & $\begin{array}{l}\text { - Amplio conocimiento de } \\
\text { Scrum } \\
\text { - Experiencia en desarrollo de } \\
\text { proyectos con Scrum } \\
\text { - Conocimiento en gestión de } \\
\text { proyectos }\end{array}$ & 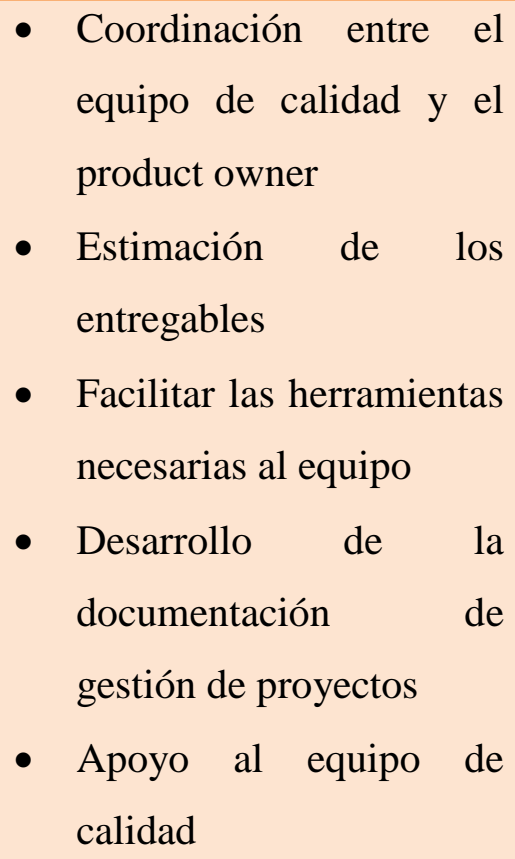 \\
\hline $\begin{array}{l}\text { Perfil y cantidad del } \\
\text { personal }\end{array}$ & - Un analist & \\
\hline
\end{tabular}

Nota: Se ha descrito las habilidades blandas, técnicas y las funciones del Scrum Master del Objeto de Estudio. Adaptado de "Manual de funciones y perfiles de puestos", por El Objeto de Estudio, 2017.

Tabla 95

Cuadro de habilidades del Development Team (Proveedor)

\begin{tabular}{|c|c|c|}
\hline Habilidades Blandas & Habilidades Técnicas & Funciones \\
\hline $\begin{array}{l}\text { - Trabajo en equipo } \\
\text { - Proactivo } \\
\text { - Comprometido } \\
\text { - Autogestionable } \\
\text { - Intuitivos }\end{array}$ & $\begin{array}{l}\text { - Conocimiento técnico en } \\
\text { lenguaje de programación } \\
\text { JAVA } \\
\text { - Conocimiento técnico de la } \\
\text { herramienta JIRA Editor } \\
\text { - Conocimiento técnico del } \\
\text { modelo de datos del JIRA }\end{array}$ & $\begin{array}{l}\text { - Desarrollo de los } \\
\text { requisitos del product } \\
\text { owner } \\
\text { - } \text { Entendimiento de los } \\
\text { requisitos del product } \\
\text { owner } \\
\text { - Participar en las } \\
\text { reuniones del equipo y }\end{array}$ \\
\hline
\end{tabular}




\section{(1)}

\begin{tabular}{|l|l|l|}
\hline Habilidades Blandas & \multicolumn{1}{|c|}{ Habilidades Técnicas } & \multicolumn{1}{c|}{ Funciones } \\
\hline & $\begin{array}{l}\text { Experiencia en base de datos } \\
\text { MySQL }\end{array}$ & $\begin{array}{l}\text { del cliente } \\
\text { Brindar apoyo mutuo al } \\
\text { equipo }\end{array}$ \\
\hline $\begin{array}{l}\text { Perfil y cantidad del } \\
\text { personal }\end{array}$ & $\bullet$ & \begin{tabular}{l} 
Dos analistas programadores senior front end \\
\hline
\end{tabular} \\
\hline
\end{tabular}

Nota: Se ha descrito las habilidades blandas, técnicas y las funciones del Development Team del Proveedor.

Adaptado de "Manual de funciones y perfiles de puestos", por El Objeto de Estudio, 2017.

Tabla 96

Cuadro de habilidades del Quality Team (Objeto de Estudio)

\begin{tabular}{|c|c|c|}
\hline Habilidades Blandas & Habilidades Técnicas & Funciones \\
\hline $\begin{array}{l}\text { - } \text { Trabajo en equipo } \\
\text { - } \text { Proactivo } \\
\text { - Comprometido } \\
\text { - Autogestionable } \\
\text { - Intuitivos } \\
\text { - Exigentes } \\
\text { - } \text { Minuciosos }\end{array}$ & $\begin{array}{l}\text { - Conocimiento del proceso de } \\
\text { Gestión de Proyectos } \\
\text { - Experiencia en evaluación de } \\
\text { calidad de productos } \\
\text { - Conocimiento de base de } \\
\text { datos MySQL } \\
\text { - Experiencia } \\
\text { automatización de pruebas }\end{array}$ & $\begin{array}{l}\text { - } \text { Verificar la } \\
\text { documentación entregada } \\
\text { por el proveedor } \\
\text { - Validar la documentación } \\
\text { entregada por el proveedor } \\
\text { - Diseñar las pruebas del } \\
\text { producto } \\
\text { - Ejecutar los escenarios de } \\
\text { prueba del producto } \\
\text { Elaboración de pruebas } \\
\text { automatizadas. } \\
\text { Ejecución de pruebas } \\
\text { manuales. }\end{array}$ \\
\hline $\begin{array}{l}\text { Perfil y cantidad del } \\
\text { personal }\end{array}$ & $\begin{array}{l}\text { - Un analista de calidad senior } \\
\text { - Dos analistas de pruebas seni }\end{array}$ & \\
\hline
\end{tabular}

Nota: Se ha descrito las habilidades blandas, técnicas y las funciones del Quality Team del Objeto de Estudio.

Adaptado de "Manual de funciones y perfiles de puestos", por El Objeto de Estudio, 2017. 


\section{(1)}

El costo total mensual de todo el grupo de trabajo se detalla en la siguiente tabla:

Tabla 97

Cuadro de Costo Total del Equipo

\begin{tabular}{|l|r|r|r|r|}
\hline \multicolumn{1}{|c|}{ Perfil } & Cantidad & Costo (S/.) & $\begin{array}{r}\text { Horas } \\
\text { (Mes) }\end{array}$ & \multicolumn{1}{|c|}{ Total } \\
\hline Líder técnico & 1 & 37.50 & 160 & $6,000.00$ \\
\hline Analista de sistemas senior & 2 & 34.40 & 320 & $11,008.00$ \\
\hline Analista programador senior front end & 2 & 31.25 & 320 & $10,000.00$ \\
\hline Analista de base de datos MySQL & 1 & 31.25 & 160 & $5,000.00$ \\
\hline Analista de calidad senior & 1 & 31.25 & 160 & $5,000.00$ \\
\hline Analista de pruebas senior & 2 & 31.25 & 320 & $10,000.00$ \\
\hline Costo total (S/.) & & & & $\mathbf{4 7 , 0 0 8 . 0 0}$ \\
\hline
\end{tabular}

Nota: Se ha descrito la cantidad, costo horas y total de cada perfil. Elaboración Propia.

\section{DEFINICIÓN DE LAS HERRAMIENTAS A UTILIZAR}

Para este proyecto se va aplicar las siguientes herramientas que nos ofrece Scrum, para ello se brindará un pequeño concepto de cada uno de estas, la forma en cómo se emplearán y lo que se realizará.

\section{Definition of Done:}

Tal como indican Schwaber \& Sutherland (2016), es importante en Scrum esta definición, es decir cuando un elemento de la Lista de Producto o un Incremento se describe como "Terminado", todo el mundo debe entender lo que significa "Terminado". Aunque esto varía significativamente para cada Equipo Scrum, los miembros del Equipo deben tener un entendimiento compartido de lo que significa que el trabajo esté terminado para asegurar la transparencia.

El Equipo Scrum lo utiliza para evaluar cuándo se ha completado el trabajo sobre el Incremento de producto. Esta misma definición guía al Equipo de Desarrollo en saber cuántos elementos de la Lista de Producto puede seleccionar durante la Planificación del Sprint. El 


\section{(1)}

propósito de cada sprint es entregar Incrementos de funcionalidad que potencialmente se puedan poner en producción y que se ajustan a la Definición de "Terminado" actual del Equipo Scrum.

Si hay múltiples Equipos Scrum trabajando en la entrega del sistema o producto, los equipos de desarrolladores en todos los Equipos Scrum deben definir en conjunto la definición de "Terminado". Cualquier producto o sistema debería tener una definición de "Terminado" que es un estándar para cualquier trabajo realizado sobre él.

Para el equipo de desarrollo se definió:

Tabla 98

Cuadro de Criterios para el Equipo de Desarrollo

\begin{tabular}{|c|l|}
\hline Código de criterio & Criterio de tarea terminada para desarrollo \\
\hline CTTD001 & Ejecución de pruebas unitarias \\
\hline CTTD002 & Ejecución de pruebas funcionales \\
\hline CTTD003 & Funcionalidad sin bugs \\
\hline CTTD004 & Entorno preparado para despliegue \\
\hline
\end{tabular}

Nota: Se ha descrito el código y descripción de cada criterio del Equipo de Desarrollo. Elaboración Propia.

Para el equipo de calidad se definió:

Tabla 99

Cuadro de Criterios para el Equipo de Calidad

\begin{tabular}{|c|l|}
\hline Código de criterio & Criterio de tarea terminada para calidad \\
\hline CTTC001 & Elaboración de casos de prueba \\
\hline CTTC002 & Ejecución de pruebas integrales \\
\hline CTTC003 & Elaboración de prueba automatizada \\
\hline CTTC004 & Ejecución de pruebas de regresión \\
\hline CTTC005 & Uso en los diferentes navegadores (Chrome, Mozilla, IE) \\
\hline
\end{tabular}

Nota: Se ha descrito el código y descripción de cada criterio del Equipo de Calidad. Elaboración Propia. 


\section{$(\varphi$}

\section{Historias de Usuario:}

Tal como indican Canós, Penadés \& Letelier (2012), son la técnica utilizada para especificar los requisitos del software. Se trata de tarjetas de papel en las cuales el cliente describe brevemente las características que el sistema debe poseer, sean requisitos funcionales o no funcionales. El tratamiento de las historias de usuario es muy dinámico y flexible. Cada historia de usuario es lo suficientemente comprensible y delimitada para que los programadores puedan implementarla en unas semanas.

Tabla 100

Cuadro de Historias de Usuario

\begin{tabular}{|c|c|c|c|c|}
\hline Código & Nombre & Objetivo & $\begin{array}{l}\text { Condición de } \\
\text { Satisfacción }\end{array}$ & Esfuerzo \\
\hline HU001 & $\begin{array}{l}\text { Autenticación } \\
\text { al sistema }\end{array}$ & $\begin{array}{l}\text { El usuario podrá } \\
\text { autenticarse al sistema con } \\
\text { el objetivo de utilizarlo. }\end{array}$ & $\begin{array}{l}\text { - Ingreso al sistema } \\
\text { mediante el dominio } \\
\text { de red. }\end{array}$ & 5 \\
\hline HU002 & $\begin{array}{l}\text { Creación de } \\
\text { dashboard }\end{array}$ & $\begin{array}{l}\text { El usuario podrá contar con } \\
\text { un panel personalizado por } \\
\text { la empresa con el fin tener } \\
\text { una visibilidad de todos los } \\
\text { proyectos y tareas. }\end{array}$ & $\begin{array}{l}\text { - Visualizar las tareas } \\
\text { asignadas. } \\
\text { - Visualizar los } \\
\text { proyectos en curso. }\end{array}$ & 5 \\
\hline HU003 & $\begin{array}{l}\text { Administrar } \\
\text { flujos de } \\
\text { trabajo }\end{array}$ & $\begin{array}{l}\text { El usuario podrá definir los } \\
\text { flujos de trabajo para los } \\
\text { proyectos, tareas y entre } \\
\text { otros con el objetivo de } \\
\text { tener un estandar de trabajo. }\end{array}$ & $\begin{array}{l}\text { - } \text { Flujos claros y } \\
\text { definidos por la } \\
\text { empresa } \\
\text { - Flujos independientes } \\
\text { por cada tipo de } \\
\text { trabajo. }\end{array}$ & 20 \\
\hline HU004 & $\begin{array}{l}\text { Crear } \\
\text { proyecto }\end{array}$ & $\begin{array}{l}\text { El usuario podrá crear } \\
\text { proyectos con el objetivo de } \\
\text { podar gestionar el flujo del } \\
\text { proyecto. }\end{array}$ & $\begin{array}{l}\text { - Crear los } 3 \text { tipos de } \\
\text { proyectos que ofrece } \\
\text { JIRA. }\end{array}$ & 5 \\
\hline HU005 & Crear tareas & El usuario podrá crear tareas & - Tareas diferenciadas & 5 \\
\hline
\end{tabular}




\section{(1)}

\begin{tabular}{|c|c|c|c|c|}
\hline Código & Nombre & Objetivo & $\begin{array}{l}\text { Condición de } \\
\text { Satisfacción }\end{array}$ & Esfuerzo \\
\hline & & $\begin{array}{l}\text { con el objetivo de } \\
\text { administrar las tareas que se } \\
\text { realizará en cada proyecto. }\end{array}$ & $\begin{array}{l}\text { por tipo de tarea. } \\
\text { Tareas relacionadas de } \\
\text { manera correcta al } \\
\text { proyecto. }\end{array}$ & \\
\hline HU006 & $\begin{array}{l}\text { Asignar } \\
\text { responsables }\end{array}$ & $\begin{array}{l}\text { El usuario podrá asignar } \\
\text { responsables a cada tarea } \\
\text { con el objetivo de que el } \\
\text { responsable pueda visualizar } \\
\text { y atender la tarea. }\end{array}$ & $\begin{array}{l}\text { - Lista de responsables } \\
\text { en base a los recursos } \\
\text { de la empresa. } \\
\text { - Asignación correcta y } \\
\text { visualización de la } \\
\text { tarea en la bandeja del } \\
\text { responsable. }\end{array}$ & 10 \\
\hline HU007 & $\begin{array}{l}\text { Crear gráficos } \\
\text { estadísticos }\end{array}$ & $\begin{array}{l}\text { El usuario podrá generar } \\
\text { gráficos estadísticos con el } \\
\text { objetivo de } \\
\text { información precisa del } \\
\text { estado y avance de los } \\
\text { proyectos y tareas. }\end{array}$ & $\begin{array}{l}\text { - Gráficos claros con } \\
\text { información } \\
\text { consistente. } \\
\text { - Diversos gráficos de } \\
\text { explotación de } \\
\text { información como } \\
\text { barras, circulares, } \\
\text { histogramas, etc. }\end{array}$ & 50 \\
\hline HU008 & $\begin{array}{l}\text { Personalizar } \\
\text { filtros por } \\
\text { tareas } \quad \text { o } \\
\text { proyectos }\end{array}$ & $\begin{array}{l}\text { El usuario podrá crear filtros } \\
\text { de búsqueda personalizados } \\
\text { como accesos directos con } \\
\text { el objetivo de identificar de } \\
\text { manera más rápida y } \\
\text { oportuna sus tareas y } \\
\text { proyectos. }\end{array}$ & $\begin{array}{l}\text { - Creación de acceso } \\
\text { directo en el panel de } \\
\text { trabajo. }\end{array}$ & 5 \\
\hline HU009 & $\begin{array}{l}\text { Carga de datos } \\
\text { en proyectos }\end{array}$ & $\begin{array}{l}\text { El usuario podrá ingresar los } \\
\text { datos de cada proyecto con } \\
\text { el objetivo de tener toda la }\end{array}$ & $\begin{array}{l}\text { - Datos definidos por la } \\
\text { empresa por cada tipo } \\
\text { de proyecto. }\end{array}$ & 20 \\
\hline
\end{tabular}




\section{(1)}

\begin{tabular}{|c|c|c|c|c|}
\hline Código & Nombre & Objetivo & $\begin{array}{l}\text { Condición de } \\
\text { Satisfacción }\end{array}$ & Esfuerzo \\
\hline & & $\begin{array}{l}\text { información obligatoria o } \\
\text { complementaria que trabaja } \\
\text { la empresa para cada } \\
\text { proyecto. }\end{array}$ & $\begin{array}{l}\text { - Campos obligatorios y } \\
\text { opcionales definidos. }\end{array}$ & \\
\hline HU010 & $\begin{array}{l}\text { Carga de datos } \\
\text { en tareas }\end{array}$ & $\begin{array}{l}\text { El usuario podrá ingresar los } \\
\text { datos de cada tarea con el } \\
\text { objetivo de tener toda la } \\
\text { información obligatoria o } \\
\text { complementaria que trabaja } \\
\text { la empresa para cada tarea. }\end{array}$ & $\begin{array}{l}\text { - Datos definidos por la } \\
\text { empresa por cada tipo } \\
\text { de tarea. } \\
\text { - Campos obligatorios y } \\
\text { opcionales definidos. }\end{array}$ & 20 \\
\hline HU011 & $\begin{array}{l}\text { Personalizació } \\
\mathrm{n} \text { de la } \\
\text { empresa en el } \\
\text { sistema }\end{array}$ & $\begin{array}{l}\text { El usuario podrá ver los } \\
\text { datos de la empresa en el } \\
\text { sistema con el objetivo de } \\
\text { identificar que el producto } \\
\text { ya se encuentra } \\
\text { personalizado. }\end{array}$ & $\begin{array}{llll}\text { - } & \text { Logos y } & \text { títulos } \\
\text { relacionados } & \text { a } & \text { la } \\
\text { empresa. } & & & \end{array}$ & 5 \\
\hline HU012 & $\begin{array}{l}\text { Paneles por } \\
\text { JEditor }\end{array}$ & $\begin{array}{l}\text { El usuario podrá diseñar } \\
\text { nuevos paneles del JIRA } \\
\text { con el objetivo de poder } \\
\text { emplearlas en el trabajo } \\
\text { diario de la herramienta. }\end{array}$ & $\begin{array}{llr}\text { - Campos } & \text { necesarios } \\
\text { desde la } & \text { opción } \\
\text { JEditor. } & & \end{array}$ & 10 \\
\hline HU013 & $\begin{array}{l}\text { Funcionalidad } \\
\text { del sistema por } \\
\text { nuevos plugins }\end{array}$ & $\begin{array}{l}\text { El usuario podrá acoplar } \\
\text { nuevos plugins al JIRA con } \\
\text { el objetivo de poder contar } \\
\text { con nuevos entornos que se } \\
\text { ofrecen en el market de la } \\
\text { herramienta. }\end{array}$ & $\begin{array}{l}\text { Acoplamiento correcto } \\
\text { de los nuevos plugins } \\
\text { que ofrece el sistema. }\end{array}$ & 10 \\
\hline HU014 & $\begin{array}{l}\text { Comentarios } \\
\text { ordenados por }\end{array}$ & $\begin{array}{l}\text { El usuario podrá visualizar } \\
\text { las actividades realizadas en }\end{array}$ & $\begin{array}{l}\text { - Bitácora de trabajo } \\
\text { ordenada y coherente. }\end{array}$ & 10 \\
\hline
\end{tabular}




\section{()}

\begin{tabular}{|c|c|c|c|c|}
\hline Código & Nombre & Objetivo & $\begin{array}{l}\text { Condición de } \\
\text { Satisfacción }\end{array}$ & Esfuerzo \\
\hline & $\begin{array}{l}\text { proyecto } \quad 0 \\
\text { tareas }\end{array}$ & $\begin{array}{l}\text { cada tarea con el objetivo de } \\
\text { conocer lo que hizo cada } \\
\text { responsable en todo el } \\
\text { tiempo de vida de la tarea. }\end{array}$ & $\begin{array}{l}\text { - Orden cronológico de } \\
\text { las actividades } \\
\text { realizadas. } \\
\text { - Archivos adjuntos } \\
\text { subidos por cada } \\
\text { responsable. } \\
\text { - Modificaciones } \\
\text { realizadas en el detalle } \\
\text { de la tarea o } \\
\text { comentarios. }\end{array}$ & \\
\hline HU015 & $\begin{array}{l}\text { Relación entre } \\
\text { proyectos y } \\
\text { tareas por link }\end{array}$ & $\begin{array}{l}\text { El usuario podrá relacionar } \\
\text { de manera directa las tareas } \\
\text { a cada proyecto o las tareas } \\
\text { entre tareas con el fin de } \\
\text { tener un mapeo rápido de } \\
\text { las tareas y los proyectos } \\
\text { relacionados. }\end{array}$ & $\begin{array}{l}\text { - Accesos rápidos entre } \\
\text { tareas y proyectos } \\
\text { - Nombre y título de la } \\
\text { tarea relacionada. }\end{array}$ & 5 \\
\hline HU016 & $\begin{array}{l}\text { Generación de } \\
\text { tableros } \\
\text { Scrum/Kanban }\end{array}$ & $\begin{array}{l}\text { El usuario podrá generar } \\
\text { tableros de tipo Scrum o } \\
\text { Kanban con el objetivo de } \\
\text { saber el estado de cada tarea } \\
\text { en cada tablero. }\end{array}$ & $\begin{array}{l}\text { - Flujos establecidos por } \\
\text { la empresa volcados en } \\
\text { el tablero } \\
\text { - Visualización de todas } \\
\text { las tareas } \\
\text { responsables } \\
\text { relacionadas a un } \\
\text { proyecto. }\end{array}$ & 10 \\
\hline HU017 & $\begin{array}{lr}\begin{array}{lr}\text { Niveles } & \text { de } \\
\text { acceso } & \text { por } \\
\text { proyecto } & \text { o } \\
\text { tareas } & \end{array}\end{array}$ & $\begin{array}{l}\text { El usuario podrá asignar } \\
\text { niveles de acceso por } \\
\text { proyecto o tareas con el } \\
\text { objetivo de restringir el }\end{array}$ & $\begin{array}{l}\text { - Lista de áreas } \\
\text { definidas por la } \\
\text { empresa } \\
\text { - Niveles de acceso }\end{array}$ & 10 \\
\hline
\end{tabular}




\section{(1)}

\begin{tabular}{|c|c|c|c|c|}
\hline Código & Nombre & Objetivo & $\begin{array}{l}\text { Condición de } \\
\text { Satisfacción }\end{array}$ & Esfuerzo \\
\hline & & $\begin{array}{l}\text { acceso y visualización de } \\
\text { los poryectos o tareas } \\
\text { diferenciandolo por áreas o } \\
\text { algun otro criterio. }\end{array}$ & $\begin{array}{l}\text { definidas por la } \\
\text { empresa. }\end{array}$ & \\
\hline HU018 & $\begin{array}{l}\text { Administrar } \\
\text { los tipos de } \\
\text { tareas }\end{array}$ & $\begin{array}{l}\text { El usuario podrá administrar } \\
\text { sus tipos de tarea con el } \\
\text { objetivo de tener } \\
\text { estandarizado los tipos de } \\
\text { tarea a realizar. }\end{array}$ & $\begin{array}{l}\text { - Tipo de tareas } \\
\text { diferenciadas por ícono } \\
\text { y descripción. } \\
\text { - Descripción del tipo de } \\
\text { tarea precisa definida } \\
\text { por la empresa. }\end{array}$ & 10 \\
\hline HU019 & $\begin{array}{l}\text { Búsquedas } \\
\text { basadas en } \\
\text { lenguaje del } \\
\text { sistema }\end{array}$ & $\begin{array}{l}\text { El usuario podrá realizar } \\
\text { sentencias de búsqueda } \\
\text { basadas en el lenguaje de la } \\
\text { herramienta con el objetivo } \\
\text { de poder explotar mejor los } \\
\text { filtros de información. }\end{array}$ & $\begin{array}{l}\text { - Autollenado de la } \\
\text { sentencia. } \\
\text { - Autollenado de los } \\
\text { posibles parámetros de } \\
\text { búsqueda. }\end{array}$ & 10 \\
\hline HU020 & $\begin{array}{l}\text { Adición de } \\
\text { archivos } \\
\text { adjuntos }\end{array}$ & $\begin{array}{l}\text { El usuario podrá adjuntar } \\
\text { archivos a cada tarea con el } \\
\text { fin de contar con un único } \\
\text { repositorio de información. }\end{array}$ & $\begin{array}{l}\text { - } \text { Podrá adjuntar } \\
\text { archivos de cualquier } \\
\text { tipo de extensión. } \\
\text { - Peso máximo de cada } \\
\text { adjunto 3MB. }\end{array}$ & 5 \\
\hline HU021 & $\begin{array}{l}\text { Notificaciones } \\
\text { por cambios en } \\
\text { tareas o } \\
\text { proyectos }\end{array}$ & $\begin{array}{l}\text { El usuario podrá recibir } \\
\text { notificaciones por cambios } \\
\text { en las tareas o proyectos con } \\
\text { el fin de estar al tanto de los } \\
\text { eventos que se generan. }\end{array}$ & $\begin{array}{l}\text { - Notificaciones a los } \\
\text { involucrados en la } \\
\text { tarea o proyecto } \\
\text { - Notificación al correo } \\
\text { de cada involucrado. }\end{array}$ & 10 \\
\hline HU022 & $\begin{array}{l}\text { Procesamiento } \\
\text { de } \\
\text { cronogramas }\end{array}$ & $\begin{array}{l}\text { El usuario podrá ejecutar un } \\
\text { procesamiento de } \\
\text { cronograma con el objetivo }\end{array}$ & $\begin{array}{l}\text { - Generación del } \\
\text { informe de manera }\end{array}$ & 50 \\
\hline
\end{tabular}




\section{(4)}

\begin{tabular}{|c|c|c|c|c|}
\hline Código & Nombre & Objetivo & $\begin{array}{l}\text { Condición de } \\
\text { Satisfacción }\end{array}$ & Esfuerzo \\
\hline & $\begin{array}{l}\text { de forma } \\
\text { semanal }\end{array}$ & $\begin{array}{l}\text { de generar un informe } \\
\text { comparativo entre un } \\
\text { proyecto a una fecha de } \\
\text { corte "X" contra el actual. } \\
\text { Adicionalmente, el sistema } \\
\text { realizará } \\
\text { procesamiento en forma } \\
\text { programada una vez por } \\
\text { semana. }\end{array}$ & $\begin{array}{l}\text { asíncrona. } \\
\text { - Curva de desviación } \\
\text { del proyecto o tarea. }\end{array}$ & \\
\hline HU023 & $\begin{array}{l}\text { Matriz de } \\
\text { Trazabilidad }\end{array}$ & $\begin{array}{l}\text { El usuario podrá registrar / } \\
\text { actualizar la matriz de } \\
\text { trazabilidad. }\end{array}$ & $\begin{array}{l}\text { - Visualización en } \\
\text { tiempo real de la } \\
\text { matriz de trazabilidad. } \\
\text { - Generación de reporte } \\
\text { de matriz de } \\
\text { trazabilidad. }\end{array}$ & 20 \\
\hline HU024 & $\begin{array}{ll}\text { Lista de } \\
\text { Riesgos }\end{array}$ & $\begin{array}{l}\text { El usuario podrá registrar / } \\
\text { actualizar la lista de riesgos. }\end{array}$ & $\begin{array}{l}\text { - Visualización } \\
\text { tiempo real de la lista } \\
\text { de riesgos. } \\
\text { - Generación de reporte } \\
\text { de la lista de riesgos. }\end{array}$ & 20 \\
\hline HU025 & $\begin{array}{l}\text { Bitácora de } \\
\text { Seguimiento }\end{array}$ & $\begin{array}{l}\text { El usuario podrá registrar / } \\
\text { actualizar la bitácora de } \\
\text { seguimiento. }\end{array}$ & $\begin{array}{l}\text { - Visualización } \\
\text { tiempo real de la } \\
\text { bitácora } \\
\text { seguimiento } \\
\text { - Generación de reporte } \\
\text { de la bitácora de } \\
\text { seguimiento }\end{array}$ & 20 \\
\hline
\end{tabular}

Nota: Se ha descrito el código, nombre, objetivo, condición de satisfacción y esfuerzo por cada Historia de usuario.

Elaboración Propia. 


\section{(1)}

\section{Product Backlog:}

Tal como indican Navarro, Fernández \& Morales (2013), es una lista ordenada por valor, riesgo, prioridad y necesidad de los requerimientos que el dueño del producto define, actualiza y ordena. La lista tiene como característica particular que nunca está terminada, pues evoluciona durante el desarrollo del proyecto.

El esfuerzo para cada historia de usuario ya fue definido por el equipo de trabajo y el esfuerzo de la documentación será definido por el Scrum master. Ahora el dueño del producto asignará la puntuación del valor y riesgo usando la baraja del Planning Poker. Teniendo el puntaje de cada criterio se colocará la prioridad de cada historia de usuario o tarea.

Tabla 101

Cuadro del Product Backlog

\begin{tabular}{|l|c|c|c|c|}
\hline \multicolumn{1}{|c|}{ Tarea } & Esfuerzo & Valor & Riesgo & Prioridad \\
\hline Elaboración de HU001 & 5 & 50 & 5 & 1 \\
\hline Elaboración de HU002 & 5 & 50 & 5 & 3 \\
\hline Elaboración de HU003 & 20 & 50 & 10 & 11 \\
\hline Elaboración de HU004 & 5 & 50 & 5 & 5 \\
\hline Elaboración de HU005 & 5 & 50 & 5 & 9 \\
\hline Elaboración de HU006 & 10 & 20 & 5 & 15 \\
\hline Elaboración de HU007 & 50 & 20 & 20 & 20 \\
\hline Elaboración de HU008 & 5 & 10 & 5 & 22 \\
\hline Elaboración de HU009 & 20 & 50 & 10 & 13 \\
\hline Elaboración de HU010 & 20 & 50 & 10 & 14 \\
\hline Elaboración de HU011 & 5 & 20 & 5 & 18 \\
\hline Elaboración de HU012 & 10 & 10 & 20 & 28 \\
\hline Elaboración de HU013 & 10 & 10 & 10 & 27 \\
\hline Elaboración de HU014 & 10 & 20 & 10 & 19 \\
\hline Elaboración de HU015 & 5 & 10 & 10 & 24 \\
\hline Elaboración de HU016 & 10 & 20 & 5 & 17 \\
\hline Elaboración de HU017 & 10 & 10 & 5 & 21 \\
\hline
\end{tabular}




\section{()}

\begin{tabular}{|c|c|c|c|c|}
\hline Tarea & Esfuerzo & Valor & Riesgo & Prioridad \\
\hline Elaboración de HU018 & 10 & 50 & 5 & 7 \\
\hline Elaboración de HU019 & 10 & 10 & 10 & 26 \\
\hline Elaboración de HU020 & 5 & 10 & 10 & 25 \\
\hline Elaboración de HU021 & 10 & 10 & 5 & 23 \\
\hline Elaboración de HU022 & 50 & 50 & 20 & 16 \\
\hline Elaboración de HU023 & 20 & 20 & 10 & 29 \\
\hline Elaboración de HU024 & 20 & 20 & 10 & 30 \\
\hline Elaboración de HU025 & 20 & 20 & 10 & 31 \\
\hline Certificación de la HU001 & 20 & 50 & 50 & 2 \\
\hline Certificación de la HU002 & 20 & 50 & 50 & 4 \\
\hline Certificación de la HU004 & 20 & 50 & 50 & 6 \\
\hline Certificación de la HU018 & 20 & 50 & 50 & 8 \\
\hline Certificación de la HU005 & 20 & 50 & 50 & 10 \\
\hline Certificación de la HU003 & 20 & 50 & 50 & 12 \\
\hline $\begin{array}{l}\text { Elaborar el documento de Lista de } \\
\text { Personalización }\end{array}$ & 5 & 5 & 5 & 32 \\
\hline $\begin{array}{l}\text { Elaborar el documento de Análisis de } \\
\text { Requerimientos (Product Backlog) }\end{array}$ & 10 & 20 & 5 & 33 \\
\hline Elaborar el Sprint Backlog & 10 & 20 & 5 & 34 \\
\hline $\begin{array}{l}\text { Elaborar el documento de Análisis y Diseño } \\
\text { del Producto (Historias de Usuario) }\end{array}$ & 10 & 20 & 5 & 35 \\
\hline $\begin{array}{l}\text { Elaborar el Manual de Actualización de } \\
\text { Versión }\end{array}$ & 10 & 10 & 10 & 36 \\
\hline $\begin{array}{l}\text { Elaborar el documento de Repositorio de } \\
\text { Pruebas }\end{array}$ & 10 & 10 & 10 & 37 \\
\hline Elaborar el Informe de Certificación & 10 & 10 & 10 & 38 \\
\hline Elaborar el documento de Implementación & 10 & 10 & 20 & 39 \\
\hline
\end{tabular}

Nota: Se ha descrito el código/tarea, esfuerzo, valor, riesgo y prioridad de cada Historia de usuario/Tarea.

Elaboración Propia. 


\section{(1)}

\section{Sprint Backlog:}

Tal como indican Navarro, Fernández \& Morales (2013), es un subconjunto de ítems del Product Backlog y el plan para realizar en el Incremento del producto. Debido a que el Product backlog está organizado por prioridad, el Sprint backlog es construido con los requerimientos más prioritarios del Product backlog y con aquellos que quedaron por resolver en el Sprint anterior (en caso aplique). Una vez construido, el Sprint backlog debe ser aceptado por el equipo de desarrollo, pertenece a éste y solo puede ser modificado por él. Requerimientos adicionales deben ser incluidos en el Product backlog y desarrollados en el siguiente Sprint, si su prioridad así lo indica.

Se priorizó las tareas y el Sprint 1 solo contendrá la elaboración y certificación de 6 Historias de Usuario.

Tabla 102

Cuadro del Sprint Backlog del Sprint 1

\begin{tabular}{|c|l|c|l|}
\hline Código & \multicolumn{1}{|c|}{ Tarea } & Esfuerzo & \multicolumn{1}{|c|}{ Responsable } \\
\hline Sprint & & & \\
\hline S01 & Elaboración de HU001 & 5 & Analista programador senior front end 1 \\
\hline S01 & Certificación de la HU001 & 5 & Analista de pruebas senior 1 \\
\hline S01 & Claboración de HU002 & 5 & Analista programador senior front end 2 \\
\hline S01 & Elaboración de HU004 & 5 & Analista programador senior front end 1 \\
\hline S01 & Certificación de la HU004 & 5 & Analista de pruebas senior 1 \\
\hline S01 & Elaboración de HU018 & 10 & Analista de base de datos MySQL \\
\hline S01 & Certificación de la HU018 & 10 & Analista de pruebas senior 2 \\
\hline S01 & Elaboración de HU005 & 5 & Analista programador senior front end 2 \\
\hline S01 & Certificación de la HU005 & 5 & Analista de pruebas senior 1 \\
\hline S01 & Elaboración de HU003 & 20 & Analista de base de datos MySQL \\
\hline S01 & Certificación de la HU003 & 20 & Analista de pruebas senior 2 \\
\hline
\end{tabular}

Nota: Se ha descrito el código, código de la historia de usuario, puntos y responsable del Sprint 1. Elaboración Propia. 


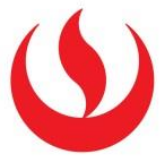

\section{Tablero Scrum}

Según lo indicado en la web de Proyectosagiles.org ("s.f."), es la lista de objetivos a completar en la iteración (Product Backlog Items). Al lado de cada objetivo se ponen las tareas necesarias para completarlo, en forma de post-its, y se van moviendo hacia la derecha para cambiarlas de estado.

Para un mejor manejo del Product Backlog se usará el siguiente tablero scrum para cada equipo diferenciado en 3 estados (To do, In Progress y Done).

Los prototipos de los tableros se realizaron mediante la herramienta SCRUMBLR (http://scrumblr.ca/) los cuales serán implementados en cada área respectiva.

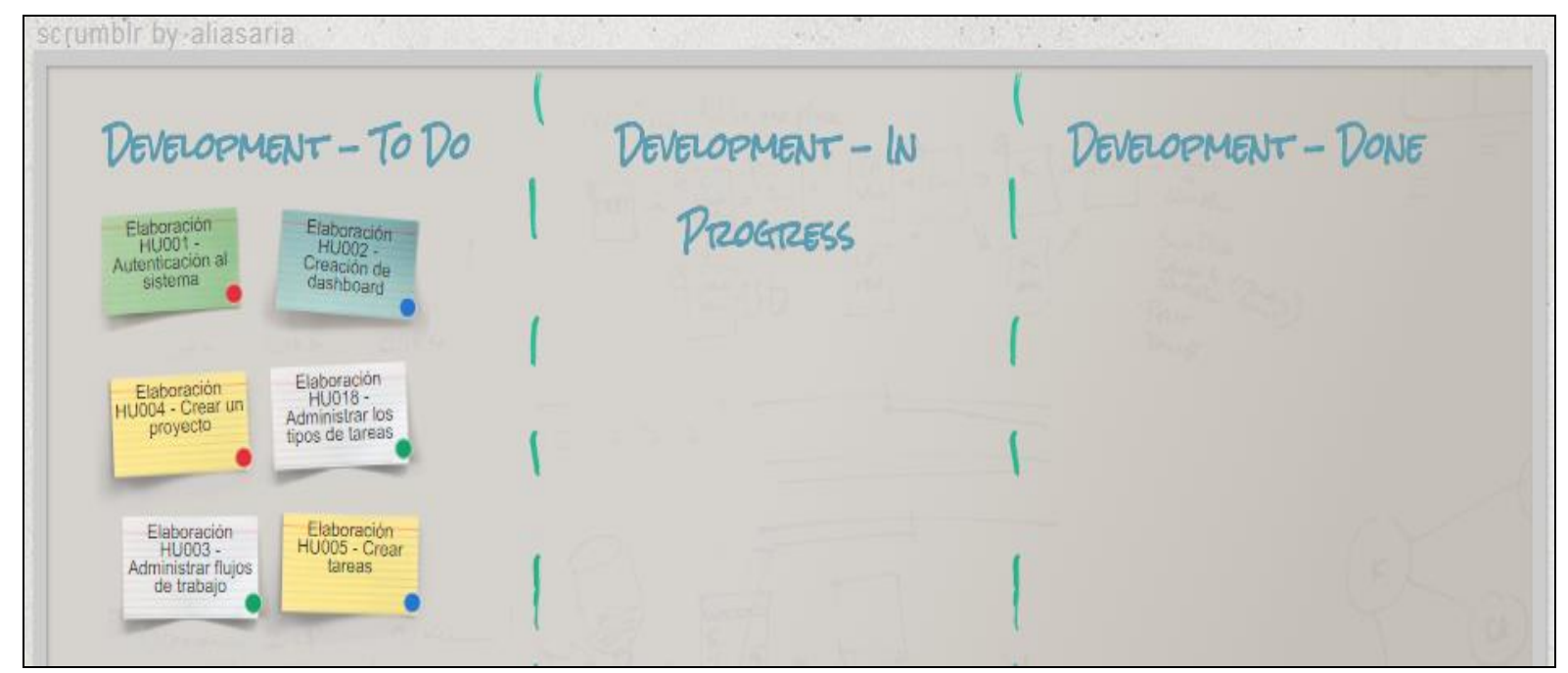

Figura 28. Prototipo del Tablero Scrum para el equipo de desarrollo. Elaboración Propia

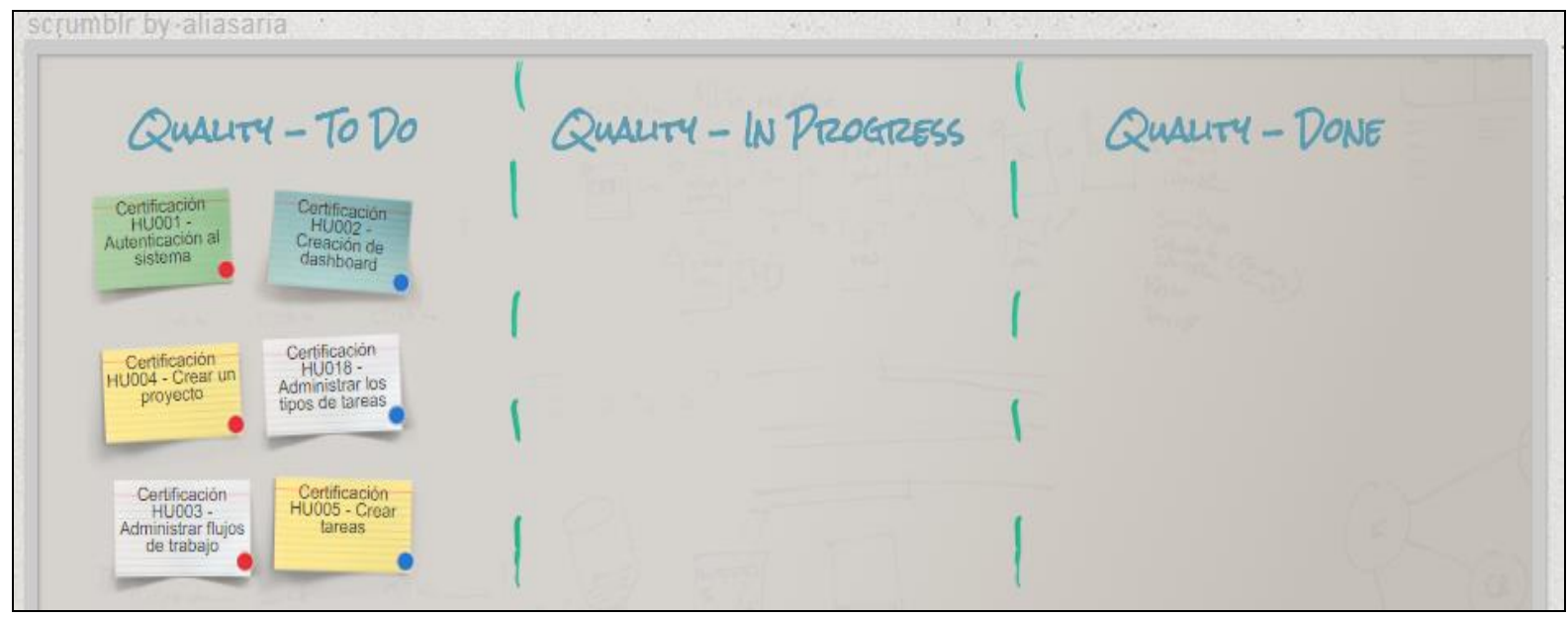

Figura 29. Prototipo del Tablero Scrum para el equipo de calidad. Elaboración Propia 


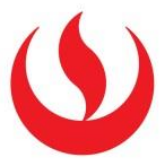

\section{Elaboración de Proyectos}

El objeto de estudio como parte de su procedimiento de iniciación de proyectos tiene un documento llamado lista de personalización, en el cual se indica los entregables que tendrá el proyecto de acuerdo al tipo, en caso el proyecto tenga una duración menor a 45 días se deberá utilizar la siguiente lista:

\begin{tabular}{|c|c|c|c|}
\hline Item & Entregas & Código & Aplica (*) \\
\hline 1 & Lista de Personalización & YGMKAAAAMMDDSSLP... & $\varpi$ \\
\hline 2 & Cronograma o SARA & YGMKAAAAMMDDSSCR... & $\varpi$ \\
\hline 3 & Documento Análisis de Requerimientos & YGMKAAAAMMDDSSAR.... & $\nabla$ \\
\hline 4 & Documento Análisis y Diseño del Producto & IproductoAD.... & $\sqrt{\bullet}$ \\
\hline 5 & Documento Interface Aplicativa & IproductolA.... & $\Gamma$ \\
\hline 6 & Acta de Reunión Comité Técnico & YGMKAAAAMMDDSSACComite... & Г \\
\hline 7 & Codigo Fuente & & $\sqrt{\downarrow}$ \\
\hline 8 & Manual de Usuario & IproductoMU... & $\Gamma$ \\
\hline 9 & Manual de Instalación & IproductoMI... & $\nabla$ \\
\hline 10 & Manual de Actualización de Versión & IproductoAV... & $\nabla$ \\
\hline 11 & $\begin{array}{l}\text { Especificación y Resultado de Casos de Pruebas en el } \\
\text { Sistema de Gestión de Pruebas }\end{array}$ & IproductoRP... & $\nabla$ \\
\hline 12 & Informe final de Certificación & IproductolC... & $\nabla$ \\
\hline 13 & Documento de Implementación & CIDdelclientelM & $\varpi$ \\
\hline 14 & Documento de Implantación & YGMKAAAAMMDDSSIP.... & $\Gamma$ \\
\hline 15 & Sistema instalado en ambiente de desarrollo del cliente & & $\sqrt{\nabla}$ \\
\hline 16 & Informe de pruebas en ambiente de desarrollo del cliente & & $\Gamma$ \\
\hline 17 & Sistema instalado en ambiente de certificación del cliente & & Г \\
\hline 18 & Bitacora de pruebas en ambiente de certificación del cliente & & $\Gamma$ \\
\hline 19 & Soporte a Certificación & & $\Gamma$ \\
\hline 20 & Acta de conformidad del cliente & YGMKAAAAMMDDSSACActaConformidad & $\nabla$ \\
\hline 21 & Acta de aceptación de pase a soporte & & $\varpi$ \\
\hline 22 & Acta de Conformidad ADC & & $\nabla$ \\
\hline
\end{tabular}

Figura 30. Lista de Personalización Proyecto Base. Adaptado de "Procedimiento de Iniciación", por El Objeto de Estudio, 2017. 


\section{(4)}

La lista de personalización que se aplicaría al proyecto tendría que referenciar los documentos de Scrum, quedaría de la siguiente manera:

\begin{tabular}{|c|c|c|c|}
\hline Item & Entregas & Código & Aplica $(*)$ \\
\hline 1 & Lista de Personalización & YGMKAAAAMMDDSSLP... & $\nabla$ \\
\hline 2 & Cronograma o Jira & YGMKAAAAMMDDSSCR... & $\varpi$ \\
\hline 3 & Documento Análisis de Requerimientos o Product Backlog & YGMKAAAAMMDDSSAR.... & $\nabla$ \\
\hline 4 & Documento Sprint Backlog & YGMKAAAAMMMDDSSSB.... & $\varpi$ \\
\hline 5 & Documento Análisis y Diseño del Producto o Historia de Usuario & IproductoAD.... & $\sqrt{\square}$ \\
\hline 6 & Documento Interface Aplicativa & IproductolA.... & $\Gamma$ \\
\hline 7 & Acta de Reunión Comité Técnico & YGMKAAAAMMMDDSSACComite... & \ulcorner \\
\hline 8 & Codigo Fuente & & $\varpi$ \\
\hline 9 & Manual de Usuario & IproductoMU... & $\Gamma$ \\
\hline 10 & Manual de Instalación & IproductoMI... & $\nabla$ \\
\hline 11 & Manual de Actualización de Versión & IproductoAV... & $\nabla$ \\
\hline 12 & $\begin{array}{l}\text { Especificación y Resultado de Casos de Pruebas en el Sistema de Gestión de } \\
\text { Pruebas }\end{array}$ & IproductoRP... & $\nabla$ \\
\hline 13 & Informe final de Certificación & IproductolC... & $\varpi$ \\
\hline 14 & Documento de Implementación & CIDdelclientelM & $\varpi$ \\
\hline 15 & Documento de Implantación & YGMKAAAAMMMDDSSIP.... & $\Gamma$ \\
\hline 16 & Sistema instalado en ambiente de desarrollo del cliente & & $\nabla$ \\
\hline 17 & Informe de pruebas en ambiente de desarrollo del cliente & & $\Gamma$ \\
\hline 18 & Sistema instalado en ambiente de certificación del cliente & & \ulcorner \\
\hline 19 & Bitacora de pruebas en ambiente de certificación del cliente & & $\Gamma$ \\
\hline 20 & Soporte a Certificación & & $\Gamma$ \\
\hline 21 & Acta de conformidad del cliente & YGMKAAAAMMMDDSSACActaConformidad & $\nabla$ \\
\hline 22 & Acta de aceptación de pase a soporte & & $\varpi$ \\
\hline 23 & Acta de Conformidad ADC & & $\varpi$ \\
\hline
\end{tabular}

Figura 31. Lista de Personalización Proyecto Scrum. Elaboración propia. 


\section{(1)}

\section{CAPÍTULO 4: PROPUESTA DEL PROYECTO}

\section{RESUMEN EJECUTIVO}

Se ha identificado que la empresa, dedicada a la consultoría de software, presenta ciertos problemas en uno de sus procesos claves, Gestión de Proyectos. Lo cuales se detallan:

- El control del cronograma de proyecto no es óptimo

- Mala gestión de los entregables para esta etapa del proyecto.

- Seguimiento de la creación/actualización de los entregables.

Sabiendo estos problemas, se desea realizar una mejora mediante la implementación del nuevo sistema JIRA que reemplazará los 3 sistemas existentes de Gestión de Proyectos para que pueda dar un mejor soporte al proceso y ayude en la solución de estos inconvenientes. Esta propuesta nace gracias al desarrollo de una Arquitectura Empresarial, basado en el marco de trabajo TOGAF, en la empresa y con la ejecución de este proyecto mediante el marco de trabajo Scrum. 


\section{(}

\section{MARCO TEÓRICO}

\section{Arquitectura Empresarial}

Según Zachman (1987), el marco teórico para el desarrollo de arquitecturas empresariales, distribuye perfectamente la arquitectura de negocio, procesos de negocio y objetivos empresariales, con las arquitecturas de aplicaciones, arquitectura de datos y arquitectura tecnológica de la empresa; cada elemento de cada arquitectura se encuentra correctamente enlazado logrando una sincronía total y con ello una excelente gestión de negocio.

Según Arango-Serna, Londoño-Salazar \& Branch-Bedoya (2015) indican que la Arquitectura Empresarial (AE) se concentra en apoyar los siguientes aspectos:

- Acompañar el desarrollo y la ejecución de la estrategia de negocio en su proceso de transformación contínuo.

- Mejorar de manera progresiva los procesos y las capacidades de negocio.

- Operacionalizar en la práctica los cambios que muestra la empresa, y que influyen toda la estructura operativa.

- Apoyar la orientación tecnológica alineado con las estrategias de negocio y examinar capacidades de innovación a partir de los recursos y capacidades existentes

\section{TOGAF}

Según The Open Group (1999 - 2018), TOGAF es un marco de trabajo que puede ser utilizado libremente por cualquier empresa que requiera desarrollar una arquitectura empresarial. Además, abarca el desarrollo de cuatro dominios de arquitecturas que vienen a ser como subconjuntos de una arquitectura empresarial, los cuales se detallan:

La arquitectura de negocios: define la estrategia comercial, el gobierno, la organización y los procesos clave de negocio. 


\section{(1)}

La arquitectura de datos: detalla la estructura de los activos de datos lógicos y físicos de una empresa y los recursos de gestión de datos.

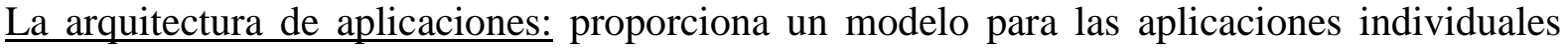
que se implementarán, sus interacciones y sus relaciones con los procesos empresariales centrales de la organización.

La arquitectura de tecnología: detalla las capacidades lógicas de software y hardware que se requieren para admitir la implementación de servicios empresariales, de datos y de aplicaciones; esto incluye infraestructura de TI, middleware, redes, etc.

\section{Método de Desarrollo de Arquitectura}

Proporciona un proceso validado y repetible para el desarrollo de arquitecturas. Incluye el establecimiento de un marco de arquitectura, el desarrollo de contenido de arquitectura, la transición y el gobierno de la realización de arquitecturas. Todas estas actividades se llevan a cabo dentro de un ciclo iterativo de definición y realización continua de arquitecturas que permite a las organizaciones transformar sus empresas de forma controlada en respuesta a los objetivos y oportunidades comerciales. Las fases dentro del ADM son las siguientes:

- Fase Preliminar

- Fase A: Visión de la Arquitectura

- Fase B: Arquitectura de Negocio

- Fase C: Arquitectura de Sistemas de Información:

- Fase D: Arquitectura Tecnológica

- Fase E: Oportunidades y Soluciones

- Fase F: Planeación de Migraciones

- Fase G: Implementación de la Gobernanza

- Fase H: Gestión de la arquitectura de cambio 


\section{(1)}

\section{Metodologías Ágiles}

Tal como indican Navarro, Fernández y Morales (2013), en la década de los noventa surgieron metodologías de desarrollo de software ligeras, que más adelante fueron llamadas ágiles, las cuales tienen el fin de reducir la probabilidad del fracaso por subvaloración de costos, tiempos y funcionalidades en los proyectos de desarrollo de software.

Las metodologías ágiles se basan en un enfoque flexible, pueden ser modificadas para que se ajusten a la realidad de cada equipo y proyecto. Cada proyecto se trabaja de manera independiente y desarrolla un subconjunto de características durante un periodo de tiempo corto, de entre dos o seis semanas. La comunicación con el cliente es constante al punto de requerir un representante de él durante el desarrollo. Los proyectos son colaborativos y se acoplan a los cambios. El cambio en los requerimientos es una característica esperada y deseada, al igual que las entregas constantes al cliente y la retroalimentación por parte de él.

\section{Metodologías ágiles representativas}

Tal como indican Navarro, Fernández y Morales (2013), las metodologías ágiles más representativas se detallan a continuación:

Scrum: Para el desarrollo ágil de software es un marco de trabajo diseñado para lograr la colaboración eficaz de equipos en proyectos, que utiliza un conjunto de reglas y artefactos y define roles que generan la estructura necesaria para su funcionamiento correcto.

Extreme Programming (XP): Es una metodología ágil centrada en guiar equipos de desarrollo de software pequeños o medianos, entre dos y diez desarrolladores, en ambientes de requerimientos imprecisos o cambiantes.

\section{Marco de Trabajo: SCRUM}

Tal como indican Navarro, Fernández \& Morales (2013), SCRUM utiliza un enfoque incremental que tiene como fundamento la teoría de control empírico de procesos. Dicha 


\section{(1)}

teoría se basa en la transparencia, inspección y adaptación; la transparencia , que avala la visibilidad en el proceso de las cosas que pueden afectar el resultado; la inspección, que ayuda a descubrir variaciones indeseables en el proceso; y la adaptación, que realiza los ajustes pertinentes para minimizar el impacto de las mismas.

En Scrum se manejan equipos los cuales son auto-gestionados, multifuncionales y trabajan en iteraciones. La entrega del producto se hace en iteraciones; cada iteración crea nueva funcionalidades o modifica las que el dueño del producto requiera.

Adicionalmente, Scrum también maneja ciertos roles y responsabilidades, donde el marco de trabajo requiere de tres pero pueden existir roles adicionales. Los roles y responsabilidades de Scrum son:

- El Scrum master

- El Producto Owner o dueño del producto

- El equipo de desarrollo

Scrum maneja 5 eventos para fomentar la colaboración y comunicación del equipo reduciendo así las reuniones extensas y los procesos restrictivos y predictivos. Los eventos de Scrum son:

- El evento principal o Sprint

- Planificación del Sprint o Sprint Planning

- Daily Scrum

- La revisión del Sprint o Sprint Review

- La Retrospectiva del Sprint o Sprint Retrospective

En Scrum existen también los artefactos, estos son subproductos de las actividades del marco de trabajo que le brindan dirección y transparencia al equipo. Los artefactos Scrum son:

- El Product Backlog

- El Sprint Backlog

- El Monitoreo de Progreso

- El Incremento 


\section{(4)}

\section{Beneficios del Marco de Trabajo SCRUM}

Tabla 103

Cuadro de Beneficios del Marco de Trabajo SCRUM

\begin{tabular}{|c|c|}
\hline Beneficio & Cómo se consiguen \\
\hline \multirow{2}{*}{$\begin{array}{l}\text { Gestión regular de las } \\
\text { expectativas del cliente }\end{array}$} & Lista de requisitos priorizada \\
\hline & $\begin{array}{l}\text { Demostración de los resultados de proyecto en cada } \\
\text { iteración }\end{array}$ \\
\hline Flexibilidad y adaptación & Replanificación en el inicio de cada iteración \\
\hline Mitigación de riesgos & Desarrollo iterativo e incremental \\
\hline \multirow[t]{6}{*}{ Productividad y calidad } & Mejora continua \\
\hline & Comunicación diaria del equipo \\
\hline & TimeBoxing \\
\hline & Estimación de esfuerzo conjunta \\
\hline & Compromiso del equipo \\
\hline & $\begin{array}{l}\text { Demostración de resultados preparados para ser } \\
\text { utilizados y velocidad sostenida }\end{array}$ \\
\hline $\begin{array}{l}\text { Alineamiento entre cliente } y \\
\text { equipo }\end{array}$ & Cliente y equipo trabajando "en equipo" \\
\hline \multirow[t]{2}{*}{ Equipo motivado } & Equipo autogestionado \\
\hline & Demostración \\
\hline
\end{tabular}

Nota: Se ha descrito el nombre y como se consigue los beneficios de Scrum. Adaptado de "Beneficios de Scrum", por Proyectosagiles.org, "s.f.".

\section{Criterios de evaluación financiera de inversión}

Como mencionan las autoras Alarcón, Ullua \& Carretero (2014), existen citeriores de evaluación financiera a usar para decidir si los proyectos se podrán aceptar o no.

En esta sección solo se considerará dos principales criterios indicando el concepto de cada uno, la forma de cálculo y criterio de decisión. 


\section{(1)}

\section{El Valor Actual Neto (VAN)}

Como concepto las autoras mencionan que el VAN "es la ganancia neta del proyecto, es decir, una contribución neta al valor de la empresa, que se da siempre y cuando los ingresos descontados son superiores a la erogación de efectivo inicial. En otras palabras, es el flujo de tesorería descontado a una tasa apropiada (costo de oportunidad), más cualquier flujo de efectivo inmediato, en el caso de una inversión en activos es negativo."

\section{Fórmula de cálculo:}

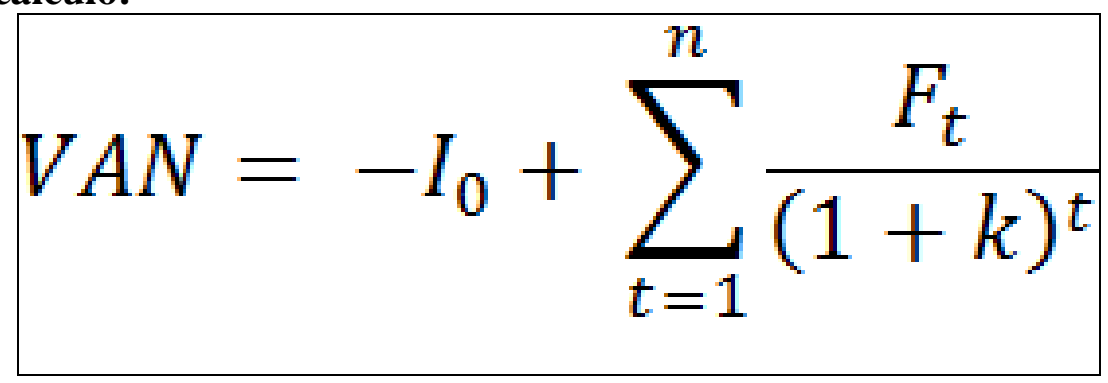

Figura 32. Formula del VAN. Adaptado de "Las Decisiones de Presupuesto de Capital: Criterios Financieros Fundamentales”, por Alarcòn, Ullua y Carretero, 2014.

Donde:

$$
\begin{aligned}
& I_{0}=\text { Inversión inicial en el año } 0 \\
& t=\text { El número de periodos de tiempo } \\
& F_{t}=\text { Flujo de dinero en cada periodo } t \\
& k=\text { Tasa de descuento }
\end{aligned}
$$

Figura 33. Variables del VAN. Adaptado de "Las Decisiones de Presupuesto de Capital: Criterios Financieros Fundamentales", por Alarcòn, Ullua y Carretero, 2014.

\section{Criterio:}

Finalmente, las autoras dicen que "si los proyectos evaluados (dos o más), son mutuamente excluyentes se acepta el de mayor VAN positivo, si todos tienen VAN negativo se rechazan. Si los proyectos son independientes se pueden aceptar todos los que tengan VAN positivo". 


\section{(1)}

\section{La Tasa Interna de Rentabilidad (TIR)}

Como concepto las autoras mencionan que el TIR es un "criterio que se usa para evaluar las propuestas de inversión mediante la aplicación de la tasa de rendimiento sobre un activo, la cual se calcula encontrando la tasa de descuento que iguala el valor actual de los flujos futuros de entrada de efectivo al costo de la inversión, o lo que es lo mismo, aquella tasa que hace cero la ecuación del VAN. Por lo que tasa es interna de la empresa (para ese proyecto), diferente al costo de oportunidad, que es del mercado. Se puede encontrar este valor de varias formas, el más usado es el de prueba y error."

\section{Fórmula de cálculo:}

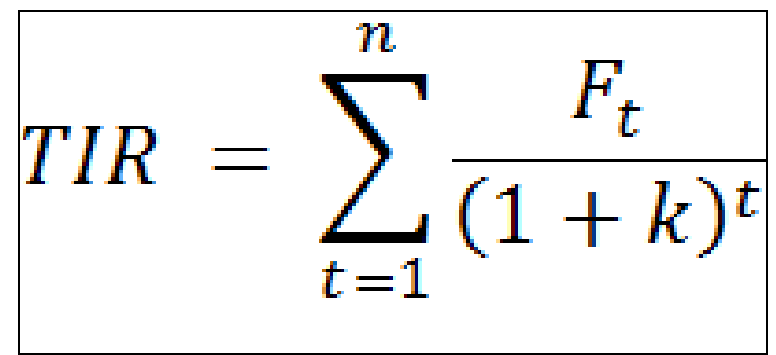

Figura 34. Formula del TIR. Adaptado de "Las Decisiones de Presupuesto de Capital: Criterios Financieros Fundamentales", por Alarcòn, Ullua \& Carretero, 2014.

Donde:

$$
\begin{aligned}
& t=\text { El número de periodos de tiempo } \\
& F_{t}=\text { Flujo de dinero en cada periodo } t \\
& k=\text { Tasa de descuento }
\end{aligned}
$$

Figura 35. Variables del TIR. Adaptado de "Las Decisiones de Presupuesto de Capital: Criterios Financieros Fundamentales”, por Alarcòn, Ullua \& Carretero, 2014.

\section{Criterio:}

Finalmente, las autoras dicen que "la TIR es un criterio alternativo y complementario al VAN, ya que además de ser otro juicio que ayuda a tomar las decisiones de inversión, conduce a la misma decisión que éste." 


\section{POSICIONAMIENTO}

Planteamiento del problema

○ MAPA DE PROCESOS Y FUNCIONES DE NEGOCIO

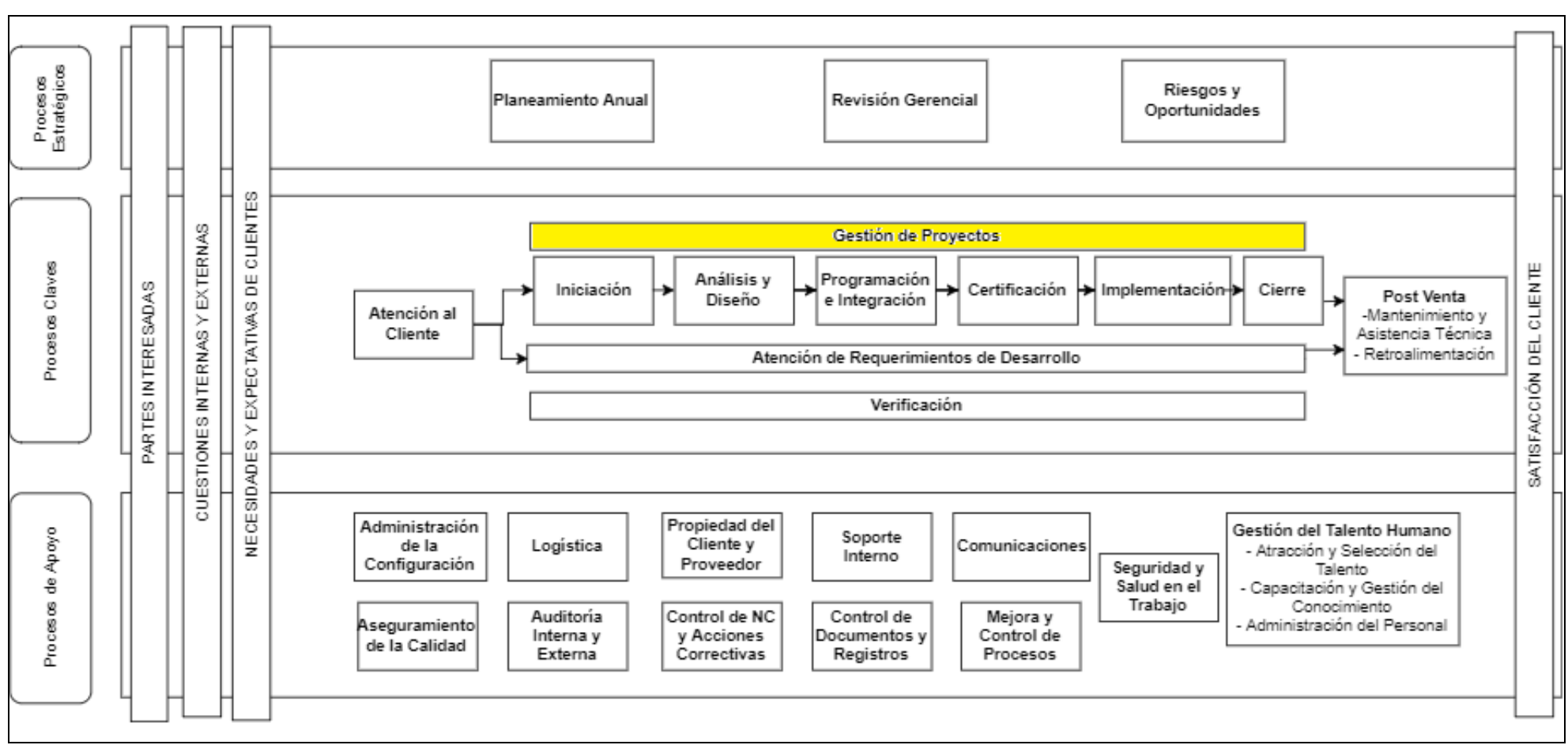

Figura 36. Mapa de procesos de la empresa. Adaptado de "Manual de calidad", por El Objeto de Estudio, 2017. 


\section{(1)}

Tabla 104

Objetivo del Proceso de Gestión de Proyectos

\begin{tabular}{|c|c|c|}
\hline $\mathbf{N}^{\mathbf{o}}$ & $\begin{array}{l}\text { PROCESO } \\
\text { CLAVE }\end{array}$ & OBJETIVO DEL PROCESO \\
\hline 1 & $\begin{array}{l}\text { Gestión } \\
\text { Proyectos }\end{array}$ & 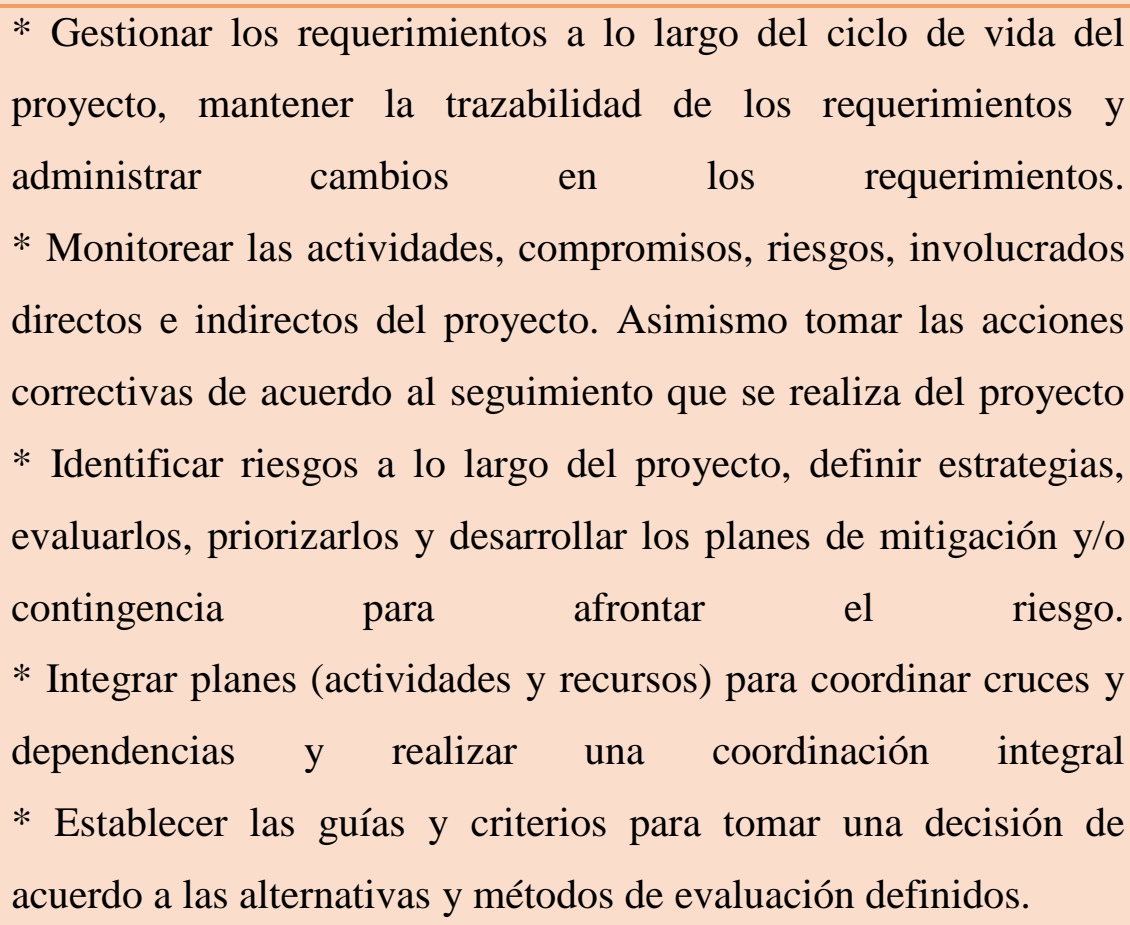 \\
\hline
\end{tabular}

Nota: Se ha descrito los objetivos del proceso de Gestión de Proyectos. Adaptado de "Manual de calidad", por El Objeto de Estudio, 2017.

Tabla 105

Cuadro de problemas del proceso de Gestión de Proyectos

\begin{tabular}{|l|l|l|}
\hline PROCESO & PROBLEMAS & CAUSAS \\
\hline GESTIÓN & El control manual del & El control del proyecto se hace \\
DE & cronograma del proyecto no es & de manera manual obteniendo la \\
PROYECTOS & óptimo & $\begin{array}{l}\text { información del archivo Project, } \\
\text { el cual tiende a ser actualizado } \\
\text { de manera repetitiva y por cada } \\
\end{array}$ \\
& & $\begin{array}{l}\text { cambio que se quiera realizar se } \\
\text { tiene que exportar lo que existe } \\
\end{array}$ \\
& & en el SARA, realizar el cambio e \\
& & importar nuevamente en el \\
\hline
\end{tabular}




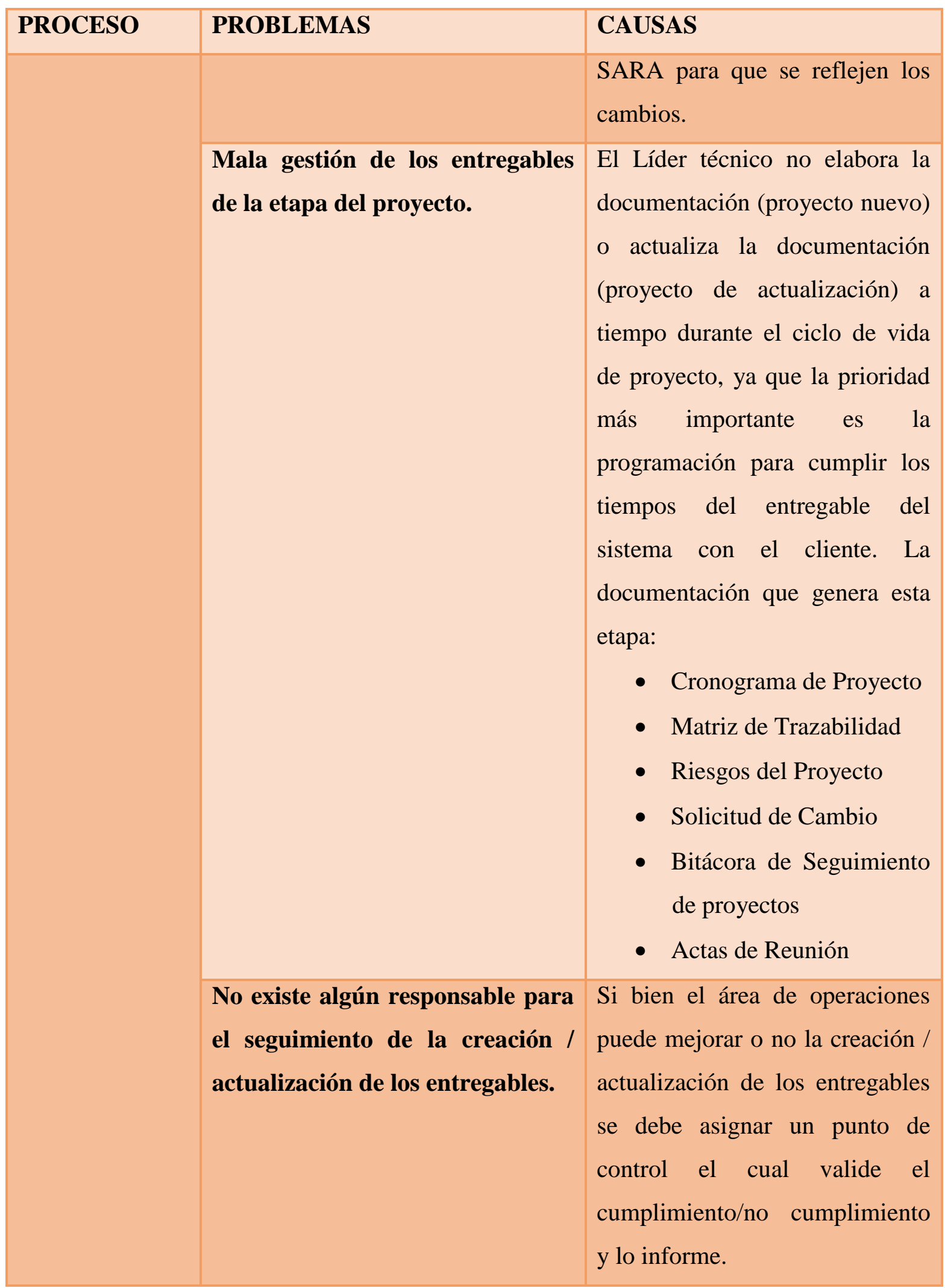

Nota: Se ha descrito el nombre y objetivo de los indicadores de éxito. Adaptado de "Indicadores de la Organización”, por El Objeto de Estudio, 2017. 


\section{(}

Tabla 106

Sistemas vs Problemas

\begin{tabular}{|c|c|}
\hline SISTEMAS & PROBLEMAS \\
\hline Sistema SARA & $\begin{array}{l}\text { Lenguaje de programación obsoleto } \\
\text { El sistema SARA es más utilizado como un repositorio de } \\
\text { datos con el cual se comunican los otros subsistemas } \\
\text { existentes de la empresa. No se realizan mejoras o nuevos } \\
\text { módulos ya que la Gerencia no desea invertir en tecnología } \\
\text { antigua. }\end{array}$ \\
\hline $\begin{array}{l}\text { Sistema } \\
\text { IMPORT/EXPORT }\end{array}$ & $\begin{array}{l}\text { Subsistemas aislado con otros subsistemas } \\
\text { El sistema import/export ayuda a los líderes técnicos a bajar la } \\
\text { información que se encuentra en el SARA, actualizar el } \\
\text { cronograma manualmente y subirlo nuevamente al SARA. } \\
\text { Solo comparte información con el Sistema SARA, actualiza el }\end{array}$ \\
\hline $\begin{array}{l}\text { Sistema Procesador de } \\
\text { Cronogramas }\end{array}$ & $\begin{array}{l}\text { archivo Project el cual necesita el Sistema Procesador de } \\
\text { Cronogramas pero no tiene relación con él. } \\
\text { El sistema procesador de Cronogramas procesa el cronograma } \\
\text { actualizado que se importó (IMPORT/EXPORT) al SARA y } \\
\text { luego de que hayan llenado sus horas correspondientes todo el } \\
\text { equipo de trabajo. Solo comparte información con el Sistema } \\
\text { SARA }\end{array}$ \\
\hline
\end{tabular}

Nota: Se ha identificado los problemas existentes en los sistemas del proceso de gestión de proyectos. Adaptado de "Procedimiento de Gestión de Proyecto", por El Objeto de Estudio, 2017. 


\section{(}

\section{Objetivos}

\section{Objetivo General}

- Elaborar una propuesta de arquitectura empresarial enfocada en la mejora y optimización del proceso de Gestión de Proyectos y sus herramientas del Objeto de Estudio para un mejor desempeño de la organización.

\section{Objetivos Específicos}

- Identificar las posibles soluciones en el proceso de Gestión de Proyectos en base a una arquitectura empresarial

- Proponer la implementación de un nuevo software que de soporte al proceso de Gestión de Proyectos bajo el marco de trabajo Scrum.

- Proponer la actualización del procedimiento de Gestión de Proyectos en base al nuevo software.

\section{Indicadores de éxito}

Tabla 107

Cuadro de Indicadores de éxito del proyecto

\begin{tabular}{|c|c|c|}
\hline $\begin{array}{l}\text { Nombre del } \\
\text { Indicador }\end{array}$ & Descripción del indicador & Objetivos Esp \\
\hline $\begin{array}{lr}\text { Satisfacción } & \text { del } \\
\text { uso del } & \text { nuevo } \\
\text { aplicativo } & \text { de } \\
\text { gestión } & \text { de } \\
\text { proyectos } & \end{array}$ & $\begin{array}{l}\text { - En base a una encuesta se } \\
\text { podrá validar si los } \\
\text { trabajadores sienten que el } \\
\text { nuevo aplicativo es más fácil } \\
\text { de usar. }\end{array}$ & $\begin{array}{l}\text { - Proponer la implementación } \\
\text { de un nuevo software que de } \\
\text { soporte al proceso de Gestión } \\
\text { de Proyectos bajo el marco de } \\
\text { trabajo Scrum. }\end{array}$ \\
\hline $\begin{array}{l}\text { Cumplimiento de } \\
\text { los requisitos } \\
\text { funcionales. }\end{array}$ & $\begin{array}{l}\text { - En base a un check list se } \\
\text { deberá validar que el } \\
\text { aplicativo cumpla con todos } \\
\text { los requisitos funcionales } \\
\text { existentes para asegurar la }\end{array}$ & $\begin{array}{l}\text { - Proponer la implementación } \\
\text { de un nuevo software que de } \\
\text { soporte al proceso de Gestión } \\
\text { de Proyectos bajo el marco de } \\
\text { trabajo Scrum. }\end{array}$ \\
\hline
\end{tabular}




\begin{tabular}{|c|c|c|}
\hline $\begin{array}{l}\text { Nombre del } \\
\text { Indicador }\end{array}$ & Descripción del indicador & Objetivos Específicos \\
\hline & calidad del entreoable & \\
\hline $\begin{array}{l}\text { No conformidades } \\
\text { cerradas }\end{array}$ & $\begin{array}{l}\text { - En base al Sistema de Gestión } \\
\text { de No conformidades se podrá } \\
\text { obtener los datos para el } \\
\text { indicador. Dicho indicador } \\
\text { consiste en sacar el número de } \\
\text { no conformidades cerradas en } \\
\text { el proceso de gestión de } \\
\text { proyectos. } \\
\text { La fórmula será: } \\
\text { (Número de no conformidades } \\
\text { cerradas de gestión de proyectos / } \\
\text { Número total de no } \\
\text { conformidades de gestión de } \\
\text { proyectos) * } 100\end{array}$ & $\begin{array}{l}\text { - Proponer la implementación } \\
\text { de un nuevo software que de } \\
\text { soporte al proceso de Gestión } \\
\text { de Proyectos bajo el marco de } \\
\text { trabajo Scrum. }\end{array}$ \\
\hline $\begin{array}{lr}\text { Optimización } & \text { de } \\
\text { tiempo } & \text { para } \\
\text { gestión } & \text { de } \\
\text { proyectos } & \text { por } \\
\text { semana } & \end{array}$ & $\begin{array}{l}\text { - En base a una encuesta se } \\
\text { recopiló el tiempo empleado } \\
\text { en la utilización de las } \\
\text { herramientas de gestión de } \\
\text { proyectos. } \\
\text { La fórmula será: } \\
\text { (Número de horas empleadas a la } \\
\text { semana por jornada laboral en el } \\
\text { proceso de gestión de proyectos / } \\
\text { Número total de horas a la semana } \\
\text { por jornada laboral en el proceso } \\
\text { de gestión de proyectos) * } 100\end{array}$ & $\begin{array}{l}\text { - Identificar las posibles } \\
\text { soluciones en el proceso de } \\
\text { Gestión de Proyectos en base } \\
\text { a una arquitectura empresarial } \\
\text { - Proponer la implementación } \\
\text { de un nuevo software que de } \\
\text { soporte al proceso de Gestión } \\
\text { de Proyectos bajo el marco de } \\
\text { trabajo Scrum. } \\
\text { Proponer la actualización del } \\
\text { procedimiento de Gestión de } \\
\text { Proyectos en base al nuevo } \\
\text { software. }\end{array}$ \\
\hline
\end{tabular}




\section{(1)}

Nota: Se ha descrito el nombre y objetivo de los indicadores de éxito. Elaboración propia.

\section{Alcance del proyecto}

El presente proyecto tiene como alcance realizar la identificación de los posibles proyectos que se pueden implementar basándose en un análisis de brechas mediante una arquitectura empresarial para el proceso Gestión de Proyectos. Mediante este portafolio se propondrá un proyecto de software a realizar y a la par la actualización del procedimiento con el fin de mantener alineado la documentación, basado en el modelo CMMI y la certificación de calidad ISO 9001:2008 que cuenta la empresa.

\section{Restricciones}

- Todo proyecto interno a ejecutarse tiene como tiempo promedio el plazo de un año.

- Todo proyecto en curso debe ser controlado de alguna manera generando informes de estado, sobre tiempos, riesgos, cambios y otros.

- Todo proyecto deberá manejar una bitácora de seguimiento para conocer todos los eventos surgidos.

- La información sobre los procesos, proyectos y fuentes de programas que cuenta la empresa es reguardada ejecutando la copia de seguridad semanal.

- Se cuenta actualmente con el tercer nivel de certificación del modelo CMMI y con certificación internacional de calidad ISO 9001:2008.

- Se maneja un presupuesto para desarrollo de proyectos por el monto de 500 mil nuevos soles (S/. 500,000.00)

\section{Impacto en la organización}

Los presentes proyectos impactarán en los siguientes términos:

Costo: Los proyectos tendrán un costo de 210 mil 508 soles (S/. 210, 508.00)

\begin{tabular}{|r|l|r|}
\hline Cantidad & Recurso & \multicolumn{1}{l|}{ Costo } \\
\hline 2 & Servidores y licencias & $120,000.00$ \\
\hline 5 & Recurso de Arquitectura Empresarial & $28,500.00$ \\
\hline 9 & Recursos Agiles & $47,008.00$ \\
\hline 1 & Modelo de base de datos & $15,000.00$ \\
\hline \multicolumn{2}{|c|}{ TOTAL } & $210,508.00$ \\
\hline
\end{tabular}




\section{(1)}

Figura 37. Costos del proyecto. Elaboración Propia

Calidad: Se deberá respetar los estándares que maneja la empresa para los proyectos, los cuales son:

- Tercer nivel de certificación del modelo CMMI.

- Certificación internacional de calidad ISO 9001:2008.

Proceso: Los proyectos deberán cumplir con los entregables definidos por la empresa. A pesar de que se está utilizando para uno de los proyectos el framework Scrum debe convivir con lo que actualmente tiene la empresa. Para el proyecto de implementación del software Jira será:

○ Lista de Personalización

- Cronograma o Jira

○ Documento Análisis de Requerimientos o Product Backlog

○ Documento Sprint Backlog

○ Documento Análisis y Diseño del Producto o Historia de Usuario

○ Código Fuente

○ Manual de Instalación / Manual de Actualización de Versión

- Especificación y Resultado de Casos de Pruebas en el Sistema de Gestión de Pruebas

- Informe final de Certificación

○ Documento de Implementación

- Sistema instalado en ambiente de desarrollo del cliente

- Acta de conformidad del cliente

- Acta de aceptación de pase a soporte

- Acta de Conformidad ADC

Para el proyecto de Mejora del procedimiento de Gestión de Proyectos serán:

- Instrucción de elaboración de la plantilla de riesgos

○ Instrucción de actualización de cronograma

○ Instrucción de criterios básicos para generar una solicitud de cambio

- Procedimiento de Gestión de Proyecto

○ Procedimiento de Gestión de Requerimientos

○ Procedimiento de Gestión de Riesgos 


\section{(1)}

Tiempo: Los proyectos tienen una duración de aproximadamente 2 meses, ya que se realizaran en forma paralela.

Organización: Los proyectos a implementar apoyaran al logro de 3 de los objetivos estratégicos planteados por la organización, los cuales son:

- Objetivo Estratégico 1: Contar con un índice de Satisfacción Global por Cliente en un 82\%. (Servicio integral: Soporte, Actividades, Proyectos).

- Objetivo Estratégico 3: Crear por lo menos 2 nuevos productos competitivos durante el año, y actualizar 2 de los actuales.

- Objetivo Estratégico 5: Incrementar en un 10\% la productividad mediante el mejoramiento de nuestros procesos (menos errores y velocidad de ejecución).

Empleados: Los proyectos a implementar una vez terminados serán expuestos a todo el personal de operaciones mediante una capacitación para que estén informados sobre los cambios que conlleva dicha implementación. Se realizará para el uso de la herramienta como para los cambios en la documentación.

\section{ORGANIZACIÓN DEL PROYECTO}

\section{Equipo del Proyecto}

Tabla 108

Cuadro del Equipo del Proyecto

\begin{tabular}{|c|c|c|}
\hline Rol & Miembro (Cantidad) & Responsabilidades \\
\hline $\begin{array}{l}\text { Arquitecto } \\
\text { Empresarial }\end{array}$ & - Analista de Sistemas Sr. (1) & $\begin{array}{l}\text { - Definir en base al sistema como } \\
\text { se atenderán las necesidades } \\
\text { - Explicar cómo se solucionarán } \\
\text { los problemas }\end{array}$ \\
\hline $\begin{array}{l}\text { Arquitecto } \\
\text { Negocio }\end{array}$ & - Líder Técnico (1) & $\begin{array}{l}\text { - Definir las necesidades del } \\
\text { proceso } \\
\text { - Identificar los problemas a }\end{array}$ \\
\hline
\end{tabular}




\section{(1)}

\begin{tabular}{|c|c|c|}
\hline Rol & Miembro (Cantidad) & Responsabilidades \\
\hline & & $\begin{array}{l}\text { solucionar } \\
\text { - Mejoras en el proceso }\end{array}$ \\
\hline $\begin{array}{l}\text { Arquitecto } \\
\text { Infraestructura }\end{array}$ & - Analista de Soporte Sr. (1) & $\begin{array}{l}\text { - Ambientar los servidores } \\
\text { - Diseñar las nuevas conexiones }\end{array}$ \\
\hline $\begin{array}{l}\text { Arquitecto } \\
\text { Información }\end{array}$ & $\begin{array}{l}\text { - Analista de Base de Datos } \\
\text { (1) }\end{array}$ & $\begin{array}{l}\text { - Validar el modelo de datos } \\
\text { propuesto } \\
\text { - Montar la nueva base de datos }\end{array}$ \\
\hline $\begin{array}{l}\text { Arquitecto } \\
\text { Aplicaciones }\end{array}$ & - Arquitecto de Sistemas (1) & $\begin{array}{l}\text { - Generación de colas y clusters } \\
\text { para el sistema } \\
\text { - Revisión de los logs del sistema }\end{array}$ \\
\hline Producto Owner & - Líder Técnico (1) & $\begin{array}{l}\text { - } \quad \text { Elaborar el product backlog } \\
\text { - } \quad \text { Priorizar necesidades } \\
\text { - } \quad \text { Explicar sus necesidades }\end{array}$ \\
\hline $\begin{array}{l}\text { Scrum Master } \\
\text { (Proveedor) }\end{array}$ & - Analista de Sistemas Sr. (1) & $\begin{array}{l}\text { - Coordinar con el equipo de } \\
\text { desarrollo } \\
\text { - Apoyo al equipo de desarrollo }\end{array}$ \\
\hline Scrum Master & - Analista de Sistemas Sr. (1) & $\begin{array}{l}\text { - Coordinar con el equipo de } \\
\text { calidad } \\
\text { - Apoyo al equipo de calidad }\end{array}$ \\
\hline $\begin{array}{l}\text { Equipo Desarrollo } \\
\text { (Proveedor) }\end{array}$ & $\begin{array}{l}\text { - Analista de base de datos } \\
\text { MySQL (1) } \\
\text { - Analista programador Sr. } \\
\text { front end (2) }\end{array}$ & $\begin{array}{l}\text { - Desarrollar las necesidades del } \\
\text { cliente } \\
\text { - Participar en las reuniones } \\
\text { - Entender las necesidades del } \\
\text { cliente }\end{array}$ \\
\hline Equipo Calidad & 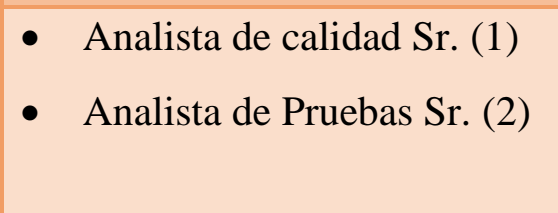 & $\begin{array}{l}\text { - Validar y verificar los } \\
\text { entregables } \\
\text { - Diseñar y ejecutar las pruebas }\end{array}$ \\
\hline
\end{tabular}

Nota: Se ha descrito el rol, miembros y responsabilidades del equipo de proyecto. Adaptado de "Manual de funciones y perfiles de puestos”, por El Objeto de Estudio, 2017. 


\section{$\boldsymbol{1}$}

\section{Stakeholders}

Tabla 109

Cuadro de Stakeholders

\begin{tabular}{|c|c|c|}
\hline Interesado & Necesidades & Entregables \\
\hline Gerente General & $\begin{array}{l}\text { Mejora en la gestión de } \\
\text { proyectos para nuevos clientes }\end{array}$ & $\begin{array}{l}\text { Informes resultantes de la gestión } \\
\text { de proyectos basado en el nuevo } \\
\text { sistema JIRA, con el fin de conocer } \\
\text { avances, retrasos entre otros. }\end{array}$ \\
\hline $\begin{array}{l}\text { Gerente de } \\
\text { Operaciones }\end{array}$ & $\begin{array}{l}\text { Mejor desempeño en el área de } \\
\text { operaciones }\end{array}$ & $\begin{array}{l}\text { Presentación del nuevo sistema } \\
\text { JIRA en producción }\end{array}$ \\
\hline $\begin{array}{l}\text { Gerente de } \\
\text { Investigación, } \\
\text { Desarrollo e } \\
\text { Innovación }\end{array}$ & $\begin{array}{l}\text { Aplicación de nuevas } \\
\text { herramientas para el soporte de } \\
\text { negocio } \\
\text { Menor no conformidades en sus } \\
\text { procesos }\end{array}$ & $\begin{array}{l}\text { Presentación del nuevo sistema } \\
\text { JIRA en producción } \\
\text { Documentación del estudio } \\
\text { realizado para la propuesta. }\end{array}$ \\
\hline
\end{tabular}

Nota: Se ha descrito el rol, necesidades y entregables de los interesados del proyecto. Adaptado de "Manual de funciones y perfiles de puestos”, por El Objeto de Estudio, 2017.

\section{Recursos requeridos}

Tabla 110

Cuadro de Recursos Requeridos

\begin{tabular}{|c|c|c|c|}
\hline Recurso & Cantidad & Características & Motivo \\
\hline $\begin{array}{l}\text { Oficinas } \\
\text { amobladas }\end{array}$ & 3 & $\begin{array}{l}\text { - Tamaño de } 7 \text { metros cuadrados } \\
\text { - Cuatro escritorios y cuatro } \\
\text { sillas por oficina } \\
\text { - Cada escritorio tendrá } 2 \text { puntos } \\
\text { de red y } 2 \text { toma corrientes. } \\
\text { - } 3 \text { anexos uno para cada oficina } \\
\text { - } 2 \text { pizarras tipo mural } 2 \text { x } 1 \\
\text { metros. }\end{array}$ & $\begin{array}{l}\text { Se tendrá una oficina } \\
\text { para el equipo de } \\
\text { desarrollo, el equipo de } \\
\text { calidad y una para el } \\
\text { producto owner donde } \\
\text { también servirá como } \\
\text { sala de reunión cuando } \\
\text { vayan los equipos. }\end{array}$ \\
\hline
\end{tabular}




\section{(4)}

\begin{tabular}{|c|c|c|c|}
\hline Recurso & Cantidad & Características & Motivo \\
\hline & & - Un proyector con cable HDMI. & \\
\hline Laptops & 9 & $\begin{array}{l}\text { - Procesador core i7 Séptima } \\
\text { generación. } \\
\text { - Tamaño de } 15 \text { pulgadas. } \\
\text { - } 3 \text { laptops con } 16 \mathrm{~GB} \text { de ram y } 6 \\
\text { con } 8 \mathrm{~GB} \text { de ram. } \\
\text { - } 9 \text { mouses. }\end{array}$ & $\begin{array}{l}\text { Se les dará a las personas } \\
\text { que realizarán la } \\
\text { ejecución del proyecto. } \\
\text { Los otros integrantes } \\
\text { mantendrán sus mismos } \\
\text { equipos ya asignados. }\end{array}$ \\
\hline Servidores & 2 & $\begin{array}{l}\text { - } 2 \text { Procesadores de } 2.53 \mathrm{Ghz} \\
\text { - Disco duro de } 10 \mathrm{~T} \mathrm{de} \\
\text { almacenamiento } \\
\text { - Memoria de } 32 \mathrm{~GB} \text { de ram }\end{array}$ & $\begin{array}{l}\text { En un servidor se } \\
\text { montará la nueva base de } \\
\text { datos, en el otro la } \\
\text { aplicación }\end{array}$ \\
\hline $\begin{array}{l}\text { Licencias } \\
\text { JIRA }\end{array}$ & 100 & $\begin{array}{l}\text { - Control total del entorno } \\
\text { - Vigencia de un año }\end{array}$ & $\begin{array}{l}\text { Para el acceso a la } \\
\text { aplicación por cada } \\
\text { recurso de la empresa. }\end{array}$ \\
\hline $\begin{array}{l}\text { CentOs } \\
\text { Linux }\end{array}$ & 2 & - Software versión 7.2 & \begin{tabular}{llr} 
Serán los & \multicolumn{2}{r}{ sistemas } \\
operativos & de & los \\
servidores & &
\end{tabular} \\
\hline $\begin{array}{l}\text { Apache } \\
\text { Tomcat }\end{array}$ & 1 & - Software versión 8.0 & $\begin{array}{l}\text { Sera el motor de } \\
\text { aplicaciones para montar } \\
\text { la aplicación }\end{array}$ \\
\hline MySQL & 1 & - Software versión 5.2 & $\begin{array}{l}\text { Será el motor de base de } \\
\text { datos donde se montará el } \\
\text { modelo de datos }\end{array}$ \\
\hline
\end{tabular}

Nota: Se ha descrito la cantidad, características y el motivo de los recursos que se van a utilizar. Adaptado de “Manual de Configuración”, por El Objeto de Estudio, 2017. 


\section{(1)}

Fases e hitos del proyecto

Tabla 111

Cuadro de Fases e hitos del proyecto de Mejora del procedimiento de Gestión de Proyectos

\begin{tabular}{|c|c|c|c|}
\hline $\begin{array}{l}\text { Fase del } \\
\text { Proyecto }\end{array}$ & Hito del Proyecto & $\begin{array}{l}\text { Fecha } \\
\text { Estimada }\end{array}$ & Entregables incluidos \\
\hline Iniciación & $\begin{array}{l}\text { - Solicitar los } \\
\text { documentos del } \\
\text { proceso de Gestión de } \\
\text { Proyectos para su } \\
\text { actualización. }\end{array}$ & $06 / 08 / 2018$ & $\begin{array}{l}\text { - Instrucción de elaboración } \\
\text { - } \text { Instrucción de actualización } \\
\text { de cronograma } \\
\text { - Instrucción de criterios } \\
\text { básicos para generar una } \\
\text { solicitud de cambio } \\
\text { - Procedimiento de Gestión de } \\
\text { Proyecto } \\
\text { - Procedimiento de Gestión de } \\
\text { Requerimientos } \\
\text { Procedimiento de Gestión de } \\
\text { Riesgos }\end{array}$ \\
\hline \multirow[t]{4}{*}{ Ejecución } & $\begin{array}{l}\text { - Actualización } \\
\text { Instrucción }\end{array}$ & $07 / 08 / 2018$ & $\begin{array}{l}\text { - Instrucción de elaboración } \\
\text { de la plantilla de riesgos }\end{array}$ \\
\hline & $\begin{array}{l}\text { Actualización del } \\
\text { Procedimiento de } \\
\text { Gestión de Riesgos }\end{array}$ & $09 / 08 / 2018$ & $\begin{array}{l}\text { - Procedimiento de Gestión de } \\
\text { Riesgos }\end{array}$ \\
\hline & $\begin{array}{ll}\text { - Actualización } & \text { de la } \\
\text { Instrucción } & \text { de } \\
\text { actualización } & \text { de } \\
\text { cronograma } & \end{array}$ & $14 / 08 / 2018$ & $\begin{array}{l}\text { - Instrucción de actualización } \\
\text { de cronograma }\end{array}$ \\
\hline & - Actualización de la & $16 / 08 / 2018$ & - Instrucción \\
\hline
\end{tabular}




\section{(1)}

\begin{tabular}{|c|c|c|c|}
\hline \multirow[t]{4}{*}{$\begin{array}{l}\text { Fase del } \\
\text { Proyecto }\end{array}$} & Hito del Proyecto & $\begin{array}{l}\text { Fecha } \\
\text { Estimada }\end{array}$ & Entregables incluidos \\
\hline & $\begin{array}{l}\text { Instrucción de } \\
\text { criterios básicos para } \\
\text { generar una solicitud } \\
\text { de cambio }\end{array}$ & & $\begin{array}{l}\text { básicos para generar una } \\
\text { solicitud de cambio }\end{array}$ \\
\hline & $\begin{array}{l}\text { - Actualización del } \\
\text { Procedimiento de } \\
\text { Gestión de Proyecto }\end{array}$ & $20 / 08 / 2018$ & $\begin{array}{l}\text { - Procedimiento de Gestión de } \\
\text { Proyecto }\end{array}$ \\
\hline & $\begin{array}{ll}\text { - Actualización } & \text { del } \\
\text { Procedimiento de } & \text { de } \\
\text { Gestión } & \text { de } \\
\text { Requerimientos } & \end{array}$ & $23 / 08 / 2018$ & $\begin{array}{l}\text { - Procedimiento de Gestión de } \\
\text { Requerimientos }\end{array}$ \\
\hline \multirow[t]{4}{*}{ Verificación } & $\begin{array}{l}\text { - Verificación de la } \\
\text { Instrucción } \\
\text { elaboración de la } \\
\text { plantilla de riesgos } \\
\text { del proyecto. }\end{array}$ & 09/08/2018 & $\begin{array}{l}\text { - Instrucción de elaboración } \\
\text { de la plantilla de riesgos } \\
\text { verificado }\end{array}$ \\
\hline & $\begin{array}{l}\text { - Verificación del } \\
\text { Procedimiento de } \\
\text { Gestión de Riesgos }\end{array}$ & $14 / 08 / 2018$ & $\begin{array}{l}\text { - Procedimiento de Gestión de } \\
\text { Riesgos verificado }\end{array}$ \\
\hline & $\begin{array}{lll}\text { - } & \text { Verificación de } & \text { la } \\
\text { Instrucción } & & \text { de } \\
\text { actualización } & & \text { de } \\
\text { cronograma } & \end{array}$ & $16 / 08 / 2018$ & $\begin{array}{l}\text { - Instrucción de actualización } \\
\text { de cronograma verificado }\end{array}$ \\
\hline & $\begin{array}{l}\text { - Verificación de la } \\
\text { Instrucción de } \\
\text { criterios básicos para } \\
\text { generar una solicitud } \\
\text { de cambio }\end{array}$ & $20 / 08 / 2018$ & $\begin{array}{l}\text { - Instrucción de criterios } \\
\text { básicos para generar una } \\
\text { solicitud de cambio } \\
\text { verificado }\end{array}$ \\
\hline
\end{tabular}




\section{(1)}

\begin{tabular}{|c|c|c|c|}
\hline $\begin{array}{l}\text { Fase del } \\
\text { Proyecto }\end{array}$ & Hito del Proyecto & $\begin{array}{l}\text { Fecha } \\
\text { Estimada }\end{array}$ & Entregables incluidos \\
\hline & $\begin{array}{l}\text { Verificación del } \\
\text { Procedimiento de } \\
\text { Gestión de Proyecto }\end{array}$ & $23 / 08 / 2018$ & $\begin{array}{l}\text { Procedimiento de Gestión de } \\
\text { Proyecto verificado }\end{array}$ \\
\hline & $\begin{array}{ll}\text { - Verificación } & \text { del } \\
\text { Procedimiento } & \text { de } \\
\text { Gestión } & \text { de } \\
\text { Requerimientos } & \end{array}$ & $27 / 08 / 2018$ & $\begin{array}{l}\text { - Procedimiento de Gestión de } \\
\text { Requerimientos verificado }\end{array}$ \\
\hline Cierre & $\begin{array}{l}\text { - Ingreso al repositorio } \\
\text { de los documentos del } \\
\text { proceso de Gestión de } \\
\text { Proyectos } \\
\text { actualizados. }\end{array}$ & $29 / 08 / 2018$ & $\begin{array}{l}\text { - Instrucción de elaboración } \\
\text { de la plantilla de riesgos } \\
\text { actualizado } \\
\text { - Instrucción de actualización } \\
\text { de cronograma actualizado } \\
\text { - Instrucción de criterios } \\
\text { básicos para generar una } \\
\text { solicitud de cambio } \\
\text { actualizado } \\
\text { Procedimiento de Gestión de } \\
\text { Proyecto actualizado } \\
\text { Procedimiento de Gestión de } \\
\text { Requerimientos actualizado } \\
\text { Procedimiento de Gestión de } \\
\text { Riesgos actualizado }\end{array}$ \\
\hline Capacitación & $\begin{array}{l}\text { - Capacitación sobre } \\
\text { los cambios del } \\
\text { proceso de gestión de } \\
\text { proyectos a todo el } \\
\text { personal de } \\
\text { operaciones. }\end{array}$ & 03/09/2018 & $\begin{array}{l}\text { - Presentación de la } \\
\text { capacitación del proceso de } \\
\text { gestión de proyectos }\end{array}$ \\
\hline
\end{tabular}

Nota: Se ha descrito las fases, hitos, fechas estimadas y entregables del proyecto. Elaboración propia. 


\section{(1)}

Tabla 112

Cuadro de Fases e hitos del proyecto de Implementación Software JIRA

\begin{tabular}{|c|c|c|c|}
\hline $\begin{array}{l}\text { Fase del } \\
\text { Proyecto }\end{array}$ & Hito del Proyecto & $\begin{array}{l}\text { Fecha } \\
\text { Estimada }\end{array}$ & Entregables incluidos \\
\hline \multirow[t]{3}{*}{$\begin{array}{l}\text { Análisis de la } \\
\text { situación }\end{array}$} & $\begin{array}{l}\text { - Presentación de la } \\
\text { Petición de trabajo de } \\
\text { arquitectura }\end{array}$ & $06 / 04 / 2018$ & $\begin{array}{l}\text { - Petición de trabajo de } \\
\text { arquitectura }\end{array}$ \\
\hline & 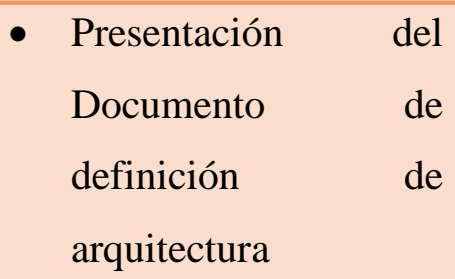 & $20 / 04 / 2018$ & $\begin{array}{l}\text { - Documento de definición } \\
\text { de arquitectura }\end{array}$ \\
\hline & $\begin{array}{l}\text { - Presentación del Plan } \\
\text { de implementación de } \\
\text { la migración }\end{array}$ & $04 / 05 / 2018$ & $\begin{array}{l}\text { - Plan de implementación de } \\
\text { la migración }\end{array}$ \\
\hline \multirow[t]{4}{*}{ Iniciación } & $\begin{array}{l}\text { - Entrega de la lista de } \\
\text { personalización }\end{array}$ & $02 / 07 / 2018$ & - Lista de personalización \\
\hline & $\begin{array}{l}\text { - Entrega de } \\
\text { cronograma }\end{array}$ & $03 / 07 / 2018$ & - Cronograma \\
\hline & $\begin{array}{l}\text { - Entrega del Análisis } \\
\text { de Requerimientos o } \\
\text { ProductBacklog }\end{array}$ & $06 / 07 / 2018$ & $\begin{array}{l}\text { - Análisis } \\
\text { Requerimientos } \\
\text { ProductBacklog }\end{array}$ \\
\hline & $\begin{array}{l}\text { - Entrega del Sprint } \\
\text { Backlog }\end{array}$ & 06/07/2018 & - Sprint Backlog \\
\hline $\begin{array}{l}\text { Análisis } \\
\text { Diseño }\end{array}$ & $\begin{array}{l}\text { - Entrega del Análisis y } \\
\text { Diseño del Producto o } \\
\text { Historia de Usuario }\end{array}$ & $11 / 07 / 2018$ & $\begin{array}{llll}\text { - Análisis y } & \text { Diseño } & \text { del } \\
\text { Producto } & \text { o } & \text { Historia } & \text { de } \\
\text { Usuario } & & & \\
& & \end{array}$ \\
\hline \multirow[t]{2}{*}{$\begin{array}{l}\text { Programación e } \\
\text { Integración }\end{array}$} & $\begin{array}{l}\text { - Actualización del } \\
\text { Sprint Backlog }\end{array}$ & $23 / 07 / 2018$ & - Sprint Backlog \\
\hline & $\begin{array}{l}\text { - Actualización del } \\
\text { ProductBacklog }\end{array}$ & $23 / 07 / 2018$ & - ProductBacklog \\
\hline
\end{tabular}




\section{(1)}

\begin{tabular}{|c|c|c|c|}
\hline & $\begin{array}{l}\text { - Programación de los } \\
\text { módulos del software }\end{array}$ & $23 / 07 / 2018$ & - Sistema JIRA \\
\hline & $\begin{array}{l}\text { - Pruebas de los } \\
\text { módulos del software }\end{array}$ & $15 / 08 / 2018$ & $\begin{array}{l}\text { - Sistema JIRA Software } \\
\text { probado }\end{array}$ \\
\hline & $\begin{array}{l}\text { - Entrega del Informe } \\
\text { general de pruebas }\end{array}$ & $23 / 08 / 2018$ & $\begin{array}{l}\text { - Informe general de } \\
\text { Pruebas }\end{array}$ \\
\hline & $\begin{array}{l}\text { - Entrega del Manual } \\
\text { de Instalación }\end{array}$ & $23 / 07 / 2018$ & - Manual de Instalación \\
\hline \multirow[t]{3}{*}{ Certificación } & $\begin{array}{l}\text { - Entrega de la solución } \\
\text { probada }\end{array}$ & $26 / 07 / 2018$ & $\begin{array}{l}\text { - Sistema JIRA Software } \\
\text { probado }\end{array}$ \\
\hline & $\begin{array}{l}\text { - Certificación de la } \\
\text { solución }\end{array}$ & $22 / 08 / 2018$ & $\begin{array}{l}\text { - Sistema JIRA Software } \\
\text { certificado }\end{array}$ \\
\hline & $\begin{array}{l}\text { - Entrega del Informe } \\
\text { final de certificación }\end{array}$ & $23 / 08 / 2018$ & $\begin{array}{l}\text { - Informe final de } \\
\text { certificación }\end{array}$ \\
\hline \multirow[t]{2}{*}{ Implementación } & $\begin{array}{l}\text { - } \begin{array}{l}\text { Entrega del } \\
\text { documento } \\
\text { Implementación }\end{array} \\
\end{array}$ & $27 / 08 / 2018$ & $\begin{array}{l}\text { - Documento } \\
\text { Implementación }\end{array}$ \\
\hline & $\begin{array}{l}\text { - Realización del pase a } \\
\text { soporte }\end{array}$ & $28 / 08 / 2018$ & $\begin{array}{l}\text { - Acta de aceptación del } \\
\text { pase a soporte }\end{array}$ \\
\hline \multirow[t]{2}{*}{ Cierre } & $\begin{array}{l}\text { - Entrega del acta de } \\
\text { conformidad del } \\
\text { cliente }\end{array}$ & $29 / 08 / 2018$ & $\begin{array}{l}\text { - Acta de conformidad del } \\
\text { cliente }\end{array}$ \\
\hline & $\begin{array}{l}\text { - Entrega del Acta de } \\
\text { conformidad ADC }\end{array}$ & 29/08/2018 & - Acta de conformidad ADC \\
\hline \multirow[t]{3}{*}{$\begin{array}{l}\text { Gestión de } \\
\text { Proyectos }\end{array}$} & $\begin{array}{l}\text { - Entregar matriz de } \\
\text { riesgos }\end{array}$ & $29 / 08 / 2018$ & - Matriz de riesgos \\
\hline & - Entrega de EDT & $24 / 08 / 2018$ & - EDT \\
\hline & $\begin{array}{l}\text { - Entrega } \\
\text { cronograma }\end{array}$ & $24 / 08 / 2018$ & - Cronograma \\
\hline
\end{tabular}

Nota: Se ha descrito las fases, hitos, fechas estimadas y entregables del proyecto. Elaboración propia. 


\section{(1)}

\section{Enfoques del trabajo}

En primer lugar se realizará el análisis de la situación actual aplicando arquitectura empresarial basada en el marco de trabajo TOGAF. Se identificará el AS IS y el TO $\mathrm{BE}$ de la empresa con la finalidad de detectar las brechas que se presentan en el proceso de Gestión de Proyectos.

La propuesta de arquitectura abarca los cuatro dominios de la AE: Negocio, Aplicaciones, Datos y Tecnológica. De acuerdo al Método de Desarrollo de la Arquitectura (ADM), establecida por TOGAF, el proyecto comprende desde la fase "Preliminar" hasta la fase "Oportunidades y Soluciones", considerando los siguientes entregables:

- Petición de Trabajo de Arquitectura

- Documento de Definición de Arquitectura

- Plan de Implementación de la Migración

Luego se identificará el proyecto de desarrollo en el cual se empleará el framework Scrum para poder implementar la solución propuesta.

Los proyectos identificados en la propuesta son:

\section{Proyecto de Mejora del procedimiento de Gestión de Proyectos}

En la fase de iniciación se solicitará toda la documentación relacionada al proceso de gestión de proyectos para su actualización, dichos entregables son: Instrucción de elaboración de la plantilla de riesgos, Instrucción de actualización de cronograma, Instrucción de criterios básicos para generar una solicitud de cambio, Procedimiento de Gestión de Proyecto, Procedimiento de Gestión de Requerimientos, Procedimiento de Gestión de Riesgos. Luego de ello en la fase de ejecución, se procederá actualizar todos los entregables con respecto al nuevo sistema a implementar. Posteriormente en la fase de verificación, luego de terminar la actualización de los cambios se deberá validar por el Analista de Calidad, en caso existan observaciones se tendrá que solucionar, caso contrario se deberán ingresar todos los entregables actualizados. Luego de ello en la etapa de cierre, se deberán ingresar al repositorio los documentos de proceso de gestión de proyectos actualizados. Por ultimo en la etapa de capacitación, se debe preparar una capacitación 


\section{(1)}

sobre todos los cambios del proceso de gestión de proyecto a todo el personal de operaciones.

\section{Proyecto de Implementación Software JIRA}

En la iniciación del proyecto se tendrá que preparar el cronograma con los tiempos estimados de inicio y finalización de cada tarea. Por ser un proyecto de aproximadamente 2 meses se realizará la entrega de ciertos documentos de gestión que pide la empresa al tener el certificado CMMI (nivel 3) e ISO 9001: 2008. Dentro de esta etapa se entregará la lista de personalización. Como artefactos que se presentarán bajo el marco de trabajo Scrum serán el Product Backlog y el Sprint Backlog. Tener en consideración que el grupo estará conformado de 9 personas, donde 4 de estos serán externos. Se tendrá el equipo de desarrollo liderado por un scrum master (todos externos), el equipo de calidad liderado por otro scrum master (internos) y por último el product owner (interno). Todo el grupo deberá realizar reuniones de manera obligatoria para poder ejecutar de manera correcta y óptima la solución. Para la etapa de análisis y diseño se realizarán las historias de usuarios identificadas y priorizadas por el producto owner en el Product Backlog.

En la fase de programación e integración, al ser un proyecto Scrum, se tendrán Sprints donde los artefactos Product Backlog y Sprint Backlog serán afinados en el transcurso del proyecto según sea conveniente. Se tendrán las actividades de programación y se dará como resultado el incremente del software JIRA. De la misma manera se presentarán las pruebas, el informe general y el manual de instalación del producto.

Para la certificación final, se hará entrega de la solución probada y certificada dando como resultado el informe final de certificación.

Posteriormente, en la implementación se hará entrega del documento de implementación y el acta de aceptación del pase a soporte a los servidores preparados por el área de soporte. Finalmente, para el cierre se hará entrega del acta de conformidad del cliente y el acta de conformidad ADC.

Como etapa transversal se tiene la Gestión de Proyectos tal y como se muestra en el mapa de procesos, donde se tendrán que actualizar de manera constante la matriz de riesgos, el EDT y el cronograma si fueran necesarios. 


\section{(4)}

\section{Riesgos y Mitigación}

Tabla 113

Cuadro de Riesgos y Mitigación

\begin{tabular}{|c|c|c|c|c|}
\hline$\#$ & Riesgo & Probabilidad & Impacto & Estrategia de respuesta al riesgo \\
\hline 1 & Mes del Mundial & 3 & Alto & $\begin{array}{l}\text { Los recursos del equipo podrán canjear sus } \\
\text { horas de trabajo por ver los partidos del } \\
\text { mundial en el comedor de la empresa, pero } \\
\text { tendrán que planificar su recuperación en la } \\
\text { semana previa coordinación con su líder } \\
\text { para que no se vea afectado el entregable. }\end{array}$ \\
\hline 2 & $\begin{array}{l}\text { Incremento en la rotación } \\
\text { de personal }\end{array}$ & 3 & Medio & $\begin{array}{l}\text { Fidelizar a los trabajadores realizando } \\
\text { actividades integradoras por lo menos una } \\
\text { vez al mes. Mejorar la comunicación con el } \\
\text { personal a través de reuniones personal-jefe } \\
\text { directo para identificar las expectativas y } \\
\text { problemática de cada área. }\end{array}$ \\
\hline 3 & Salud del Trabajador & 2 & Medio & $\begin{array}{l}\text { Concientizar al equipo sobre el clima ya que } \\
\text { para este año se ha detectado temperaturas } \\
\text { bajas y constantes lluvias. }\end{array}$ \\
\hline 4 & 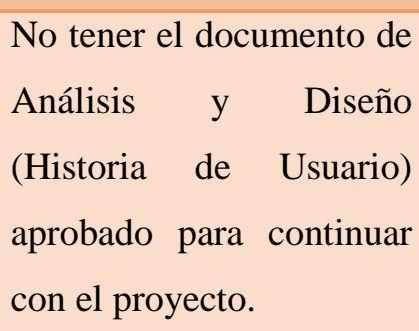 & 2 & Alto & $\begin{array}{l}\text { Evaluar migración a metodologías agiles. } \\
\text { Concientizar la importancia de tener el } \\
\text { documento de Análisis y Diseño aprobado. }\end{array}$ \\
\hline 5 & $\begin{array}{ll}\text { No actualizar la } \\
\text { documentación del } \\
\text { proceso de Gestión de } \\
\text { Proyectos. }\end{array}$ & 2 & Medio & $\begin{array}{l}\text { Validar semanalmente mediante el área de } \\
\text { control la actualización del proceso. }\end{array}$ \\
\hline 6 & $\begin{array}{l}\text { No tener los servidores } \\
\text { disponibles. }\end{array}$ & 2 & Alto & $\begin{array}{l}\text { Validar semanalmente con el área de } \\
\text { soporte y logística sobre dichas } \\
\text { adquisiciones. }\end{array}$ \\
\hline
\end{tabular}

Nota: Se ha descrito la probabilidad, impacto y estrategia de respuesta de los riesgos. Adaptado de "Matriz de Riesgos y Oportunidades”, por El Objeto de Estudio, 2017. 


\section{(1)}

\section{ESTUDIO DE FACTIBILIDAD}

En esta sección se evaluará los ingresos y egresos proyectados que genera la inversión para la implementación de los proyectos. Para desarrollar el estudio de factibilidad se han planteado varios supuestos ya que no se tenía acceso a la información de la organización.

Se ha revisado información sobre el tiempo promedio que puede tener un proyecto interno y la solución a proponer está dentro de los parámetros establecidos y por ende es viable la aplicación de este. Por otro lado, los recursos con los que cuenta la empresa cubren las necesidades que demanda el proyecto al tener el perfil adecuado para cubrir cada rol que se propone.

De igual forma, se realizó un cuadro de ingresos (ahorros en montos de las horas invertidas de los recursos que usan las herramientas de gestión de proyectos), en el cual se detalla:

- Los recursos involucrados

- Costo total de las horas mensuales

○ Cantidad de recursos existentes

- El monto total ahorrado anual por perfil, el cual es el costo de cada recurso multiplicado por el tiempo que se va a ahorrar con el JIRA (estimado), por la cantidad de recursos que tiene la empresa y por el periodo de un año.

\begin{tabular}{|c|c|c|c|c|c|}
\hline \begin{tabular}{|l} 
RECURSO \\
INVOLUCRADO \\
\end{tabular} & $\begin{array}{l}\text { CANTIDAD } \\
\text { RECURSO } \\
\end{array}$ & \begin{tabular}{|l|} 
COSTO POR \\
HORA $(\mathrm{S} /)$. \\
\end{tabular} & \begin{tabular}{|l} 
TIEMPO \\
MENSUAL \\
\end{tabular} & $\begin{array}{l}\text { TOTAL AHORRADO } \\
\text { MENSUAL (S/.) } \\
\end{array}$ & $\begin{array}{l}\text { TOTAL AHORRADO } \\
\text { ANUAL (S/.) } \\
\end{array}$ \\
\hline Lider Técnico & 3 & 37.5 & 14 & $1,575.00$ & $18,900.00$ \\
\hline Analista de Sistemas & 3 & 34.4 & 8 & 825.60 & $9,907.20$ \\
\hline Analista Programador & 15 & 31.25 & 6 & $2,812.50$ & $33,750.00$ \\
\hline Programador & 25 & 26.8 & 6 & $4,020.00$ & $48,240.00$ \\
\hline Analista de Procesos & 3 & 34.4 & 8 & 825.60 & $9,907.20$ \\
\hline Analista de Calidad & 8 & 34.4 & 8 & $2,201.60$ & $26,419.20$ \\
\hline Analista de Pruebas & 8 & 31.25 & 8 & $2,000.00$ & $24,000.00$ \\
\hline Arquitecto de Sistemas & 2 & 34.4 & 6 & 412.80 & $4,953.60$ \\
\hline Analista de Base de Datos & 2 & 34.4 & 6 & 412.80 & $4,953.60$ \\
\hline
\end{tabular}

Figura 38. Cuadro del total ahorrado por recurso. Elaboración Propia 


\section{(1)}

Se muestra los ingresos y egresos proyectados para la propuesta.

\begin{tabular}{|c|c|c|c|c|}
\hline PERIODO ANUAL & 2018 & 2019 & 2020 & 2021 \\
\hline \multicolumn{5}{|l|}{ INGRESOS } \\
\hline Monto ahorrado por horas de lideres técnicos & 0.00 & $18,900.00$ & $18,900.00$ & $18,900.00$ \\
\hline Monto ahorrado por horas de analistas de sistemas & 0.00 & $9,907.20$ & $9,907.20$ & $9,907.20$ \\
\hline Monto ahorrado por horas de analistas programadores & 0.00 & $33,750.00$ & $33,750.00$ & $33,750.00$ \\
\hline Monto ahorrado por horas de programadores & 0.00 & $48,240.00$ & $48,240.00$ & $48,240.00$ \\
\hline Monto ahorrado por horas de analistas de procesos & 0.00 & $9,907.20$ & $9,907.20$ & $9,907.20$ \\
\hline Monto ahorrado por horas de analistas de calidad & 0.00 & $26,419.20$ & $26,419.20$ & $26,419.20$ \\
\hline Monto ahorrado por horas de analistas de pruebas & 0.00 & $24,000.00$ & $24,000.00$ & $24,000.00$ \\
\hline Monto ahorrado por horas de arquitectos de sistemas & 0.00 & $4,953.60$ & $4,953.60$ & $4,953.60$ \\
\hline Monto ahorrado por horas de analistas de base de datos & 0.00 & $4,953.60$ & $4,953.60$ & $4,953.60$ \\
\hline TOTAL INGRESOS & 0.00 & $181,030.80$ & $181,030.80$ & $181,030.80$ \\
\hline \multicolumn{5}{|l|}{ EGRESOS } \\
\hline Gastos en servidores y licencias & $120,000.00$ & $21,780.00$ & $21,780.00$ & $21,780.00$ \\
\hline Gastos por personal de arquitectura empresarial & $28,500.00$ & 0.00 & 0.00 & 0.00 \\
\hline Gastos por personal de implementación & $47,008.00$ & 0.00 & 0.00 & 0.00 \\
\hline Gastos de personalización & $15,000.00$ & 0.00 & 0.00 & 0.00 \\
\hline TOTAL EGRESOS & $210,508.00$ & $21,780.00$ & $21,780.00$ & $21,780.00$ \\
\hline FLUJO EFECTIVO & $-210,508.00$ & $159,250.80$ & $159,250.80$ & $159,250.80$ \\
\hline FLUJO ACUMULADO & $-210,508.00$ & $-51,257.20$ & $107,993.60$ & $267,244.40$ \\
\hline TASA DESCUENTO & 0.15 & & & \\
\hline VAN & $153,097.43$ & & & \\
\hline TIR & 0.56 & & & \\
\hline
\end{tabular}

Figura 39. Cuadro de Flujo de Inversión. Elaboración Propia

A continuación, se realiza el detalle de la información del Flujo de Inversión:

- Como periodo de evaluación se tiene 3 años a partir del 2019, el año 2018 es lo que se gastará.

- El total de ingresos es la suma total ahorrado de los recursos dando un total de S/.181,030.80.

○ El total de egresos durante el primer periodo es S/.210, 508.00, luego como gasto anual para los siguientes periodos, por temas de licencias para el software, es de S/. 21,780.00 


\section{(1)}

- El total de flujo de efectivo del primer periodo por la implementación de los proyectos es de S/. - 210,508.00. Luego los siguientes periodos se obtiene de la resta de total de ingresos menos total de egresos, dando un flujo de efectivo de S/.159,250.80.

- Como tasa de descuento se usó el 15\%

- Para hallar el VAN se utilizó la tasa de descuento de $15 \%$ y se acumularon los flujos de efectivo desde el año 2019 hasta el 2021 de S/.159,250.80, luego se sumará el total de flujo efectivo del primer periodo S/.-210,508.00 y finalmente da como resultado un valor de S/.153,097.43.

- Para hallar el TIR se utilizó el total de flujo efectivo del primer periodo de S/.210,508.00 y los flujos de efectivo de los siguientes 3 periodos de S/.159,250.80 dando como resultado un total de $56 \%$.

\section{$\underline{\text { Análisis Financiero }}$}

El estudio se ha realizado en base al ahorro en montos por horas de los recursos por rol que invertían un tiempo estimado de horas de manera semanal en los antiguos sistemas.

Se identificó que el VAN da un resultado positivo aplicando la tasa de descuento que se ofrece en el mercado (15\%), obteniendo un tiempo de retorno de inversión de 2 años.

El TIR calculado llega al 56\% anual, superando a la tasa de descuento que se ofrece en el mercado de $15 \%$ demostrando que la inversión en el proyecto es mejor.

Como conclusión se puede indicar que el proyecto es viable económicamente, aparte de ello, se podrá apreciar el ahorro de tiempo de los trabajadores al utilizar la nueva herramienta de Gestión de Proyectos y podrán emplear sus tiempos en actividades productivas. 


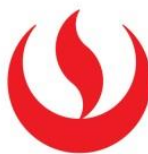

\section{BIBLIOGRAFÍA}

Alarcón A., Ullua P. \& Carretero S. (2014) Las Decisiones de Presupuesto de Capital: Criterios Financieros Fundamentales. . Repositorio Institucional de la Universidad de Ciego de Ávila, p.8. Recuperado de http://xn--caribea-9za.eumed.net/wpcontent/uploads/presupuestos.pdf [Consulta: 01 de junio de 2018].

Arango-Serna, M., Londoño-Salazar, J., \& Branch-Bedoya, J. (2015). Enfoque de arquitectura de solución, mecanismo para reducir la brecha entre la arquitectura empresarial y la implementación de soluciones tecnológicas. Revista de Ingenería Dyna, 82 (193), 117126.

Navarro, A., Fernández, J., \& Morales, J. (2013). Revisión de metodologías ágiles para el desarrollo de software. Prospectiva, 11 (2), 30 - 39.

ProyectoAgiles.org ("s.f."). Beneficios de Scrum. Recuperado de https://proyectosagiles.org/beneficios-de-scrum/ [Consulta: 29 de marzo de 2018].

The Open Group (1999 - 2018). Welcome to the TOGAF® Standard, Version 9.2, a standard of The Open Group. The United States: The Open Group. Recuperado de http://pubs.opengroup.org/architecture/togaf9-doc/arch/index.html [Consulta: 30 de abril de 2018].

Zachman J. (1987). A Framework For Information-Systems Architecture. IBM Systems Journal. 26 (3), 276-292 


\section{(1)}

\section{CONCLUSIONES}

- Se ha logrado puntualizar los fundamentos del trabajo en el marco teórico, para fortalecer los conocimientos sobre el objeto de estudio y efectuar un análisis más detallado de los problemas y necesidades del campo de acción.

- Se ha realizado una propuesta de Arquitectura Empresarial del proceso de Gestión de Proyectos, que consiste en dar un enfoque integral sobre las iniciativas y proyectos que una organización tiene para mejorar, transformar y adaptarse, pero no solo es exclusivo sobre diseño de software o modelamiento de proceso sino todo lo que abarque sus cuatro dominios.

- Se ha conseguido entender que Scrum funciona de una manera correcta dentro de un ámbito específico, caracterizado por equipos pequeños de desarrollo, con clientes que pueden tomar decisiones acerca de los requisitos lo cuales pueden variar con frecuencia (semanal o mensual) y porque es adaptable con la metodología que tiene la organización.

- Se ha logrado construir una propuesta que libera las mejores prácticas de cada uno de los frameworks analizados en el desarrollo del proyecto: TOGAF y Scrum, que soporte el proceso de Gestión de Proyecto el cual es un proceso clave definido en el análisis de Arquitectura Empresarial.

- Se ha podido identificar que la organización si bien se dedica a ofrecer soluciones transaccionales tecnológicas para dar un mejor soporte a los negocios de los clientes, no realiza un análisis de actualización de sistemas y herramientas internas. 


\section{(1)}

\section{RECOMENDACIONES}

- Concientizar a la alta gerencia de siempre tener claro la misión, visión, objetivos generales y estratégicos de la empresa apoyándose de un planeamiento estratégico para que así la empresa y sus colaboradores tengan una dirección clara hacia dónde quiere llegar la empresa.

- Si bien se ha realizado la propuesta del estudio usando el marco de trabajo TOGAF se recomienda evaluar otros criterios de los marcos de trabajo existentes para poder identificar cual otro se puede adaptar mejor a la empresa.

- Si bien se ha realizado la propuesta del estudio usando el marco de trabajo TOGAF se recomienda validar con otros métodos que se pueden complementar para contar con una arquitectura empresarial robusta.

- Crear un área de arquitectura empresarial basándose en los resultados y beneficios que se pueden lograr gracias al uso de una buena arquitectura empresarial y no teniéndolo como algo complementario que no se podrá explotar de la manera correcta. 


\section{(1)}

\section{GLOSARIO DE TÉRMINOS}

Gateway: Permite interconectar redes con protocolos y arquitecturas diferentes a todos los niveles de comunicación.

Job: Es un conjunto coherente de instrucciones para realizar un trabajo particular.

Off-host: Es una modalidad de trabajo cuando el computador no está disponible.

Teleacceso: Permite acercarnos no a tecnologías individuales sino a las percepciones sociales de la tecnología, que sustentan, finalmente, su posibilidad de éxito. 


\section{SIGLARIO}

AE: Arquitectura Empresarial

CSP: Control y Seguimiento de Proyectos

ISO: International Organization for Standardization

SIX/EFT Switch: Transferencia Electrónica de Fondos

SIX/TCL: Teleacceso de Clientes

TOGAF: The Open Group Architecture Framework

XP: Extreme Programming 


\section{(}

\section{BIBLIOGRAFÍA}

Adriana, M. (27 de enero de 2015). ¿Qué es Arquitectura Empresarial? Colombia Digital, p. 1. Recuperado de https://colombiadigital.net/actualidad/articulos-informativos/item/8123que-es-arquitectura-empresarial.html [Consulta: 29 de marzo de 2018].

Arango-Serna, M., Londoño-Salazar, J., \& Branch-Bedoya, J. (2015). Enfoque de arquitectura de solución, mecanismo para reducir la brecha entre la arquitectura empresarial y la implementación de soluciones tecnológicas. Revista de Ingenería Dyna, 82 (193), 117126.

Arango, M., Londoño, J., \& Zapata, J. (2010). Arquitectura Empresarial - Una Visión General. Revista ingenierías Universidad de Medellín, 9 (16), 101-111.

Atlassian (2001). La herramienta de desarrollo de software líder de los equipos agiles. Australia: Atlassian. Recuperado https://es.atlassian.com/software/jira [Consulta: 30 de marzo de 2018].

Avison, D., \& Fitzgerald, G. (1995). Information systems development: methodologies, techniques and tools. Maidenhead: McGraw-Hill.

Cabrera, J. (2018). Análisis FODA [Presentación para el curso Planeamiento Estratégico]. Lima: Universidad Peruana de Ciencias Aplicadas.

Canós, J., Penadés, C., \& Letelier, P. (Marzo 2012). Metodologías Ágiles en el Desarrollo de Software. Repositorio Institucional de la Universidad de Las Tunas, p.4. Recuperado de http://roa.ult.edu.cu/bitstream/123456789/476/1/TodoAgil.pdf [Consulta: 30 de marzo de 2018]. 


\section{(1)}

Chandra, V. (2015). Comparison between Various Software Development Methodologies. International Journal of Computer Applications, p. 3. Recuperado de https://pdfs.semanticscholar.org/e237/f9cb136f494c2bd0ce91525808c5c968b6b4.pdf [Consulta: 26 de mayo de 2018].

Craftware Consultores LTDA (2014). Caso de Éxito: Modelamiento fundacional para el ' ejercicio de la Arquitectura Empresarial. Chile: Craftware Consultores LTDA. Recuperado de http://craftware.net/wp-content/uploads/2016/06/Caso-de-Exito-Modelamientofundacional-para-el-ejercicio-de-la-Arquitectura-Empresarial.pdf [Consulta: 29 de marzo de 2018].

Evaluandosoftware.com (2016). Arquitectura empresarial ¿qué es y para qué sirve?. Recuperado de http://www.evaluandosoftware.com/arquitectura-empresarial/ [Consulta: 29 de marzo de 2018].

Goikolea, M. (2014). Éxitos y fracasos en proyectos Scrum: Spotify vs Healthcare IEBS, p. 1. Recuperado https://www.iebschool.com/blog/exitos-y-fracasos-en-proyectos-scrum-spotifyvs-healthcare-agile-scrum/ [Consulta: 30 de marzo de 2018].

Juan, P. (2015) Scrum Manager I: Las reglas de scrum Scrummanager.net, p. 19. Recuperado de http://www.scrummanager.net/files/scrum_I.pdf [Consulta: 30 de marzo de 2018].

Juan, V. Eventos Scrum: El Scrum Diario. Management Plaza, p.1. Recuperado de http://managementplaza.es/blog/el-scrum-diario/ [Consulta: 30 de marzo de 2018].

Kenneth, R. Scrum roles y responsabilidades Club de Tecnología, p.1. Recuperado de http://www.clubdetecnologia.net/blog/2015/scrum-roles-responsabilidades/ [Consulta: $30 \mathrm{de}$ marzo de 2018].

Navarro, A., Fernández, J., \& Morales, J. (2013). Revisión de metodologías ágiles para el desarrollo de software. Prospectiva, 11 (2), 30 - 39. 


\section{(4)}

The Open Group (1999 - 2018). Welcome to the TOGAF® Standard, Version 9.2, a standard of The Open Group. The United States: The Open Group. Recuperado de http://pubs.opengroup.org/architecture/togaf9-doc/arch/index.html [Consulta: 30 de abril de 2018].

Objeto de Estudio (2017). Manual de Calidad. Lima: Objeto de Estudio.

Objeto de Estudio (2017). Manual de funciones y perfiles de puestos. Lima: Objeto de Estudio.

ProyectoAgiles.org ("s.f."). Beneficios de Scrum. Recuperado de https://proyectosagiles.org/beneficios-de-scrum/ [Consulta: 29 de marzo de 2018].

ProyectoAgiles.org (“s.f.”). Estimación y planificación ágil - Resultados del quinto encuentro ágil en Barcelona. Recuperado de https://proyectosagiles.org/2009/07/01/estimacion-planificacion-agil-quinto-encuentro-agilbarcelona/\#planning-poker [Consulta: 29 de marzo de 2018].

ProyectoAgiles.org ("s.f."). Lista de tareas de la iteración (Sprint Backlog). Recuperado de https://proyectosagiles.org/lista-tareas-iteracion-sprint-backlog/\#tablero-tareas-taskboard [Consulta: 29 de marzo de 2018].

ProyectoAgiles.org (“s.f.”). Planificación ágil vs planificación tradicional. Recuperado de https://proyectosagiles.org/2010/12/15/planificacion-agil-vs-planificacion-tradicional/ [Consulta: 29 de marzo de 2018].

Schwaber, K., \& Sutherland, J. (2016). La Guía Definitiva de Scrum: Las Reglas del Juego. Recuperado de http://www.scrumguides.org/docs/scrumguide/v2016/2016-Scrum-GuideSpanish.pdf\#zoom=100 [Consulta: 29 de marzo de 2018]. 
Siliceo, A. (2001). Capacitación y Administración de personal. En J. Diez \& J. Abreu (Ed.), Impacto de la capacitación interna en la productividad y estandarización de procesos productivos: un estudio de caso. p.26. México: Editorial Limusa.

Subauste, D. (2018). Cynefin [Presentación para el curso Metodologías Ágiles]. Lima: Universidad Peruana de Ciencias Aplicadas.

Zachman J. (1987). A Framework For Information-Systems Architecture. IBM Systems Journal. 26 (3), 276-292 


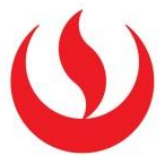

\section{ANEXOS}

\section{Anexo 1}

\section{novatronic}

\section{APROBACIÓN DE TEMA DE TESIS}

Yo, Patricia Díaz Chávez

Código de alumno: u201200200

Yo, Raal Rojas Arroyo

Código de alumno: u201114499

De la carrera de Ingeniería de Sistemas EPE, autor(a/es) de la Tesis titulada:

Propuesta de una arquitectura empresarial para una consultora de software

DECLARO QUE:

El tema de tesis es auténtico, siendo resultado de un trabajo personal, que no se ha copiado. y que menciona de forma clara y exacta su origen 0 autor, tanto en el cuerpo del texto, ilustraciones, cuadros, tablas u otros que tengan derechos de autor.

La Empresa NOVATRONIC SAC tiene conocimiento de la información utilizada en la elaboración del documento y autoriza que concluido el documento tesis, éste quede publicado en la web de la universidad, en la modalidad:

[ ] pública, lo cual significa que cualquier persona natural o jurídica puede acceder al contenido

[ $x$ ] privada, lo cual significa que durante los tres primeros años, toda persona natural o juridica interesada en acceder al contenido, deberá contar con autorización de la empresa. Pasados los tres años el contenido de la tesis pasa a modo público.

En este sentido, soy (somos) consciente(s) de que el hecho de no respetar los derechos de autor, y/o no tener el consentimiento de la empresa objeto de estudio, son objeto de sanciones universitarias y/o legales.

Lima, 14 de Junio de 2018

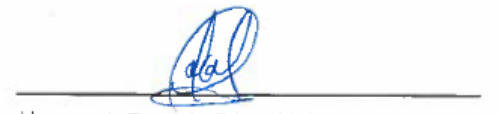

Alumno 1: Patricia Diaz Chávez

Código de alumno. u201200200

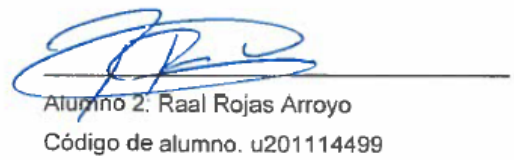

Código de alumno. u201114499

\section{NOVALRONIC SAC.}

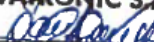

SARTAARANGUENAA

Sarita Arangüena Alcántar

Jefe de Control

Novatronic SAC

Av. José Gálvez Barrenechea 1094, Lima 27 - PERU Telf. (511) 415-2400

página web: www.novatronic.com e-mail: info@novatronic.com

Figura 40. Carta de Aprobación Objeto de Estudio. Adaptado de "Carta de aprobación de tema de tesis", por UPC, 2018. 


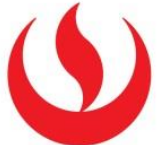

\section{Anexo 2}

\begin{tabular}{|c|c|c|c|c|c|c|c|c|c|}
\hline \multirow[b]{2}{*}{ Periodo } & \multicolumn{4}{|c|}{ Situación Actual } & \multicolumn{4}{|c|}{ Situación Deseada } & \multirow[b]{2}{*}{ Reducción } \\
\hline & $\begin{array}{l}\text { Actualización de } \\
\text { Cronogramas } \\
\text { (tiempo horas) }\end{array}$ & \begin{tabular}{|l|} 
Procesamiento \\
de cronogramas \\
(tiempo horas)
\end{tabular} & $\begin{array}{l}\text { Total de Horas } \\
\text { Semanales }\end{array}$ & $\begin{array}{l}\% \text { semanal de } \\
\text { Horas }\end{array}$ & \begin{tabular}{|l} 
Actualización de \\
Cronogramas \\
(tiempo horas)
\end{tabular} & \begin{tabular}{|l|} 
Procesamiento de \\
cronogramas \\
(tiempo horas)
\end{tabular} & $\begin{array}{l}\text { Total de Horas } \\
\text { Semanales }\end{array}$ & $\begin{array}{l}\% \text { semanal } \\
\text { de Horas }\end{array}$ & \\
\hline $07 / 06 / 2018$ & 6 & 2 & 8 & $20 \%$ & & & & & $0 \%$ \\
\hline $14 / 06 / 2018$ & 6 & 1.5 & 7.5 & $19 \%$ & & & & & $0 \%$ \\
\hline $21 / 06 / 2018$ & 5 & 2 & 7 & $18 \%$ & & & & & $0 \%$ \\
\hline $28 / 06 / 2018$ & 6 & 2 & 8 & $20 \%$ & & & & & $0 \%$ \\
\hline $05 / 07 / 2018$ & 6 & 2 & 8 & $20 \%$ & & & & & $0 \%$ \\
\hline $12 / 07 / 2018$ & 6 & 1.5 & 7.5 & $19 \%$ & & & & & $0 \%$ \\
\hline $19 / 07 / 2018$ & 5 & 2 & 7 & $18 \%$ & & & & & $0 \%$ \\
\hline $26 / 07 / 2018$ & 6 & 2 & 8 & $20 \%$ & & & & & $0 \%$ \\
\hline $02 / 08 / 2018$ & 6 & 2.5 & 8.5 & $21 \%$ & & & & & $0 \%$ \\
\hline $09 / 08 / 2018$ & 5.5 & 2 & 7.5 & $19 \%$ & & & & & $0 \%$ \\
\hline $16 / 08 / 2018$ & 6 & 2 & 8 & $20 \%$ & & & & & $0 \%$ \\
\hline $23 / 08 / 2018$ & 6.5 & 1.5 & 8 & $20 \%$ & & & & & $0 \%$ \\
\hline $30 / 08 / 2018$ & 6 & 2 & 8 & $20 \%$ & & & & & $0 \%$ \\
\hline $06 / 09 / 2018$ & 6 & 2 & 8 & $20 \%$ & 4 & 0.5 & 4.5 & $11 \%$ & $9 \%$ \\
\hline $13 / 09 / 2018$ & 6 & 2 & 8 & $20 \%$ & 4 & 0.5 & 4.5 & $11 \%$ & $9 \%$ \\
\hline $20 / 09 / 2018$ & 6 & 2 & 8 & $20 \%$ & 4 & 0.5 & 4.5 & $11 \%$ & $9 \%$ \\
\hline $27 / 09 / 2018$ & 6 & 2 & 8 & $20 \%$ & 4 & 0.5 & 4.5 & $11 \%$ & $9 \%$ \\
\hline
\end{tabular}

Figura 41. Reducción de la actualización y procesamiento de cronogramas. Elaboración propia.

\section{Anexo 3}

\begin{tabular}{|c|c|c|c|c|c|c|}
\hline \multirow[b]{2}{*}{ Periodo } & \multicolumn{3}{|c|}{ Situación Actual } & \multicolumn{3}{|c|}{ Situación Deseada } \\
\hline & $\begin{array}{l}\text { No } \\
\text { conformidades a } \\
\text { proyectos }\end{array}$ & $\begin{array}{l}\text { No conformidades } \\
\text { al proceso }\end{array}$ & $\begin{array}{l}\text { Existe no } \\
\text { conformidad }\end{array}$ & $\begin{array}{l}\text { No } \\
\text { conformidades a } \\
\text { proyectos }\end{array}$ & $\begin{array}{l}\text { No conformidades } \\
\text { al proceso }\end{array}$ & \begin{tabular}{|l} 
Existe no \\
conformidad
\end{tabular} \\
\hline $12 / 09 / 2016$ & 0 & 1 & Si & & & \\
\hline $12 / 12 / 2016$ & 0 & 1 & $\mathrm{Si}$ & & & \\
\hline $13 / 03 / 2017$ & 0 & 1 & $\mathrm{Si}$ & & & \\
\hline $12 / 06 / 2017$ & 1 & $\underline{0}$ & $\mathrm{Si}$ & & & \\
\hline $11 / 09 / 2017$ & 0 & 1 & Si & & & \\
\hline $11 / 12 / 2017$ & 1 & 0 & Si & & & \\
\hline $12 / 03 / 2018$ & 0 & 1 & Si & & & \\
\hline $11 / 06 / 2018$ & 0 & 1 & $\mathrm{Si}$ & & & \\
\hline 10/09/2018 & 0 & 1 & $\mathrm{Si}$ & & & \\
\hline $10 / 12 / 2018$ & 0 & 1 & Si & 0 & 0 & No \\
\hline $11 / 03 / 2019$ & 0 & 1 & $\mathrm{Si}$ & 0 & 0 & No \\
\hline $10 / 06 / 2019$ & 0 & 1 & Si & $\underline{0}$ & 0 & No \\
\hline 09/09/2019 & 0 & 1 & $\mathrm{Si}$ & 0 & 0 & No \\
\hline
\end{tabular}

Figura 42. Reducción de no conformidades. Elaboración propia. 